Technische Universität München

Lehrstuhl für Metallbau

\title{
Design of Composite Slab Systems in Case of Fire Using Simplified Finite Element Analyses
}

\author{
Martin Stadler
}

Vollständiger Abdruck der von der Fakultät für Bauingenieur- und Vermessungswesen der Technischen Universität München zur Erlangung des akademischen Grades eines

Doktor-Ingenieurs

genehmigten Dissertation.

\author{
Vorsitzender: $\quad$ Univ.-Prof. Dr.-Ing. Oliver Fischer \\ Prüfer der Dissertation: \\ 1. \\ Univ.-Prof. Dr.-Ing. Martin Mensinger \\ 2. \\ Univ.-Prof. Dr.-Ing. Peter Schaumann, \\ Gottfried Wilhelm Leibniz Universität Hannover \\ 3. \\ Prof. Ian Burgess Ph.D., University of Sheffield / UK
}

Die Dissertation wurde am 29.02.2012 bei der Technischen Universität München eingereicht und durch die Fakultät für Bauingenieur- und Vermessungswesen am 18.06.2012 angenommen. 



\section{Abstract}

A new method is presented in this thesis for designing both full concrete and composite slab systems with partially unprotected secondary steel beams in case of fire taking into account tensile membrane action. Simplified finite element analyses are used for determining the internal forces in the structure. Crosssection design procedures can be applied with these internal forces to calculate the required reinforcement amount like under ambient temperature. The finite element model is simplified by replacing the thermal analysis with a substitute thermal loading. Non-linear material behaviour is taken into account by a reduced stiffness of the model which allows an efficient linear elastic calculation. The presented method therefore enables a simple and efficient design of slab systems in case of fire.

\section{Zusammenfassung}

In dieser Arbeit wird eine neue Methode zur Bemessung von Vollbeton- und Verbunddeckensystemen mit teilweise ungeschützten Stahl-Nebenträgern im Brandfall unter Berücksichtigung von Membrantragwirkung vorgestellt. Hierbei werden vereinfachte Finite Elemente Simulationen zur Schnittgrößenermittlung verwendet. Mit diesen Schnittgrößen können Querschnittsnachweise zur Ermittlung der erforderlichen Bewehrungsmengen wie unter Normaltemperatur geführt werden. Zur Vereinfachung des FE-Modells wird die thermische Analyse durch eine Temperaturbelastung ersetzt. Nichtlineares Materialverhalten wird durch eine vorab ermittelte Steifigkeitsreduzierung berücksichtigt was eine effiziente linear elastische Berechnung erlaubt. Das vorgestellte Verfahren ermöglicht somit eine einfache und effiziente Bemessung von Deckensystemen im Brandfall. 


\section{Acknowledgement}

First of all my special gratitude goes to Prof. Dr.-Ing. Dipl. Wirt.-Ing. (NDS) Martin Mensinger for giving me the opportunity to write this thesis at the Chair for Metal Structures (Lehrstuhl für Metallbau) at the Technische Universität München (Technical University of Munich). Besides his support and encouragement, I also thank him for the friendly and familiar atmosphere at the chair.

Furthermore, I would like to address my thanks to Prof. Dr.-Ing. Peter Schaumann for his interest and examining my work. I also want to thank Prof. Dr.-Ing. Dipl. Wirt.-Ing. Oliver Fischer for chairing the jury.

In the course of my research, I spent four months at the University of Sheffield at the department of civil and structural engineering under the supervision of Prof. Ian Burgess. I want to thank him for his kind hospitality, the stimulating discussions and that he agreed to examine my thesis.

I want to thank all colleagues at the Chair for Metal Structures for their friendly cooperation and the pleasant time that I had when I was working with them. My special thanks go to Peter Kraus for supporting me by preparing my fire tests.

Many thanks are addressed to Lucy Johnson for proofreading my thesis.

Finally, I want to thank my wife Monika for encouraging me to write this thesis and for her mental and financial support that helped me to finish this work.

Munich, August 2012

Martin Stadler 


\section{Contents}

1 Introduction $\quad 1$

1.1 Problem specification . . . . . . . . . . . . . . . . . . . . 1

1.2 Objective and layout of the thesis . . . . . . . . . . . . . . . . . . . 2

2 State of research $\quad 4$

2.1 Research projects . . . . . . . . . . . . . . . . . . . . 4

2.2 Advanced calculation models . . . . . . . . . . . . . . . . . . . . . 6

2.2.1 General numerical simulations . . . . . . . . . . . . . . . . 6

2.2.2 Specialised software .......................... 8

2.3 Simple calculation models . . . . . . . . . . . . . . . . . . . . 9

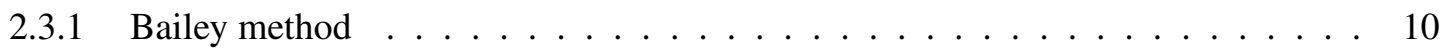

2.3.2 DASt design tables and construction details . . . . . . . . . . . . . . 12

3 Experiments 13

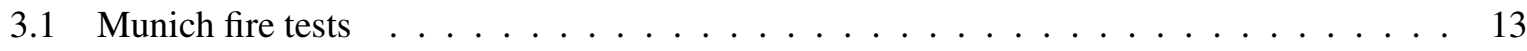

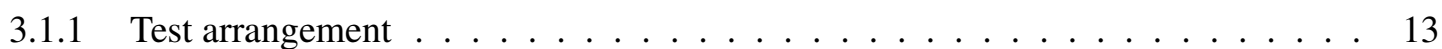

3.1 .2 Results test $1 \ldots \ldots \ldots \ldots$

3.1 .3 Results test 2 ...................... 22

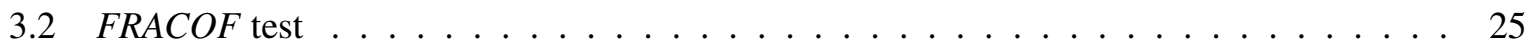

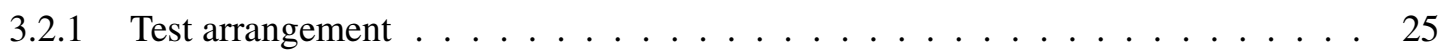

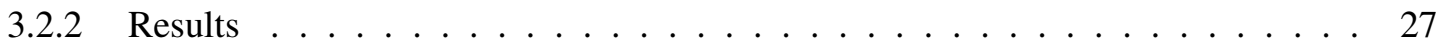

4 Thermal loading $\quad 30$

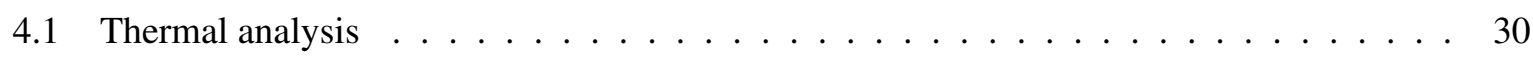

4.1.1 Full concrete slabs ...................... 31

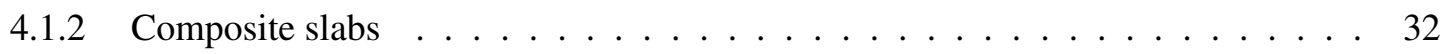

4.2 Substitute thermal loading on slabs . . . . . . . . . . . . . . . . . . . 37

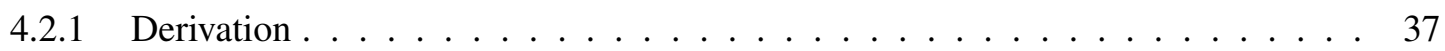

4.2.2 Full concrete slabs ... . . . . . . . . . . . . . . . . 40

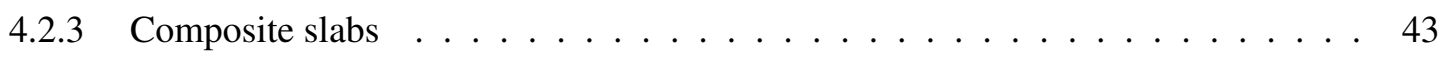

4.2 .4 Validation ........................ 48

4.3 Substitute thermal loading on beams . . . . . . . . . . . . . . . . . . . 49

5 Mechanical analysis $\quad 53$

5.1 Material non-linearities . . . . . . . . . . . . . . . . . 53

5.1 .1 Finite element formulations .................. 53

5.1.2 Analytical models for ambient temperature . . . . . . . . . . . . . 55 


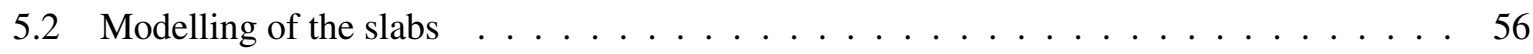

5.2 .1 Geometry and elements . . . . . . . . . . . . . . . . 56

5.2 .2 Influence of stiffness . . . . . . . . . . . . . . . . . . 57

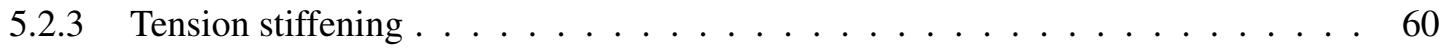

5.2 .4 Stiffness at the ultimate limit state . . . . . . . . . . . . . . . . . . 62

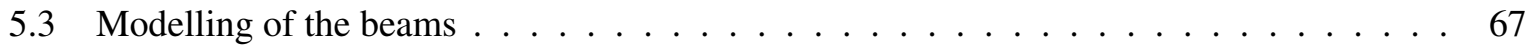

6 Design $\quad 73$

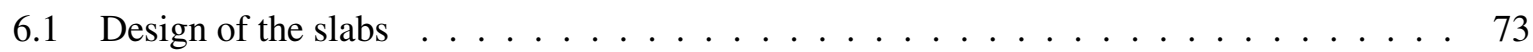

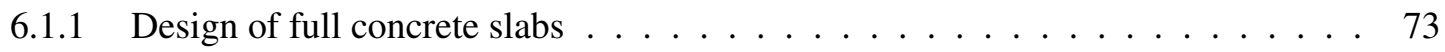

6.1 .2 Design of composite slabs . . . . . . . . . . . . . . 76

6.2 Design of the beams . . . . . . . . . . . . . . . . . . . 79

7 Validation on fire tests $\quad 85$

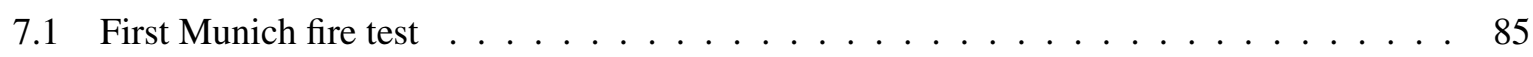

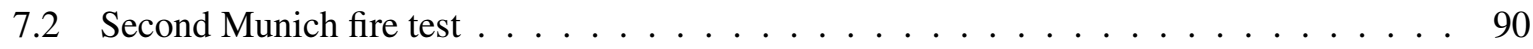

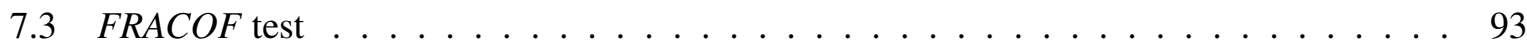

8 Worked example $\quad 97$

8.1 Modelling and results . . . . . . . . . . . . . . . . . . . . . . . 98

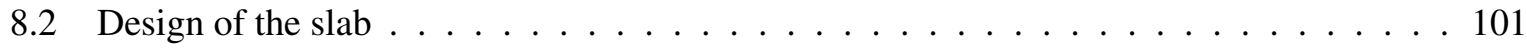

8.3 Design of the beams . . . . . . . . . . . . . . . . . . . 103

9 Summary and recommendations $\quad 105$

A Worked example for the substitute thermal loading of a full concrete slab $\quad 107$

$\begin{array}{ll}\text { B Input files } & 108\end{array}$

B.1 Example 4-2: Short beam . . . . . . . . . . . . . . . . . . . . . . . . . 108

B.2 Example 4-3: Composite beam with shell elements . . . . . . . . . . . . 111

B.3 Example 5-1: Influence of slab stiffness . . . . . . . . . . . . . . . . . . . 113

B.4 Example 5-2: Composite beam with beam elements . . . . . . . . . . . . . . . 116

B.5 Example 6-1: Load bearing characteristics of composite beam . . . . . . . . . . . 118

B.6 Validation on first Munich test . . . . . . . . . . . . . . . . . . 120

B.7 Validation on second Munich test . . . . . . . . . . . . . . . . . . . 125

B.8 Validation on FRACOF test . . . . . . . . . . . . . . . . . . . . . . 131

B.9 Worked example . . . . . . . . . . . . . . . . . . . . . . . . 134 


\section{List of Figures}

1.1 Tensile membrane action of a single slab panel . . . . . . . . . . . . . . . 1

1.2 Positioning of the research results . . . . . . . . . . . . . . . . . 2

2.1 Gaping crack nearby the intermediate beam in first Munich test . . . . . . . . . 5

2.2 Mohr-Coulomb failure criterion [55] . . . . . . . . . . . . . . . . . . 7

2.3 Failure mode (left) and forces along yield lines (right) according to [5] . . . . . . . . 10

3.1 Plan view of test 1 (above) and 2 (below) according to [42] . . . . . . . . . . . . 14

3.2 Slab cross-section of first (left) and second (right) Munich test . . . . . . . . . . . . 15

3.3 Section A-A of Munich fire tests according to [42] . . . . . . . . . . . . . . . 16

3.4 Mean gas temperature of first Munich test according to [42] . . . . . . . . . . . . . 17

3.5 Temperatures in slab of first Munich test according to [42] . . . . . . . . . . . . 17

3.6 Temperatures in protected edge beam and unprotected secondary beam of first Munich test according to [42] . . . . . . . . . . . . . . . . . . . . . . 18

3.7 Temperatures in protected intermediate beam of first Munich test according to [42] . . . 19

3.8 Vertical displacements of first Munich test according to [42] . . . . . . . . . . . . . 19

3.9 Crack pattern of first Munich test according to [42] . . . . . . . . . . . . . . . . 20

3.10 View from below at gaping crack at first Munich test according to [42] . . . . . . . . 20

3.11 Section with cracks of first Munich test according to [42] . . . . . . . . . . . . . . 21

3.12 Mean gas temperature of second Munich test according to [42] . . . . . . . . . . . 22

3.13 Temperatures in slab, measuring point D6, of second Munich test according to [42] . . 23

3.14 Temperatures in beams of second Munich test according to [42] . . . . . . . . . . 23

3.15 Vertical displacements of second Munich test according to [42] . . . . . . . . . . . . . . 24

3.16 Horizontal displacements of second Munich test according to [42] . . . . . . . . . . . . 24

3.17 Possible crack location, steel sheeting parallel to intermediate beam . . . . . . . . . 25

3.18 Plan view of FRACOF test on the basis of [49] . . . . . . . . . . . . . . . . . . . 26

3.19 Slab cross-section of FRACOF test . . . . . . . . . . . . . . . . . . . . . . 26

3.20 Temperatures in slab of FRACOF test according to [49] . . . . . . . . . . . . . . . 27

3.21 Temperatures in protected edge beam of FRACOF test according to [49] . . . . . . . . . 28

3.22 Vertical deformations of FRACOF test according to [49] . . . . . . . . . . . . . . . . 28

4.1 Comparison of the temperature distributions from Eurocode data with FEA . . . . . . 31

4.2 Comparison of temperatures from first Munich test with FEA . . . . . . . . . . . . . . 32

4.3 Composite slab cross-sections with re-entrant (left) and open (right) trough profile steel

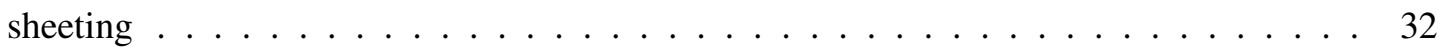

4.4 Temperatures according to Eurocode compared with numerical simulations for Holorib slab ............................ 33 
4.5 Temperatures according to Eurocode compared with numerical simulations for Cofraplus

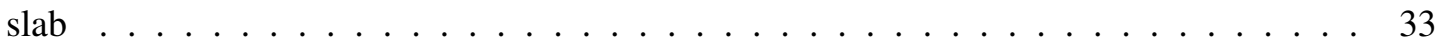

4.6 Rule of Hottel according to [37] . . . . . . . . . . . . . . . . . . . . . . . . 34

4.7 Comparison of temperatures from second Munich test with FEA . . . . . . . . . . . 35

4.8 Derivation of the substitute thermal loading . . . . . . . . . . . . . . . . 37

4.9 Young's modulus of concrete under elevated temperatures (adopted from [26]) . . . . . . 38

4.10 Substitute thermal curvature $\kappa_{\theta, s u b s}$ on full concrete slabs . . . . . . . . . . . . . . 42

4.11 Transverse section through composite slab with schematic isolines for temperature distribution .......................... . . 43

4.12 Determination of location of neutral axis and thermal deformations in transverse direction 44

4.13 System in longitudinal direction . . . . . . . . . . . . . . . . . . . . . 44

4.14 Geometrical notation of steel deckings according to [32] . . . . . . . . . . . . . . . 46

4.15 Substitute thermal curvature in longitudinal direction $\kappa_{\theta, \text { subs }}$ of composite slabs as a function of the effective slab thickness $h_{\text {eff }} \ldots \ldots$. . . . . . . . . . . . . . . . 46

4.16 Substitute thermal curvature in transversal direction $\kappa_{\theta, \text { subs }}$ of composite slabs as a function of the effective slab thickness $h_{\text {eff }} \ldots \ldots$. . . . . . . . . . . . . . . . 47

4.17 System (left) and cross-section (right) of Example 4-1 . . . . . . . . . . . . . . . . . . . 48

4.18 Systems of Example 4-2a (left) and b (right) . . . . . . . . . . . . . . . . . 49

4.19 Stresses in longitudinal direction in central cross-section of Example 4-2a (left) and b

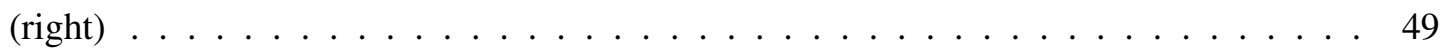

4.20 System (left) and cross-section (right) of Example 4-3: composite beam . . . . . . . . . 50

5.1 Layered element according to Tesar [51] . . . . . . . . . . . . . . . 54

5.2 Stiffness as a function of temperature and strain rate . . . . . . . . . . . . . 54

5.3 Internal forces in a plate under large deformations . . . . . . . . . . . . . . . . 56

5.4 Plan view (left) and cross-section (right) of Example 5-1 . . . . . . . . . . . . . 57

5.5 Influence of the stiffness of the slab on displacement at slab centre . . . . . . . . . 58

5.6 Influence of the stiffness of the slab on membrane forces at centre of slab . . . . . . . 59

5.7 Influence of the stiffness of the slab on bending moments at centre of slab . . . . . . . 59

5.8 Internal forces in restraint beam with non-uniform heating . . . . . . . . . . . . 60

5.9 Crack development on the basis of [55] . . . . . . . . . . . . . . . 61

5.10 Simplified cracking behaviour of a reinforced concrete bar with high (above) and low (below) reinforcement ratio on the basis of [55] . . . . . . . . . . . . . . . 61

5.11 Membrane forces of a single slab panel _. . . . . . . . . . . . . . . . 62

5.12 Reduction of stiffness due to softening of concrete . . . . . . . . . . . . 63

5.13 Tension stiffening on the base of [55] . . . . . . . . . . . . . . . . . . 64

5.14 Effective tension area on the base of [26] . . . . . . . . . . . . . . . 66

5.15 Example 5-2: Options for beam modelling . . . . . . . . . . . . . . . . . 68

5.16 Plastic hinges in steel beams and resulting static system of the edge beams . . . . . . . 69

5.17 Membrane forces of a single slab panel with rigid supports . . . . . . . . . . . . . 70

5.18 Membrane forces of two adjacent slab panels . . . . . . . . . . . . . . . . 71

6.1 Zone method ............................. 74 
6.2 Comparison of damaged zone $a_{z}$ according to DIN EN 1992-1-2, Annex B, Figure B.5 and Equation B.12 [27] . . . . . . . . . . . . . . . . . 75

6.3 Reduced cross-section according to DIN EN 1994-1-2, Annex D [32] and equivalent rectangular cross-section . . . . . . . . . . . . . . . . . 76

6.4 Damaged zone of a Holorib HR51 slab with equivalent cross-section $a_{z, e q}$ and effective

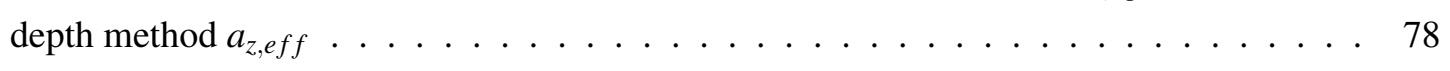

6.5 Damaged zone of effective thickness method . . . . . . . . . . . . 78

6.6 System (left) and cross-section (right) of Example 6-1: load bearing characteristics of

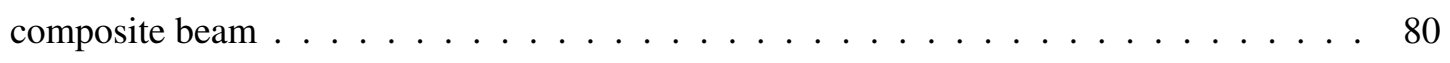

6.7 Load distribution to edge beams according to $[42] \ldots \ldots \ldots \ldots \ldots \ldots$

6.8 Load distribution on longitudinal edge beam at $20^{\circ} \mathrm{C}$ (left) and $540^{\circ} \mathrm{C}$ (right) $\ldots \ldots$. . 82

6.9 Membrane forces with edge beams $540^{\circ} \mathrm{C}$, load transfer to columns . . . . . . . . 82

6.10 Notation of coefficients $b$ and $b_{1}$ for simplified edge beam design according to [42] $\ldots 83$

7.1 Model of first munich test . . . . . . . . . . . . . . . . . 86

7.2 Vertical deformations in $[\mathrm{mm}]$ after 40 minutes, FEA results (above) and test data (below) at Munich test $1 \ldots \ldots \ldots \ldots \ldots \ldots \ldots$

7.3 Principal stresses I in $\left[N / \mathrm{mm}^{2}\right]$ at top surface, Munich test $1 \ldots \ldots \ldots \ldots$

7.4 System (left) and cross-section (right) of edge beam Munich test $1 \ldots \ldots$. . . . . . 89

7.5 Model of second munich test . . . . . . . . . . . . . . . . . 90

7.6 Vertical deformations in $[\mathrm{mm}]$ after 40 minutes, FEA results (above) and test data (below)

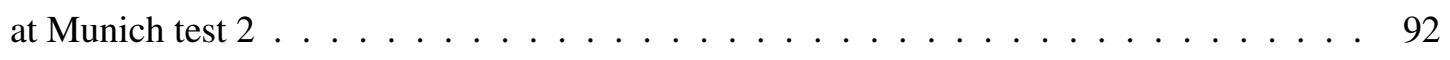

7.7 Principal stresses I in $\left[\mathrm{N} / \mathrm{mm}^{2}\right]$ at top surface, Munich test $2 \ldots \ldots \ldots$

7.8 Model of FRACOF test . . . . . . . . . . . . . . . . . . . . . 93

7.9 Comparison of vertical deformations in $[\mathrm{mm}]$ at $F R A C O F$ test after 105 minutes . . . . 95

7.10 Cross-section for design at slab centre . . . . . . . . . . . 95

8.1 Plan view (above) and cross-section (below) of worked example on the base of [42] . . 97

8.2 Static system and considered points for design . . . . . . . . . . . . . 98

8.3 Cross-section for design in longitudinal direction of steel sheeting . . . . . . . . . 101

8.4 Cross-section for design in transversal direction of steel sheeting . . . . . . . . . . . . 102

8.5 Load distributions on edge beams according to $[42] \ldots \ldots \ldots \ldots$ 


\section{List of Tables}

3.1 Measured concrete material properties in $\left[N / \mathrm{mm}^{2}\right]$ of Munich tests $\ldots \ldots \ldots \ldots$

4.1 View factors for simulations according to the Eurocodes $\ldots \ldots \ldots \ldots$

4.2 View factors for simulation of fire tests . . . . . . . . . . . . . . 35

4.3 Comparison of temperatures after 105 minutes from FRACOF test with FEA . . . . . 36

4.4 Young's moduli of concrete under $20^{\circ} \mathrm{C}$ according to the non-linear stress-strain curves of Eurocode 2 [27] . . . . . . . . . . . . . . . . . . . . . . . . 39

4.5 Reduction factors for the Young's modulus of concrete under elevated temperatures . . . 39

4.6 Substitute thermal loading on full concrete slabs . . . . . . . . . . . . . . . . 41

4.7 Coefficients for the determination of the substitute thermal loading . . . . . . . . . 42

4.8 Substitute thermal loading on composite slabs Holorib HR51 . . . . . . . . . . . . . 45

4.9 Substitute thermal loading on composite slabs Cofraplus $60 \ldots \ldots$. . . . . . . 45

4.10 Coefficients for the determination of the substitute thermal loading in transversal direction 47

4.11 Comparison of analytical method of Section 4.2.2 and FE-method of Section 4.2.3 . . . 48

4.12 Material properties of steel beam in Example $4-3 \ldots \ldots \ldots \ldots$. . . . . . . . 51

4.13 Comparison of results of Example $4-3 \ldots \ldots \ldots \ldots \ldots \ldots$

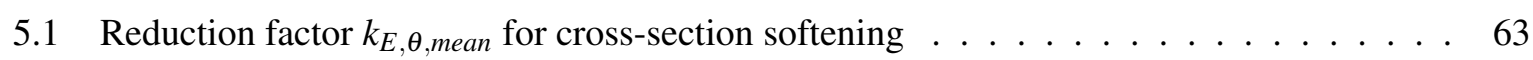

5.2 Comparison of results of Example $5-2 \ldots \ldots \ldots \ldots$. . . . . . . . . . . 69

5.3 Comparison of results of a single slab panel with different support conditions . . . . . 70

5.4 Comparison of results of two adjacent slab panels with different support conditions . . . 72

6.1 Damaged zone $a_{z}$ and minimum distance of the top reinforcement from bottom surface $z_{b} \quad 75$

6.2 Reduction coefficient $k_{c}\left(\theta_{M}\right)$ for concrete at point $\mathrm{M} \ldots \ldots \ldots \ldots$

6.3 Damaged zone in longitudinal $a_{z, \text { eff }}$ and transversal $a_{z, \text { trans }}$ direction of Holorib HR51 and Cofraplus 60 slabs for the effective thickness method . . . . . . . . . . . . . 79

6.4 Comparison of results of Example $6-1 \ldots \ldots \ldots \ldots$

7.1 Temperatures and material properties of the beams of Munich test 1 after 40 minutes . . 87

7.2 Temperatures in slab and substitute thermal load of Munich test 1 after 40 minutes . . . 87

7.3 Temperatures and material properties of the beams of Munich test 2 after 40 minutes . . 91

7.4 Substitute thermal loading on slab of Munich test 2 after 40 minutes . . . . . . . . . 91

7.5 Substitute thermal loading on slab of FRACOF test after 105 minutes . . . . . . . . . 94

8.1 Substitute thermal loading on slab of worked example . . . . . . . . . . . . . 100

8.2 Vertical deformations, internal forces and required top reinforcement, LC1 hot beams . . 100

8.3 Vertical deformations, internal forces and required top reinforcement, LC2 cold beams . 101 


\section{Introduction}

\subsection{Problem specification}

Composite slab systems consist of a grid of primary and secondary steel beams which are connected to thin concrete slabs or composite flooring systems. In this type of construction each material is optimally used according to its best appropriate loading: tensile forces in the steel beams and compression in the concrete. This enables bridging of long spans without distracting columns by having very small construction depths. In case of fire, however, the steel beams rapidly lose their strength if they are not protected against the heat. For that reason, in classical fire protection design every steel beam has to be covered by gypsum boards, sprayed concrete, intumescent coatings or other costly measures that prevent them from immoderate heating. It is not always necessary to protect every steel beam in order to prevent collapse. At ambient temperature a slab mainly transfers load by bending. If unprotected secondary beams within a slab field lose strength during a fire, large deformations occur which activate membrane forces in the slab. The load can then be transferred by an alternative path and the unprotected beams are no longer necessary. In the literature this kind of load transfer mechanism usually is called tensile membrane action. At the centre of a slab panel tensile membrane forces occur and around the perimeter compressive forces as shown in Figure 1.1.

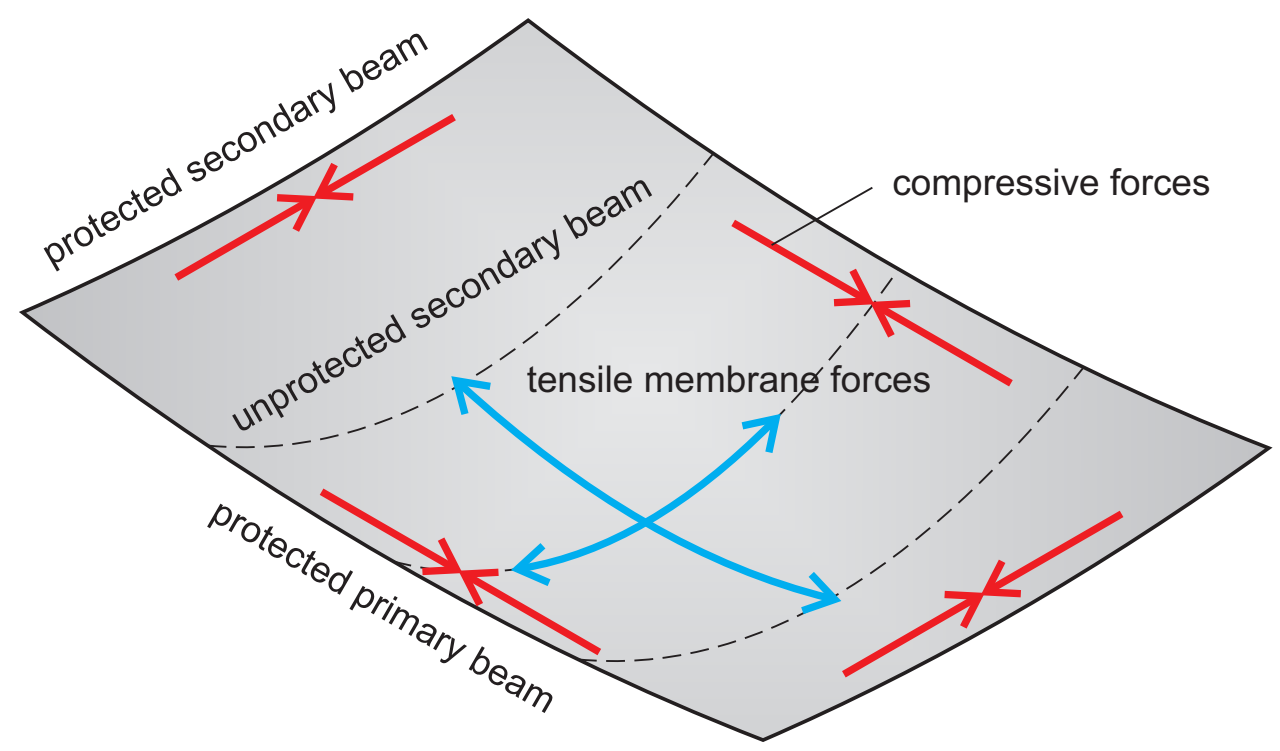

Fig. 1.1: Tensile membrane action of a single slab panel

The horizontal forces are in equilibrium therefore no horizontal restraint of the slab panel is necessary. Only the vertical forces have to be transferred into the columns. For that reason the edge beams have to be protected. The application of membrane action in design procedures of slab systems for the case of fire has huge economic potential since a large amount of the expensive fire protection measures can be avoided. 


\subsection{Objective and layout of the thesis}

Several research projects and academic research works have been conducted to investigate membrane action, however, none of the available approaches can satisfy all of the requirements for use in practice. All fire tests, that were performed in these projects, could show that membrane action enhances the load bearing capacity of composite beam slab systems in case of fire considerably. In some of the tests, however, integrity failure occurred in terms of a gaping crack nearby an intermediate beam between two slab panels. Approaches are available to avoid this kind of failure but they need to be improved. With the method presented in this work, a required reinforcement amount can be calculated to solve this problem.

Different methods have been proposed so far that use membrane action for design. Advanced calculation models using general finite element programs such as Abaqus and Ansys are the most exact and unlimited options, but these are also complicated, fault-prone and time-consuming. The two main reasons for these problems are: firstly two separate simulations (thermal and mechanical) have to be performed, and secondly, complex non-linear material laws have to be included. Specialised software such as Safir, Vulcan or SlabFem faces some of these problems by implementing the material laws as a general preset, however, two separate simulations still have to be performed and the non-linearities still induce enormous computing times. Significantly, the common design procedures for reinforced concrete under a combination of internal bending moments, shear and normal forces cannot be used with non-linear finite element analyses, and so the design process for a new structure is very time-consuming.

Simple calculation models can be applied faster and more efficiently, particularly if these are presented as design tables such as those contained in design methods from the $S C I$ and the FRACOF project, or are implemented in specialised software such as TSLAB. The available models include assumptions which clearly have to be revised, they neglect some important aspects and are very limited in their application spectrum. Currently no really satisfactory approach is available to determine the maximum allowable vertical deformation of a slab, although in all current simple calculation models this is vital in order to predict the load-bearing capacity. The influence of the edge beams on the force distribution is not taken into account in any of these methods, furthermore, the interaction with adjacent slab panels is neglected. While this may be considered as an implicitly conservative assumption, it can lead to the formation of large cracks, causing failure of integrity and eventually of structural resistance.

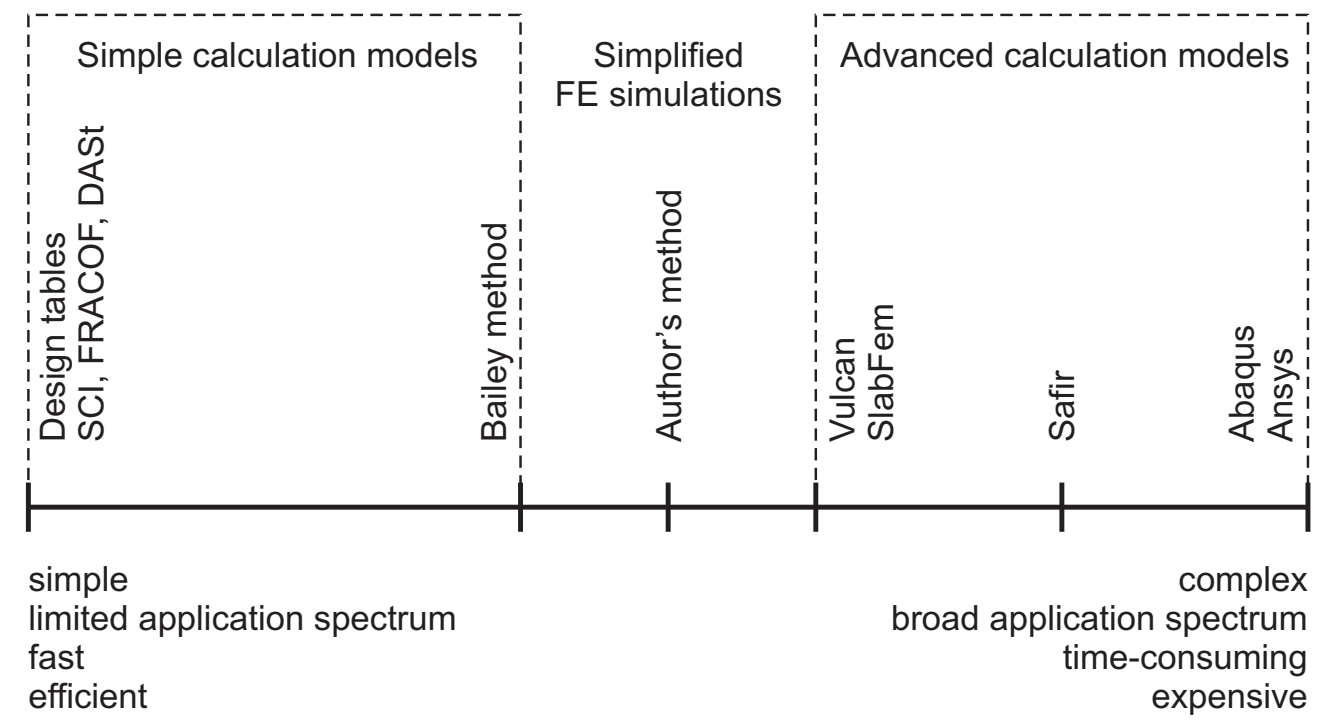

Fig. 1.2: Positioning of the research results 
Presently no procedure is available that satisfies the requirements of a safe and efficient design. Simple calculation models are too limited in their application spectrum and neglect important aspects and advanced calculation models are too complicated, time-consuming and therefore expensive in application. The objective of this thesis is to close this gap. The positioning of the work in the research field of membrane action is visualised in Figure 1.2.

This thesis presents a new design procedure with following specifications:

- The assumptions in the method shall follow the Eurocodes where possible in order to ensure that the method conforms to national regulations in every country that has adopted the Eurocodes.

- No thermal analysis is necessary by using the given tables and approximation functions. The thermal expansion is taken into account by a substitute thermal loading.

- No material non-linear calculation is necessary. The material non-linearities are substituted by reduced stiffness.

- A geometrically non-linear calculation is required in order to activate membrane forces.

- The internal forces can be calculated with ordinary finite element programs.

- The amount of reinforcement is designed in the same way as at ambient temperature with internal forces and cross-section design procedures.

- No limitations are given in terms of geometry and material that are used in common constructions.

- Only the heating phase can be considered, no cooling phase.

In Chapter 2 of this thesis the research projects, that were conducted on the topic of membrane action so far, are summarised and the currently available design methods are discussed. In Chapter 3 experimental investigations are described. The data of these tests are used later on in this work. Chapter 4 deals with the heat transfer into the considered structure and the effects of the heating. It will be explained how the effects of the heating can be replaced by a substitute thermal loading. In Chapter 5 modelling recommendations are given and it is described how material non-linearities can be avoided. Chapter 6 provides procedures which can be used for designing the structure with the results of the simulations that are performed with the method of the previous chapters. In Chapter 7 the proposed method is validated on the fire tests of Chapter 3. Chapter 8 contains a worked example that will illustrate the application of the proposed method. In Chapter 9 the thesis is summarised and recommendations for further research work are given. 


\section{State of research}

\subsection{Research projects}

The first findings on membrane action were made coincidentally. In 1990 a fire broke out at a construction site of an office building in London. The steel structure and composite slabs were already erected but no fire protection had yet been installed. The fire burned for four hours and temperatures reached more than $1000^{\circ} \mathrm{C}$ but the building did not collapse. Only large deformations could be observed. A detailed description of the observations is given in [44].

After this incident a series of research projects were launched including the Cardington-project. Inside an abandoned zeppelin hangar in Cardington (GB) an eight-storey composite building was erected under the lead of the British Building Research Establishment (BRE). Between 1995 and 2003 seven fire tests were performed inside the building. In [44] a summary of the first six tests performed between 1995 to 1996 can be found. A more detailed description is given in the report of the Cardington-project [11]. Test seven was conducted in 2003 and is documented in [12]. Structural collapse never occurred although in most of the tests all of the steel beams were left unprotected. In this project it could be identified for the first time that a considerable increase in load bearing capacity is caused by membrane action. The results of the project finally led to a design guide [44] published by the British Steel Construction Institute (SCI). The design tables in this guide are mainly based on the work of Colin Bailey who worked out a simplified model to predict the load bearing capacity of the slabs. In the literature this model is called Bailey-BRE method or simply Bailey method. The method is discussed in Section 2.3.1.

A further research project that is worth to mention was called Fire Resistance Assessment of Partially Protected Composite Floors (FRACOF). The main objective of the project was to distribute the knowledge of membrane action outside of Great Britain in Europe. One full scale fire test was performed within this project in 2008. A single slab panel was exposed to a fire whose temperature-time curve followed the standard fire curve according to ISO 834. After 105 minutes a large crack occurred at the slab centre and the test was stopped shortly after. The test confirmed that composite slab systems do not collapse even if some of the secondary beams are unprotected but the tested slab failed in terms of integrity. The test will be described in more detail in Section 3.2 since the results are used later on in this thesis to validate the approaches. A design guide was published [48] and the design procedure was implemented in a design software. The engineering background with test details can be found in [49]. In this project no significant new investigations were made. The content of the design guide largely corresponds with the guide of the $S C I$ [44]. No suggestions were made to avoid the integrity failure but the impact of the project was considerable, especially in France and Switzerland. A Swiss design book in print refers to the design tables of the FRACOF design guide [48].

In 2011 the federal research project Nutzung der Membranwirkung von Verbundträger-Decken-Systemen im Brandfall (Utilisation of membrane action for the design of composite beam-slab-systems in fire) was completed by the Technische Universität München in cooperation with the Leibniz Universität 
Hannover. The main objective of this project was to enable the use of membrane action in Germany. In addition, some details were investigated including the behaviour of protected intermediate beams between two slab panels. As part of the project, two large scale fire tests were conducted in Munich while numerical investigations were performed in Hannover. The author of this thesis did the research on behalf of the TU Munich. The tests are described in detail in Section 3.1. Design tables were calculated in the project and construction details were suggested. The design tables base on parameter studies made by using the software Vulcan which is explained in Section 2.2.2. New tables were necessary due to the fact that no satisfactory available design method could be found. The report of the research project was published by the German steel construction association (DASt) [42]. One of the fire tests brought up an entirely new issue that had not been considered in this field of research so far. A large crack occurred nearby the intermediate beam only 19 minutes after the test started (see Fig. 2.1). The entire reinforcement in the crack ruptured. The slab did not collapse but lost its integrity. A pragmatic solution was suggested to face this problem which is discussed in Section 2.3.

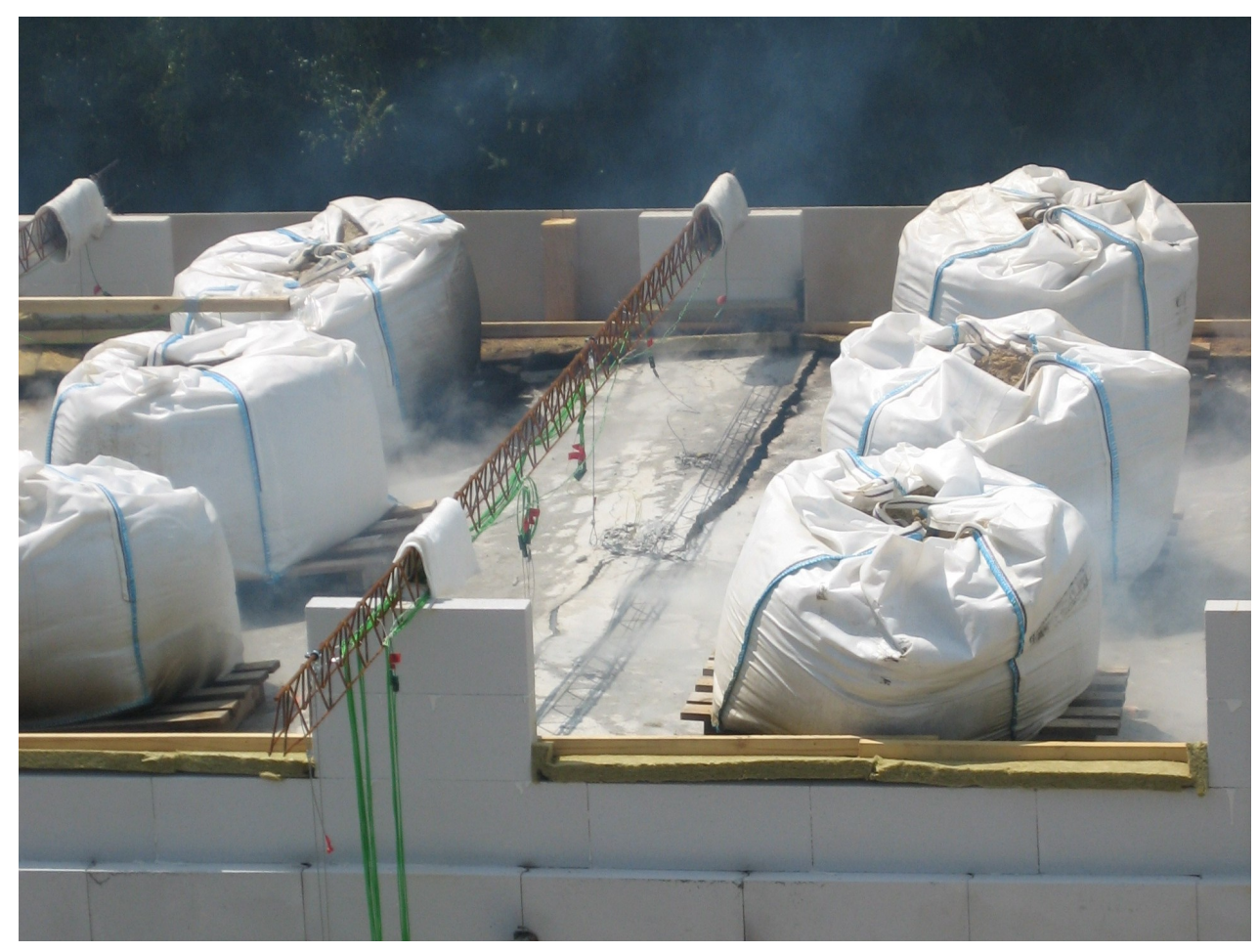

Fig. 2.1: Gaping crack nearby the intermediate beam in first Munich test

Further large scale fire tests on membrane action were performed for example 2008 in Mokrsko (CZ) [54], 2010 in Belfast (GB) [52] and 2011 in Veselí (CZ) [53]. Although the test in Veselí was not part of a project dealing with membrane action, it nevertheless showed some interesting results in that respect. To investigate the robustness of joints of composite beams in fire conditions, a test on a four bay composite slab with unprotected steel beams was conducted. Nearby one intermediate beam a huge gaping crack occurred in a similar location and crack pattern as in the first test in Munich. The structure did not collapse but again a slab failed in terms of integrity. Small scale tests were performed for instance by Colin Bailey [9], Samantha Foster [35] and Anthony Abu [1]. 


\subsection{Advanced calculation models}

Since the processing power of personal computers has risen exponentially over the last decades, complex and computationally intensive numerical simulations have become standard in all fields of structural engineering. Material properties for steel and reinforced concrete are particularly well investigated. A lot of research works has also been carried out to investigate how elevated temperature material behaviour can be implemented in numerical simulations. With the adoption of the Eurocodes [24], [25], [27], [28], [29], [30], [32], [33] for the first time regulations are available that allow to use advanced calculation models including numerical simulations for the design of structures in case of fire.

\subsubsection{General numerical simulations}

In principle, a simulation of a structure for elevated temperatures is split into three parts: fire simulation, thermal analysis and mechanical analysis. In the fire simulation the combustion of the flammable material inside the building is simulated to predict the temperature time curve of the gas surrounding the structure. Fire scenarios are usually simulated with so-called zone models or computational fluid dynamics (CFD), not with finite elements (FE) like the other two parts. This part of the simulation is an entirely separate field of research and is currently not fully developed to be used for practical application. Therefore the internationally agreed ISO 834 standard fire curve is mostly used for the gas temperature in simulations of structures in fire. This fire curve is usually called ISO fire. Detailed information on this topic can be found for example in the book of Ulrich Schneider [46].

The thermal analysis simulates the heat transfer from the surrounding hot gas into the structure and the distribution inside the structure. The heat transfer into the structure is carried out by convection and radiation. The convection mainly depends on the fire scenario. The intensity is driven by gas movements at the surface of the structure. The higher the gas movements are inside a fire compartment the greater is the convective part of the heat transfer. The radiative part depends on the colour of the surface. Absolutely black surfaces absorb radiation perfectly and absolutely white surfaces perfectly reflect it. Inside the structure the heat is transferred by conduction. How much heat is transferred or absorbed depends on the thermal conductivity, the mass and the specific heat of the material. More details on heat transfer can be found in [45]. A summary that is more specific for structural engineering is given in [46]. The specifications of the Eurocodes and the assumptions used in this thesis are described in Section 4.1.

The mechanical analysis simulates the response of the structure on the heating combined with mechanical loads such as dead and live loads. The response appears in terms of deformations, stresses and strains. In this part of the simulation the reduced stiffness and strength of the material has to be considered as well as the thermal expansion. All material non-linearities have to be combined with geometrical non-linearities in order to take into account the large deformations and membrane forces. The Eurocodes contain specifications for material properties of steel and concrete under high temperatures. Stress-strain curves are given for different temperature levels for both materials. The curves for steel can be relatively easily implemented in finite element models. Steel shows a similar behaviour under tension and compression. It behaves ideally elastic before it starts to yield. Yielding follows simple plasticity rules under uni and multiaxial loading. The best known plasticity rule of steel is the von Mises theory. The finite element software only has to interpolate between the curves of the input temperature levels. The mechanical analysis is disproportionately more difficult for concrete. The behaviour under compressive forces is completely different to under tension. For the compressive part stress-strain curves 
are given in the Eurocodes [27], [32]. For the tensile part DIN EN 1992-1-2, 3.2.2.2 [27] only says: "The tensile strength of concrete should normally be ignored (conservative)." This would mean that in numerical simulations the tensile stiffness of the concrete elements needs to be zero which may lead to numerical instability. For the simulation of membrane action it is not conservative to neglect the tensile strength and stiffness of concrete. Lower stiffness leads to higher load bearing capacities as explained in Section 5.2.2. A big influence on the tensile stiffness is the reinforcement that acts compositely with the cracked concrete under tension. The concrete does not crack completely but still provides stiffness between the cracks. This behaviour is generally called tension stiffening. Approaches are available which implement tension stiffening in non-linear numerical simulations [51]. Usually, the descending branch of the tensile part of the stress-strain curve is modified but the required parameters are only available for ambient temperature and not for elevated temperatures. A further problem is the multiaxial behaviour of concrete in plasticity. Concrete shows no linear elastic behaviour like steel from the very beginning of loading. It also shows no real plasticity but brittle behaviour when the material strength is reached. The behaviour in the considered direction of loading is dependent on the loading in perpendicular directions. For example, the compressive strength is much higher if compressive stresses act perpendicular to it than if tensile stresses would do. A material model that is often used in numerical simulations is the Mohr-Coulomb failure criterion as shown in Figure 2.2. Stress combinations that are located inside of the shown body can be borne by concrete. The surface of the body forms the limit to cracking and crushing of the concrete. This material model was developed for ambient temperature and has not been proven for elevated temperatures.

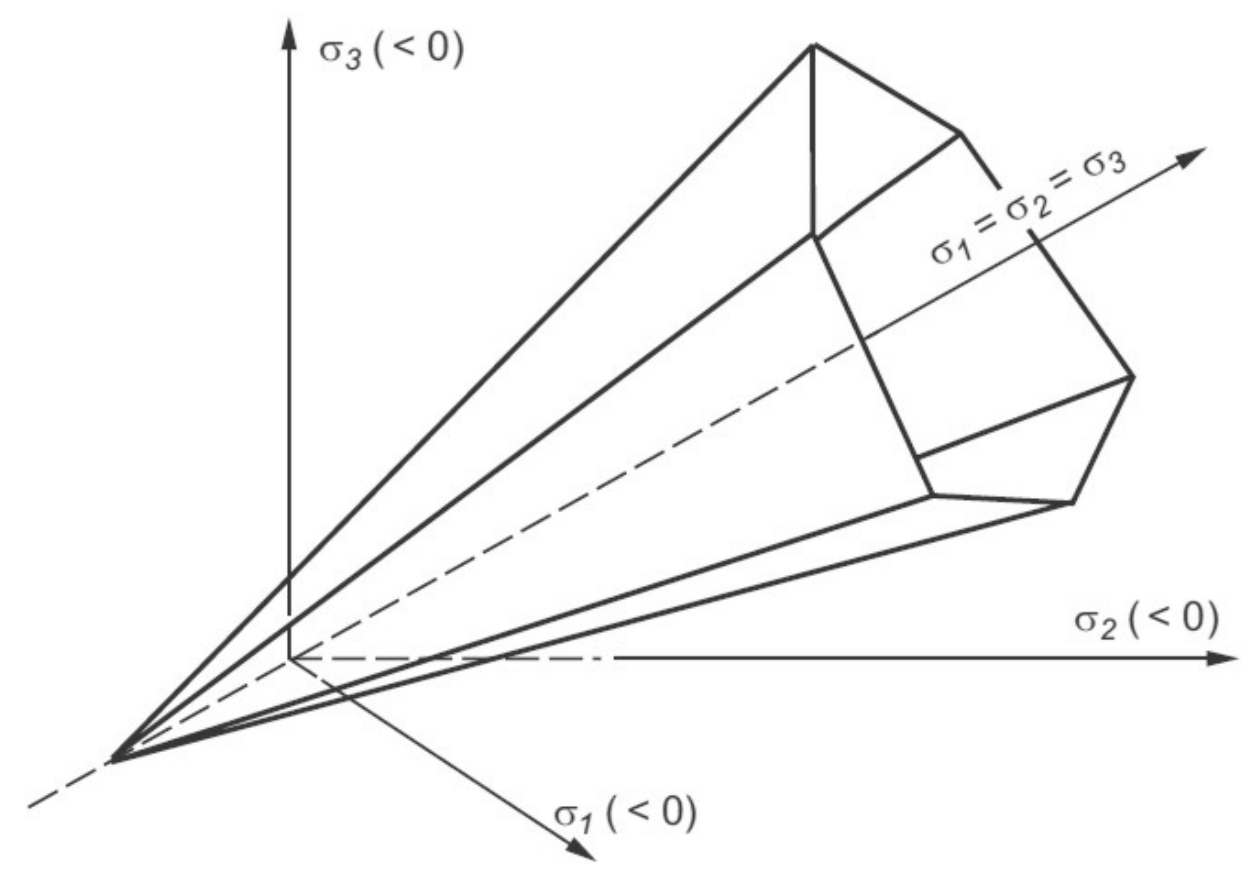

Fig. 2.2: Mohr-Coulomb failure criterion [55]

The cracking of concrete is a challenge to be realistically taken into account in non-linear numerical simulations. In reality, a crack appears as soon as the tensile strength of the concrete is reached. The stiffness of the structure reduces at the location of the crack. If discrete cracks were to be modelled in numerical simulations, it would mean that the finite elements need to be split at the location of the crack. Since it is not known before the simulation where the cracks will occur, the calculation routine of the 
finite element software has to be able to automatically split elements. Such discrete crack models are often called XFEM and are currently in development [47]. They are not yet implemented even in the high-end finite element software packages like Abaqus and Ansys. So-called smeared crack models are therefore most commonly used at the moment. In these models, only the stiffness of the finite element is reduced as soon as the tensile strength is reached. A detailed description of these models is given in [51].

Material non-linear numerical simulations are often not very useful for designing new structures. Every cross-section, material strength, amount of reinforcement etc. has to be defined before the simulation. The result of the simulation then is just convergence if every part of the input structure was defined sufficiently strong or divergence if not. Obtaining an adequate structure is a time consuming process that is not economically viable. For ambient temperature, the usual procedure to design a structure is much more efficient: pre-choosing the cross-sections, determining the internal forces with linear calculations and designing every cross-section for these forces. A further general problem is the complex load bearing behaviour of a reinforced concrete cross-section under combined loading with bending moments, normal and shear forces. For this behaviour cross-section design procedures have been developed which are standard in every design code. This behaviour is very difficult to include in non-linear finite element simulations, particularly the interaction with shear forces.

The descriptions above show that the requirements of the finite element software and of the ability of its user are rather high. The input of the numerical model is complicated and fault-prone. The highly non-linear temperature dependent material laws have to be combined with geometrical non-linearities, resulting in huge computing times and convergence problems. Even with sophisticated processors and relatively small models, computing times easily reach several days. Frequently after a couple of days of computing the solver reports numerical instability. It might therefore take weeks or even months to find and solve a problem and get one single result. The most common software packages in this highend area are Abaqus and Ansys. Both are general finite element programs that are used for research. Commercial applications are mainly in the field of mechanical engineering. The pre and post processors are therefore adjusted to their requirements. The programs only output stresses, strains and deformations. Inputs and outputs that are common in structural engineering such as line loads, different load cases, bending moments and supporting forces are not requested by mechanical engineers and therefore not implemented in the software. Since all the design procedures in structural engineering, particularly for reinforced concrete structures, are based on internal forces not on stresses, the user has to integrate the stresses manually in order to get internal forces and to be able to use the design procedures.

\subsubsection{Specialised software}

To face at least some of the problems mentioned above, specialised software has been developed to simulate composite slabs under elevated temperatures. The user shall not need to think about material laws but only has to input the geometry of the structure. Everything else is preset in the software. The most highly developed specialised software is called Vulcan ([40], [41]) which was developed at the University of Sheffield and is continuously enhanced. It is primarily dedicated to model membrane action of composite slabs but can also be used for general simulations of steel and composite structures in fire. Presently, Vulcan only includes the mechanical analysis. The thermal analysis has to be performed with other programs. The program generally includes the temperature dependent material properties of the Eurocodes. Steel beams are taken into account with beam elements that are eccentrically connected to 
the concrete slab. The slab is modelled with layered shell elements. Each layer can be given a certain temperature to model the temperature distribution inside the slab. Single layers can also be used as reinforcement. A smeared crack model is used for the cracking behaviour. Multiaxial behaviour is used with a model according to Kupfer. A description of this model and references can be found for example in [40] and [51]. Some simplifications are made in Vulcan. The behaviour of concrete before cracking or crushing is assumed to be linear elastic. After crushing the stiffness is assumed to be zero. It cannot be found in the literature whether tension stiffening is included. Shear forces are not taken into account. These simplifications were probably made to avoid numerical instabilities. Structures in fire can be modelled very easily with Vulcan and reasonable results are obtained, however, it does not solve the general problems of material non-linear simulations. It also requires long computing times, all the material strengths and reinforcement amounts have to be set before the calculation and the cross-sections cannot be designed with internal forces and a separate thermal analysis has to be done.

A recently developed specialised program is called SlabFem. It was written by Can Tesar in the course of his dissertation [51]. Many of his approaches are similar to Vulcan. The slab is modelled with layered shell elements and the steel beams as eccentrically connected beam elements. Most improvements were made in the element formulations and solver strategies but also more obvious details were improved. It includes the whole non-linear stress strain curves of concrete from the Eurocodes. Also the thermal analysis can be done with SlabFem, however, it cannot be found in the literature if the software has been commercialised. It is also not known if it includes a useful pre and post processor. The computing times have not been tested by the author but they might be long since the non-linear material laws are included. Shear forces are also not taken into account.

Another software in this field worth mentioning is called Safir [36]. It was developed at the University of Liege for the general modelling of steel, concrete and composite structures in case of fire and can be used for the simulation of membrane action. With some differences in details, it shows the same advantages and disadvantages as Vulcan and SlabFem.

It can be summarised that specialised software simplifies the advanced calculation models considerably but does not solve the problem of very long computing times. The design of a new structure is also very time-consuming with these programs. They are therefore mainly used for research so far. Although they can be used much more efficiently than general programs, the suitability for commercial application is limited. The savings that are achieved by leaving some of the secondary beams unprotected might be completely dissipated in the design process.

\subsection{Simple calculation models}

In order to avoid the problems of numerical simulations as described above and to enable a very simple application of membrane action, several simple calculation models have been suggested since the Cardington project. Two of them are focused in more detail in this section. Further approaches were made by Neil Cameron [15], [16], [17] and Anthony Abu [1] who tried to apply analytical approaches with energy methods such as the Rayleigh-Ritz model. Internal forces of slabs can be determined with these models but they are limited to the assumed boundary conditions. Cameron only considered horizontally restrained and rotationally free boundary edges and Abu both horizontally and rotationally free edges. The solution of these approaches does not lead to simple equations but has to be done by numerical integration. Since complex numerical solving procedures need to be performed in the methods of Abu 
and Cameron, results can be achieved more easily with finite element simulations.

Ian Burgess and Anthony $\mathrm{Abu}$ [2] proposed an approach that takes into account the load bearing capacity of the edge beams. They assume that a slab panel can be folded along yield lines with different folding mechanisms. The mechanism with the lowest load bearing capacity is decisive. This approach is suitable to check the load bearing capacity of the edge beams. This check is disregarded by most available design methods but it is crucial. If the edge beams are not designed sufficiently strong, they will fail before membrane action can be built up. A very simple approach for designing the edge beams is provided in Section 6.2.

\subsubsection{Bailey method}

The best-known and most commonly used model for designing composite slabs in case of fire is the method of Colin Bailey [4], [5], [6], [7], [8]. The Bailey method is based on the yield line theory which has been developed for slabs under ambient temperature and small displacements. The yield line theory assumes four rigid plates that are pin-jointed at their edges. The load bearing capacity in the classical theory is reached as soon as plastic bending hinges form along the yield lines. In contrast to this theory, Bailey assumes a failure mode with a crack in the centre of the slab along the short span as shown in Figure 2.3. He explains this assumption by referring to his observations in fire tests. The assumed forces along the yield lines and the crack are shown in Figure 2.3 on the right hand side. The force along the crack is calculated with the yield strength of the reinforcement.
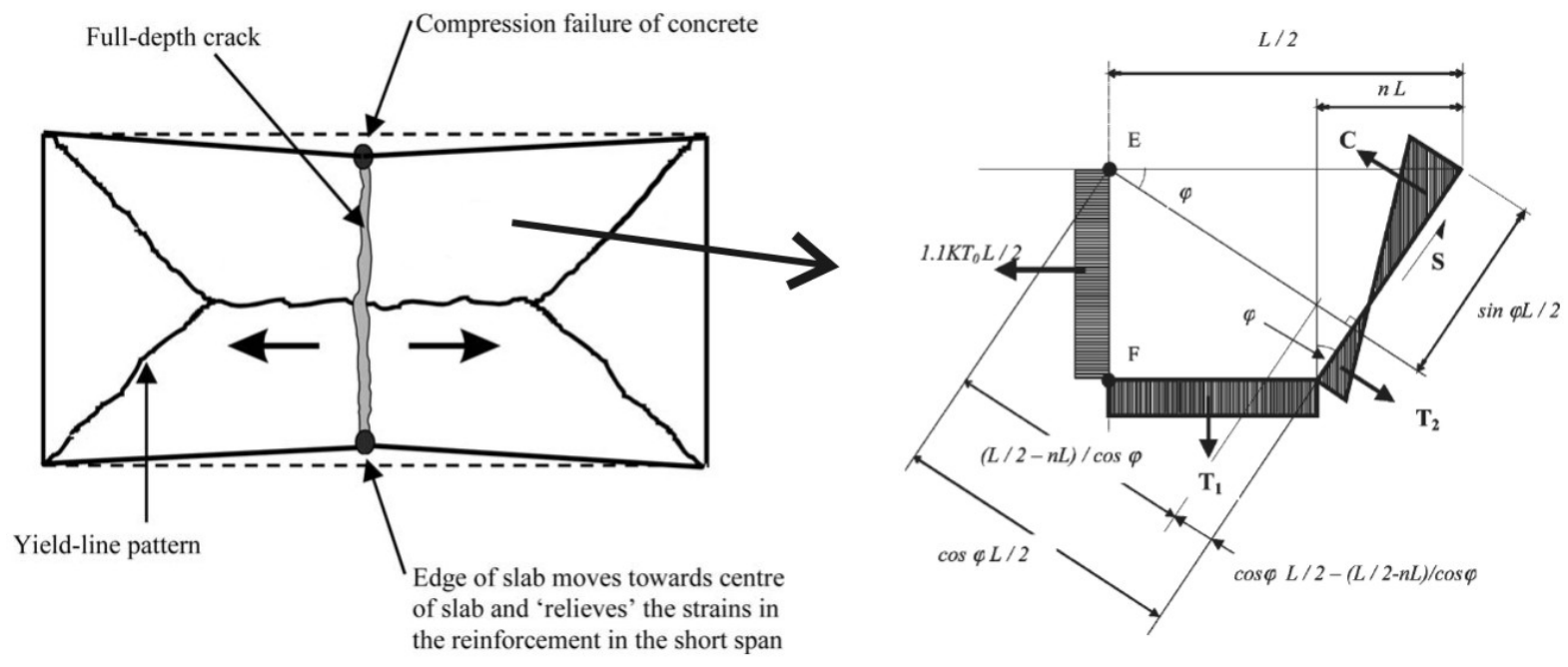

Fig. 2.3: Failure mode (left) and forces along yield lines (right) according to [5]

The load bearing capacity is calculated by multiplying these forces with a given vertical displacement $w$. The maximum allowable displacement must be determined separately by other assumptions. Bailey proposes the following equation:

$$
w=\frac{\alpha_{c} \cdot \Delta T \cdot l^{2}}{\psi \cdot 8 \cdot h}+L \sqrt{\frac{3}{8} \cdot \frac{0.5 f_{y}}{E_{s}}}
$$

where:

$\alpha_{c} \quad$ is the thermal expansion coefficient of concrete; 


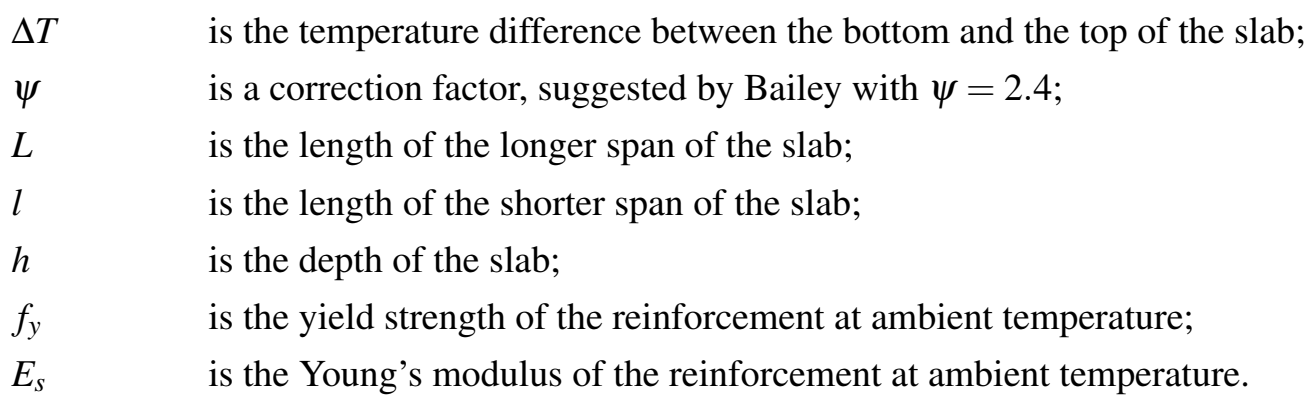

The first part of Equation (2.1) is the vertical displacement of a simply supported beam with a length of the shorter span of the slab and which is bowing due to a thermal curvature. It includes a correction factor $\psi$. The second part is the vertical displacement of a rope with a length of the longer span of the slab with a mechanical strain of $50 \%$ of the yield strain of the reinforcement. From the mechanical point of view, these two parts do not fit together. Two displacements of two different mechanical systems are combined. Equation (2.1) was calibrated on a small number of tests with the correction factor and the assumption of $50 \%$ of the yield strain. This problem is mentioned in the literature but few alternative approaches have been published so far. One alternative suggestion is given by Neil Cameron [16]:

$$
w=\frac{l}{\pi} \sqrt{4\left(\varepsilon_{u}+\alpha_{c} \Delta T\right)}
$$

He slightly modified Bailey's approach. Only one beam with the length of the shorter span of the slab $l$ is considered. The shape of the deformation is not parabolic like in Bailey's approach but sinusoidal. The thermal gradient has no correction factor and the mechanical strain is assumed with the ultimate strain of the reinforcement $\varepsilon_{u}$. Cameron's approach has not been validated by tests. The author doubts that it is generally possible to find a simple equation to determine the maximum vertical displacement. It is probably not sufficient to describe the complex behaviour of a two span slab by only considering a simple beam. A variety of other influencing factors have to be taken into account, for instance geometry of the slab panel, horizontal and rotational restraint of the edges, deformation of the edge beams, orthotropy of the composite slab, tension stiffening etc. This complexity of the system is probably the reason why no better approach has been found so far. Due to the lack of alternatives the approach of Bailey is used in all design guides that are based on his model. For example, his method is included in the SCI guide in Great Britain [44], the FRACOF design guide in France [48] and also in guides in Switzerland and New Zealand.

The Bailey method neglects important aspects for a comprehensive design. The supports around the edges are considered ideally rigid and are not restrained horizontally and rotationally. This means that the influence of the steel beams at the edges is not taken into account. The deformation of these beams, the thermal elongation and the interaction with adjacent slab panels have an effect on the distribution of the forces in the slab as discussed in detail in Section 5.3. As seen at the first test in Munich and the test in Veselí, gaping cracks at intermediate beams can occur in cases where the interaction with adjacent slab panels is neglected. It is important to consider the deformation of the edge beams since forces concentrate at the columns at the corners when the beams deflect. The thermal elongation has to be taken into account since it is restrained by the concrete and therefore causes additional tensile forces in the slab. In addition, it is not obvious where the largest stresses in the slab occur. Bailey's assumption, that the largest stresses are located along the yield lines and inside a crack at the centre of the slab, is only valid for unrestrained slab panels with rigid supports. 


\subsubsection{DASt design tables and construction details}

Due to the uncertainties of the available methods, new design tables and construction details were worked out in the the German DASt research project [42]. The tables are based on a parameter study with Vulcan in which the edge beams were included. To avoid the gaping cracks at intermediate beams, it was suggested to lay in a crack control reinforcement. According to the study, the crack could become that large since not enough reinforcement was available. As soon as the tensile strength of the concrete was reached in the test, the crack occurred and all the forces were transferred to the reinforcement. The reinforcement was too weak to bear these forces and to distribute the cracks. Strains were concentrated in a very small length along the reinforcement and it ruptured. The crack was then able to open without any restraint. In general, if more reinforcement is available than required for the tensile strength of the concrete, further cracks can be induced, the strains do not concentrate in the first crack and thus, much larger deformations are possible without damage.

In the project it could not be found a way to determine the internal forces in the slab. These forces are necessary in order to calculate the required reinforcement amount and the resulting crack widths. The study suggests therefore to determine a minimum reinforcement for restraint with a maximum calculated crack width of $w_{k}=0.2 \mathrm{~mm}$ according to DIN EN 1992-1-1, 7.3 [26]. With the simplification that pure tension is occurring, a minimum reinforcement area can be calculated as follows:

$$
A_{s, \min }=\frac{f_{c t m} \cdot h_{c, e f f} \cdot b}{\sigma_{s}}
$$

Where:
$f_{c t m} \quad$ is the tensile strength of the concrete;
$h_{c, e f f} \quad$ is the height of the effective tension area, should be assumed at least with half of the slab depth;
$b \quad$ is the breadth of the effective tension area;
$\sigma_{s} \quad$ is the maximum allowable stress in the reinforcement according to DIN EN 1992-1-1, Table 7.2N.

Calculating the reinforcement amount with Equation (2.3) ensures that all forces can be accommodated when the tensile strength of the concrete is reached. The limitation to a bearable stress lower than the yield stress is used to keep the strains and the crack widths small. This reinforcement shall prevent large cracks and increase the rotational capacity of the concrete cross-section above the intermediate beams by distributing the cracks. The combination of forces caused by mechanical loads and restraint, however, cannot be taken into account with this approach and the design tables of the project are limited. Only slab dimensions between $5.0 \times 7.5 \mathrm{~m}^{2}$ and $7.5 \times 10.0 \mathrm{~m}^{2}$ are covered. Two load cases are considered, one with a small live load of $2.0 \mathrm{kN} / \mathrm{m}^{2}$ and one with a high load of $5.0 \mathrm{kN} / \mathrm{m}^{2}$. And like all available simplified methods, it is limited to rectangular slab panels.

It can be summarised that simple calculation models are more efficient in application than advanced calculation methods. The slabs can be designed with simple formulas or design tables and no complex non-linear finite element analysis has to be performed. Models currently available are, however, limited in their application spectrum and include assumptions that can lead to unsafe structures. A new method is proposed in this thesis that offers a broader application spectrum and takes into account the important aspects that are neglected by the available simple models. 


\section{Experiments}

The behaviour of structures can be investigated very cost-efficiently with finite element analyses. The model can be changed easily and a lot of parameters can be considered. Experiments, however, have remained indispensable. These are necessary, on the one hand, to investigate material properties which are required for the simulations and, on the other hand, to calibrate numerical models and validate the simulations. The complex behaviour of reinforced concrete in case of fire needs to be further investigated with experiments in particular.

The data of three large scale fire tests are used in this work. Two of them were performed in Munich by the author, one with a full concrete slab and the other with a composite slab with re-entrant trough profile steel sheeting. In order to be able to investigate composite slabs with open trough profile steel sheeting, the data of the test within the FRACOF project are also used in this work.

\subsection{Munich fire tests}

Two full scale fire tests on membrane action were performed within the DASt research project. The main objective was generating data which can be used to calibrate numerical models and design methods. One focus of the experiments was the behaviour of intermediate beams between two slab panels which was not well-investigated so far. The tests were conducted at the fire testing laboratory of the Technische Universität München in Dachau. The first test was performed on 7th July and the second test on 3rd September 2010. Detailed descriptions and test results can be found in the report of the project [42]. The details of interest for this work are summarised.

\subsubsection{Test arrangement}

A typical application of membrane action is in office buildings and similar multi storey structures. The test arrangement was chosen to represent slabs in such buildings. The specimens both consisted of two slab panels with overall dimensions of $5.0 \mathrm{~m}$ by $12.5 \mathrm{~m}$ and a total depth of $12 \mathrm{~cm}$ as shown in Figure 3.1. These were supported by hot rolled I-beams and six columns at the corners of the panels. The edge beams and the intermediate beam were protected by intumescent coating. The secondary beams inside each panel were left unprotected. The columns were not part of the investigation and consisted of reinforced concrete. The aspect ratios of the panels were chosen differently. The reason was to investigate the influence of the aspect ratio on membrane action and to represent a typical arrangement in an office building with a larger panel in the regular grid and a smaller panel at the ends.

Two tests with slightly different arrangements were performed. Only the orientation of the secondary beams, the flooring system and the intumescent coating system were varied. The first specimen was built with a $5 \mathrm{~cm}$ thick lattice girder precast slab with in-situ concrete topping, and the second one with a composite slab type Holorib HR51 as shown in Figure 3.2. The cross-sections and reinforcement amount were designed for ambient temperature according to DIN EN 1994-1-1 [31]. The mechanical 
loading was chosen for a typical office building with an additional dead load of $1.5 \mathrm{kN} / \mathrm{m}^{2}$ and a live load category B1 of $2.0 \mathrm{kN} / \mathrm{m}^{2}$ according to DIN EN 1991-1-1 [23]. The load ratios were chosen very close to $100 \%$ to prevent the structure from resisting the fire due to oversizing.
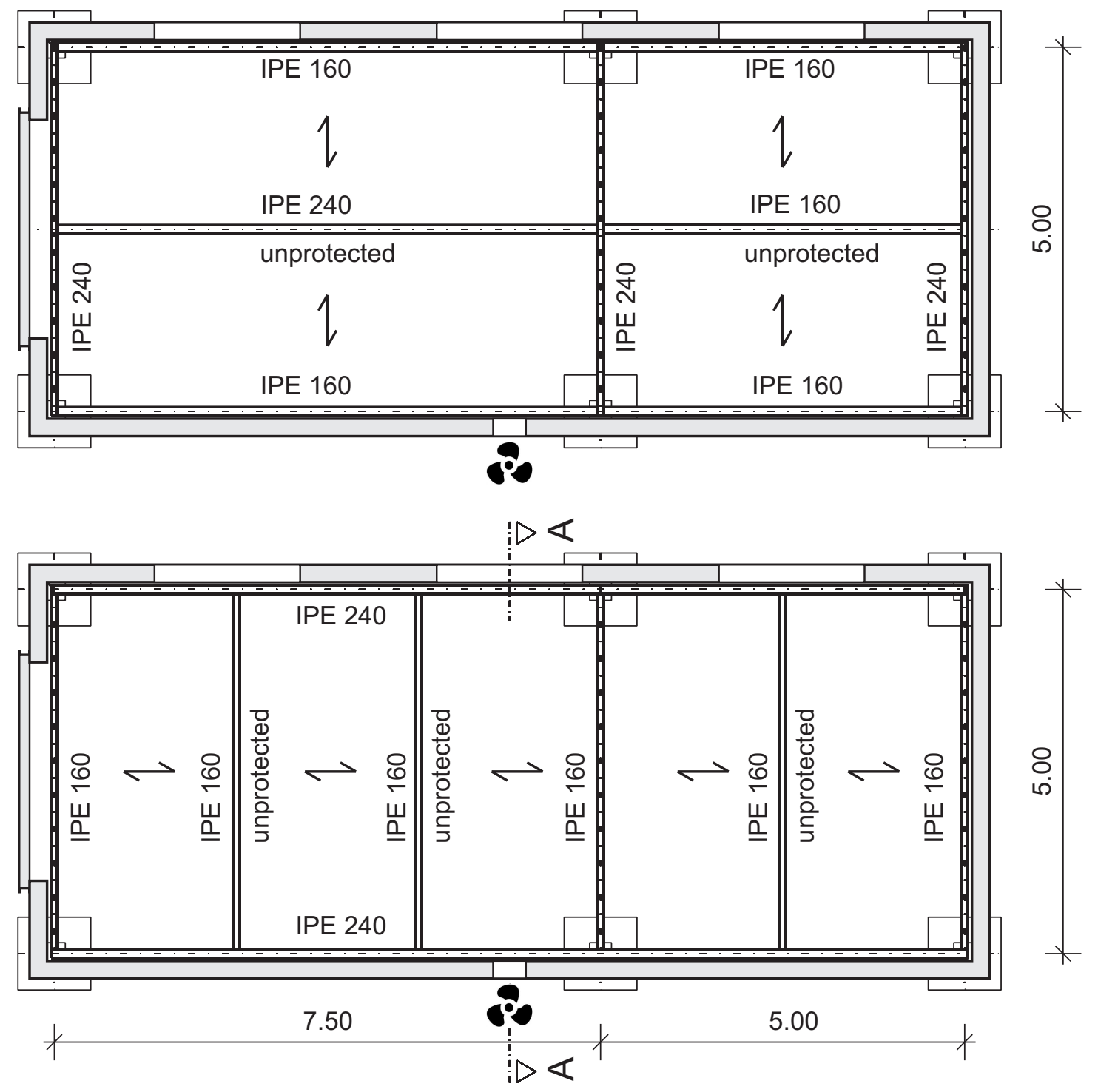

Fig. 3.1: Plan view of test 1 (above) and 2 (below) according to [42]

The top reinforcement of both slabs consisted of a welded mesh Q188 grade S500(A) which provided a reinforcement area of $1.88 \mathrm{~cm}^{2} / \mathrm{m}$ in both directions. It resulted from the design of the hogging moments in the slabs above the secondary beams. The top layer was placed $2.5 \mathrm{~cm}$ from the top surface of the slab. The bottom reinforcement of specimen 1 consisted of bars diameter $8 \mathrm{~mm}$ with an area of $2.5 \mathrm{~cm}^{2} / \mathrm{m}$ in longitudinal direction of the slab span and a transverse reinforcement area of $2.0 \mathrm{~cm}^{2} / \mathrm{m}$. The connections between the elements were bridged with rebars diameter $8 \mathrm{~mm}$, spacing $20 \mathrm{~cm}$. Specimen 2 was set up with a composite slab system Holorib HR51 with a galvanized steel sheeting of $0.75 \mathrm{~mm}$ thickness. The sheeting was continuous over the whole length. No bottom reinforcement was used in this test, also no bars in the troughs, since they are not required for membrane action. U-bars of $8 \mathrm{~mm}$ diameter passing around the shear studs were used along the edges to anchor the reinforcement and to avoid longitudinal splitting according to DIN EN 1994-1-1, 6.6.5.3 [31].

An in-situ concrete C25/30 topping was added which was poured for both specimens on 30th April. 

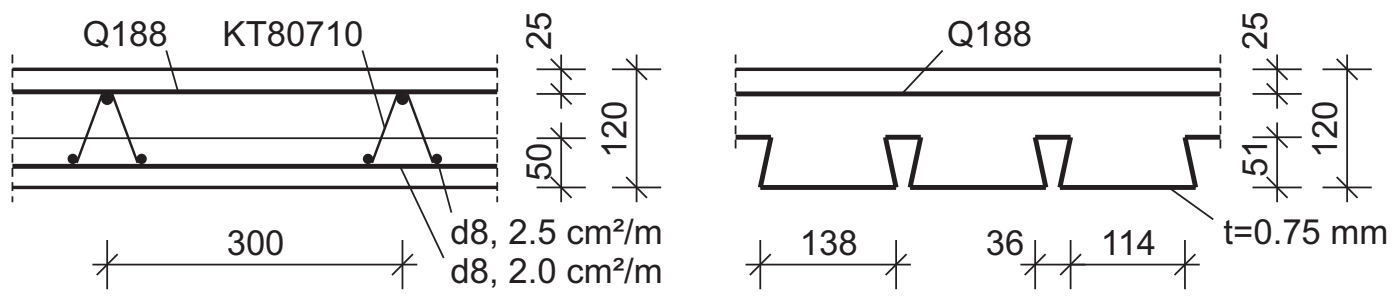

Fig. 3.2: Slab cross-section of first (left) and second (right) Munich test

The actual material properties of the concrete on the day of the tests compared to the values from DIN EN 1992-1-1, Table 3.1 [26] are shown in Table 3.1. The compressive strength was measured on six cylindrical specimens and averaged to its mean value $f_{c m}$. The tensile strength was determined with the splitting tensile strength $f_{c t, s p}$ on three cylinders. The mean value of the axial tensile strength $f_{c t m}$ can be converted approximately with an equation of DIN EN 1992-1-1, 3.1.2 (8) [26]:

$$
f_{c t m}=0.9 f_{c t, s p}
$$

The mean value of the Young's modulus $E_{c m}$ was measured on three cylinders. It can be seen that the actual material properties of the first test compare well with the values of Eurocode 2. They are just slightly higher. The values of the second test are all higher. The reason is that the concrete was already 19 weeks old on the day of the test. This is much more than the age of 28 days that is specified in testing codes to determine the nominal strength.

Table 3.1: Measured concrete material properties in $\left[\mathrm{N} / \mathrm{mm}^{2}\right]$ of Munich tests

\begin{tabular}{|l|c|c|c|c|}
\hline & $f_{c m}$ & $f_{c t, s p}$ & $f_{c t m}$ & $E_{c m}$ \\
\hline C25/30 EC2 & 33.0 & - & 2.6 & 31000 \\
\hline Test 1 & 33.1 & 3.02 & 2.72 & 32881 \\
\hline Test 2 & 39.4 & 3.99 & 3.59 & 36845 \\
\hline
\end{tabular}

The primary and secondary beams in both tests consisted of IPE 160 or IPE 240 sections with steel grade S235. Tensile tests delivered an actual strength of the IPE 160 beams of $f_{y}=315 \mathrm{~N} / \mathrm{mm}^{2}$ and of the IPE 240 beams of $f_{y}=329 \mathrm{~N} / \mathrm{mm}^{2}$. The beams were connected to the slabs with shear studs of diameter $16 \mathrm{~mm}$, length $100 \mathrm{~mm}$. The spacing of the studs varied between $150 \mathrm{~mm}$ and $300 \mathrm{~mm}$ depending on the required shear connection. The connections IPE 240 to IPE 240 were designed with long fin plates and the IPE 160 to IPE 240 connections with end plates. All bolts were type M12, grade 8.8. The unprotected secondary beams were coated with corrosion protection and finishing. The edge and intermediate beams additionally got a layer of intumescent coating in between. The thickness of this layer was specified for a fire resistance category R60. At the first test a water based coating system was used and at the second test a solvent based system.

The slabs were placed on six reinforced concrete columns with a clear height of about $3.0 \mathrm{~m}$ as shown in Figure 3.3. A furnace was constructed around the columns with $24 \mathrm{~cm}$ thick aerated concrete bricks. At the first test the walls were protected by a layer of $12.5 \mathrm{~mm}$ gypsum boards. In one longitudinal wall three openings with a width of $2.0 \mathrm{~m}$ and a height of $1.25 \mathrm{~m}$ were included in order to provide natural ventilation to the fire. In the opposite wall a fan was installed to readjust the ventilation. The openings were dimensioned with the intention that the gas temperature should closely follow the standard fire 
curve. The software OZone [13], [14] was used for designing the ventilation conditions. It became evident in this project that OZone delivered useful results. A gate in one wall was closed shortly before the start of each test.

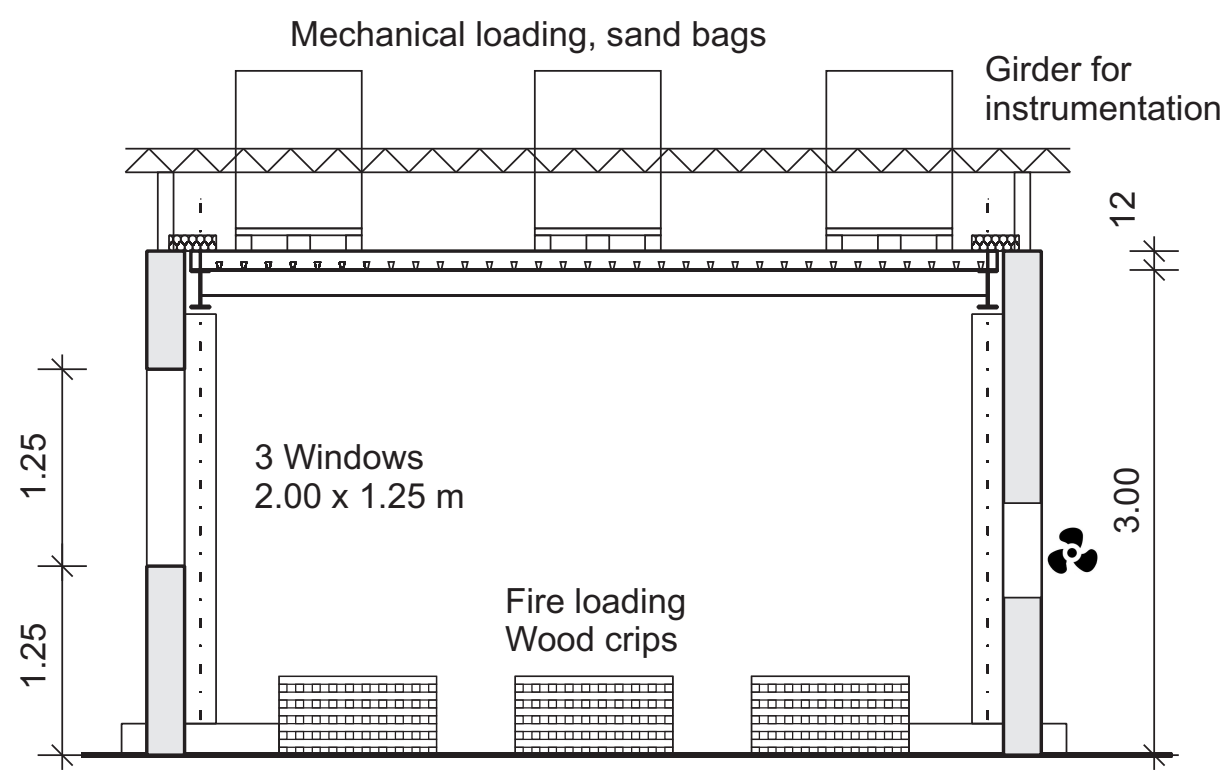

Fig. 3.3: Section A-A of Munich fire tests according to [42]

An additional mechanical load was placed onto the slabs. 15 bags, each filled with $875 \mathrm{~kg}$ sand, were placed evenly to provide a uniform load of $2.1 \mathrm{kN} / \mathrm{m}^{2}$. This value results from an additional dead load of $1.5 \mathrm{kN} / \mathrm{m}^{2}$ and a live load of $2.0 \mathrm{kN} / \mathrm{m}^{2}$ combined for the accidental design situation. In this load combination the quasi-permanent value of the combination factor $\psi_{2}=0.3$ is used as specified in the German national annex of DIN EN 1990 [22]. For the fire load the German national annex of DIN EN 1991-1-2 [25] specifies a fire load density of $584 \mathrm{MJ} / \mathrm{m}^{2}$ for office buildings. With a net calorific value including moisture of spruce timber of $H_{u}=17.3 \mathrm{MJ} / \mathrm{kg}$ according to DIN 18230-3 [18] a required amount of timber of $33.8 \mathrm{~kg} / \mathrm{m}^{2}$ was calculated. The actual fire load was provided by 21 wood cribs inside the furnace. Each crib consisted of 94 spruce timber battens $5 \times 5 \times 100 \mathrm{~cm}^{3}$ which results in a slightly higher timber amount of $35.7 \mathrm{~kg} / \mathrm{m}^{2}$. The reason for the larger amount was that the net calorific value and, hence, the fire load density depends on the moisture of the timber. The energy that is necessary to boil away the water in the material is not available to heat up the furnace. A specific value of moisture could not be guaranteed in the tests since the wood cribs had to be installed earlier and therefore had to stay in the open furnace for several hours. To ensure that the required fire load density was reached, the amount of timber was increased. The moisture of the timber in the first test was measured at $11.2 \%$ and in the second test at $12.0 \%$.

The temperature development in the members was measured by 70 type $\mathrm{K}$ thermocouples in the beams, connections and over the slab depth on several locations. The gas temperature in the furnace was measured at 9 locations in the first test and 8 in the second test. The thermocouple lances were placed about $30 \mathrm{~cm}$ beneath the bottom surface of slab. A total of 20 measuring points for vertical and horizontal deformations were installed. Sketches with the locations of the measuring points will be given in this work when the test data is used. Detailed drawings can be found in the report of the research project [42]. The data was recorded over a period of five hours in order to include the behaviour of the structure in the cooling phase. 


\subsubsection{Results test 1}

After a short ignition phase the temperature time curve of the gas in the first test approximately followed the standard fire curve as shown in Figure 3.4. After 40 minutes the mean temperatures reached a maximum of about $900^{\circ} \mathrm{C}$ before these began to decrease again. The maximum temperatures in the furnace were higher than $1000^{\circ} \mathrm{C}$. Under the larger slab panel the fire developed slightly faster and reached its maximum already after 30 minutes. After 40 minutes the temperature under the smaller panel also reached more than $1000^{\circ} \mathrm{C}$ but under the larger one it had dropped already to $800^{\circ} \mathrm{C}$ at this time, therefore, the average temperature remained lower.
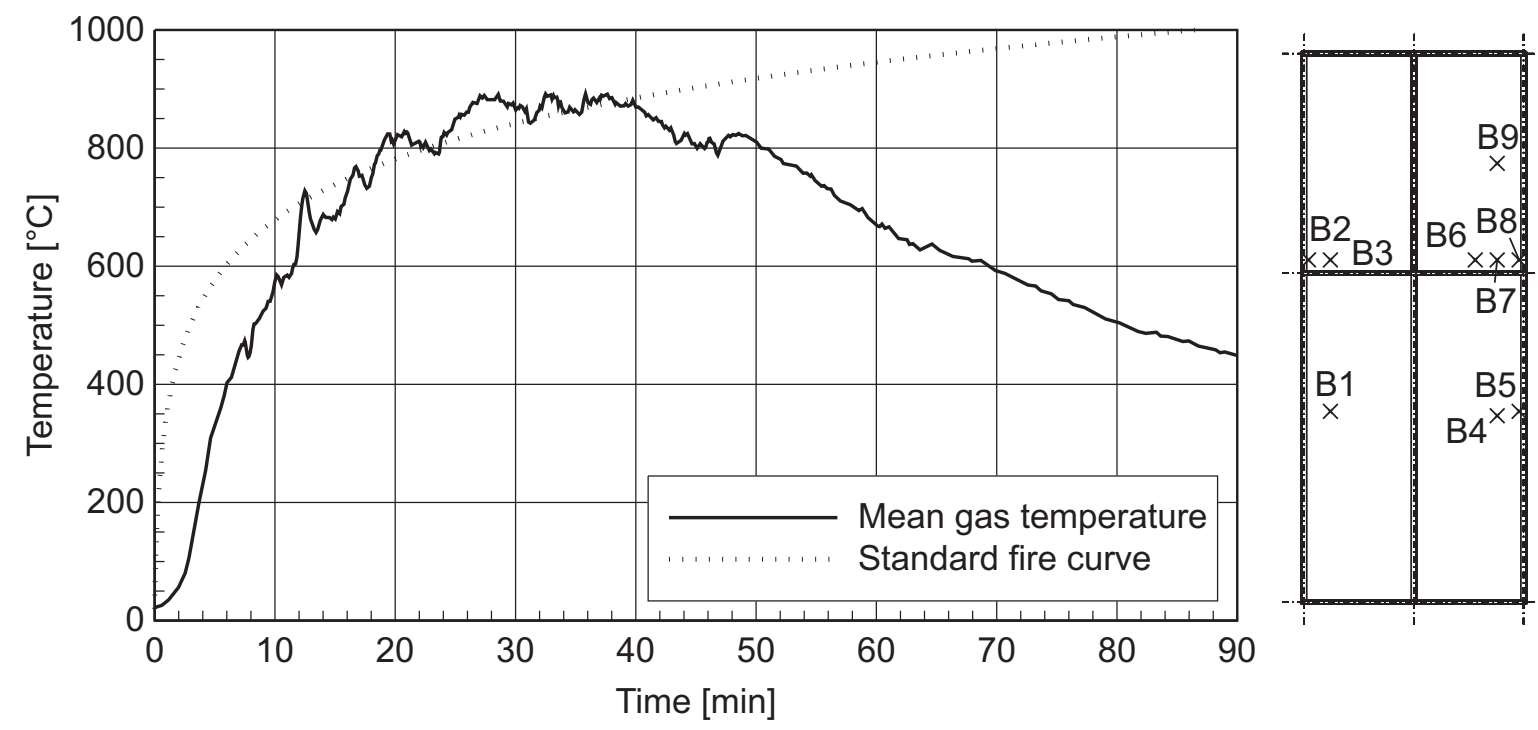

Fig. 3.4: Mean gas temperature of first Munich test according to [42]

In Figure 3.5 the temperature development in the slab is shown. On curve D11a can be seen that the temperature at the bottom surface approximately followed the gas temperature at the beginning. After about 12 minutes the temperature at the bottom reinforcement (D11b) reached $100^{\circ} \mathrm{C}$ and remained constant until all of the uncombined water in the concrete had boiled away. Shortly after this time the
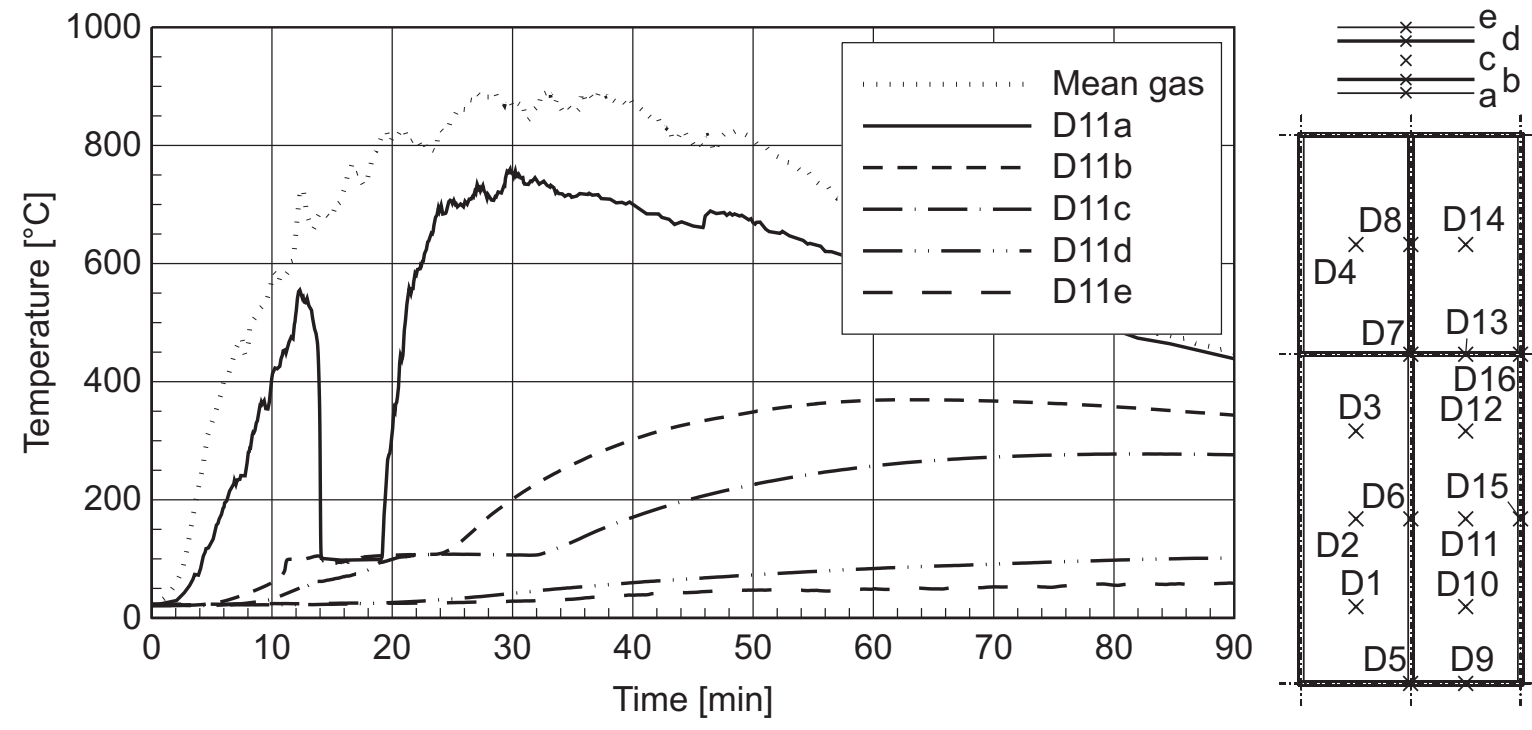

Fig. 3.5: Temperatures in slab of first Munich test according to [42] 
temperature at the bottom surface (D11a) also dropped to $100^{\circ} \mathrm{C}$. The steam streamed from inside the slab to the surface and cooled the surface down. The concrete layer at the surface itself did not contain uncombined water. This can be seen on curve D11a which does not show a temperature plateau when the first time $100^{\circ} \mathrm{C}$ were reached. The top reinforcement (D11d) remained very cold. It barely reached $100^{\circ} \mathrm{C}$ after 90 minutes.

The temperatures in the unprotected secondary beam at the larger slab panel closely followed the gas temperature. This can be seen in Figure 3.6, curves T6a to T6c. The temperatures reached their maximum after 30 minutes like the gas temperature under the larger slab panel as described above. The unprotected secondary beam at the smaller panel reached the maximum temperature after 40 minutes like the gas temperature in that area. The top flange (T6c) remained colder with maximal $700^{\circ} \mathrm{C}$. It had direct contact to the slab and some of the heat was conducted into the concrete. The protected edge beams were sufficiently protected and the temperatures remained below $300^{\circ} \mathrm{C}$ which can be seen on curves T10a to T10c. Again the temperatures in the top flange (T10c) were lower than in the remaining section.
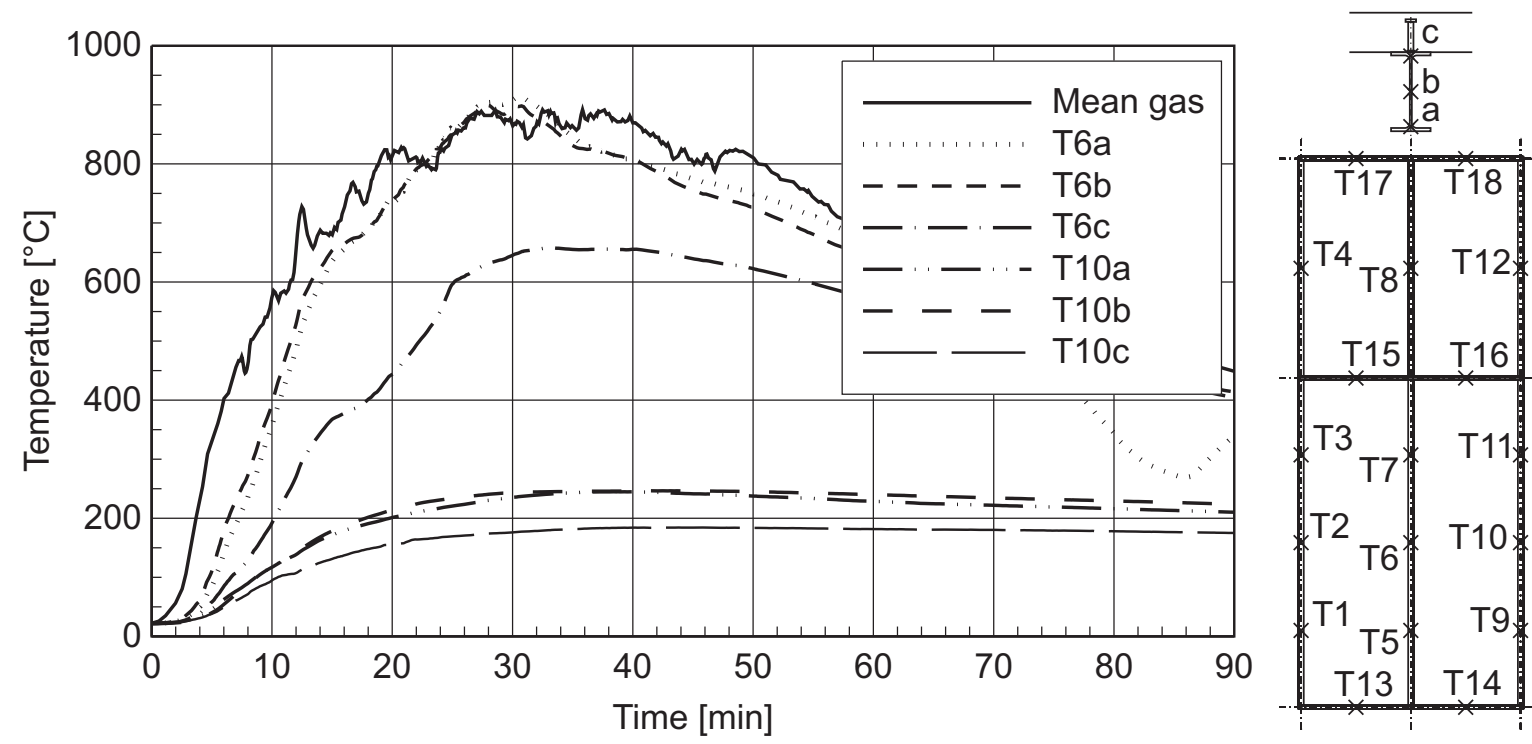

Fig. 3.6: Temperatures in protected edge beam and unprotected secondary beam of first Munich test according to [42]

At the intermediate beam between the two slab panels the intumescent coating partly detached from the bottom flange after expansion. This process started after approximately 16 minutes as shown in Figure 3.7. The temperatures in curve T16a suddenly rose much faster from this moment on, therefore, the bottom flange almost reached the same temperature level as the surrounding hot gas. At the web the protective foam was held by the bottom flange and could not detach. Temperatures remained lower in the remaining parts which can be seen on curves $\mathrm{T} 16 \mathrm{~b}$ and $\mathrm{T} 16 \mathrm{c}$, however, these still reached almost $500^{\circ} \mathrm{C}$ since the beam was exposed to the fire from three sides and a large amount of energy was conducted from the bottom flange to the remaining section. The unsteadiness of curve T16b and T16c can be explained by non-uniform foaming of the intumescent coating.

In Figure 3.8 the vertical deformations along a section in longitudinal direction in the middle of the slab are shown. It can be seen that the larger panel (V6) reached a maximum deformation of about $260 \mathrm{~mm}$ after 60 minutes and the smaller panel $200 \mathrm{~mm}$ ten minutes later. The reason for the different times is again that the gas temperature reached its maximum 10 minutes later under the smaller panel. Not shown in Figure [42] is that the longer edge beam at the side of the furnace with the windows 

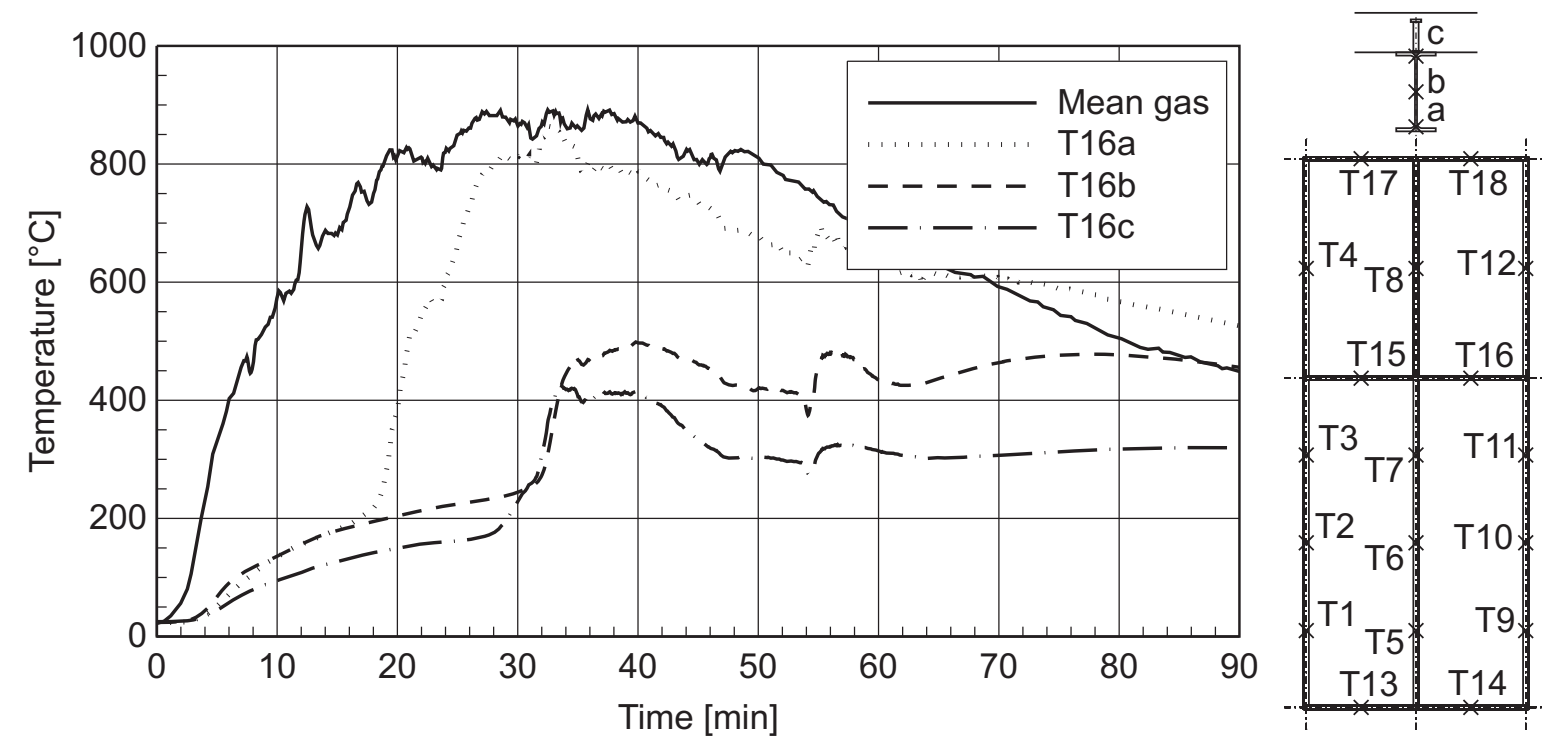

Fig. 3.7: Temperatures in protected intermediate beam of first Munich test according to [42]

(V2) had a maximum deformation of $110 \mathrm{~mm}$. The beam on the opposite side (V11) reached $140 \mathrm{~mm}$. After complete cooling the deformations reduced by approximately $50 \%$. The measured horizontal displacements did not deliver reasonable results in the first test.
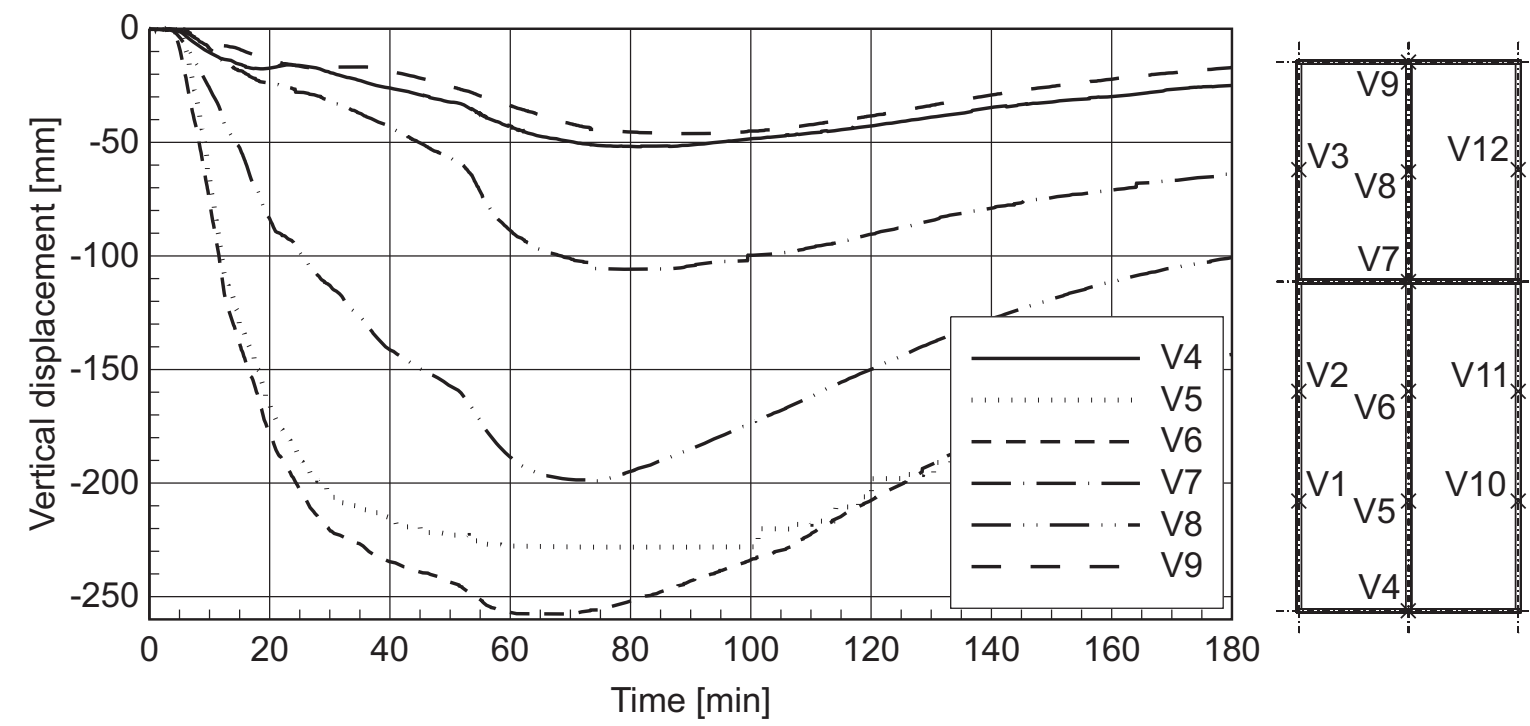

Fig. 3.8: Vertical displacements of first Munich test according to [42]

The construction did not collapse during the whole test period. The load bearing criterion " $R$ " was satisfied the whole time. After about 19 minutes gaping cracks occurred nearby the intermediate beam as shown in Figure 3.9. Smoke streamed through crack 1 to the upper side of the slab. Therefore, the slab failed in terms of the integrity criterion "E" and at this location also in terms of the thermal insulation criterion "I".

A top view of the whole crack pattern is shown in Figure 3.9. The cracks ran across the whole slab width. These occurred, both, directly above the intermediate beam (crack 2 and 3) and about $30 \mathrm{~cm}$ to one side, in the smaller slab panel (crack 1 and 4). At the window side, the cracks above the beam (cracks 2) remained small. Crack 1 opened several centimetres over the whole slab depth as shown in Figure 3.10. In contrast, on the opposite side of the slab the crack above the intermediate beam (crack 3) 


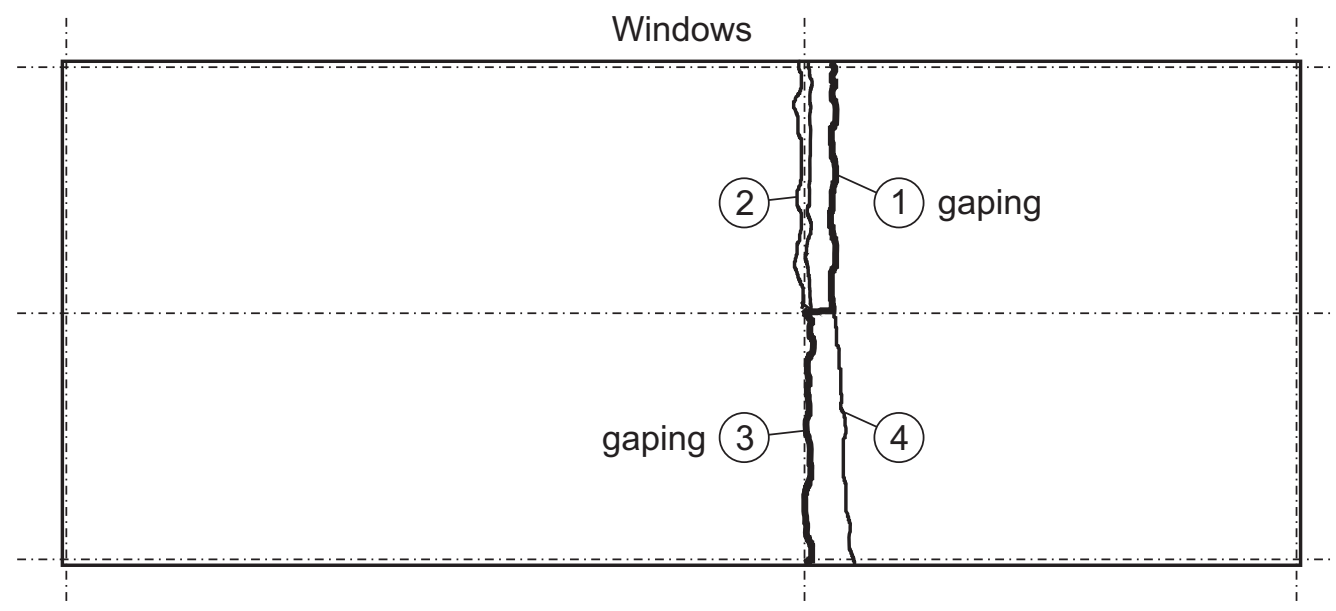

Fig. 3.9: Crack pattern of first Munich test according to [42]

opened and the crack inside of the slab panel (crack 4) remained small. Crack 4 was not visible on the bottom surface of the slab. The cracks inside of the slab panel (crack 1 and 4) ran along the first lattice girder. In the gaping crack 1 most of the bottom reinforcement broke by shear failure. The top layer broke entirely. The ends of the bars in the crack were constricted which suggests that the reinforcement failed due to a tensile force, not shear.

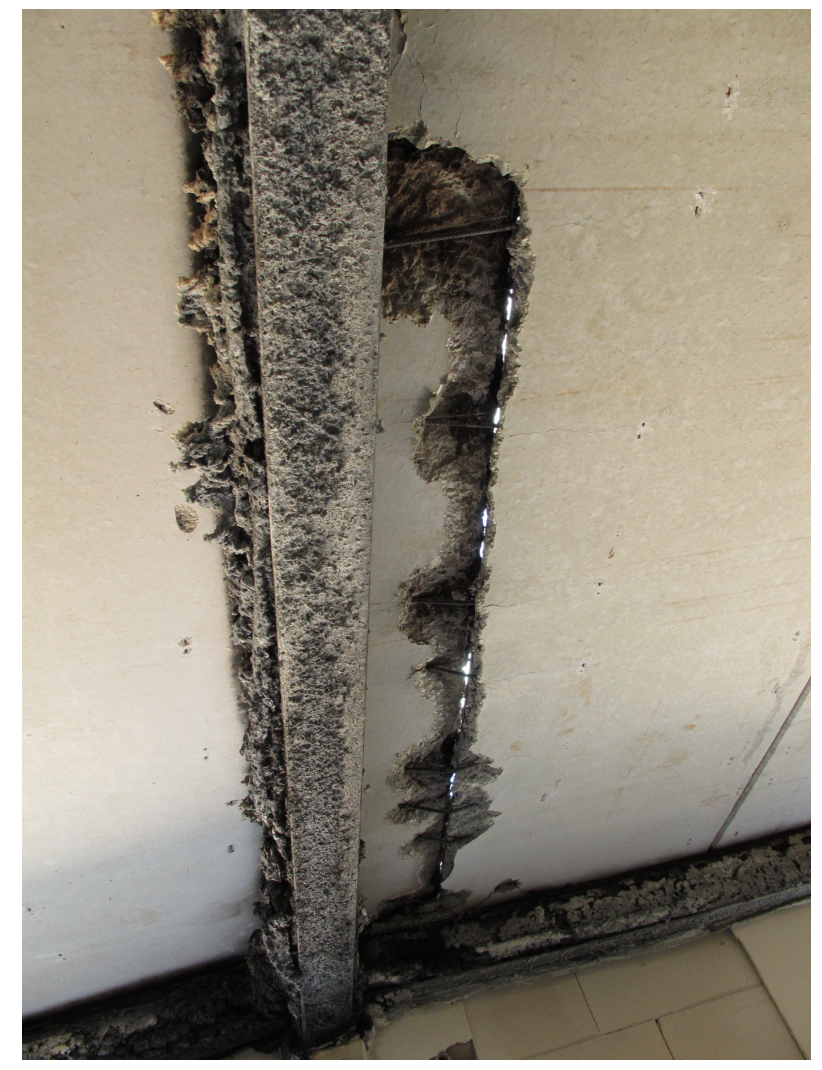

Fig. 3.10: View from below at gaping crack at first Munich test according to [42]

In the report of the DASt project [42] the reason for the gaping cracks is explained as follows and is confirmed by further research work on this issue. Large hogging moments and tensile forces occurred above the intermediate beam due to mechanical loads and restraint thermal elongation. These forces caused a first crack in the concrete as soon as the tensile strength was reached. On the one hand, the 
concrete cracked at the location with the highest bending moment directly above the intermediate beam. On the other hand, it cracked at the first lattice girder which provided a weakening in the structure as shown in Figure 3.11. The tensile forces were larger than the forces that could be carried by the top reinforcement. No further cracks could develop due to the small amount of reinforcement and the first cracks started gaping. The theoretical background of this issue will be explained in detail in Section 5.2.3. Which of the cracks opened or remained closed was caused by statistical strength distribution. The whole deformation had to be carried out by the first cracks. Therefore, the crack widths became very large. The top reinforcement reached its ultimate strain in the crack and ruptured. The crack directly above the intermediate beam (crack 3) could gape without causing integrity failure since the smoke was hindered from streaming through the crack by the steel beam. At crack 1 the gaping caused a secondary failure mode in the slab. Since the reinforcement was ruptured, the shear transferring mechanisms such as tensile crack bridging, dowel action and friction due to aggregate interlock were not able to act any longer and the slab failed due to shear forces.

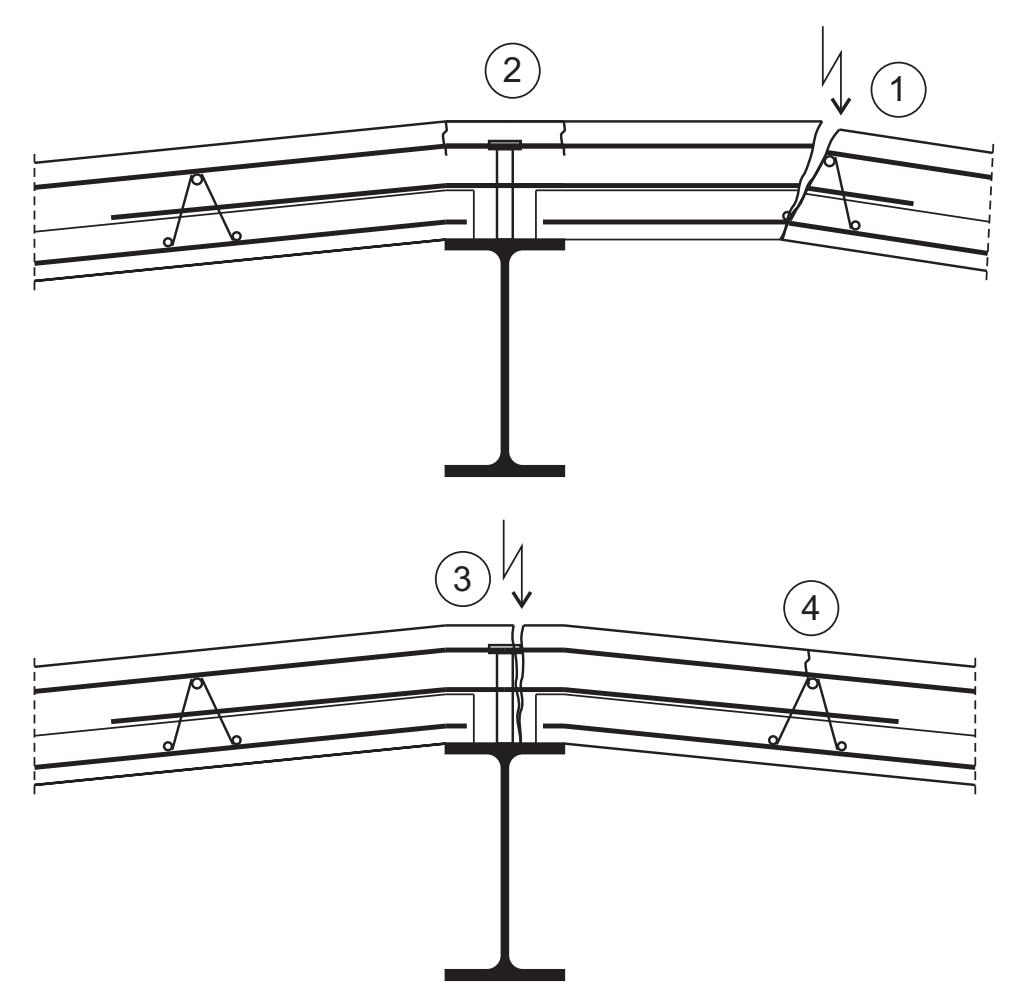

Fig. 3.11: Section with cracks of first Munich test according to [42]

This kind of secondary failure is well-known at ambient temperature for punching shear at flat slabs. In the field of membrane action it did not attract any interest so far. In all currently available simple calculation models, it is assumed that the reinforcement around the boundaries of a slab panel indeed ruptures and therefore the consideration of separated panels is justified, but it is also assumed that the rupture does not cause failure. This has to be revised after the latest test results. One of the main objectives of this work is therefore to provide a method for designing a required reinforcement amount above intermediate beams in order to prevent failure at this location. 


\subsubsection{Results test 2}

In the second test the gas temperatures reached their maximum of about $900^{\circ} \mathrm{C}$ after 40 minutes as shown in Figure 3.12. The temperature-time curve of the gas in the furnace barely reached the standard fire curve. The reason was over-ventilation by the fan. In order to accelerate the temperature increase, the ventilation rate was very high at the beginning of the test. The contrary effect was reached, however, that more hot gas was pressed out through the windows, more cold air was led into the furnace and, as a result, the temperatures increased slower. In future tests it possibly makes sense to use smaller windows to be better able to regulate the ventilation by the fan. The temperature distribution in the furnace was more homogeneous in this test. No appreciable differences could be noticed between the temperatures at different locations in the furnace.

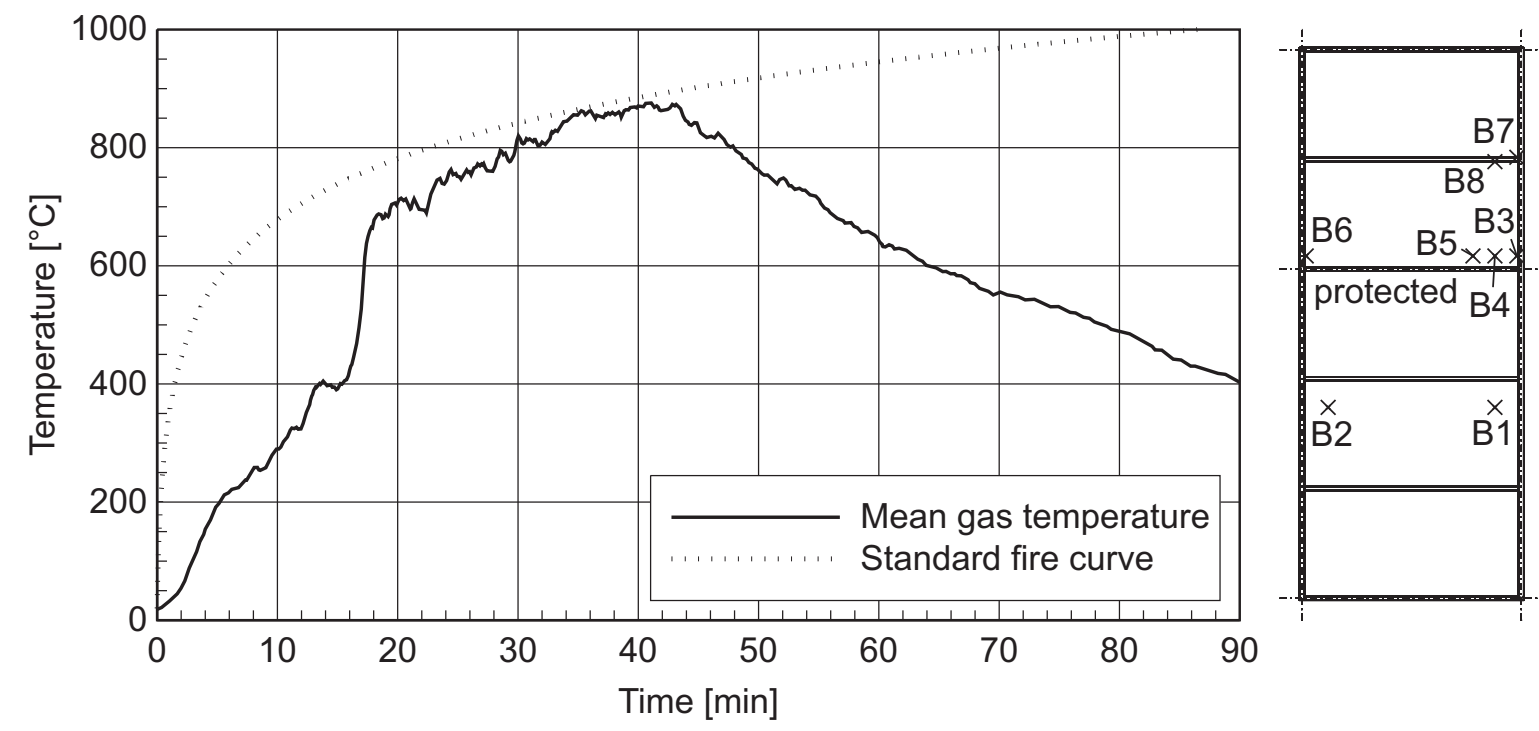

Fig. 3.12: Mean gas temperature of second Munich test according to [42]

The temperature distribution in the slab at measuring point D6 is shown in Figure 3.13. It can be seen that the temperature in the top flange (e) of the steel sheeting remained at less than $350^{\circ} \mathrm{C}$ considerably colder than the bottom flange (a) which was directly exposed to the fire and reached more than $700^{\circ} \mathrm{C}$. A temperature plateau of $100^{\circ} \mathrm{C}$ for approximately 20 minutes can be seen in the trough (b) and at the reinforcement above the top flange (f). No plateau can be found for the reinforcement above the trough (c). There the boiling temperature was reached later when the whole uncombined water was already evaporated. The reinforcement remained rather cold with temperatures less than $150^{\circ} \mathrm{C}$.

The temperature development in the steel beams is shown in Figure 3.14. It can be seen that the edge beams remained relatively cold. The bottom flange reached barely $350^{\circ} \mathrm{C}$ after 60 minutes. The web and the top flange of the protected intermediate beam reached almost $500^{\circ} \mathrm{C}$. The thermocouple at the bottom flange fell out. The intumescent coating also detached partly from the bottom flange but less severely than in the first test. The bottom flange presumably reached $600^{\circ}$ to $700^{\circ} \mathrm{C}$ by comparing the data of both tests. The temperatures in the unprotected beams again closely followed the gas temperature and the top flange remained slightly colder than the remaining section. It is noticeable that the temperatures in the protected beams reached their maximum after the gas temperature already started to decrease. In later stages of the test the beam temperatures were even higher than the gas temperatures. The reasons are that, on the one hand, heat was stored in the beams and, on the other hand, heat was conducted from 


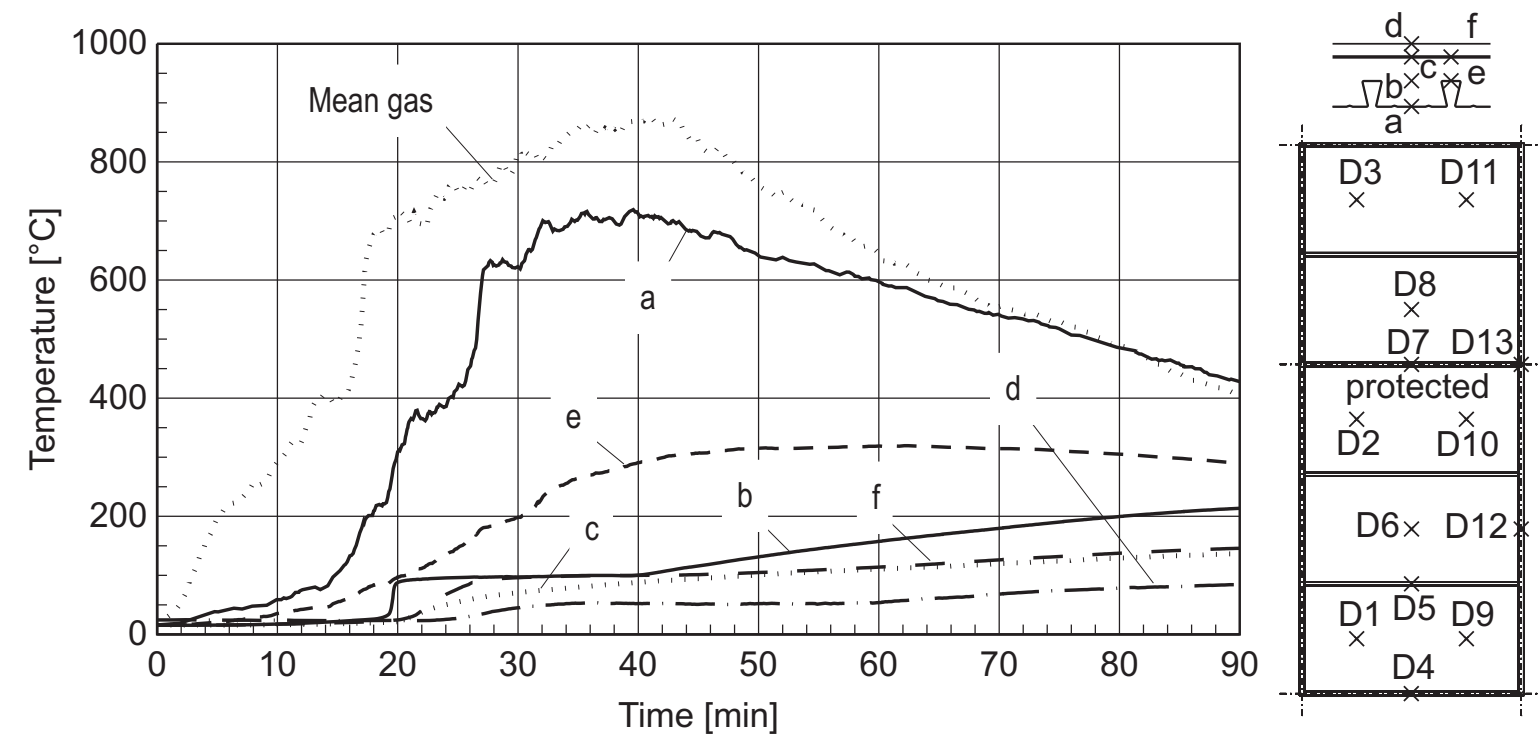

Fig. 3.13: Temperatures in slab, measuring point D6, of second Munich test according to [42]

the slab to the beams when the gas in the furnace already cooled down. The foam of the intumescent coating acted as an insulation, in this case hindering heat flow from the beams to the surrounding gas.
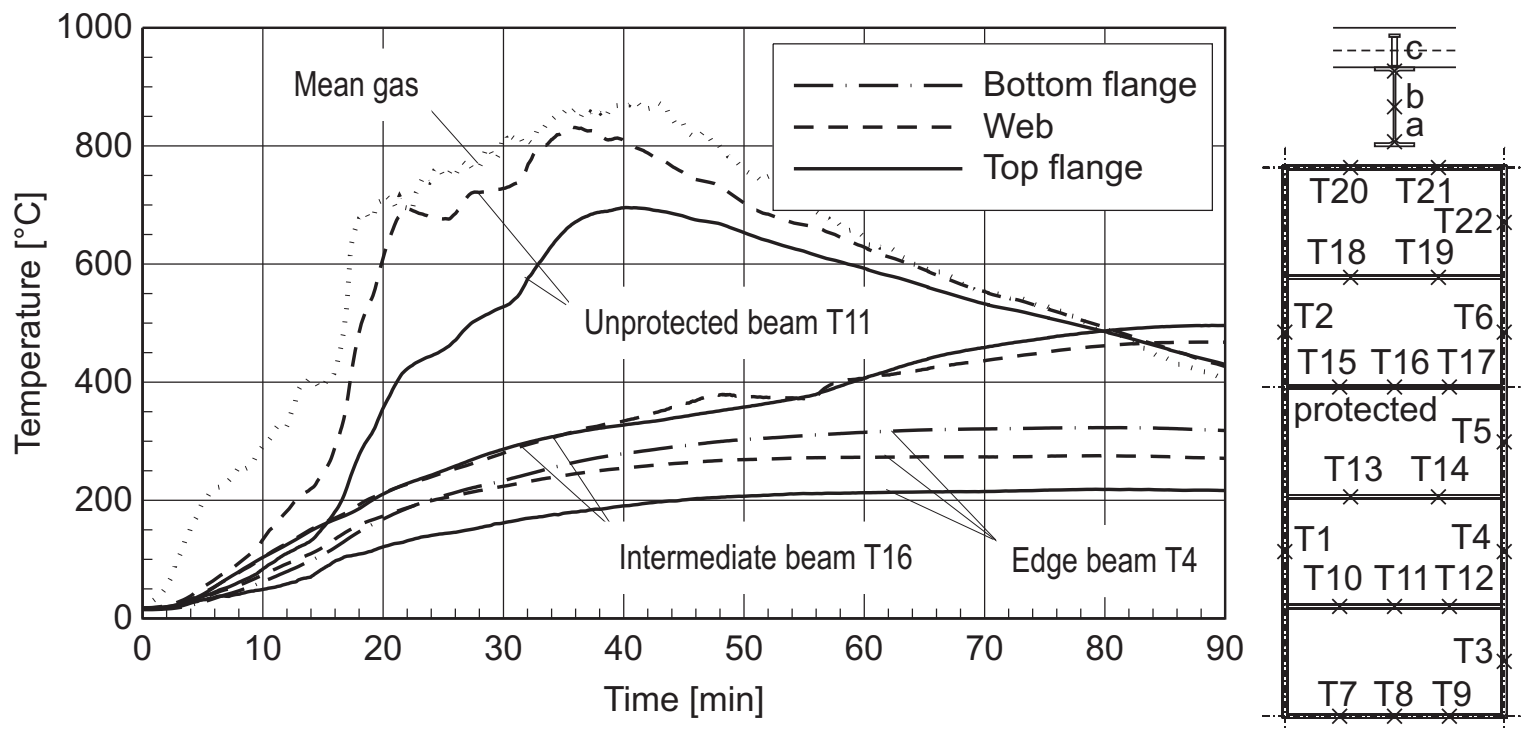

Fig. 3.14: Temperatures in beams of second Munich test according to [42]

The larger slab panel reached a maximum vertical displacement of about $255 \mathrm{~mm}$ after 60 minutes which can be seen from curve V6 in Figure 3.15. The smaller panel (V10) reached a maximum of $190 \mathrm{~mm}$. In the cooling phase after about 150 minutes the intermediate beam (V8) had the same deformation as the smaller slab panel (V10). After the cooling phase the deformations reduced by approximately $50 \%$ as in the first test. Not shown in Figure 3.15 is that the maximum deformation of the edge beams of the larger panel (V4) reached $65 \mathrm{~mm}$ and the smaller panel (V11) $20 \mathrm{~mm}$.

After redesigning the measuring system for the horizontal displacements more reasonable results were delivered than in the first test. The data is shown in Figure 3.16. The results should be used carefully nevertheless. The values show the relative displacement between the slab and the furnace wall since the measuring devices were fixed on the walls. Possible wall deformations may have affected the measuring. 


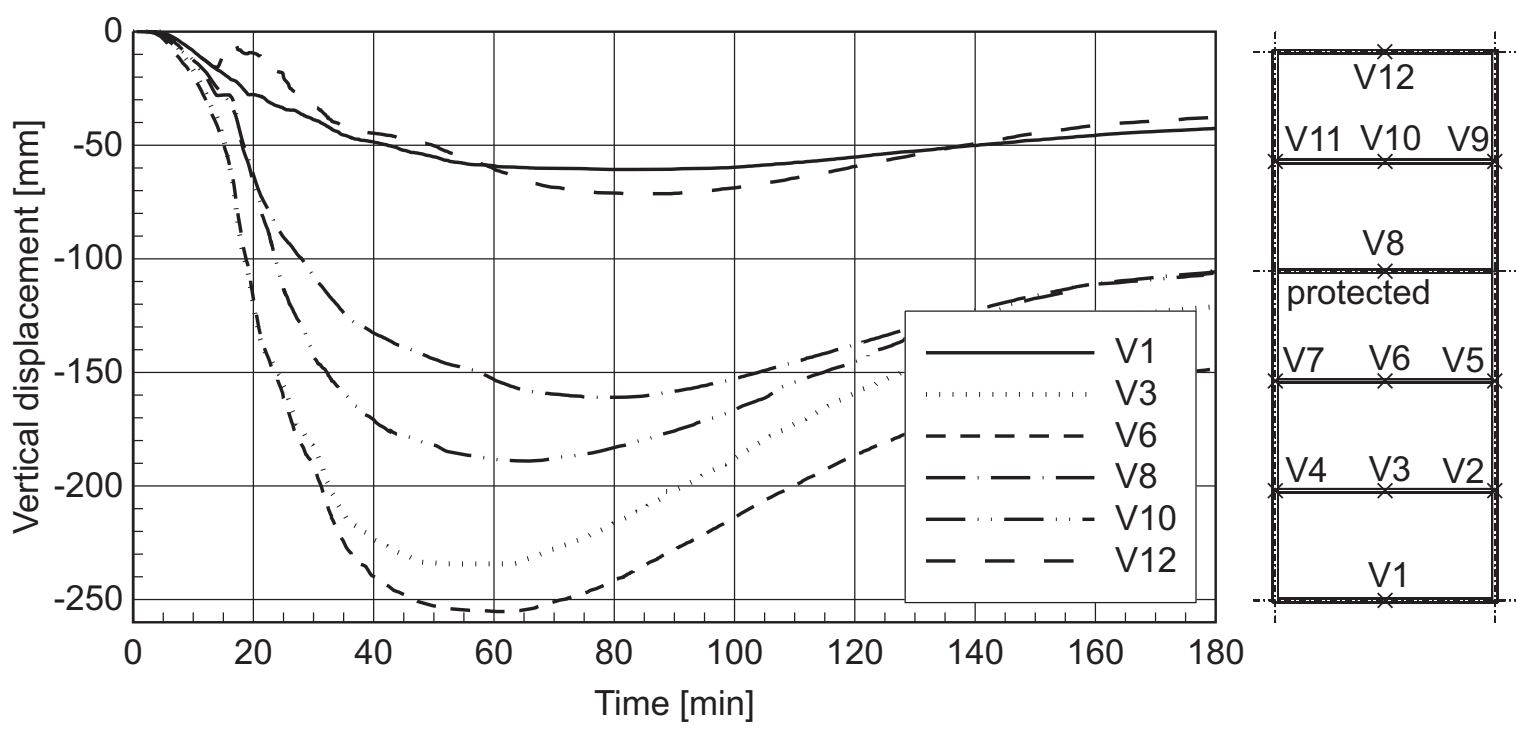

Fig. 3.15: Vertical displacements of second Munich test according to [42]

For future tests a detached frame for the measuring system is recommended which is not connected to the specimen or furnace and is protected against heating to avoid thermal deformation of the frame.
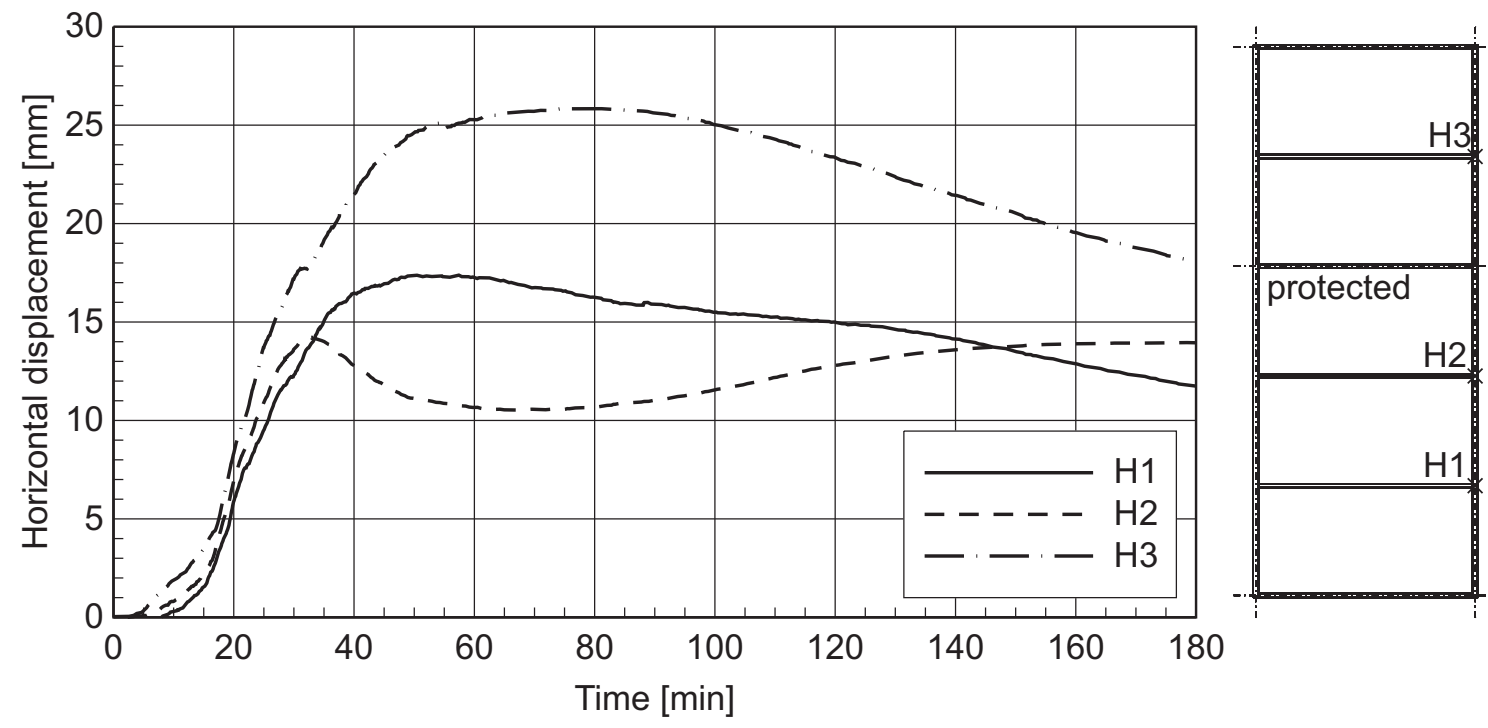

Fig. 3.16: Horizontal displacements of second Munich test according to [42]

In this test no unexpected incidents happened. Above the intermediate beam a single crack occurred across the whole slab after about 20 minutes. This crack was expected and did not lead to any failure although the crack width was several millimetres. A thermocouple on the top surface of the slab directly at the crack recorded a maximum temperature of $135^{\circ} \mathrm{C}$. This value is lower than the specified maximum temperature rise at single locations of $180 \mathrm{~K}$ for the criterion "I" according to DIN EN 1994-1-2, 2.1 .2 [32]. No smoke or flames could be seen coming through the crack, therefore, all three criteria "REI" were satisfied for the whole test. A void between the steel sheeting and the concrete was detected after the test. This indicates that the steel sheeting detached from the concrete during the test, but it did not fall down. The zinc layer on the surface of the steel sheeting melted during the test.

Although no failure occurred, it cannot be generalised that failure can be excluded by using composite slabs. It was fortuitous that the crack in the test occurred exactly above the intermediate beam and did not 
run into the slab panel. If there had been a weakening in the slab at any location nearby the intermediate beam, the crack would have arisen there. In real structures, there are many possibilities of how a slab could be weakened locally, for example through block-outs or non-uniform compacting of the concrete. The crack width remained relatively small since the intermediate beam suffered large deformations and so the rotations remained small in the slab above. The reinforcement in the crack did not rupture due to the small crack width. If the intermediate beam had been stiffer however, the crack would have opened wider, the reinforcement possibly would have ruptured and failure could have occurred. The orientation of the steel sheeting can also influence the location of possible cracks as shown in Figure 3.17. If the ribs of the sheeting run parallel to the intermediate beam, the slab will not break directly above the beam at the location of the largest bending moment but a few centimetres aside where the cross-section is much weaker. In this case the beam cannot hold the slab ends at the same level and cannot hinder the fire to go through the crack. Even if a slab does not collapse when large cracks arise at the intermediate beams, integrity failure can occur and the fire can spread to the storey above the slab. Measures therefore have to be taken in order to avoid gaping cracks above intermediate beams, also if composite slabs are used.

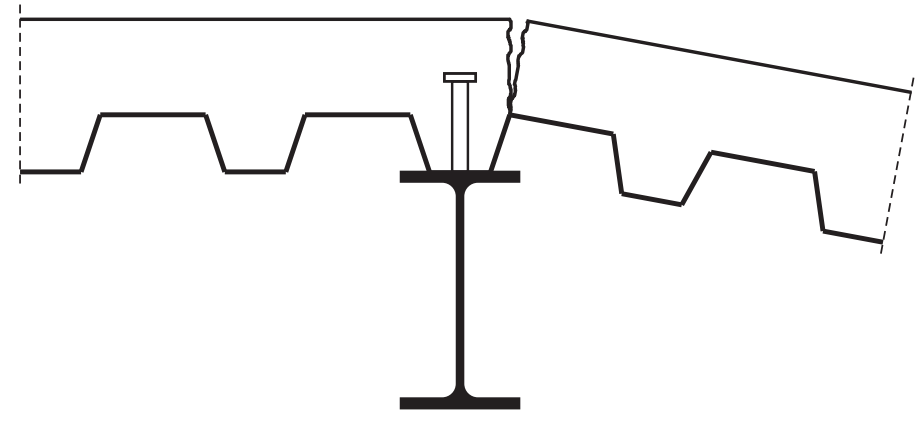

Fig. 3.17: Possible crack location, steel sheeting parallel to intermediate beam

\subsection{FRACOF test}

On 16th January 2008 a full-scale fire test on membrane action was performed at the laboratory of the Efectis fire testing company in Metz / France. This test was part of the FRACOF project which aimed to distribute the knowledge of membrane action outside of Great Britain in Europe. Main project partners were the French and British steel construction associations CTICM and SCI as well as the steel company ArcelorMittal. The main differences to the British tests conducted so far were the use of European steel sections and testing the slab with the standard fire. The results of the test are used in this work for two reasons. On the one hand, the used Cofraplus 60 slab system is commonly used in Germany and open trough profiles should be included in this work. On the other hand, slabs have to be tested or designed with the standard fire curve according to fire protection regulations in Germany. A detailed description of the test can be found in [49].

\subsubsection{Test arrangement}

A single composite slab panel in scale 1:1 was tested with a longer span of $8.735 \mathrm{~m}$, a shorter span of $6.660 \mathrm{~m}$ and a total depth of $15.5 \mathrm{~cm}$. A plan view of the specimen is shown in Figure 3.18 and the crosssection of the slab in Figure 3.19. Two primary beams bridged the short span which consisted of IPE 400 hot-rolled sections, steel grade S355. Four secondary beams ran in longitudinal direction and consisted 


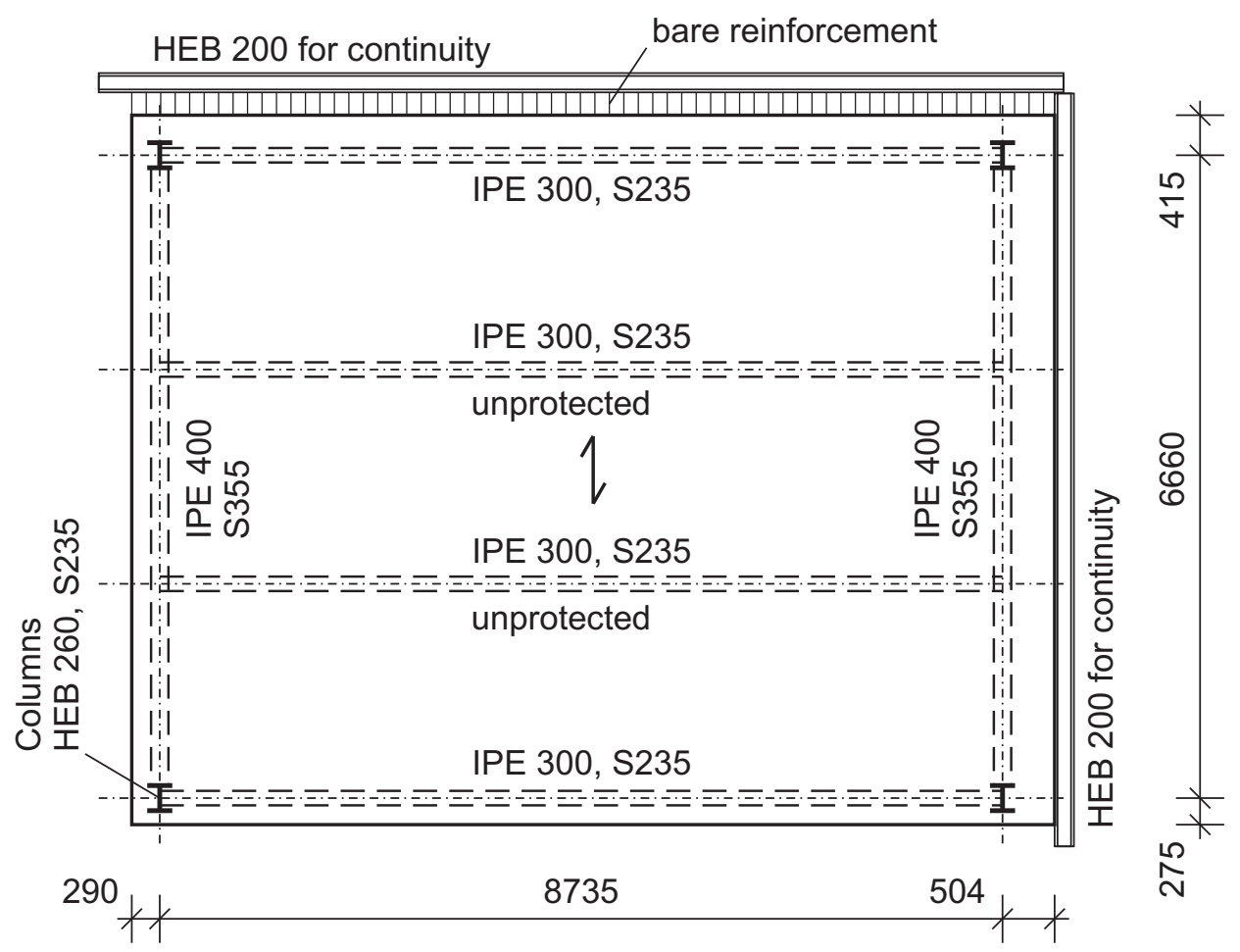

Fig. 3.18: Plan view of FRACOF test on the basis of [49]

of IPE 300 sections in S235. The beams were connected to short steel columns at all four corners which provided a clear height below the slab of $80 \mathrm{~cm}$. The primary beams were connected to the columns with end plates. Double angle connections were used for the secondary beams. The edge beams, connections and columns were protected against heating by two layers of $25 \mathrm{~mm}$ mineral fibre blankets. Such blankets are often used in fire tests due to their flexibility. For real structures these are mostly too expensive. The two secondary beams in the centre of the slab were unprotected. Headed shear studs diameter $19 \mathrm{~mm}$ with a length of $125 \mathrm{~mm}$ were used with a spacing of $207 \mathrm{~mm}$ at the secondary beams and $100 \mathrm{~mm}$ at the primary beams. A galvanised Cofraplus 60 profiled steel sheeting with a thickness of $0.75 \mathrm{~mm}$ spanned in transversal direction of the slab panel. A top reinforcement was placed $50 \mathrm{~mm}$ below the top surface of the slab. It consisted of a mesh of $7 \mathrm{~mm}$ diameter bars, at $150 \mathrm{~mm}$ spacing with a steel grade $\mathrm{S} 500$. This mesh provided a reinforcement area of $2.57 \mathrm{~cm}^{2} / \mathrm{m}$ in both directions. It is not described in the report

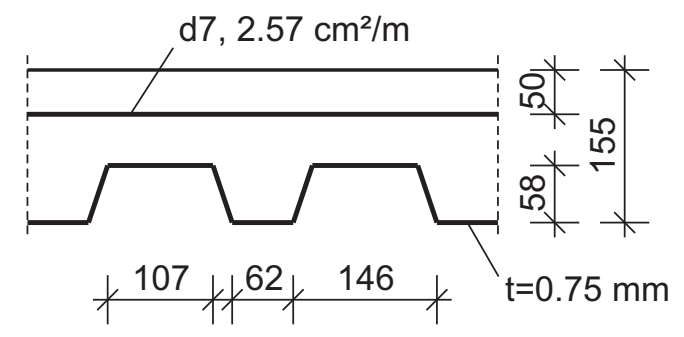

Fig. 3.19: Slab cross-section of FRACOF test

[49] whether the mesh was welded or bound. The only detail given was that a joint of the reinforcement at the slab centre in longitudinal direction was welded. A bottom reinforcement was not used. Additional $10 \mathrm{~mm}$ diameter bars were placed around the columns. The reinforcement mesh was welded to steel beams at two edges of the slab. This should simulate an interaction with adjacent slab panels. On the longitudinal edge the reinforcement was coming out of the slab and was not covered by concrete for 
several centimetres before it was welded to the beam. This part of the reinforcement buckled during the test and had therefore not the expected effect. A normal weight concrete class C30/37 was used which had a measured compressive strength of $f_{c m}=36.7 \mathrm{~N} / \mathrm{mm}^{2}$. The report [49] does not mention whether this value was measured at the day of the test or after 28 days according to the testing codes. The secondary beams offered an actual strength of $f_{y}=311 \mathrm{~N} / \mathrm{mm}^{2}$, the primary beams $f_{y}=423 \mathrm{~N} / \mathrm{mm}^{2}$ and the reinforcement $f_{y}=594 \mathrm{~N} / \mathrm{mm}^{2}$.

The specimen was placed on top of an existing furnace, the slab forming the ceiling of the furnace. 15 sandbags were placed evenly on the slab. Each weighed $1500 \mathrm{~kg}$ which resulted in an additional mechanical load of $3.87 \mathrm{kN} / \mathrm{m}^{2}$. The gas temperature in the furnace followed the standard fire curve and was controlled by plate thermocouples. The furnace was heated by burners in the walls. It is not documented in the report [49] whether these were gas or oil fuelled. The temperature distribution in the members was measured by about 170 thermocouples. The vertical displacement was measured at seven locations and the horizontal displacement at two locations. The heating phase lasted slightly more than 120 minutes. The test was stopped after 105 minutes as a large crack at the slab centre occurred.

\subsubsection{Results}

The gas temperature in the furnace closely followed the standard fire curve. The temperature distribution in the slab is shown in Figure 3.20. Measuring points A and B in the steel sheeting failed very early in the test and were not recorded. This data would have been interesting for numerical simulations. All curves show a temperature plateau at $100^{\circ} \mathrm{C}$ when the uncombined water boiled away as at the Munich tests. The concrete temperature in the troughs reached almost $700^{\circ} \mathrm{C}$ which can be seen from curve $\mathrm{C}$. The temperature in the reinforcement above the troughs $(\mathrm{E})$ remained about $100^{\circ} \mathrm{C}$ lower than above the top flange of the sheeting (F). The average temperature in the reinforcement reached about $300^{\circ} \mathrm{C}$. Not shown in Figure 3.20 is the temperature development at the top surface of the slab. This reached between $75^{\circ}$ and $100^{\circ} \mathrm{C}$ after 105 minutes and between $80^{\circ}$ and $110^{\circ} \mathrm{C}$ after 120 minutes.

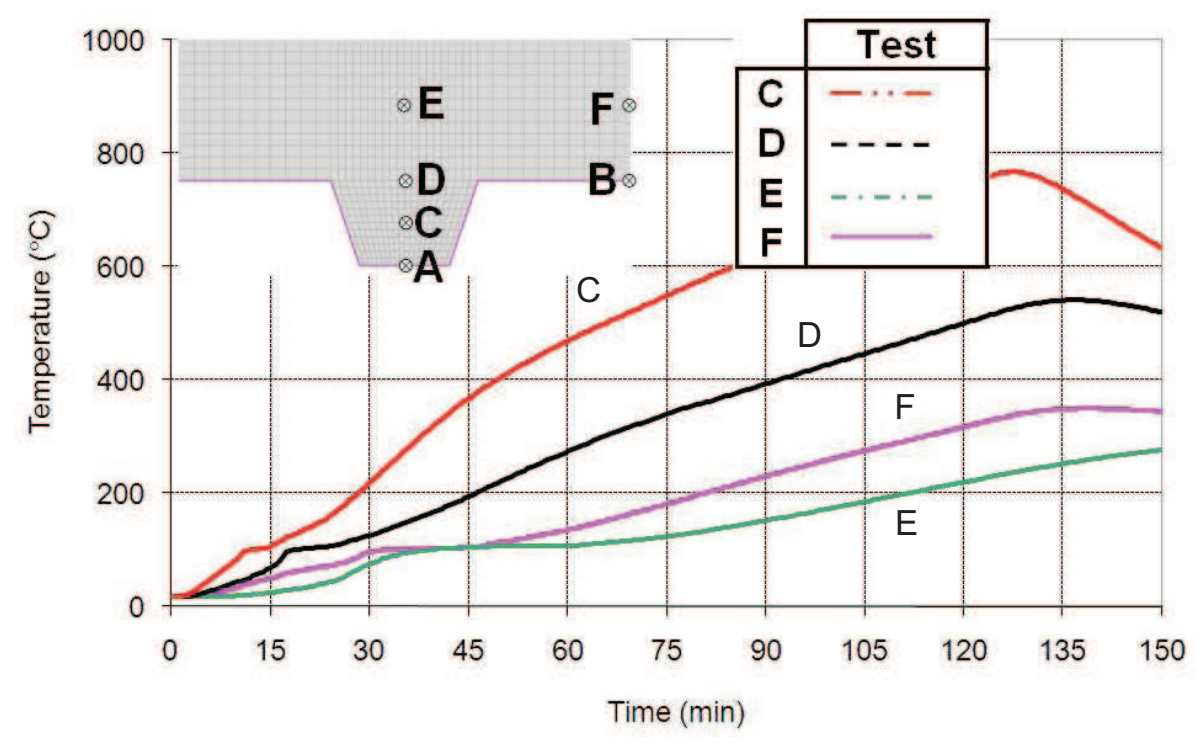

Fig. 3.20: Temperatures in slab of FRACOF test according to [49]

Figure 3.21 shows the temperature development of a protected edge beam. It unclear from the report [49] whether the shown curves belong to a primary or secondary beam. Since the primary beams were 
more compact, the temperatures were probably lower than in the secondary beams. The edge beams remained relatively cold with a maximum temperature of the bottom flange of less than $300^{\circ} \mathrm{C}$. In real structures these will heat up considerably more with major effects on the load bearing behaviour. Not shown in Figure 3.21 is the temperature development in the unprotected beams. These closely followed the gas temperature as at the Munich tests and reached a maximum of slightly more than $1000^{\circ} \mathrm{C}$ until the test was stopped. The top flange again remained approximately $50^{\circ} \mathrm{C}$ cooler than the remaining section.

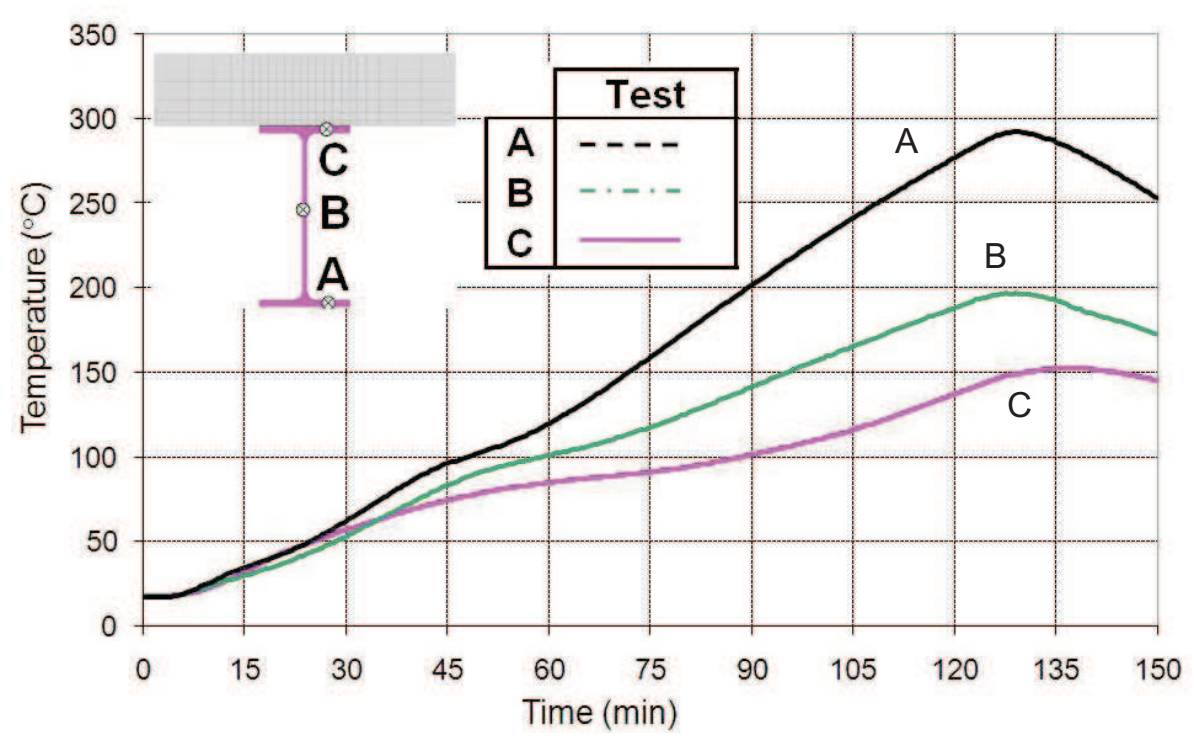

Fig. 3.21: Temperatures in protected edge beam of FRACOF test according to [49]

The development of vertical displacements is shown in Figure 3.22. The centre of the slab reached its maximum displacement of about $470 \mathrm{~mm}$ after 135 minutes, after the heating of the furnace was already stopped. The edge beams experienced relatively small deformations since their temperatures were not high.

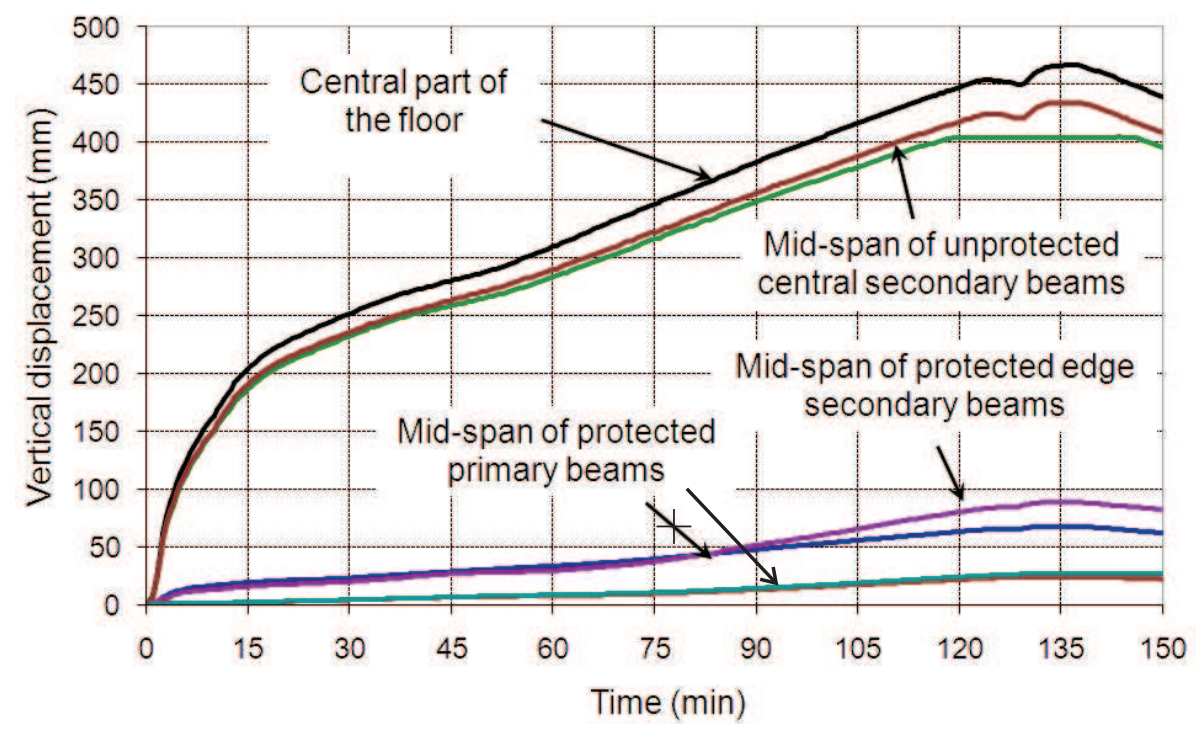

Fig. 3.22: Vertical deformations of FRACOF test according to [49]

Cracks have been observed on the top of the slab running diagonally across all corners. These cracks 
arose very early in the test but did not cause any failure. After 105 minutes a large crack at the centre of the slab in a transversal direction occurred. The test was stopped shortly after since this crack spread over the whole slab depth, opened widely and the hot gas from inside the furnace streamed up to the top of the slab. The structure did not collapse and the criterion " $R$ " was satisfied for more than two hours. Due to the large gaping crack, the slab failed in terms of the integrity criterion " $E$ " and insulation criterion "I" after 105 minutes. The reason for the occurrence of the crack was explained in the report [49] by welding of the reinforcement at this location. In Section 7.3 of this work it will be shown that the available reinforcement amount was not sufficient. The reinforcement would have ruptured even if it had been continuous at this location. This test shows again that the consideration of the load bearing criterion " $\mathrm{R}$ " is not sufficient. With the design methods currently available gaping cracks that occurred at the first Munich test and the FRACOF test cannot be prevented and cause at least integrity and insulation failure. The method presented in this work deals with these problems. 


\section{Thermal loading}

In this chapter the determination of the temperature distribution in the slab and beams is explained. The effects on the structure are discussed and suggestions are made as to how they can be simplified. The temperature distribution depends on the temperature development of the surrounding gas. In many countries, the standard fire curve is generally used to describe this temperature development. The curve was internationally agreed in ISO 834 and was taken over as a standard in the Eurocodes. It was established to standardise fire tests and to be able to categorise products with fire safety requirements. The specifications in building regulations are also usually based on the ISO fire. For example, if a fire wall has to fulfil the standard R90, this means that it must not collapse for ninety minutes exposed to the ISO fire. In the annexes of Eurocode 1 [24] some information is given about how natural fires can be considered. The use of natural fires, however, would mean that every part in the building has to be proved with the considered fire curve. Every fire door, fire shutter, cable exit etc. would have to be tested in extra fire tests. According to the German national annex of the Eurocode [25] the ISO fire has to be used in general. The usage of the ISO fire also simplifies the numerical simulations considerably since at least one-third of the simulation work drops out. If different natural fire scenarios and mechanical load cases have to be considered, the effort is even exponentially higher since every load case has to be combined with every fire scenario. For these reasons, the standard fire is used in this work. With a little more effort, the presented method can, however, also be used for natural fire curves. Only the heating phase can be considered, however, since permanent plastic deformations cannot be considered with the method.

\subsection{Thermal analysis}

In the Eurocodes several regulations can be found to determine the temperature distribution in crosssections. Regulations are available for general numerical simulations. Simple calculation methods can be found only for steel cross-sections and composite slabs. The method in DIN EN 1994-1-2, Annex D [32] for calculating temperatures in composite slabs is discussed in Section 4.1.2. For numerical simulations the following regulations can be found in the Eurocodes and are used in this work:

- The thermal properties of steel and concrete are used according to DIN EN 1994-1-2, 3.3.

- For concrete the thermal properties for normal weight concrete with siliceous aggregates are used.

- For the specific heat a moisture of $3 \%$ is assumed according to DIN EN 1994-1-2, 3.3.2 (8).

- For the thermal conductivity the upper limit is used as recommended in DIN EN 1994-1-2, 3.3.2 (9) and adopted in the German national annex [33].

- The coefficient of heat transfer by convection is generally $\alpha_{c}=25 \mathrm{~W} / \mathrm{m}^{2} \mathrm{~K}$ for surfaces exposed to the standard fire curve (DIN EN 1991-1-2, 3.2 .1 (2)). This assumption has to be adjusted for parts of the troughs as explained in Section 4.1.2.

- For the unexposed side the coefficient of heat transfer by convection is set to $\alpha_{c}=9 \mathrm{~W} / \mathrm{m}^{2} \mathrm{~K}$ including effects of heat transfer by radiation (DIN EN 1991-1-2, 3.1 (5)). 
- The emissivity coefficient for steel and concrete related to the surface of the member is generally set to $\varepsilon_{m}=0.7$ according to DIN EN 1994-1-2, 2.2 (2).

- Shadow effects are allowed to be taken into account (DIN EN 1991-1-2, 3.1 (7)). These are considered in this work for parts of the troughs as explained in Section 4.1.2.

These assumptions are used for the preparation of the tables and approximation formulas for the substitute temperature loading in Section 4.2. They have to be adjusted slightly for modelling the fire tests in order to fit the simulations to the test data. For the thermal analysis in this work the finite element software Ansys Workbench version 12.1.0 is used.

\subsubsection{Full concrete slabs}

The thermal analysis of full concrete slabs is relatively simple since the heat transfer is a one-dimensional problem. Ansys only provides two and three-dimensional analyses, therefore, a two dimensional system is chosen to determine the temperature distribution in the cross-section. In order to validate the simulation, the results are compared with available data from the literature. In DIN EN 1994-1-2, D.5 [32] a table is given with temperatures of a $100 \mathrm{~mm}$ thick concrete slab for different time steps exposed to the standard fire. These values are compared with a numerical simulation with the assumptions described above. The results are shown in Figure 4.1. It can be seen that the numerical simulations are in good agreement with the values from the Eurocode $(E C)$.

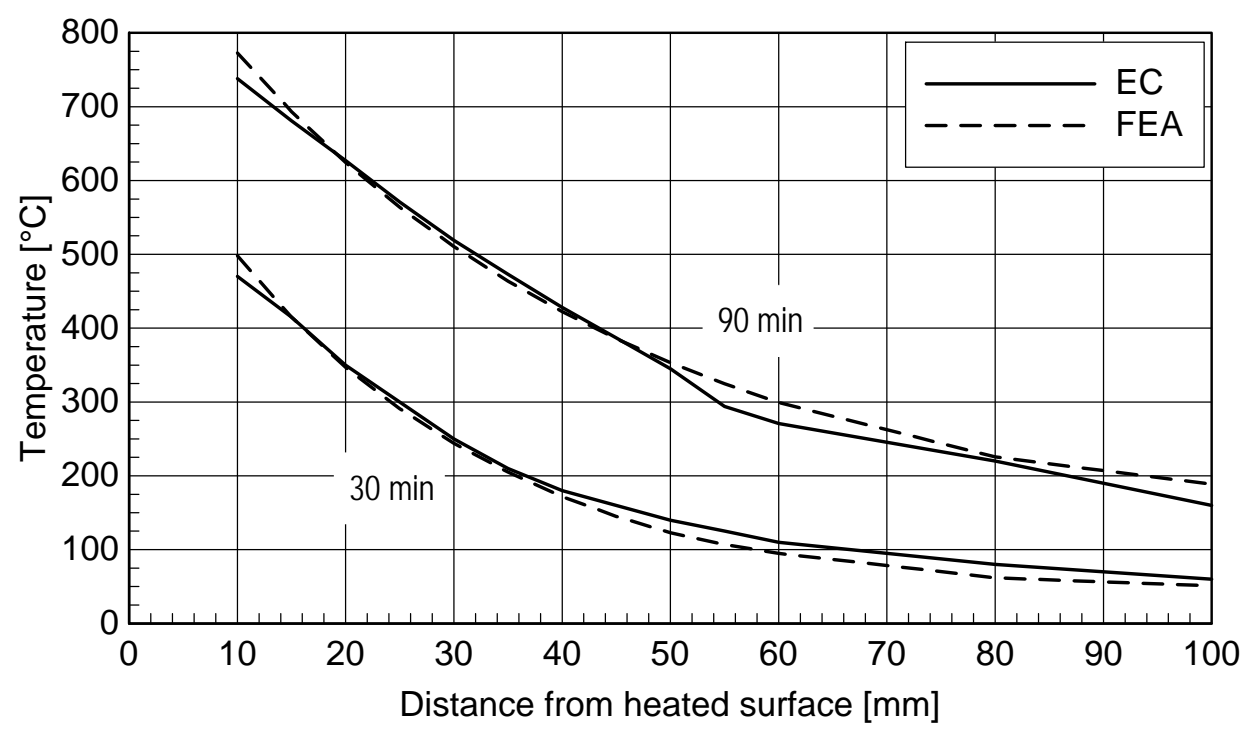

Fig. 4.1: Comparison of the temperature distributions from Eurocode data with FEA

Figure 4.2 shows a comparison between test data from the first Munich fire test with numerical simulations. In the experiment a precast slab with in-situ concrete topping with an overall depth of $12 \mathrm{~cm}$ was tested. The gas temperature development in the test almost followed the standard fire curve. More information about the test can be found in [42] and [50]. In the simulations the thermal properties and heat transfer coefficients from the Eurocodes are used and the standard fire curve is used for the heat exposure. It can be seen that the assumptions in the Eurocode lead to temperature distributions that fit well to the test data. It can be confirmed that the parameters given in the Eurocode are suitable for simulating temperature distributions in full concrete slabs exposed to the standard fire. No adjustments need to be 
made in order to use the parameters for further studies.

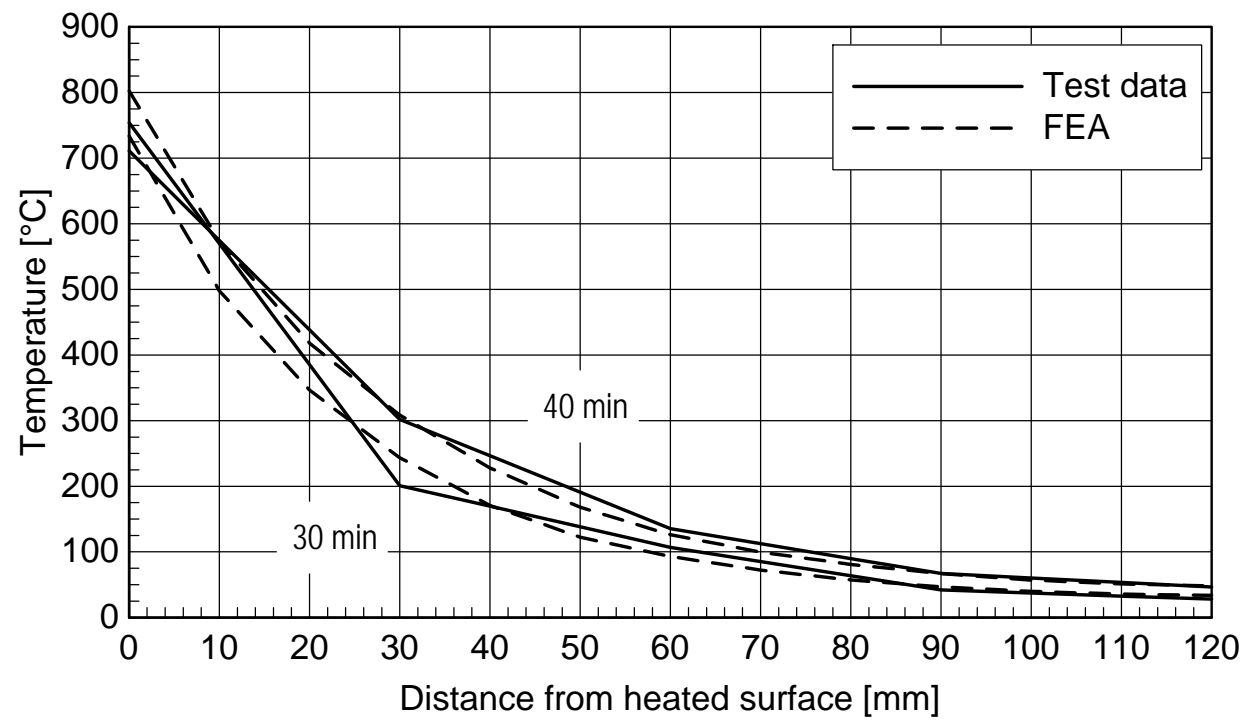

Fig. 4.2: Comparison of temperatures from first Munich test with FEA

\subsubsection{Composite slabs}

The behaviour of composite slabs is investigated on two examples. For a re-entrant trough profiled steel sheeting the Holorib HR51 profile is considered and for an open trough profiled steel sheeting the Cofraplus 60 profile. The cross-sections can be seen in Figure 4.3. Both slab systems are very common in Germany and other European countries and were therefore chosen for this work. A comparison is firstly made between different assumptions in the Eurocodes for the calculation of temperatures in composite slabs. Secondly, fire tests are modelled in order to be able to simulate temperature distributions for a further mechanical analysis. Out of these comparisons a conclusion is drawn about which parameters shall be chosen in the thermal analysis in order to derive the substitute temperature loading for the general case.
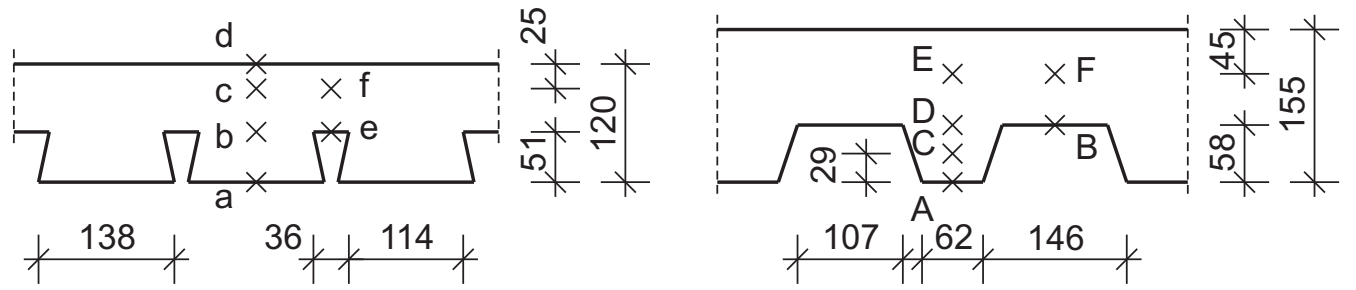

Fig. 4.3: Composite slab cross-sections with re-entrant (left) and open (right) trough profile steel sheeting

In DIN EN 1994-1-2, Annex D.2 [32] a simple method is given to calculate temperatures in composite slabs that are subjected to the standard fire. The temperature prediction is included in a method to calculate the hogging moment resistance of composite slabs. Temperatures can be determined for the lower flange, the upper flange and the web of the steel decking as well as for reinforcement bars in the ribs. In this method, the temperatures of the steel decking only depend on geometry and whether normal or light weight concrete is used. The temperature of the reinforcement bar also depends on the location 
in the rib. There is no dependency on the slab thickness, the moisture and the thermal conductivity of the concrete like in numerical simulations.

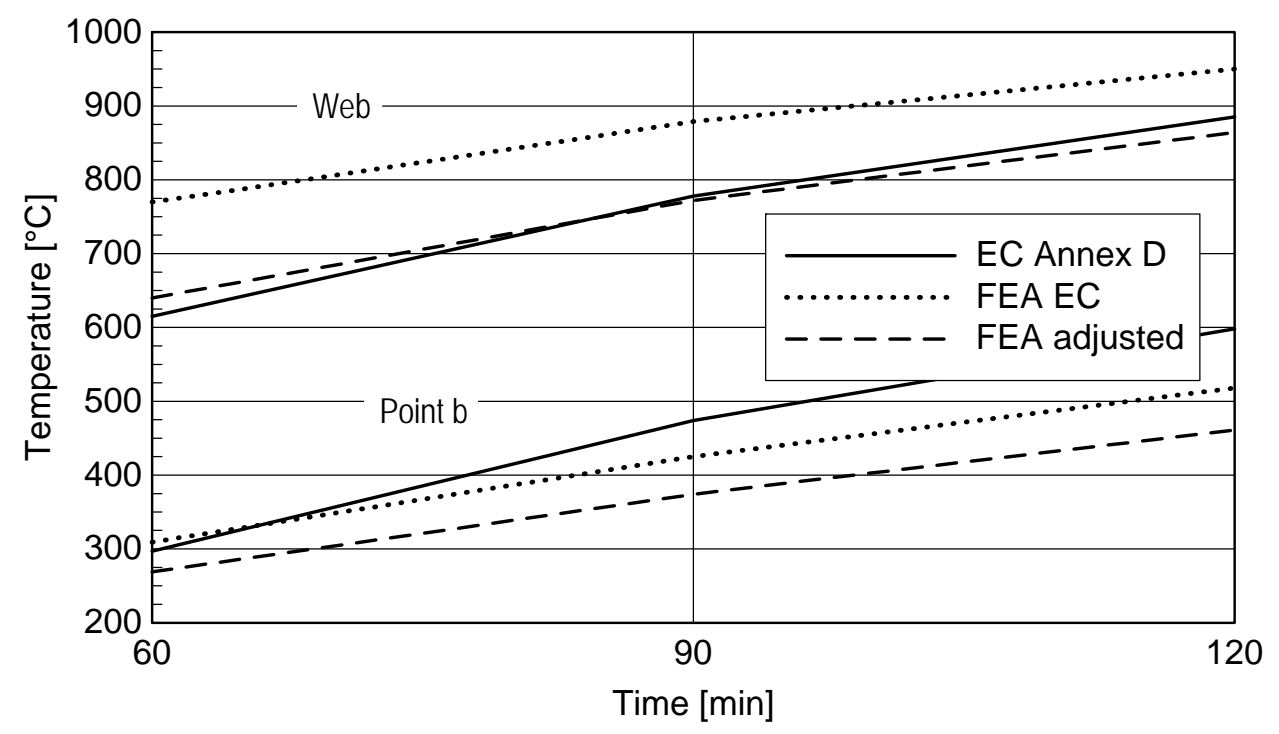

Fig. 4.4: Temperatures according to Eurocode compared with numerical simulations for Holorib slab

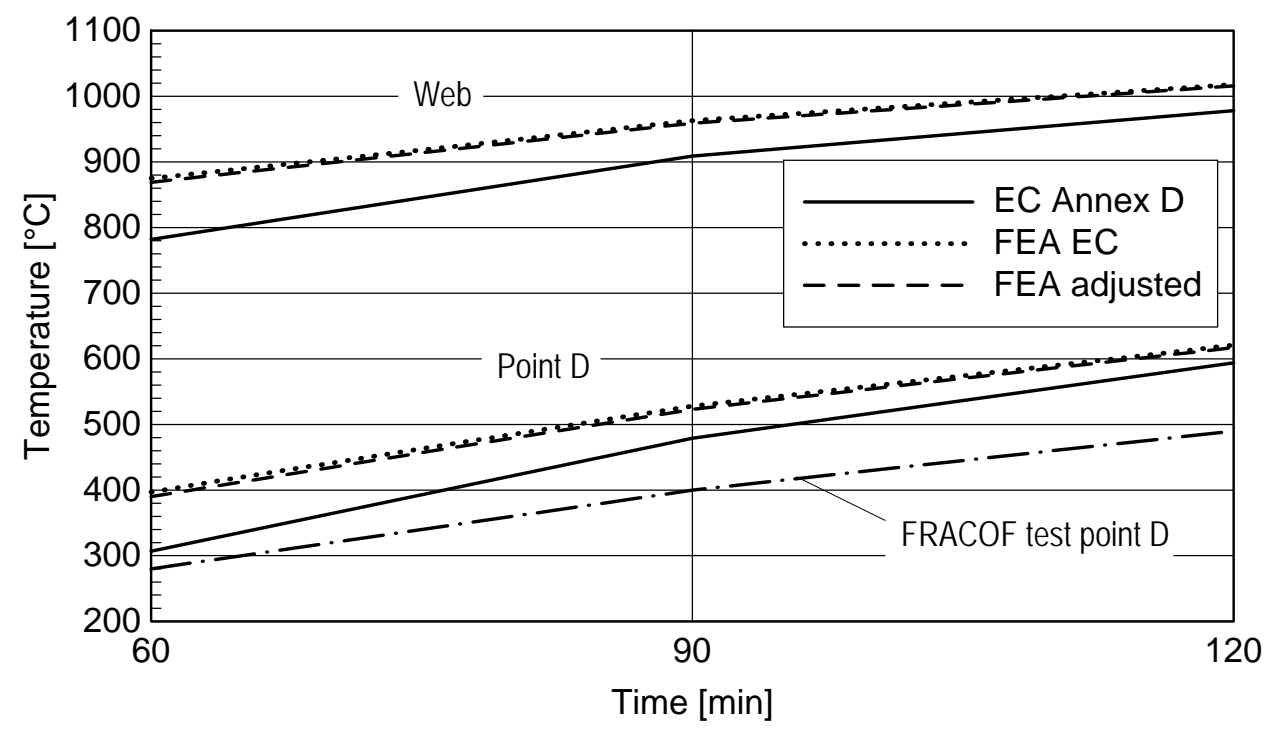

Fig. 4.5: Temperatures according to Eurocode compared with numerical simulations for Cofraplus slab

In Figure 4.4 and 4.5 the solid lines show the temperatures of a Holorib slab and a Cofraplus slab respectively, that can be found with this method. As an example, two points are considered: the web of the steel sheeting and a reinforcement bar in the middle of a rib at the same hight as the upper flange. This location is marked with "Point b" for the Holorib slab and "Point D" for the Cofraplus slab as shown in Figure 4.3. The dotted lines in Figures 4.4 and 4.5 show a numerical simulation following the regulations of the Eurocodes. Shadow effects for the upper flange and the web are taken into account by multiplying the emissivity coefficient $\varepsilon_{m}$ with a view factor. This factor $\Phi$ can be calculated by the Rule of Hottel that is described in [37]. The proportionate amount of radiation that reaches Surface $j$ coming 
through Surface $i$ can be calculated by following equation:

$$
F_{i j}=\frac{\overline{A D}+\overline{B C}-\overline{A C}-\overline{B D}}{2 \overline{A B}}
$$

The meanings of the variables can be seen in Figure 4.6. Equation (4.1) coincides with the configuration or view factor that is given in DIN EN 1994-1-2, Annex D, Equation (D.3). The factor of the Eurocode is only valid for the upper flange of composite slabs whereas the Rule of Hottel describes the general case.

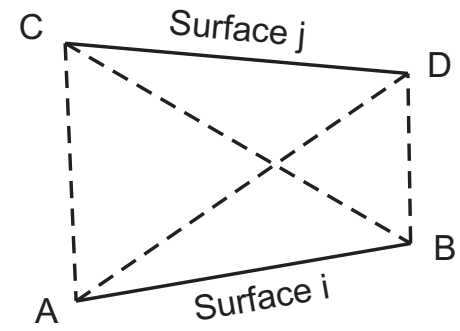

Fig. 4.6: Rule of Hottel according to [37]

For the considered slab geometries the following view factors can be found:

Table 4.1: View factors for simulations according to the Eurocodes

\begin{tabular}{|l|c|c|}
\hline & Upper flange & Web \\
\hline Holorib & 0.110 & 0.077 \\
\hline Cofraplus & 0.728 & 0.556 \\
\hline
\end{tabular}

In Figures 4.4 and 4.5 it can be seen that simulations with the regulations of the Eurocodes deliver higher temperatures in most cases than the method of Annex D. The Eurocode is not consistent in this case and needs to be corrected. The reason for the discrepancy probably lies in the fact that the method in Annex D has been calibrated with experiments. There occur considerably lower temperatures than at simulations according to the Eurocodes. This can be seen for example in Figure 4.5 on the dash-dot line. The line shows the test data of Point $\mathrm{D}$ of the FRACOF test. Although the gas temperature followed the standard fire curve, the temperatures in the slab are lower than predicted by the Eurocode. One of the reasons is that not only the radiative part of the heat transfer reduces in the troughs but also the convection can be significantly lower. The hot gas inside the troughs cannot move and be exchanged as quickly as at directly exposed surfaces. Franssen [37] therefore suggests, also to reduce the coefficient of heat transfer by convection $\alpha_{c}$ by multiplying it with the view factors. The results of simulations where this is included can be seen in Figures 4.4 and 4.5 on the dashed lines. In the Holorib slab the temperatures are reduced considerably due to the re-entrant shape of the trough. In the Cofraplus slab the effect is small.

The Holorib profile has been used in the second Munich fire test. Like in the first test the overalldepth of the slab was $12 \mathrm{~cm}$. The gas temperature development approximately followed the standard fire curve but remained slightly below. Details about the test can be found in [42] and [50]. For the report of the project [42] the test has already been modelled in Abaqus. In this model a temperature dependent emissivity factor has been used, in order to take into account that the steel sheeting in the test has been galvanised. At low temperatures the emissivity is very low with $\varepsilon_{m}=0.11$ since the zinc surface strongly reflects the radiation. At $420^{\circ} \mathrm{C}$ the zinc melts and the emissivity of the surface suddenly rises 
to $\varepsilon_{m}=0.7$. View factors have also been used inside the troughs for the emissivity as described above. The convection coefficient inside the troughs has been changed to $\alpha_{c}=10 \mathrm{~W} / \mathrm{m}^{2} \mathrm{~K}$ and outside to $\alpha_{c}=$ $35 \mathrm{~W} / \mathrm{m}^{2} \mathrm{~K}$. With these assumptions the temperature development could be satisfactorily reproduced.

A temperature dependent emissivity cannot be input in Ansys. The temperature development can therefore not be reproduced over the whole test period as well as with Abaqus. For the requirements of the method in this work, however, it is not essential that the development over the time is correct. The right temperature distribution inside the cross-section at a certain time is important, since only selected times are considered. The time that is chosen to validate the method on the Munich tests is forty minutes after lighting. At this time in both tests the gas temperature in the furnace reached its highest level. For the gas temperature in the simulation, the average temperature measured in the test is used. In order to align the simulations to the test data, the heat transfer coefficients are adjusted. At the unexposed side of the slab a change of the convection coefficient to $\alpha_{c}=45 \mathrm{~W} / \mathrm{m}^{2} K$ was necessary. The view factors used for the simulation of the second Munich test and the FRACOF test, which are discussed later on, are shown in Table 4.2. The factors are used both for the emissivity and the convection.

Table 4.2: View factors for simulation of fire tests

\begin{tabular}{|l|c|c|c|}
\hline & Upper flange & Web & Lower flange \\
\hline Holorib: second Munich test & 0.25 & 0.20 & 0.80 \\
\hline Cofraplus: FRACOF test & 0.45 & 0.14 & 0.22 \\
\hline
\end{tabular}

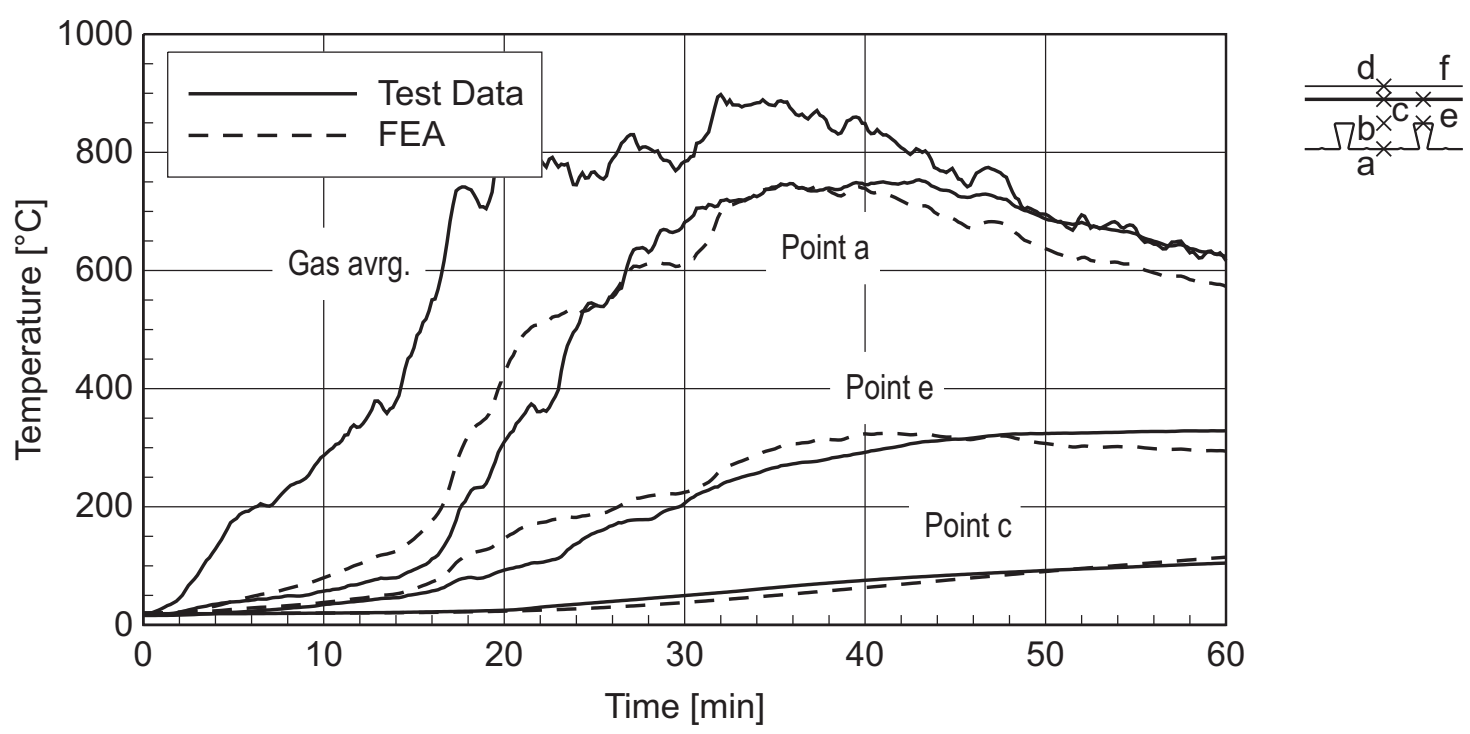

Fig. 4.7: Comparison of temperatures from second Munich test with FEA

A comparison of single simulation results with data of the second Munich test can be seen in Figure 4.7. It is noticeable that the heat transfer coefficients had to be changed significantly. Possible reasons for the big discrepancies are, on the one hand, the influence of the zinc surface as described above and, on the other hand, debonding of the steel sheeting. The steam that develops in the concrete is stopped from escaping by the sheeting. A gas pressure builds up which disconnects the sheeting from the concrete. The void between the two materials acts like an insulation layer and slows down the heat transfer. A further reason for the different results can be the moisture of the concrete. For the simulations a general moisture of $3 \%$ is assumed. The actual moistures of the test-specimens are not known. In 
the simulations the moisture is taken into account by a peak in the curve of the specific heat at $100^{\circ} \mathrm{C}$. This slows down the heating of the whole cross-section but does not reproduce a temperature plateau of $100^{\circ} \mathrm{C}$ at a certain location as seen in tests when the water in the concrete is boiling. All these effects are difficult to include in a thermal analysis directly. Therefore they are taken into account by adjusting the view factors.

A similar behaviour could be seen at the FRACOF test, where a Cofraplus 60 slab was used. The cross-section of the tested slab and the location of the measuring points are shown in Figure 4.3. The temperature-time curve of the gas in the furnace followed the standard fire curve. The time of the test that is considered in this work is 105 minutes, since the large crack at the centre of the slab occurred at this time. The standard fire curve is used in the simulation. The convection coefficient on the top surface of the slab is set to $\alpha_{c}=50 \mathrm{~W} / \mathrm{m}^{2} \mathrm{~K}$. The view factors which are used to multiply the radiation and convection coefficients of the exposed surface are given in Table 4.2. A comparison of the simulation results and the test data is shown in Table 4.3. Points A and B in the steel sheeting have not been measured in the test since the thermocouples failed very early at these locations. As in the simulation of the second Munich fire test it can be seen, that the simulation results can only be aligned to the test data by large adjustments of the heat transfer coefficients. The reasons are again probably due to the zinc surface, debonding of the steel sheeting and an unknown moisture rate of the concrete.

Table 4.3: Comparison of temperatures after 105 minutes from FRACOF test with FEA

\begin{tabular}{|c|c|c|}
\hline Point & Test data & FEA \\
\hline & {$\left[{ }^{\circ} \mathrm{C}\right]$} & {$\left[{ }^{\circ} \mathrm{C}\right]$} \\
\hline A & - & 925 \\
\hline B & - & 927 \\
\hline C & 650 & 653 \\
\hline D & 420 & 460 \\
\hline E & 195 & 207 \\
\hline F & 290 & 276 \\
\hline Top side & 90 & $87-102$ \\
\hline
\end{tabular}

In summary, it can be said that the simple calculation model of Eurocode Annex D, numerical simulations according to the Eurocodes and fire tests deliver significantly different results for composite slabs. Numerical simulations with parameters given in the Eurocode lead to the highest temperatures and fire tests to the lowest. The Eurocode needs to be changed so that the simple method would deliver similar or slightly higher temperatures than the numerical simulations. The main reasons for the different temperatures in the simulations are the unconsidered effects of the zinc surface, debonding of the steel sheeting and reduced convection in the troughs. The first two effects should not generally be taken into account. It cannot be assured that these occur in any case. Not every steel sheeting is necessarily galvanised and holes in the sheeting can release steam and prevent debonding. However, the convection in the troughs is always lower than at directly exposed surfaces. The proposal of Franssen [37] therefore is used in this work; the coefficients of convection shall be reduced by the same view factors as the coefficients of radiation at shadowed surfaces. 


\subsection{Substitute thermal loading on slabs}

This is the central section in this work. Here it is described how the thermal analyses can be avoided and replaced by a substitute thermal loading. Tables are worked out for Holorib and Cofraplus 60 slabs subjected to the standard fire and simple approximation formulas are given to calculate the substitute thermal loading. The methods described in this section can be easily applied to any other cross-section and fire scenario.

\subsubsection{Derivation}

A distribution of high temperatures $\theta_{\text {real }}$ inside a cross-section has two effects as shown in Figure 4.8: It causes thermal elongation which can be expressed by thermal strains $\varepsilon(\theta)$, and it reduces the stiffness expressed by the Young's modulus $E(\theta)$. If the elongation is restrained, stresses will occur in the crosssection, which will be called non-linear thermal stresses $\sigma_{\text {nonl }}(\theta)$. These stresses can be calculated by multiplying $E(\theta)$ with $\varepsilon(\theta)$. The stress distribution can be split into a linear part $\sigma_{\theta, \text { lin }}$ and a part of selfequilibrating stresses $\sigma_{\theta, \text { self }}$. The fictive linear stress distribution $\sigma_{\theta, \text { lin }}$ causes the same deformations in a beam as the real stress distribution $\sigma_{\text {nonl }}(\theta) . \sigma_{\theta \text {,self }}$ does not cause any deformation. It only can soften a cross-section if, for example, in a concrete cross-section $\sigma_{\theta, \text { self }}$ reaches the tensile strength of the concrete. $\sigma_{\theta, \text { lin }}$ can again be split. At first, an arbitrary Young's modulus $E_{\text {subs }}$ is divided out. Then the remaining strains are split into a constant part $\varepsilon_{\theta, \text { subs }}$ and a linear part $\kappa_{\theta, s u b s}$ with zero-crossing in the neutral axis of the cross-section. The thermal strain $\varepsilon_{\theta, \text { subs }}$ only causes elongation in a beam, no bending whilst $\kappa_{\theta, \text { subs }}$ only causes bending, no elongation or shortening of the neutral axis, and therefore will be called thermal curvature.

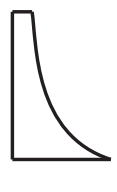

$\theta_{\text {rea }}$

$\sigma_{\theta, \text { lin }}$

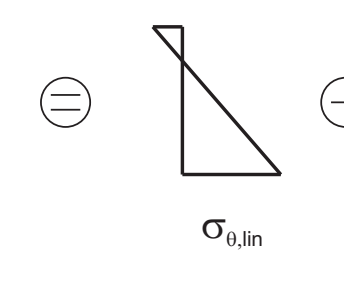

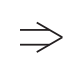
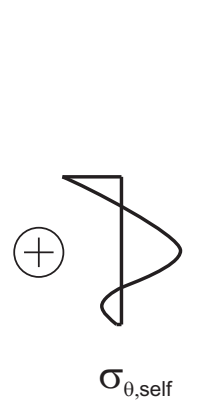

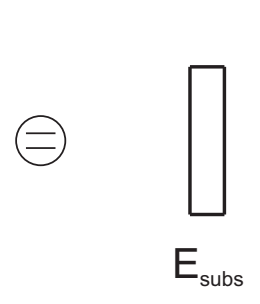

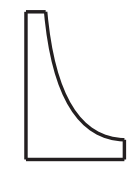

$\varepsilon(\theta)$

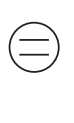

$\mathrm{E}(\theta)$

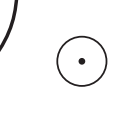

)

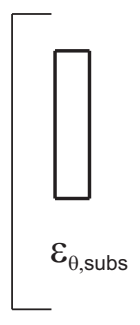

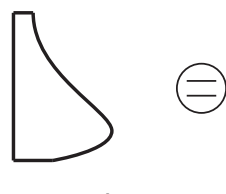

$\sigma_{\text {nonl }}(\theta)$

Fig. 4.8: Derivation of the substitute thermal loading

If $\varepsilon_{\theta, s u b s}$ and $\kappa_{\theta, \text { subs }}$ are applied to any beam, they will cause the same deformations as a real temperature distribution $\theta_{\text {real }}$ would do to the originally considered beam. Height and stiffness of the beam can be arbitrary, only the length must be the same. The reason is, that the overall elongation $u$ caused by $\varepsilon_{\theta, \text { subs }}$ and the deflection in the middle of the beam $w_{m}$ caused by $\kappa_{\theta, \text { subs }}$ only depend on the length $l$ of a beam as shown in Equations (4.2) and (4.3). This means, that the stiffness of a structure can be determined separately from the deformations caused by temperature changes.

$$
u=\varepsilon_{\theta, s u b s} \cdot l
$$




$$
w_{m}=\frac{\kappa_{\theta, s u b s} \cdot l^{2}}{8}
$$

For finite element programs where the thermal strain and curvature cannot be input directly, these can easily be transformed into temperature loadings. With an arbitrary chosen coefficient of thermal expansion $\alpha_{T}$, the uniform temperature increase $\Delta \theta_{\text {unif }}$ can be calculated by Equation (4.4), and the temperature gradient between the bottom and top surface of the slab $\Delta \theta_{\text {grad }}$ with the depth $h$ is obtained by Equation (4.5).

$$
\begin{aligned}
\Delta \theta_{\text {unif }} & =\frac{\varepsilon_{\theta, \text { subs }}}{\alpha_{T}} \\
\Delta \theta_{\text {grad }} & =\frac{\kappa_{\theta, \text { subs }}}{\alpha_{T}} \cdot h
\end{aligned}
$$

The temperature distribution $\theta_{\text {real }}$ can be obtained by numerical simulations as described in Section 4.1. Formulas for the thermal elongation $\varepsilon(\theta)$ of concrete as a function of the temperature are given in DIN EN 1994-1-2, 3.3.2 [32] and used in this work. The temperature dependent Young's modulus of concrete is slightly more difficult. In the Eurocodes, Young's moduli for concrete are only available for ambient temperature. For elevated temperatures only stress-strain curves are given. These show no linear character from the zero strain. In order to obtain the same results in numerical simulations with the full stress-strain curves and the method in this work, the same stiffness needs to be used. The Young's modulus therefore needs to be derived from the non-linear stress-strain curves. For ambient temperature an approach for that can be found in DIN EN 1992-1-1, 3.1.5 [26]. There the secant modulus is used, where the secant intersects the stress-strain curve at $40 \%$ of the compressive strength. This approach is adopted in this work as shown in Figure 4.9.

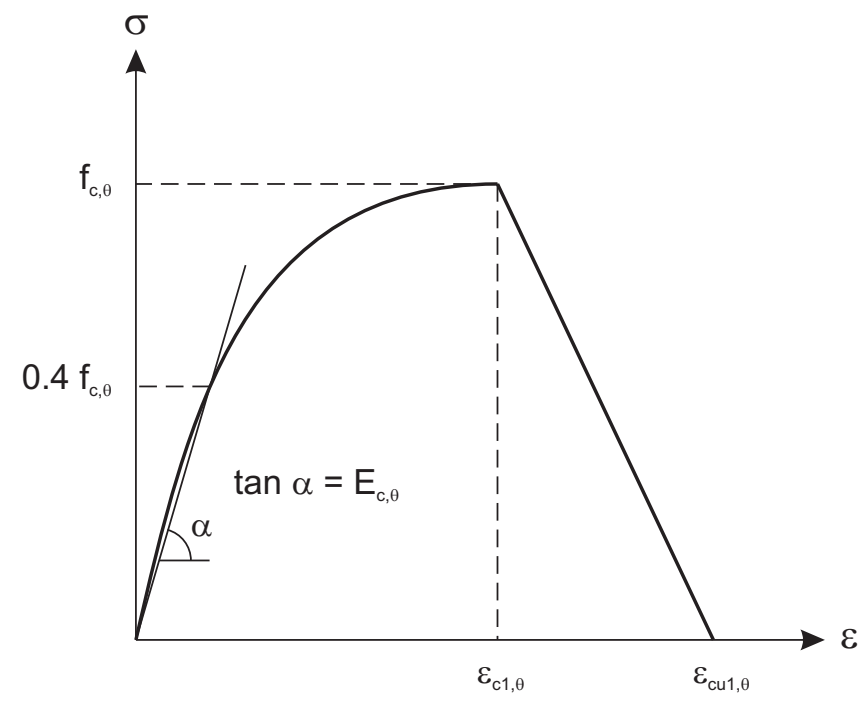

Fig. 4.9: Young's modulus of concrete under elevated temperatures (adopted from [26])

The compression part of the stress-strain curve of concrete under elevated temperatures is given in DIN EN 1992-1-2, 3.2.2.1 [27] with following equation:

$$
\sigma=\frac{3 \varepsilon f_{c, \theta}}{\varepsilon_{c 1, \theta}\left[2+\left(\frac{\varepsilon}{\varepsilon_{c 1, \theta}}\right)^{3}\right]}
$$


The stress is set to $40 \%$ of the concrete strength. An equation can then be found to derive the corresponding strain $\varepsilon_{0.4 f c \theta}$ of the intersection point of the Young's modulus.

$$
\begin{aligned}
& \sigma=0.4 f_{c, \theta} \\
& \frac{3 \varepsilon_{0.4 f c \theta} \cdot f_{c, \theta}}{\varepsilon_{c 1, \theta}\left[2+\left(\frac{\varepsilon_{0.4 f c \theta}}{\varepsilon_{c 1, \theta}}\right)^{3}\right]}=0.4 f_{c, \theta} \\
& \frac{3 \varepsilon_{0.4 f c \theta}}{\varepsilon_{c 1, \theta}\left[2+\left(\frac{\varepsilon_{0.4 f c \theta}}{\varepsilon_{c 1, \theta}}\right)^{3}\right]}-0.4=0
\end{aligned}
$$

In Equation (4.9) it can be seen that the strain of the intersection point is independent of the concrete strength. It only depends on the temperature, expressed by the value $\varepsilon_{c 1, \theta}$. This value is tabulated as a function of temperature in DIN EN 1992-1-2, Table 3.1 [27].

With:

$$
\begin{aligned}
E_{c, \theta} & =\frac{0.4 f_{c, \theta}}{\varepsilon_{0.4 f c \theta}} \\
k_{E, \theta} E_{c, 20} & =\frac{0.4 k_{c, \theta} \cdot f_{c, 20}}{\varepsilon_{0.4 f c \theta}}
\end{aligned}
$$

and:

$$
E_{c, 20}=\frac{0.4 f_{c, 20}}{\varepsilon_{0.4 f c 20}}
$$

follows:

$$
k_{E, \theta}=\frac{k_{c, \theta} \cdot \varepsilon_{0.4 f c 20}}{\varepsilon_{0.4 f c \theta}}
$$

With Equation (4.13) a reduction factor for the Young's modulus of concrete under elevated temperatures is found. It only depends on the temperature, not on the strength of the concrete. In Table 4.4 the derived Young's moduli under $20^{\circ} \mathrm{C}$ for some concrete strength classes are prepared, and in Table 4.5 the reduction factors for different temperatures. The values in both tables are only valid for normal weight concrete with siliceous aggregates.

Table 4.4: Young's moduli of concrete under $20^{\circ} \mathrm{C}$ according to the non-linear stress-strain curves of Eurocode 2 [27]

\begin{tabular}{|cc|c|c|c|c|c|}
\hline$f_{c, 20}$ & {$\left[\mathrm{~N} / \mathrm{mm}^{2}\right]$} & 20 & 25 & 30 & 35 & 40 \\
\hline$E_{c, 20}$ & {$\left[\mathrm{~N} / \mathrm{mm}^{2}\right]$} & 11884 & 14855 & 17826 & 20797 & 23768 \\
\hline
\end{tabular}

Table 4.5: Reduction factors for the Young's modulus of concrete under elevated temperatures

\begin{tabular}{|ll|c|c|c|c|c|c|}
\hline$\theta_{c}$ & {$\left[{ }^{\circ} \mathrm{C}\right]$} & 20 & 100 & 200 & 300 & 400 & 500 \\
\hline$k_{E, \theta}=E_{c, \theta} / E_{c, 20}$ & {$[-]$} & 1.000 & 0.625 & 0.432 & 0.304 & 0.188 & 0.100 \\
\hline$\theta_{c}$ & {$\left[{ }^{\circ} \mathrm{C}\right]$} & 600 & 700 & 800 & 900 & 1000 & 1100 \\
\hline$k_{E, \boldsymbol{\theta}}=E_{c, \theta} / E_{c, 20}$ & {$[-]$} & 0.045 & 0.030 & 0.015 & 0.008 & 0.004 & 0.001 \\
\hline
\end{tabular}




\subsubsection{Full concrete slabs}

For full concrete slabs the determination of the substitute thermal strains $\varepsilon_{\theta, s u b s}$ and curvatures $\kappa_{\theta, \text { subs }}$ of Figure 4.8 is relatively simple. It is solely a one-dimensional problem. Temperatures along a linear section through the depth of a full concrete slab are similar in each direction and are independent of the location of the section. Integrations can easily be done along this line, for example with the trapezoidal rule. The other two dimensions of a slab are simply included by considering a rectangular cross-section with unit width.

The thermal strains and curvature can be integrated over this cross-section and a thermal normal force $N_{\theta}$ and a thermal bending moment $M_{\theta}$ are obtained with Equations (4.15) and (4.17). Where $h$ is the depth of the slab and $z_{n}$ is the distance from the neutral axis of the hot cross-section.

$$
\begin{aligned}
& N=\int_{(A)} \sigma d A \\
& N_{\theta}=\int_{(A)} \sigma_{\text {nonl }}(\theta, z) d A=\int_{-h / 2}^{h / 2} E(\theta, z) \cdot \varepsilon(\theta, z) d z \\
& M=\int_{(A)} \sigma \cdot z_{n} d A \\
& M_{\theta}=\int_{(A)} \sigma_{\text {nonl }}(\theta, z) \cdot z_{n} d A=\int_{-h / 2}^{h / 2} E(\theta, z) \cdot \varepsilon(\theta, z) \cdot z_{n} d z
\end{aligned}
$$

These forces would occur in a beam whose deformations are restrained against both elongation and bowing. Both forces act on the neutral axis of the cross-section, as it is commonly defined in engineering mechanics. The neutral axis of a rectangular cross-section with uniform stiffness lies in the centre of the cross-section. If the Young's modulus changes non-uniformly, like is in heated slabs, the stiffness becomes non-uniform and the neutral axis moves. The distance of the neutral axis from an arbitrary location can be calculated by Equation (4.20). This location is chosen usefully at the bottom of the slab.

$$
\begin{aligned}
E A & =\int_{(A)} E d A \\
E S_{y} & =\int_{(A)} E \cdot z d A \\
a_{z n} & =\frac{E S_{y}}{E A}=\frac{\int_{-h / 2}^{h / 2} E(\theta, z) \cdot z d z}{\int_{-h / 2}^{h / 2} E(\theta, z) d z}
\end{aligned}
$$

With the thermal normal force and bending moment, the substitute thermal strain and curvature can be determined. For the determination of the required bending stiffness $E I_{y}$, it is important that $z_{n}$ again is the distance from the neutral axis of the hot cross-section.

$$
\varepsilon_{\theta, s u b s}=\frac{N_{\theta}}{E A}
$$




$$
\kappa_{\theta, \text { subs }}=\frac{M_{\theta}}{E I_{y}}
$$

With:

$$
E I_{y}=\int_{(A)} E \cdot z_{n}^{2} d A=\int_{-h / 2}^{h / 2} E(\theta, z) \cdot z_{n}^{2} d z
$$

The substitute thermal strain and curvature only depend on the depth and the temperature distribution in the cross-section. They are, first of all, independent of concrete strength classes. The temperature distribution only depends on the depth of the slab and the fire scenario. If for the fire scenario the standard fire is used, it will mean that the only remaining parameter is the slab depth. This can be proved as follows:

$$
\begin{aligned}
& \varepsilon_{\theta, s u b s}= \frac{N_{\theta}}{E A}=\frac{\int_{-h / 2}^{h / 2} E(\theta, z) \cdot \varepsilon(\theta, z) d z}{\int_{-h / 2}^{h / 2} E(\theta, z) d z}=\frac{\int_{-h / 2}^{h / 2} k_{E, \theta}(z) \cdot E_{c, 20} \cdot \varepsilon(\theta, z) d z}{\int_{-h / 2}^{h / 2} k_{E, \theta}(z) \cdot E_{c, 20} d z} \\
&= \frac{\int_{-h / 2}^{h / 2} k_{E, \theta}(z) \cdot \varepsilon(\theta, z) d z}{h / 2} k_{E, \theta}(z) d z \\
&-h / 2
\end{aligned}
$$

The only remaining variables are $h, k_{E, \theta}$ and $\varepsilon_{\theta}$. They are all independent of the concrete strength class as described above. The same is true for the curvature:

$$
\kappa_{\theta, s u b s}=\frac{M_{\theta}}{E I_{y}}=\frac{\int_{-h / 2}^{h / 2} k_{E, \theta}(z) \cdot \varepsilon(\theta, z) \cdot z_{n} d z}{\int_{-h / 2}^{h / 2} k_{E, \theta}(z) \cdot z_{n}^{2} d z}
$$

This correlation allows very simple tables and diagrams for $\varepsilon_{\theta \text {,subs }}$ and $\kappa_{\theta \text {,subs }}$ to be determined. Such a table is worked out for common slab depths and fire resistant classes as shown in Table 4.6 and, as an example, a diagram for $\kappa_{\theta, \text { subs }}$ is shown in Figure 4.10. In Appendix A one detailed example for $h=12 \mathrm{~cm}$ and $\mathrm{R} 30$ is shown.

Table 4.6: Substitute thermal loading on full concrete slabs

\begin{tabular}{|c|c|c|c|c|c|c|}
\hline Slab depth & \multicolumn{2}{|c|}{$\mathrm{R} 30$} & \multicolumn{2}{c|}{$\mathrm{R} 60$} & \multicolumn{2}{c|}{$\mathrm{R} 90$} \\
\hline $\mathrm{h}$ & $\varepsilon_{\theta, \text { subs }}$ & $\kappa_{\theta, \text { subs }}$ & $\varepsilon_{\theta, \text { subs }}$ & $\kappa_{\theta, \text { subs }}$ & $\varepsilon_{\theta, \text { subs }}$ & $\kappa_{\theta, \text { subs }}$ \\
\hline$[\mathrm{cm}]$ & {$[\%$ o $]$} & {$[1 / \mathrm{km}]$} & {$[\%$ o $]$} & {$[1 / \mathrm{km}]$} & {$[\%$ ] } & {$[1 / \mathrm{km}]$} \\
\hline 10 & 1.062 & 45.25 & 2.069 & 71.31 & 3.169 & 87.41 \\
\hline 12 & 0.791 & 32.65 & 1.576 & 50.58 & 2.255 & 65.19 \\
\hline 14 & 0.624 & 24.47 & 1.213 & 38.09 & 1.796 & 49.22 \\
\hline 16 & 0.514 & 18.84 & 0.966 & 29.90 & 1.446 & 38.53 \\
\hline 18 & 0.437 & 14.85 & 0.796 & 24.02 & 1.188 & 31.47 \\
\hline 20 & 0.379 & 11.92 & 0.678 & 19.80 & 1.004 & 26.49 \\
\hline
\end{tabular}




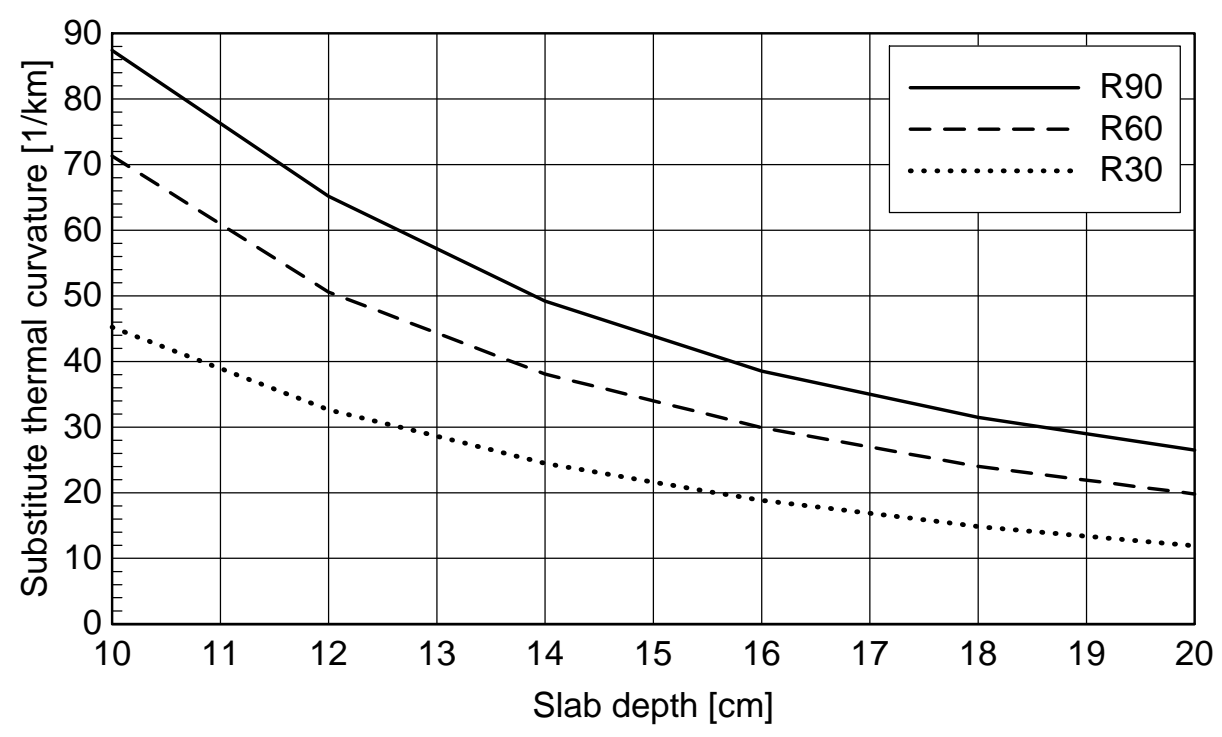

Fig. 4.10: Substitute thermal curvature $\kappa_{\theta, \text { subs }}$ on full concrete slabs

The values of $\varepsilon_{\theta, \text { subs }}$ and $\kappa_{\theta, \text { subs }}$ can also be expressed by following equations with the slab depth $h$ in $\mathrm{cm}$ and the coefficients $b_{1}$ to $b_{4}$ given in Table 4.7. These equations are only valid for slab depths between $10 \mathrm{~cm}$ and $20 \mathrm{~cm}$.

$$
\begin{aligned}
& \varepsilon_{\theta, \text { subs }}=b_{1} h^{b_{2}}[\% o] \\
& \kappa_{\theta, \text { subs }}=b_{3} h^{b_{4}}[1 / \mathrm{km}] \\
& 10 \mathrm{~cm} \leq h \leq 20 \mathrm{~cm}
\end{aligned}
$$

Table 4.7: Coefficients for the determination of the substitute thermal loading

\begin{tabular}{|c|c|c|c|c|}
\hline & $b_{1}$ & $b_{2}$ & $b_{3}$ & $b_{4}$ \\
\hline R30 & 31.91 & -1.485 & 3867 & -1.924 \\
\hline R60 & 89.09 & -1.630 & 4976 & -1.845 \\
\hline R90 & 136.3 & -1.641 & 4889 & -1.744 \\
\hline
\end{tabular}




\subsubsection{Composite slabs}

The problem is more difficult for composite slabs. First of all, the thermal expansion is orthotropic due to different cross-sections in longitudinal and transverse direction. The substitute thermal strains and curvature therefore need to be determined separately for both directions. The thermal deformation furthermore is a two-dimensional problem. The temperature distribution in a transverse cross-section as shown in Figure 4.11 is the same in every cross-section along the slab but within the cross-section the temperature is different at any location. In order to determine the thermal forces $N_{\theta}$ and $M_{\theta}$ and the cross-section properties $E A, E S_{y}$ and $E I_{y}$, a two-dimensional integration over the cross-section area has to be performed. The integration over a polyangular area can only be done by numerical methods. The area has to be split into sub-areas, like finite elements, with constant temperatures. These sub-areas can be summed up to approximate the integration. The more sub-areas are considered, the more exact the result will be. The cross-section already needs to be modelled with finite elements to determine the temperature distribution. The mesh of the model can be input as fine as required and integration procedures are always included in finite element procedures. It is reasonable to also use the FE-program for the integrations, instead of reading out the temperatures and integrating with a spreadsheet software.

A longitudinal cross-section as marked in Figure 4.11 is rectangular at any location. The thermal forces and cross-section properties can be determined with a one-dimensional integration as described for full concrete slabs. Every cross-section has a different temperature distribution, depending on the location of the section. This means a beam has to be considered whose cross-section changes continuously, like a beam in shape of Figure 4.11. On such a system, it is difficult to analytically determine the location of the resulting neutral axis and the resulting bending stiffness $E I_{y}$.

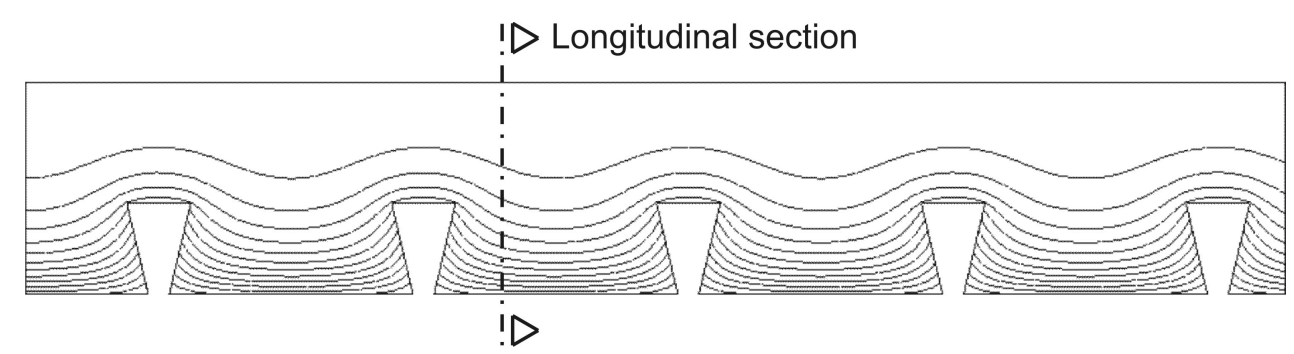

Fig. 4.11: Transverse section through composite slab with schematic isolines for temperature distribution

For these reasons, the substitute thermal loading for composite slabs is determined with finite element models in this work. The procedure will be explained on a Holorib slab in transverse direction. A two-dimensional beam with unit width in the y-direction as shown in Figure 4.12 is considered. It is clamped on one end in order to get a system with large deflections compared to its length. The larger the deflections are, the more exact are the results for the thermal curvature. In a first step, a thermal analysis is performed to get the temperature distribution in the beam as described in Section 4.1.2. The FE-model is changed from thermal to mechanical and the temperature field from the thermal analysis is applied to the mechanical model. In a second step, the location of the resulting neutral axis is identified as shown in Figure 4.12 above. For this calculation, the material in the model only has a temperature dependent Young's modulus, no thermal elongations. A force $F$ is applied at the end of the beam with a variable distance $a_{z}$ from the bottom of the slab. The distance $a_{z}$ is varied iteratively until no vertical deformation $w$ occurs. This distance $a_{z n}$ is then the location of the resulting neutral axis. For this procedure the fact is used, that a normal force, which acts in the neutral axis of a beam, causes no bending moments and 
therefore no deflection. In a third step, the force is removed and thermal elongation is included in the material model. With the resulting elongation $u$ and vertical deformation $w$ of the beam in the neutral axis as shown in Figure 4.12 below, the substitute thermal loadings can be calculated with following equations:

$$
\begin{aligned}
\varepsilon_{\theta, \text { subs }} & =\frac{u}{l} \\
\kappa_{\theta, \text { subs }} & =\frac{8 w}{(2 l)^{2}}
\end{aligned}
$$
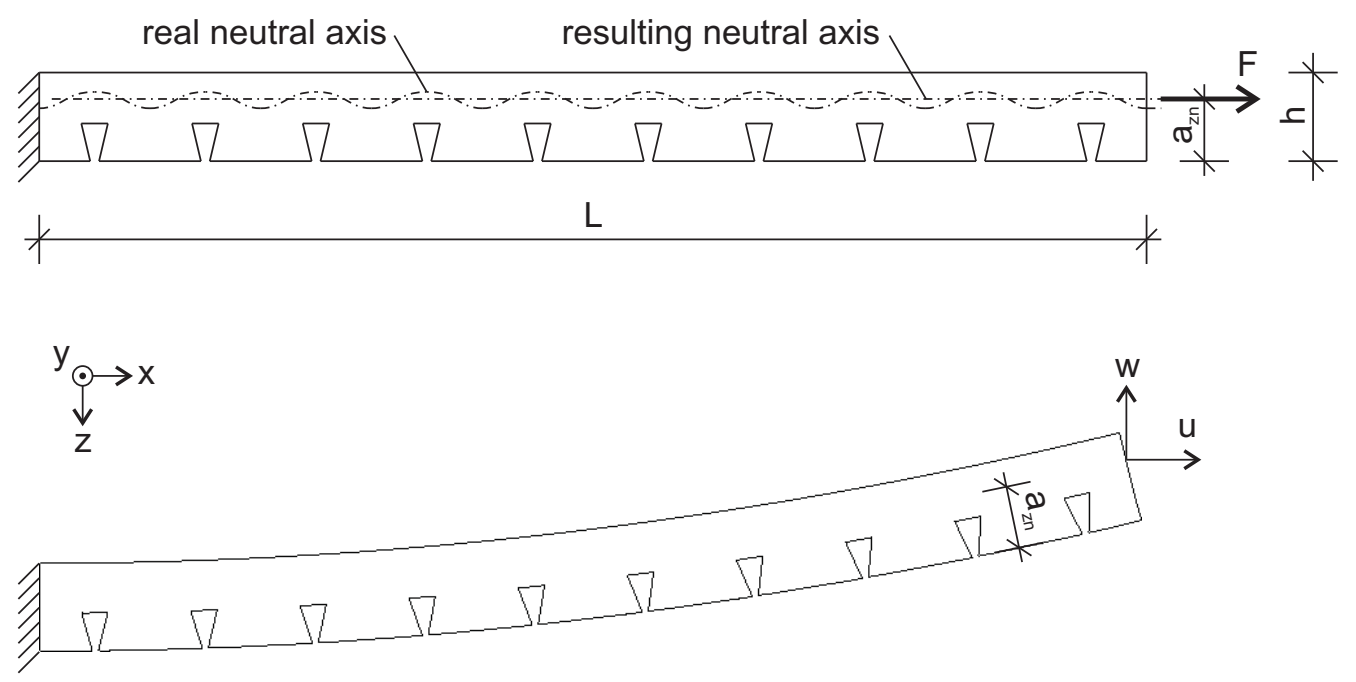

Fig. 4.12: Determination of location of neutral axis and thermal deformations in transverse direction

In the longitudinal direction, a system as shown in Figure 4.13 is used. Since the cross-section is not rectangular in the y-direction, no uniform width can be used and the model has to be three-dimensional. In order to reduce computing times, the symmetry of the system is used and only half of a rib is modelled. The horizontal displacements in the y-direction are fixed as shown in the cross-section in Figure 4.13 to ensure the same deformations as in a non-reduced system.

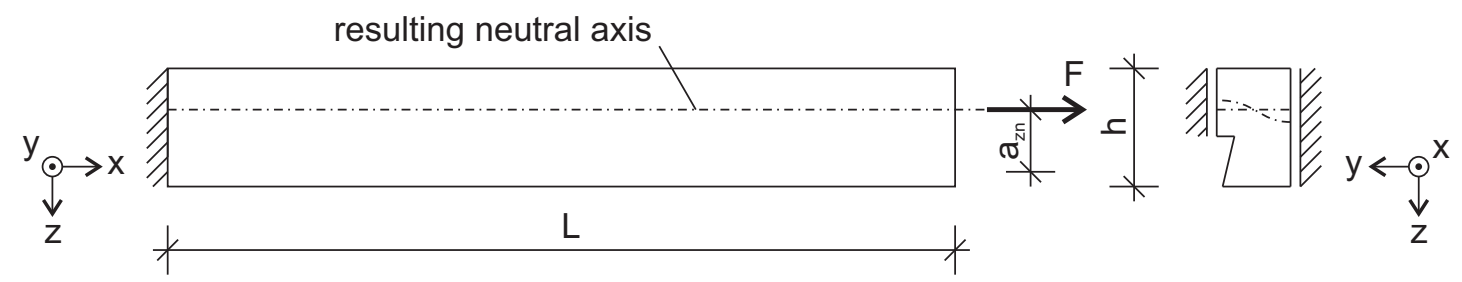

Fig. 4.13: System in longitudinal direction

The method described in this section allows the substitute thermal loading for any kind of cross-section to be determined. In order to demonstrate the method in this work, tables have been calculated for two common composite slabs: Holorib HR51 and Cofraplus 60. The three most important fire resistance classes R30 to R90, coming out of the standard fire curve, are chosen as for full concrete slabs. In Tables 4.8 and $4.9, h$ is the overall slab depth. Intermediate values can be interpolated. 
Table 4.8: Substitute thermal loading on composite slabs Holorib HR51

\begin{tabular}{|c|c|c|c|c|c|c|}
\hline Slab depth & \multicolumn{2}{|c|}{$\mathrm{R} 30$} & \multicolumn{2}{c|}{$\mathrm{R} 60$} & \multicolumn{2}{c|}{$\mathrm{R} 90$} \\
\hline $\mathrm{h}$ & $\varepsilon_{\theta, \text { subs }}$ & $\kappa_{\theta, \text { subs }}$ & $\varepsilon_{\theta, \text { subs }}$ & $\kappa_{\theta, \text { subs }}$ & $\varepsilon_{\theta, \text { subs }}$ & $\kappa_{\theta, \text { subs }}$ \\
\hline$[\mathrm{cm}]$ & {$[\%$ o $]$} & {$[1 / \mathrm{km}]$} & {$[\%$ o $]$} & {$[1 / \mathrm{km}]$} & {$[\%]$} & {$[1 / \mathrm{km}]$} \\
\hline \multicolumn{7}{|c|}{ longitudinal } \\
\hline 10 & 1.180 & 50.94 & 2.323 & 82.22 & 3.828 & 101.8 \\
\hline 12 & 0.857 & 36.50 & 1.708 & 57.75 & 2.546 & 74.82 \\
\hline 14 & 0.661 & 27.12 & 1.303 & 42.07 & 1.916 & 55.50 \\
\hline 16 & 0.536 & 20.72 & 1.023 & 32.29 & 1.539 & 41.97 \\
\hline 18 & 0.447 & 16.21 & 0.831 & 25.50 & 1.236 & 32.88 \\
\hline 20 & 0.383 & 12.95 & 0.692 & 20.56 & 1.019 & 26.54 \\
\hline \multicolumn{7}{|c|}{ transversal } \\
\hline 10 & 0.883 & 28.55 & 1.744 & 58.86 & 3.298 & 82.82 \\
\hline 12 & 0.607 & 20.11 & 1.311 & 40.05 & 2.166 & 59.72 \\
\hline 14 & 0.450 & 14.77 & 1.005 & 29.21 & 1.649 & 44.08 \\
\hline 16 & 0.348 & 11.17 & 0.781 & 22.66 & 1.322 & 33.19 \\
\hline 18 & 0.279 & 8.655 & 0.626 & 18.09 & 1.061 & 26.25 \\
\hline 20 & 0.229 & 6.854 & 0.516 & 14.71 & 0.868 & 21.39 \\
\hline
\end{tabular}

Table 4.9: Substitute thermal loading on composite slabs Cofraplus 60

\begin{tabular}{|c|c|c|c|c|c|c|}
\hline Slab depth & \multicolumn{2}{|c|}{$\mathrm{R} 30$} & \multicolumn{2}{c|}{$\mathrm{R} 60$} & \multicolumn{2}{c|}{$\mathrm{R} 90$} \\
\hline $\mathrm{h}$ & $\varepsilon_{\theta, \text { subs }}$ & $\kappa_{\theta, \text { subs }}$ & $\varepsilon_{\theta, \text { subs }}$ & $\kappa_{\theta, \text { subs }}$ & $\varepsilon_{\theta, \text { subs }}$ & $\kappa_{\theta, \text { subs }}$ \\
\hline$[\mathrm{cm}]$ & {$[\%$ o $]$} & {$[1 / \mathrm{km}]$} & {$[\%$ o $]$} & {$[1 / \mathrm{km}]$} & {$[\%$ ] $]$} & {$[1 / \mathrm{km}]$} \\
\hline \multicolumn{6}{|c|}{ longitudinal } \\
\hline 10 & 2.238 & 67.23 & 5.035 & 108.4 & 7.569 & 135.0 \\
\hline 12 & 1.511 & 52.25 & 3.078 & 88.83 & 4.831 & 110.5 \\
\hline 14 & 1.072 & 39.66 & 2.091 & 67.53 & 3.209 & 84.73 \\
\hline 16 & 0.808 & 30.46 & 1.595 & 49.85 & 2.259 & 64.83 \\
\hline 18 & 0.639 & 23.70 & 1.226 & 38.08 & 1.788 & 49.10 \\
\hline 20 & 0.526 & 18.71 & 0.974 & 29.98 & 1.433 & 38.01 \\
\hline \multicolumn{7}{|c|}{ transversal } \\
\hline 10 & 2.391 & 113.4 & 5.774 & 185.7 & 8.672 & 223.9 \\
\hline 12 & 1.452 & 67.48 & 3.329 & 112.1 & 5.290 & 143.0 \\
\hline 14 & 1.007 & 43.97 & 2.101 & 75.56 & 3.454 & 95.45 \\
\hline 16 & 0.729 & 31.29 & 1.578 & 52.51 & 2.335 & 69.18 \\
\hline 18 & 0.561 & 23.21 & 1.204 & 38.47 & 1.798 & 51.17 \\
\hline 20 & 0.449 & 17.74 & 0.943 & 29.65 & 1.444 & 38.70 \\
\hline
\end{tabular}

The substitute thermal loading of composite slabs depends on the geometry of the steel sheeting which can be described by five parameters $l_{1}$ to $l_{3}, h_{1}$ and $h_{2}$ as shown in Figure 4.14. The total depth $h=h_{1}+h_{2}$ is the governing parameter. A formula for calculating the substitute thermal loading must include the five parameters. A composite cross-section can be replaced by an equivalent rectangular cross-section with an effective thickness $h_{\text {eff }}$ according to DIN EN 1994-1-2, Annex D.4 [32]. The equation for calculating $h_{e f f}$ includes all five geometric parameters:

$$
h_{e f f}=h_{1}+0.5 h_{2}\left(\frac{l_{1}+l_{2}}{l_{1}+l_{3}}\right) \text { for } h_{2} / h_{1} \leq 1.5 \text { and } h_{1}>40 \mathrm{~mm}
$$



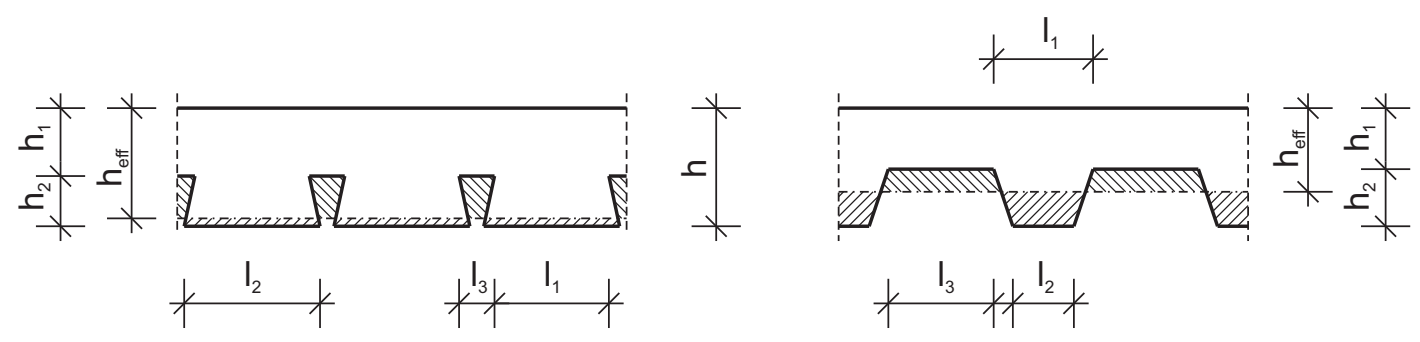

Fig. 4.14: Geometrical notation of steel deckings according to [32]

The area of a rectangular cross-section with the depth $h_{\text {eff }}$ is equal to the area of the original crosssection. If a composite slab is replaced by a full concrete slab with the depth $h_{e f f}$, the formulas determined in the previous section are a good approximation for the substitute thermal loading. As an example, the substitute thermal curvature $\kappa_{\theta, s u b s}$ of Holorib and Cofraplus 60 slabs in longitudinal direction are compared with full concrete slabs in Figure 4.15. It can be seen that the curves are in good agreement. In Equation (4.26) and (4.27) the slab depth $h$ simply has to be replaced by the effective thickness $h_{e f f}$ for calculating the substitute thermal loading of composite slabs in longitudinal direction.

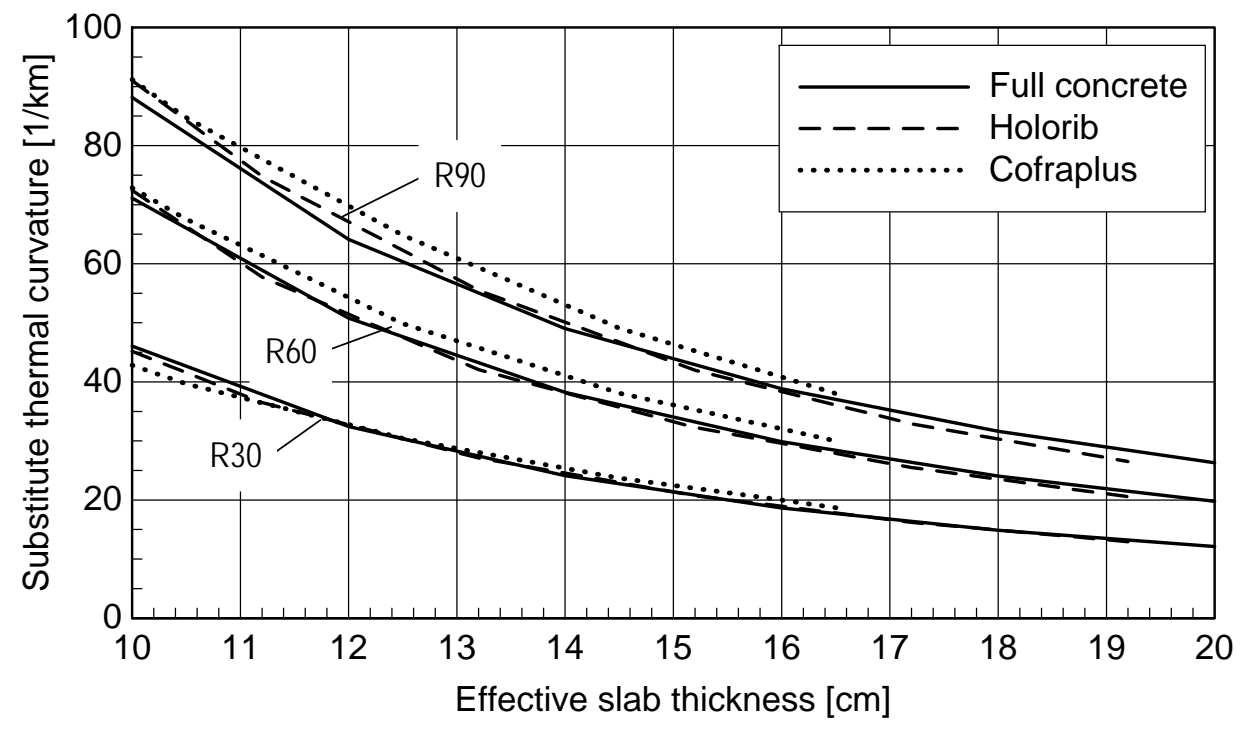

Fig. 4.15: Substitute thermal curvature in longitudinal direction $\kappa_{\theta, \text { subs }}$ of composite slabs as a function of the effective slab thickness $h_{\text {eff }}$

In transversal direction, the substitute thermal loading for Holorib slabs is slightly lower than the loading determined with the effective thickness, and for Cofraplus slabs it is slightly higher. In this case, a correction factor $\beta$ is included in Equation (4.26) and (4.27) which depends on the fire resistance class.

$$
\begin{aligned}
& \varepsilon_{\theta, \text { subs }}=\beta b_{1} h_{\text {eff }}^{b_{2}}[\% o] \\
& \kappa_{\theta, \text { subs }}=\beta b_{3} h_{\text {eff }}^{b_{4}}[1 / \mathrm{km}] \\
& 10 \mathrm{~cm} \leq h_{\text {eff }} \leq 20 \mathrm{~cm}
\end{aligned}
$$


Where:
$\beta=1.0$ in longitudinal direction;
$=c_{1} t+c_{2}$ in transversal direction;
$t \quad$ is the considered time in $[\mathrm{min}]$ of the fire resistance class;
$b_{1}$ to $b_{4} \quad$ are the coefficients given in Table 4.7;
$c_{1}$ and $c_{2}$ are the coefficients given in Table 4.10;

Table 4.10: Coefficients for the determination of the substitute thermal loading in transversal direction

\begin{tabular}{|l|c|c|}
\hline Profile type & $c_{1}$ & $c_{2}$ \\
\hline Re-entrant trough & 0.0038 & 0.47 \\
\hline Open trough & 0.0019 & 0.96 \\
\hline
\end{tabular}

In Figure 4.16 the substitute thermal curvatures $\kappa_{\theta, \text { subs }}$ of Holorib and Cofraplus 60 slabs of Table 4.8 and 4.9 are adjusted with the factor $\beta$. The curves are in good agreement with the curves of full concrete slabs. The coefficients $c_{1}$ and $c_{2}$ are determined with data of Holorib and Cofraplus 60 slabs. These could slightly vary for other profile types. A conservative simple assumption is therefore suggested that for slabs with re-entrant trough profile steel sheeting the substitute thermal loading in transversal direction is reduced by $20 \%$ and for open trough profile steel sheeting it is increased by $20 \%$.

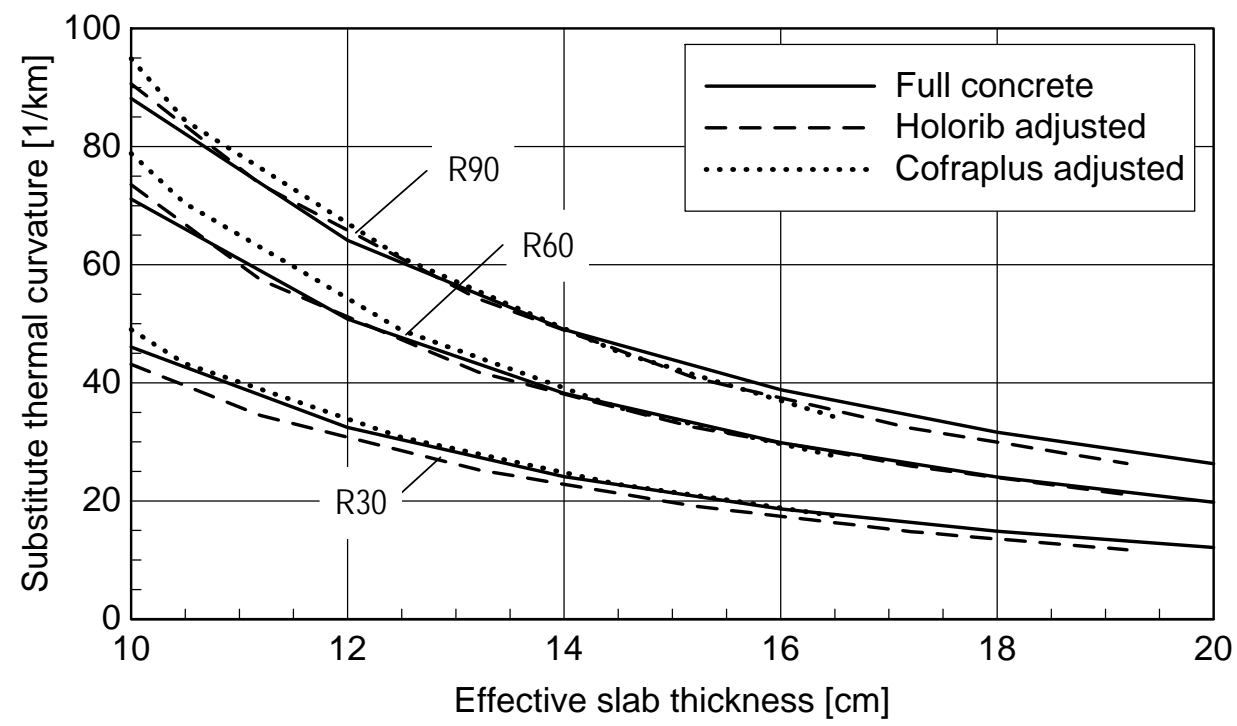

Fig. 4.16: Substitute thermal curvature in transversal direction $\kappa_{\theta, s u b s}$ of composite slabs as a function of the effective slab thickness $h_{e f f}$

To summarise, the substitute thermal loading of composite slabs can be calculated in good approximation with the formulas for full concrete slabs using the effective thickness. The values for the transversal direction need to be corrected by a factor $\beta$. Equation (4.32) and (4.33) for full concrete slabs can be used with the coefficients of Table 4.7. 


\subsubsection{Validation}

The approaches of the previous two Sections 4.2.2 and 4.2.3 can be used to validate each other. In the first example, a $1.50 \mathrm{~m}$ long beam is considered that is clamped at one end. It offers a rectangular crosssection with a depth of $12 \mathrm{~cm}$ and a unit width of $1.00 \mathrm{~m}$. The system and cross-section can be seen in Figure 4.17. The beam consists of concrete C25/30 with material properties according to DIN EN 19921-2 [27]. The values derived in Section 4.2.1 are used for the temperature dependent Young's modulus. The slab is exposed to the standard fire and the thermal analysis is performed with the heat transfer coefficients according to the Eurocodes as described in Section 4.1.

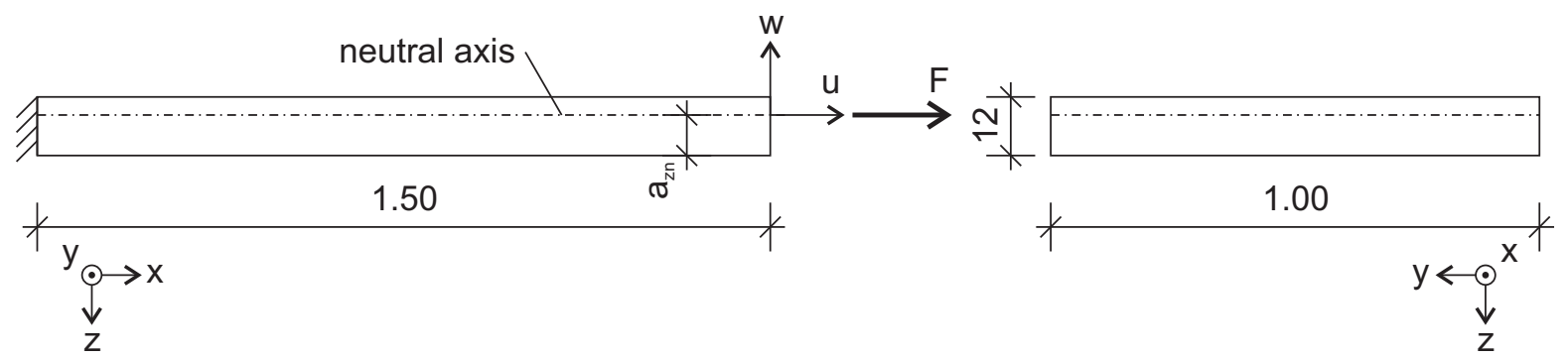

Fig. 4.17: System (left) and cross-section (right) of Example 4-1

The results are shown in Table 4.11. In a first FE-calculation, a force $F$ is applied to the beam without thermal strains. The force acts at the neutral axis whose distance from the bottom of the slab $a_{z n, a n a}$ was determined analytically. The simulated deformations in the z-direction $w_{F E A, F}$ are very small. This confirms the right location of the neutral axis. In a second analysis, no force is applied but thermal strains are taken into account. The deformations at the end of the slab $u_{F E A}$ and $w_{F E A}$ are measured in the neutral axis. They are compared with the deformations $u_{\text {ana }}$ and $w_{\text {ana }}$ which are determined from the analytically calculated substitute thermal strains $\varepsilon_{\theta, \text { subs,ana }}$ and curvatures $\kappa_{\theta, \text { subs,ana }}$ with following equations:

$$
\begin{aligned}
u_{\text {ana }} & =\varepsilon_{\theta, \text { subs,ana }} \cdot l \\
w_{\text {ana }} & =\frac{\kappa_{\theta, \text { subs,ana }} \cdot(2 l)^{2}}{8}
\end{aligned}
$$

The results are in good agreement. The differences are due to numerical tolerances. This confirms that both methods deliver the same results and both can be applied as required.

Table 4.11: Comparison of analytical method of Section 4.2.2 and FE-method of Section 4.2.3

\begin{tabular}{|l|c|c|c|c|}
\hline & & $\mathrm{R} 30$ & $\mathrm{R} 60$ & $\mathrm{R} 90$ \\
\hline$a_{\text {zn,ana }}$ & {$[\mathrm{m}]$} & 0.0763 & 0.0798 & 0.0826 \\
\hline$w_{F E A, F}$ & {$[\mathrm{~m}]$} & $2.86 \cdot 10^{-8}$ & $1.28 \cdot 10^{-7}$ & $1.74 \cdot 10^{-7}$ \\
\hline$\varepsilon_{\theta, \text { subs,ana }}$ & {$[-]$} & 0.000791 & 0.001576 & 0.002255 \\
\hline$\kappa_{\theta, \text { subs,ana }}$ & {$[1 / \mathrm{m}]$} & 0.03265 & 0.05058 & 0.06519 \\
\hline$u_{\text {ana }}$ & {$[\mathrm{mm}]$} & 1.187 & 2.364 & 3.383 \\
\hline$u_{F E A}$ & {$[\mathrm{~mm}]$} & 1.141 & 2.304 & 3.266 \\
\hline$w_{\text {ana }}$ & {$[\mathrm{mm}]$} & 36.73 & 56.90 & 73.34 \\
\hline$w_{F E A}$ & {$[\mathrm{~mm}]$} & 37.09 & 56.70 & 72.15 \\
\hline
\end{tabular}

In Example 4-2 the Systems shown in Figure 4.18 are considered. A combined thermal-mechanical- 
analysis is performed on a very short beam. The software Ansys APDL, Release 12.1 is used for this example. The input file can be found in Annex B.1. The material properties and heat transfer coefficients are again chosen according to the Eurocodes. Thermal strains are taken into account. The beam is heated on the bottom surface by the standard fire for thirty minutes. In Example 4-2a, it is clamped at both ends in order to provide horizontal restraint. The stresses in the longitudinal direction in the central cross-section are shown in Figure 4.19 on the left. These are non-linear thermal stresses $\sigma_{\text {nonl }}(\theta)$ that have been introduced in Section 4.2.1, Figure 4.8. In Example 4-2b, the beam is only clamped on one end as shown in Figure 4.18 on the right hand side. Here the beam can deform freely and no restraint occurs. The stresses in the central cross-section can be seen in Figure 4.19 on the right. These are the self-equilibrating stresses $\sigma_{\theta, \text { self }}$ that have been explained in Section 4.2.1, as well. This example confirms qualitatively the assumptions of Section 4.2.1.
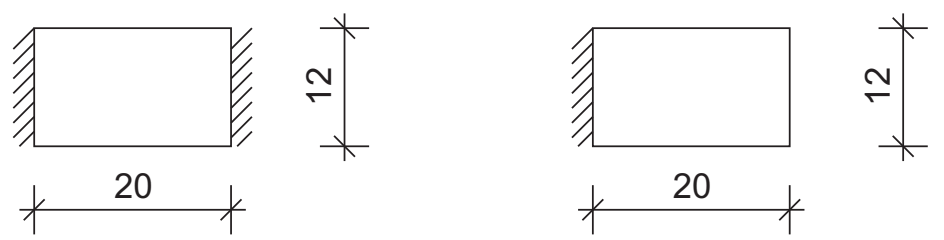

Fig. 4.18: Systems of Example 4-2a (left) and b (right)
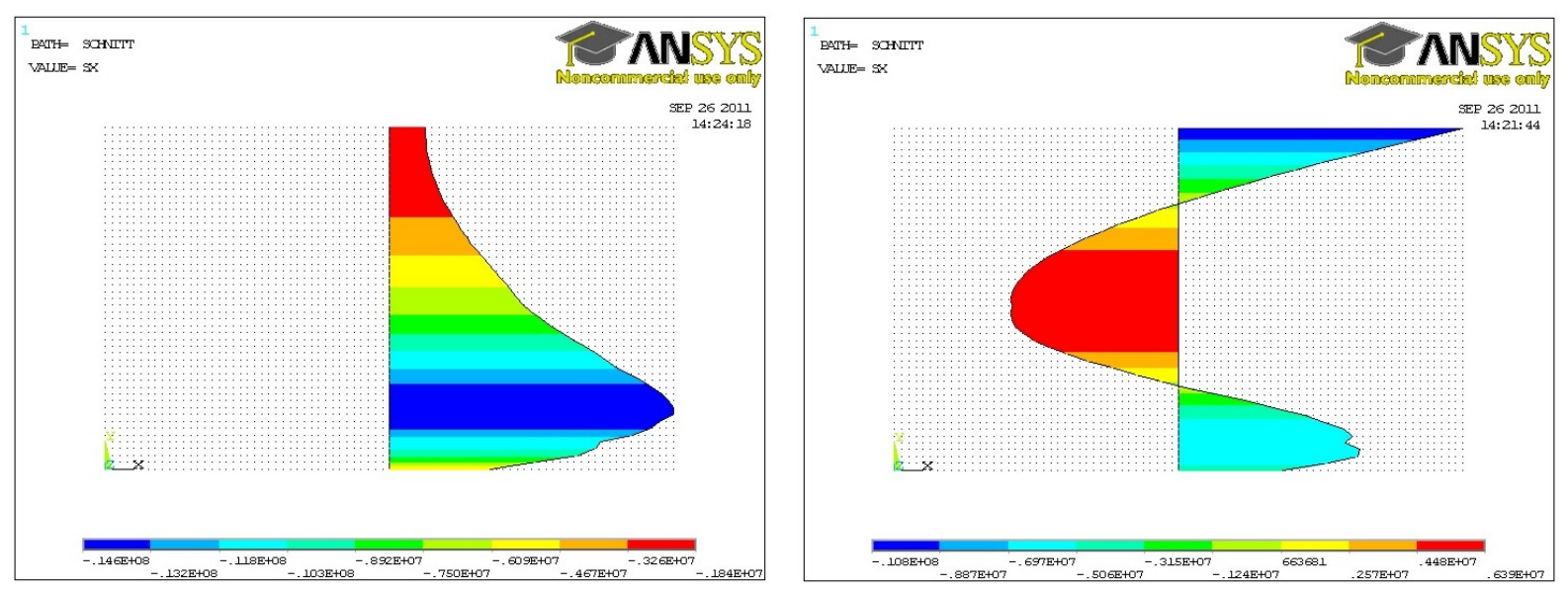

Fig. 4.19: Stresses in longitudinal direction in central cross-section of Example 4-2a (left) and b (right)

\subsection{Substitute thermal loading on beams}

The temperature distribution in the steel section of composite beams can be determined, like in slabs, by advanced calculation models using finite element analysis. In Eurocode 4 [32] there are also simplified calculation models for unprotected and insulated steel beams which deliver reasonable results. These are much faster in application and, therefore, no numerical simulations are required for the thermal analysis of the steel beams. The method in the Eurocode enables the user to calculate a different temperature in the flanges and the web of beams. Within these parts the temperature is assumed to be uniform. With the temperature dependent thermal strain of steel according to DIN EN 1994-1-2, 3.3.1 [32] the elongation of every part can be determined. This would mean, however, that every flange and web had to be modelled separately in a finite element simulation when a mechanical analysis was performed. The following 
simplifications are therefore made in this work. A uniform mean temperature is used for the whole steel section. This allows the whole steel beam to be modelled with beam elements. For the simulations of the fire tests, where different temperatures are measured in the flanges and the web, a mean temperature $\theta_{m}$ is calculated by using following equation:

$$
\theta_{m}=\frac{\sum A_{i} \theta_{i}}{\sum A_{i}}
$$

For the design procedure of new structures the methods in DIN EN 1993-1-2, 4.2 .5 [29] are used. With the formulas given there, a uniform temperature of a steel section can be calculated which is exposed to a fire on three sides and protected by a concrete slab on the fourth side.

The following Example 4-3 shall justify the simplifications. A composite beam is considered as shown in Figure 4.20. It is subjected to the standard fire from below for thirty minutes. The steel beam is unprotected. For the mechanical analysis the finite element software Sofistik, Version 11.17 is used. It has been developed particularly for the requirements of structural engineering and is chosen for this work due to its useful post-processor. The input file of this example can be found in Annex B.2. The concrete slab is modelled with four shell elements in the cross-section and the steel beam with five shell elements. Two additional nodes are input at the intersection of the beam and the slab. Each of the two nodes is rigidly coupled with the slab above or the beam below. At the two nodes themselves only the displacements are coupled to each other, no rotations. Linear elastic material is used for both, concrete and steel. The Young's modulus of the concrete slab is simplified set uniformly according to DIN EN 1992-1-1 [26] since the stiffness of the slab is irrelevant at this example. The Young's modulus of the steel is set according to its temperature. The slab is loaded with a substitute thermal loading of $\varepsilon_{\theta, \text { subs }}=0.791 \%$ and $\kappa_{\theta, \text { subs }}=32.65 \mathrm{~km}^{-1}$ out of Table 4.6 .

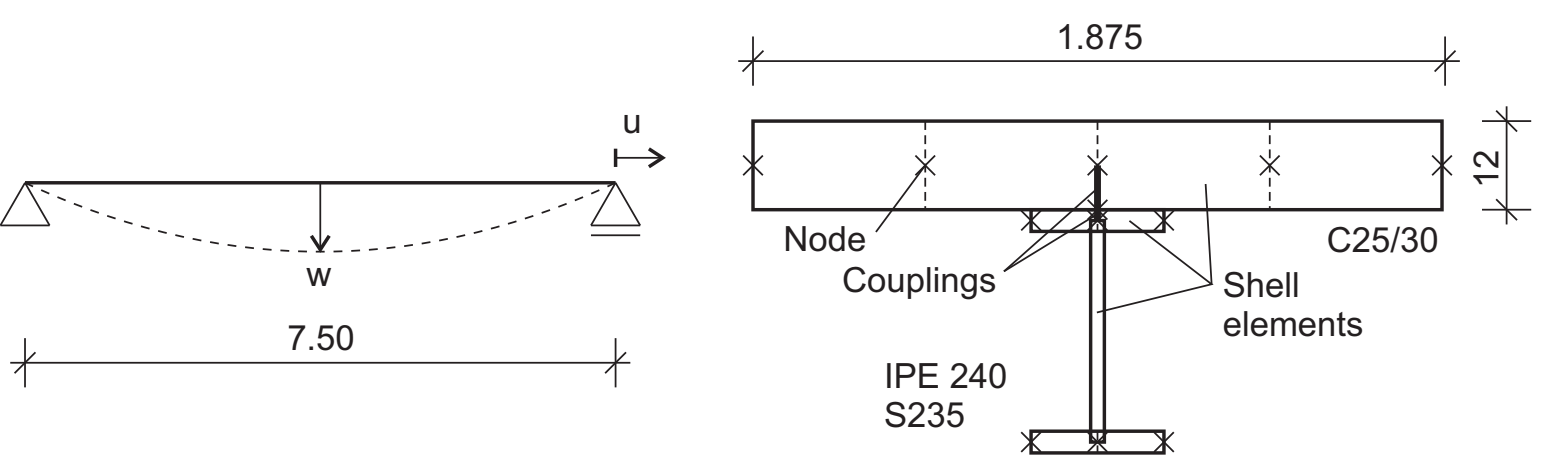

Fig. 4.20: System (left) and cross-section (right) of Example 4-3: composite beam

In Example 4-3a the temperatures in the flanges and the web are calculated with the simplified method of DIN EN 1994-1-2, 4.3.4.2.2 [32]. The resulting temperatures $\theta$, Young's moduli $E_{\theta}$ and thermal strains $\varepsilon_{\theta}$ for each part are shown in Table 4.12. It can be seen that the temperatures and, hence, the thermal strains are very close together. In Example $4-3 \mathrm{~b}$ a mean temperature is used, calculated with Equation (4.37).

$$
\theta_{m}=\frac{\sum A_{i} \theta_{i}}{\sum A_{i}}=\frac{1176(741+814)+1366 \cdot 831}{2 \cdot 1176+1366}=797^{\circ} \mathrm{C}
$$

Example 4-3c is performed with the simplified method of Eurocode 3 [29] where a uniform temper- 
Table 4.12: Material properties of steel beam in Example 4-3

\begin{tabular}{|l|c|c|c|}
\hline & $\theta$ & $E_{\theta}$ & $\varepsilon_{\theta}$ \\
\hline & {$\left[{ }^{\circ} \mathrm{C}\right]$} & {$[\mathrm{MPa}]$} & {$[\%$ o $]$} \\
\hline \multicolumn{4}{|c|}{ Example 4-3a } \\
\hline Upper flange & 741 & 23940 & 10.85 \\
\hline Web & 831 & 17430 & 11.00 \\
\hline Lower flange & 814 & 18270 & 11.00 \\
\hline \multicolumn{4}{|c|}{ Example 4-3b } \\
\hline Uniform & 797 & 19150 & 11.00 \\
\hline \multicolumn{4}{|c|}{ Example 4-3c } \\
\hline Uniform & 808 & 18520 & 11.00 \\
\hline
\end{tabular}

ature is calculated directly for the whole beam. In Table 4.12 it can be seen that the temperatures of Example 4-3b and c are very close together. The resulting deflection $w$ at midspan and the elongation $u$ of all three examples are compared in Table 4.13. The differences are negligible. This confirms that a uniform temperature in the steel beams can be assumed.

Table 4.13: Comparison of results of Example 4-3

\begin{tabular}{|c|c|c|}
\hline Example & $w$ & $u$ \\
\hline & {$[\mathrm{mm}]$} & {$[\mathrm{mm}]$} \\
\hline $4-3 \mathrm{a}$ & 249.5 & 6.31 \\
\hline $4-3 \mathrm{~b}$ & 248.3 & 6.27 \\
\hline $4-3 \mathrm{c}$ & 247.9 & 6.26 \\
\hline
\end{tabular}

The determination of the temperature in protected beams is not always possible. For some protection systems, like intumescent coatings, no simplified calculation models are available so far. First approaches have been made to simulate the behaviour of reactive coatings and to perform a thermal analysis with finite elements, for example in [42]. These approaches are at the beginning of research however and no reliable results can be produced at the moment. Fire protection systems usually have to confirm their applicability with fire tests. In Germany tests on reactive coatings have to follow DIN EN 13381-8 [20]. Beams and columns are mechanically loaded and exposed to the standard fire curve. The temperatures at different locations in the steel sections are recorded continuously. Results of the tests are published in separate tables for different fire resistance classes (R30, R60 etc.) that contain a required coat thickness for different section factors and critical temperatures. The critical temperature is not specified further in DIN EN 13381-8 [20]. It just says that the determined critical temperatures can be used for the design of steel members according to DIN EN 1993-1-2 and DIN EN 1994-1-2. The critical temperature in these codes depends on the load applied to the member. It varies from $1100^{\circ} \mathrm{C}$ for unloaded members and can be up to $20^{\circ} \mathrm{C}$ when stability has to be taken into account. This means that the critical temperature has to be determined for every beam and a corresponding coat thickness has to be applied. For manufacturing reasons the coat thickness is usually not varied too much within one building. The true temperatures can therefore not be reliably predicted.

The worst case of the standard fire that leads to the highest temperatures in the beams is not necessarily the worst case for the slab. This is in contrast to the slabs. There the standard fire induces very large temperature gradients. The larger the gradients are, the larger are the restraint forces in the slab. If the 
temperature in a beam is very high, it becomes very soft and provides a very soft support for the slab. A softer support reduces the hogging moments in the slab above the beam. Whereas, if the beam stays cold and stiff, the hogging moments in the slab above the beam are larger. The softer beam in turn increases the forces in the middle of the slab. To summarise, it is not obvious which temperature in the beams leads to the worst case for the slab.

The behaviour of the beams is therefore considered at limiting temperatures in the design method of this work. One calculation is performed with the lower limit of the beam temperature of $20^{\circ} \mathrm{C}$. This leads to the largest hogging moments in the slab above the beam. For a second calculation the highest possible temperature is used in order to get the highest forces in the middle of the slab. The upper limit can either be the critical temperature of the beam or the predicted maximum temperature that will be reached with the applied coat thickness. If both values are not known, the following simplification can be used for the upper limit. It originates from DIN 4102-4 [19] where a simplified critical temperature of $500^{\circ} \mathrm{C}$ was given. This temperature resulted from a maximum possible load level of $58 \%$ of beams where stability failure is excluded. This can be adopted to the regulations in the Eurocodes. A simplified maximum utilization factor of $\mu_{0}=0.65$ is given in DIN EN 1993-1-2, 4.2 .4 [29]. It is valid if both partial factors for ambient temperature $\gamma_{M 0}$ and for the fire situation $\gamma_{M, f i}$ are set to 1.0 in the national annex of the Eurocode. With this utilization factor a critical temperature of $540^{\circ} \mathrm{C}$ can be determined with the equation of DIN EN 1993-1-2, 4.2.4 [29]:

$$
\theta_{a, c r}=39.19 \ln \left[\frac{1}{0.9674 \mu_{0}^{3.833}}-1\right]+482=540^{\circ} \mathrm{C}
$$

With the design method of this work it is not very time consuming to consider different temperature levels since they are applied to the system as a load case. It is very easy to consider different load cases in order to find out the worst load scenario for each part of the structure. This method enables a procedure for designing slabs for the fire case which is standard for ambient temperature and engineers are used to. 


\section{Mechanical analysis}

The objective of this chapter is to simplify the mechanical analysis and to give recommendations for setting up the finite element model. Simplifications are primarily made to the material laws. These nonlinearities are the main reasons for the large computing times and convergence problems of advanced finite element models. The non-linear materials are replaced by linear material laws with reduced stiffness. Geometrical non-linearities cannot be replaced. Large deformations need to be taken into account in order to activate membrane forces.

\subsection{Material non-linearities}

In this section the way in which material non-linearities are generally included in finite element routines, analytical models and simplified calculation methods is explained. This theoretical background is necessary in order to understand how the material laws can be simplified.

\subsubsection{Finite element formulations}

Can Tesar developed the finite element program SlabFem within his dissertation [51] which is specialised for simulating membrane action. He describes in detail how a finite element routine and in particular his software takes the material non-linearities into account. Here a brief summary will be given. Generally, a finite element problem is solved with following equation:

$$
\mathbf{F}=\mathbf{K} \mathbf{u}
$$

Where $\mathbf{F}$ is a vector of the forces on the nodes, $\mathbf{u}$ is a vector of the node displacements and $\mathbf{K}$ is the stiffness matrix. The stiffness matrix can be written in more detail as follows:

$$
\mathbf{K}=\left[\begin{array}{ll}
\mathbf{k}_{u u} & \mathbf{k}_{u w} \\
\mathbf{k}_{w u} & \mathbf{k}_{w w}
\end{array}\right]
$$

For a finite element which includes membrane forces, the sub-matrices $\mathbf{k}_{i j}$ can be written as:

$$
\begin{aligned}
\mathbf{k}_{u u} & =\int E A \mathbf{b}_{u} \mathbf{b}_{u}^{T} d x \\
\mathbf{k}_{u w} & =\mathbf{k}_{w u}=\int E A \mathbf{b}_{w}^{T} \mathbf{w} \mathbf{b}_{u} \mathbf{b}_{w}+E S \mathbf{b}_{u} \mathbf{c}^{T} d x \\
\mathbf{k}_{w w} & =\int\left(E I \mathbf{c c}^{T}+E A\left(\mathbf{b}_{w}^{T} \mathbf{w}\right)^{2}+N\right) \mathbf{b}_{w} \mathbf{b}_{w}^{T}+E S\left(\mathbf{b}_{w}^{T} \mathbf{w}\right)\left(\mathbf{b}_{w} \mathbf{c}^{T}+\mathbf{c b}_{w}^{T}\right) d x
\end{aligned}
$$

The vectors $\mathbf{b}$ contain shape functions, $\mathbf{c}$ derivation operators and $\mathbf{w}$ displacements. They are not important for explaining the context here and will be not focused in more detail. The important point is that Equations (5.3) to (5.5) include the cross-section stiffness $E A, E S$ and $E I$. These values are determined 
in SlabFem and Vulcan on a layered cross-section as shown in Figure 5.1 with following equations:

$$
\begin{aligned}
& E A=\int E(z) d A \approx \sum E_{i} x_{i} \Delta z_{i} \\
& E S=\int E(z) z d A \approx \sum E_{i} x_{i} \Delta z_{i} z_{i} \\
& E I=\int E(z) z^{2} d A \approx \sum E_{i} x_{i} \Delta z_{i} z_{i}^{2}
\end{aligned}
$$

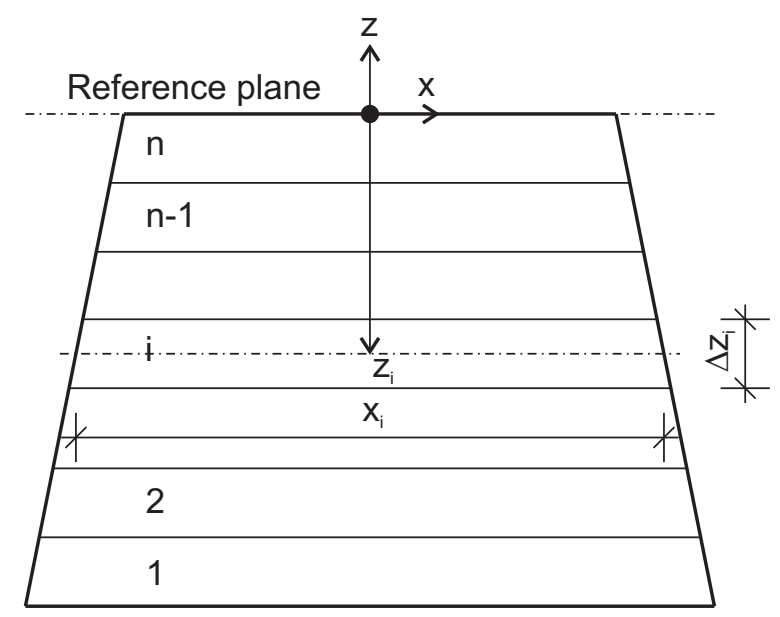

Fig. 5.1: Layered element according to Tesar [51]

These cross-section properties are determined in a similar way to in Section 4.2.2. Each layer $i$ has a certain stiffness $E_{i}$. This stiffness can be compared with the tangent modulus of the related nonlinear stress-strain curve. It depends on two factors: the temperature of the layer $\theta_{i}$ and the strain rate $\varepsilon_{i}$. The higher the temperature and strain are, the lower is the stiffness as shown in Figure 5.2. As a result, the material non-linearities have only one effect on a finite element formulation: they reduce the stiffness of the element expressed through its stiffness matrix. If the resulting stiffness is known before the simulation, it can be reduced simply by reducing the Young's modulus when the model is set up.

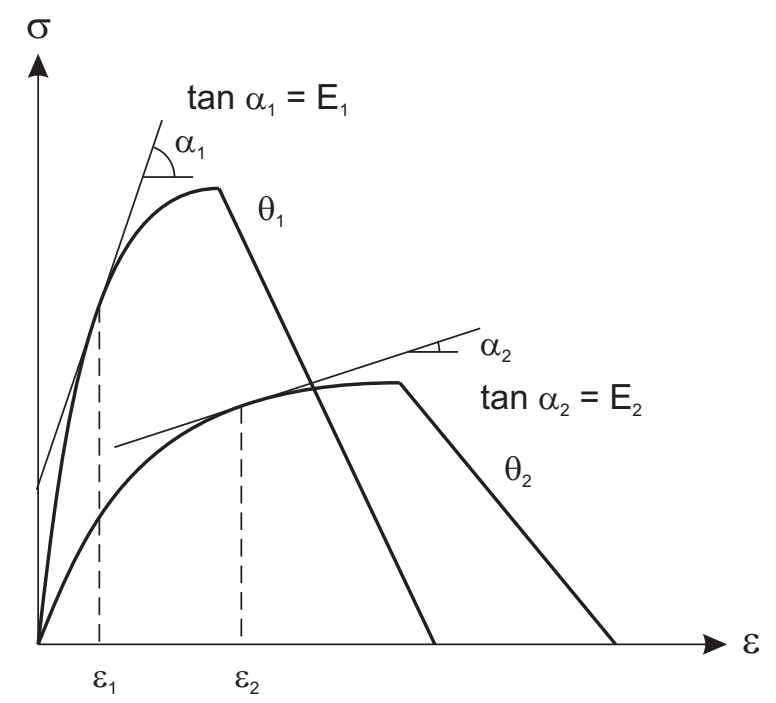

Fig. 5.2: Stiffness as a function of temperature and strain rate 


\subsubsection{Analytical models for ambient temperature}

Analytical models are hand calculation methods which are commonly used at ambient temperature. In these models only the time just before failure of a structure is considered, the so-called ultimate limit state (ULS). The times in between, from the beginning of the loading until failure, are not usually considered contrary to advanced numerical simulations. A structure is split into a system of several members, the internal forces are determined on this system and the load bearing resistance is calculated with crosssection design methods on every member. In these analytical models material non-linearities are taken into account in two ways; plastic resistance of the cross-sections and plastic global analysis to determine the internal forces. For a cross-section design, where no material non-linearities are taken into account, a cross-section reaches its load-bearing capacity when the yield strength of the material is reached anywhere in the section. The cross-section stiffness properties EA, ES and EI do not change in this method. This method is traditionally called elastic-elastic in Germany. It means that both the internal forces and the cross-section resistance are determined elastically.

Plastic cross-section design assumes that parts in the section which have reached the material strength start to yield and stress remains constant while strains continue to rise. The forces are distributed to parts of the section which can still bear forces. The ultimate limit state is reached when every part in the section reaches the material strength. The stiffness of the cross-section reduces as soon as the first part reaches yield strength. A so-called plastic hinge forms at this location of the structure. This means that no further forces can be borne and the structure can deform without resistance like a hinge. The loadbearing capacity of the whole structure is reached in this method when the first plastic hinge develops at any location. This method is called elastic-plastic. It means that the internal forces are determined elastically and the cross-section resistance plastically.

For a plastic global analysis which is called plastic-plastic, both the internal forces and the crosssection resistance are determined plastically. When a cross-section forms a plastic hinge somewhere in the structure, the loads are distributed to parts of the structure which still can bear more load. The load-bearing capacity of the whole structure is reached when as many plastic hinges have formed as that the structure becomes kinematic. This plastic global analysis is - in a slightly different way - generally used in hand calculation models. Many connections are considered as perfect hinges although these can always transfer a certain bending moment. This assumption is appropriate since at the ultimate limit state plastic hinges occur at these connections and the load is transferred to less loaded parts of the structure. In this method it is conservatively assumed that the plastic hinges at the connections have entirely no stiffness.

During the design of reinforced concrete and composite structures the complex material behaviour of concrete and plastic global analysis is taken into account as follows. Parts of the structure, where the concrete is expected to crack, are considered with a lower stiffness than the uncracked parts. This is mainly at intermediate supports where hogging moments occur. The hogging moments are reduced and the load is transferred to parts of the structure which are able to bear more loads. In Eurocode 2 [26] and 4 [31] a simplified form of this procedure can be found. It is called limited redistribution and allows reducing the hogging moments by an arbitrary chosen amount of up to $30 \%$. This means that the stiffness of the structure is chosen relatively arbitrarily in order to distribute the forces in a way that the required reinforcement is optimised at the ultimate limit state.

These explanations show that it is very common in classical design methods for ambient temperature to consider only the ultimate limit state of a structure. The stiffness is estimated more or less arbitrarily, 
the internal forces are determined with linear elastic material laws and every cross-section is designed for its forces. Unlike current fire case design it is unusual for ambient temperature that advanced calculation methods with complex finite element models and non-linear material laws are used which consider every load step from the beginning of the loading until failure. The design procedure presented in this work therefore adopts the ambient temperature approaches for composite slab systems in case of fire. A stiffness of the structure is determined that allows a linear elastic analysis and a simple cross-section design at the ultimate limit state.

\subsection{Modelling of the slabs}

\subsubsection{Geometry and elements}

Different options are available for the modelling of slabs with finite elements. The internal forces of a plate under large deformations can be seen in Figure 5.3. The simplest option is setting up a grid of beams in longitudinal and transversal direction and coupling the deformations at the intersection points. With this approach an orthotropic behaviour of a slab is easy to model since different cross-sections of the beams can be chosen in both directions. Important aspects are neglected, however, including Poisson effects which cannot be taken into account with beam grids as well as in-plane shear since the shear forces $n_{x y}=n_{y x}$ in Figure 5.3 cannot occur.
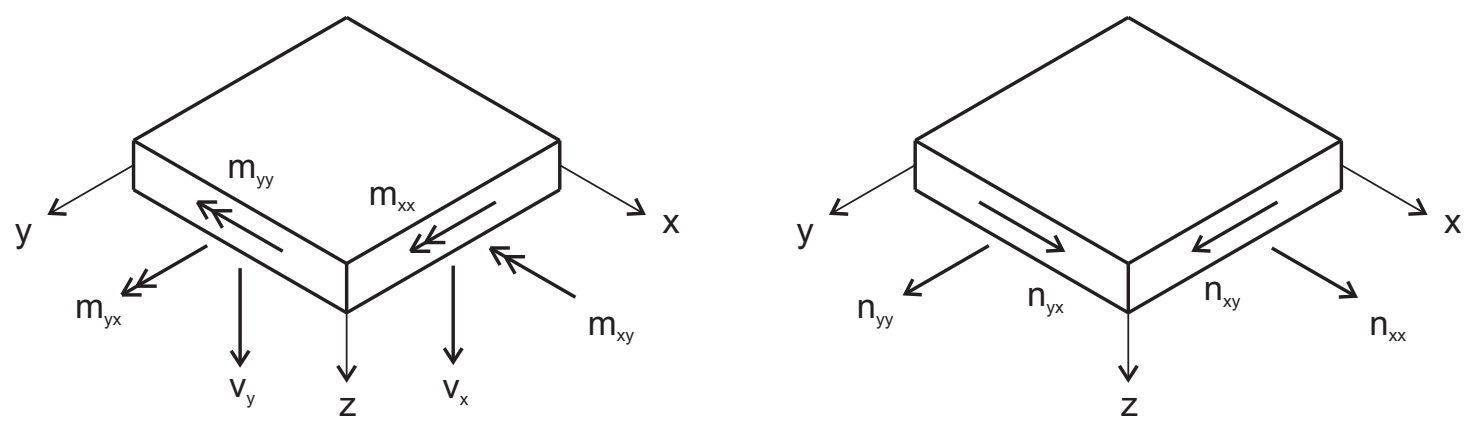

Fig. 5.3: Internal forces in a plate under large deformations

Slabs with small deformations under ambient temperature are usually modelled with plate elements. These can take into account all forces of the left hand side of Figure 5.3, the in-plane shear forces $n_{x y}=n_{y x}$ of the right hand side and Poisson effects. Plate elements follow two different theories: Kirchhoff theory for thin plates or Reissner-Mindlin theory for thick plates. The limit between a thick and a thin plate lies about at $10 \%$ of the shorter span of the slab [3]. The Reissner-Mindlin theory is based on the Kirchhoff theory but in addition takes into account the shear deformation of the slab. Plate elements deliver reasonable results as long as the deformations remain smaller than about $20 \%$ of the plate thickness [3].

If larger deformations occur, shell elements need to be used. These include membrane forces $n_{x x}$ and $n_{y y}$ in addition to the bending moments of Figure 5.3. Shell elements can also follow either the Kirchhoff or the Reissner-Mindlin theory.

If a slab is modelled with any of these elements above at elevated temperatures, there is always the problem that only the mechanical analysis can be performed with this model but not the thermal analysis. Ordinary shell elements, furthermore, can only include a uniform material, temperature and stiffness. In 
order to get a temperature distribution over the slab depth in a thermal analysis, at least two or more elements are required over the depth. This means that, in a sense, for a coupled thermal mechanical analysis a slab needs to be modelled three-dimensionally with volume elements. The computing time of a finite element solution, however, increases quadratically with every additional dimension, and transferring the node forces of the results into bending moments, shear and membrane forces is very time-consuming. The simplified programs Vulcan and SlabFem therefore use a so-called layered shell element. A shell element is divided into several layers as shown in Figure 5.1. Each layer can be given a certain temperature and the element determines a resulting stiffness as explained in Section 5.1.1. These layered shell elements allow a slab to be modelled two-dimensionally. The temperature distribution over the slab depth, however, has to be determined in a separate thermal analysis and the temperature field has to be transferred manually from the thermal to the mechanical model.

In the method of this thesis simple shell elements are used. In the software Sofistik, which is used in this work, only elements which follow the Reissner-Mindlin theory are implemented. This is the more exact theory but the Kirchhoff theory would also be sufficient since the depth of composite slabs is generally very small. The temperature distribution is taken into account in two ways. The thermal expansion is considered with a substitute thermal loading as derived in Chapter 4 and the reduced stiffness due to elevated temperatures with a resulting stiffness as explained in the following sections.

The geometry of slabs to be modelled with the presented method does not need to be limited. Every aspect ratio of slab panels can be modelled as well as non-rectangular slabs. This method is therefore not limited in its application spectrum in terms of geometrical issues.

\subsubsection{Influence of stiffness}

In the previous sections it was highlighted that material non-linearities only influence the stiffness of the slab, and that in classical design methods for ambient temperature the stiffness is chosen relatively arbitrarily. The influence that the stiffness has on composite slab systems under elevated temperatures is shown in Example 5-1. A single full concrete slab panel with a depth of $10 \mathrm{~cm}$ is considered as shown in Figure 5.4. The slab is modelled with simple shell elements. It is supported by protected edge beams which are modelled with beam elements and coupled eccentrically to the slab. The modelling of the beams is discussed in Section 5.3. The unprotected secondary beams are not modelled in this example. The slab is simply supported with supports on all four corners.

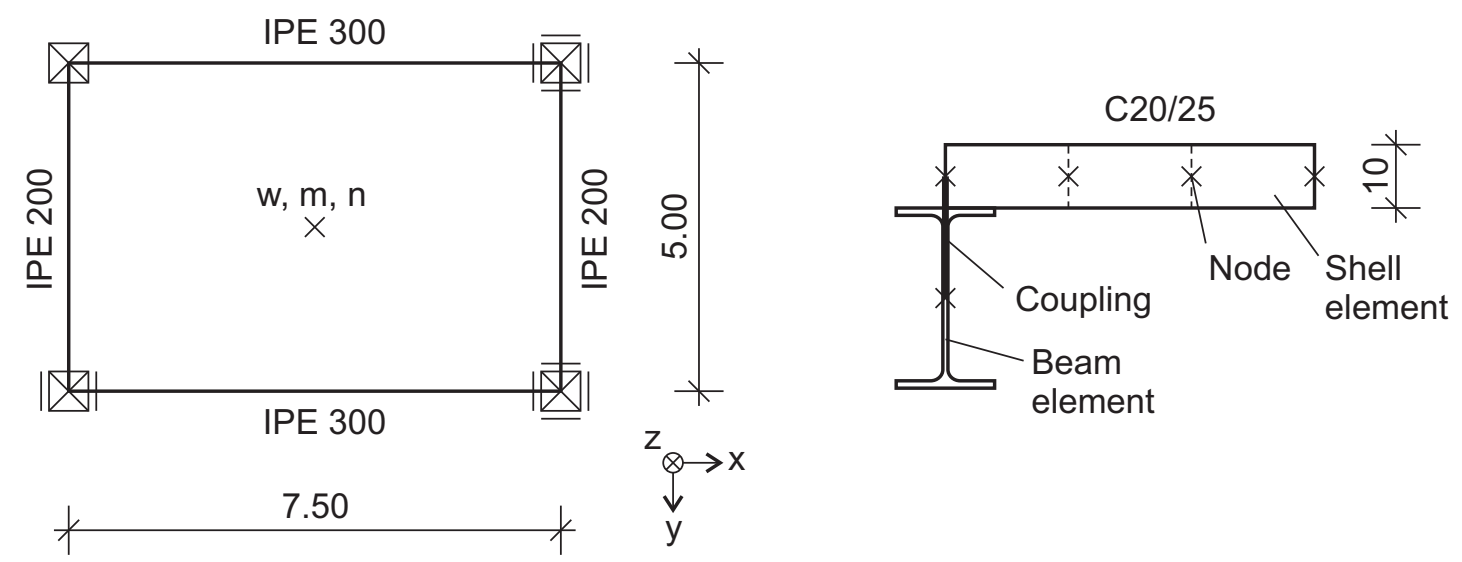

Fig. 5.4: Plan view (left) and cross-section (right) of Example 5-1 
The slab is loaded with a substitute thermal loading for R90 of $\varepsilon_{\theta, \text { subs }}=3.169 \%$ and $\kappa_{\theta, \text { subs }}=$ $87.41 \mathrm{~km}^{-1}$ according to Table 4.6. The temperature in the beams is assumed to reach $540^{\circ} \mathrm{C}$ as explained in Section 4.3. This is taken into account with a substitute thermal loading on the beams of $\varepsilon_{\theta, \text { subs }}=7.405 \%$ and a reduced Young's modulus of $E_{\theta}=101640 \mathrm{~N} / \mathrm{mm}^{2}$ according to Eurocode 3 [29] or 4 [32]. A mechanical distributed load of $5.34 \mathrm{kN} / \mathrm{m}^{2}$ is applied. This loading consists of the selfweight of the slab, an additional dead load of $2.0 \mathrm{kN} / \mathrm{m}^{2}$, a live load of $2.0 \mathrm{kN} / \mathrm{m}^{2}$ and an additional load for movable partitions of $0.80 \mathrm{kN} / \mathrm{m}^{2}$. These loads are combined with the combination of actions for accidental design situations according to DIN EN 1990, 6.4.3.3 [21] with a factor for the quasi-permanent value of a variable action $\psi_{2}=0.3$. This value is taken from the German national annex of DIN EN 1990 [22].

$$
q_{E d, f i}=g_{k}+\psi_{2} q_{k}=25 \cdot 0.10+2.0+0.3(2.0+0.8)=5.34 \mathrm{kN} / \mathrm{m}^{2}
$$

Linear elastic material laws are assumed. Geometrical non-linearities are taken into account to be able to activate membrane forces. This example is modelled with the software Sofistik. The input file can be found in Appendix B.3. Although a geometrically non-linear system has to be solved, the computing time of this example remains below one minute. This very short computing time illustrates one of the advantages of the presented method.

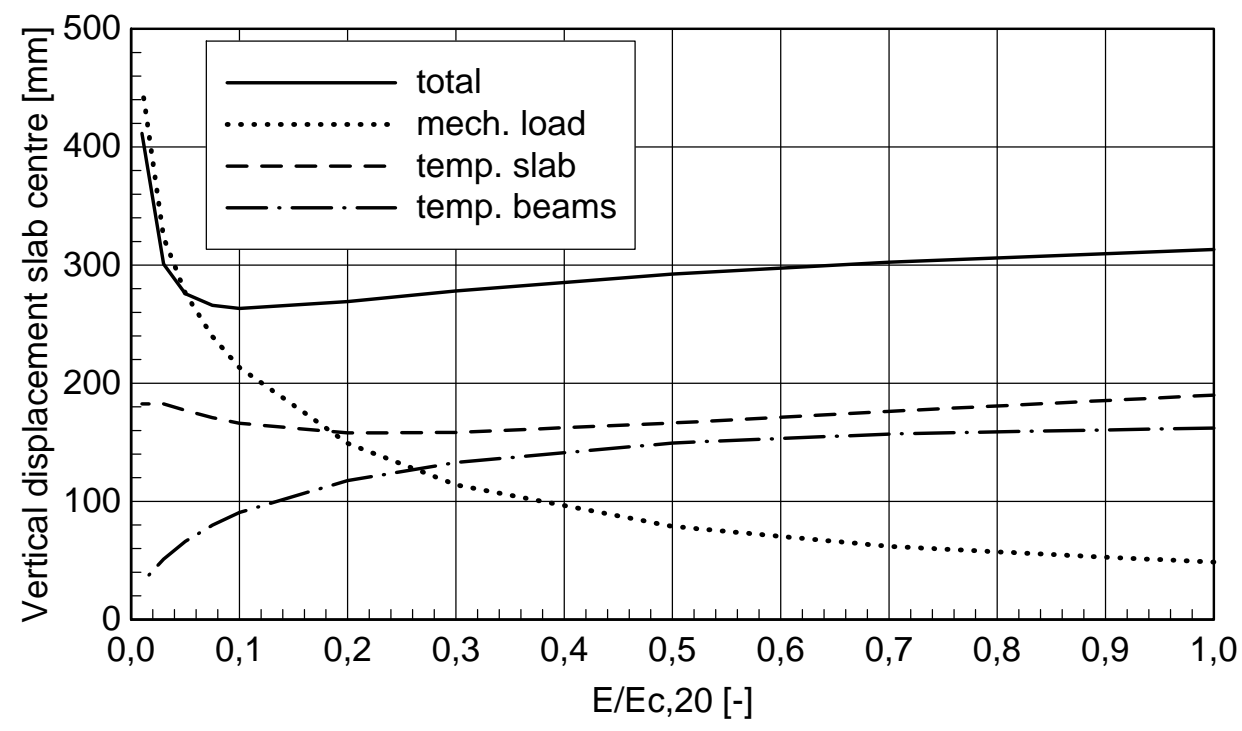

Fig. 5.5: Influence of the stiffness of the slab on displacement at slab centre

A parameter study is performed with this system in order to investigate the influence of the stiffness of the slab on the deformations and internal forces. The study starts with a Young's modulus of a concrete class $\mathrm{C} 20 / 25$ under $20^{\circ} \mathrm{C}$ of $E_{c, 20}=11884 \mathrm{~N} / \mathrm{mm}^{2}$ as determined in Table 4.4. This value is set to $100 \%$. It is reduced in several steps up to $1 \%$ of the initial stiffness. Lower stiffness leads to a instable system. The vertical deformation $w$ at the centre of the slab is shown in Figure 5.5. The total deformation is shown as well as the deformation caused by separate parts of the loading. The sum of the separate parts does not deliver the total deformation. These cannot be superposed since every load case has been solved as geometrically non-linear. It can be seen that the total deformation is relatively constant in a range between $5 \%$ and $100 \%$ of the initial stiffness. It is noticeable that the total deformations are 
smaller at lower than at higher stiffness. At a very low stiffness the deformations increase exponentially. With a high stiffness the deformation is mainly caused by the thermal elongation of the slab and beams whereas with a low stiffness the mechanical load governs the deformation. There the thermal elongation of the slab and beams even reduce the total displacement. This can be seen on the deformation only caused by the mechanical load which is higher than the total deformation.

The resulting bending moments $m_{x x}$ and $m_{y y}$ and membrane forces $n_{x x}$ and $n_{y y}$ in longitudinal and transversal direction at the slab centre are shown in Figure 5.6 and 5.7. It can be seen that all forces decrease considerably if the stiffness becomes lower. This is mainly due to the fact that a softer slab provides lower restraint to the thermal elongation and therefore, the restraint forces are lower with lower stiffness.

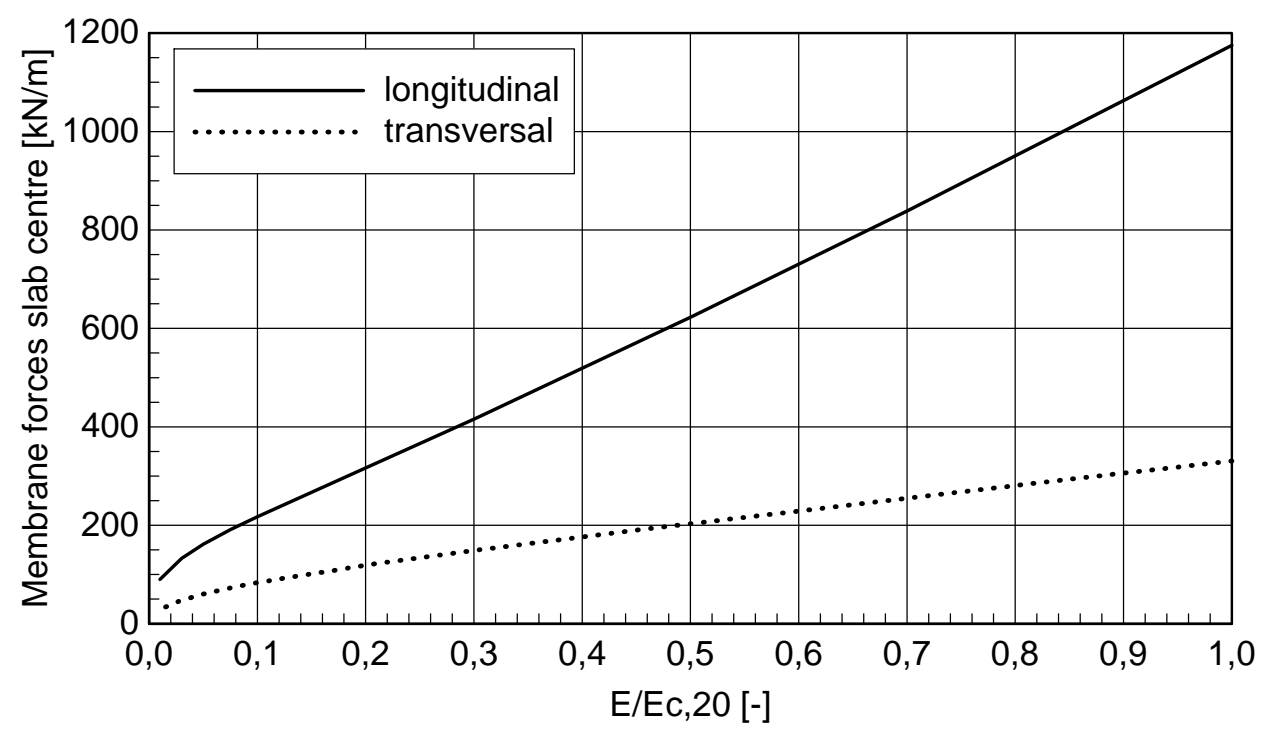

Fig. 5.6: Influence of the stiffness of the slab on membrane forces at centre of slab

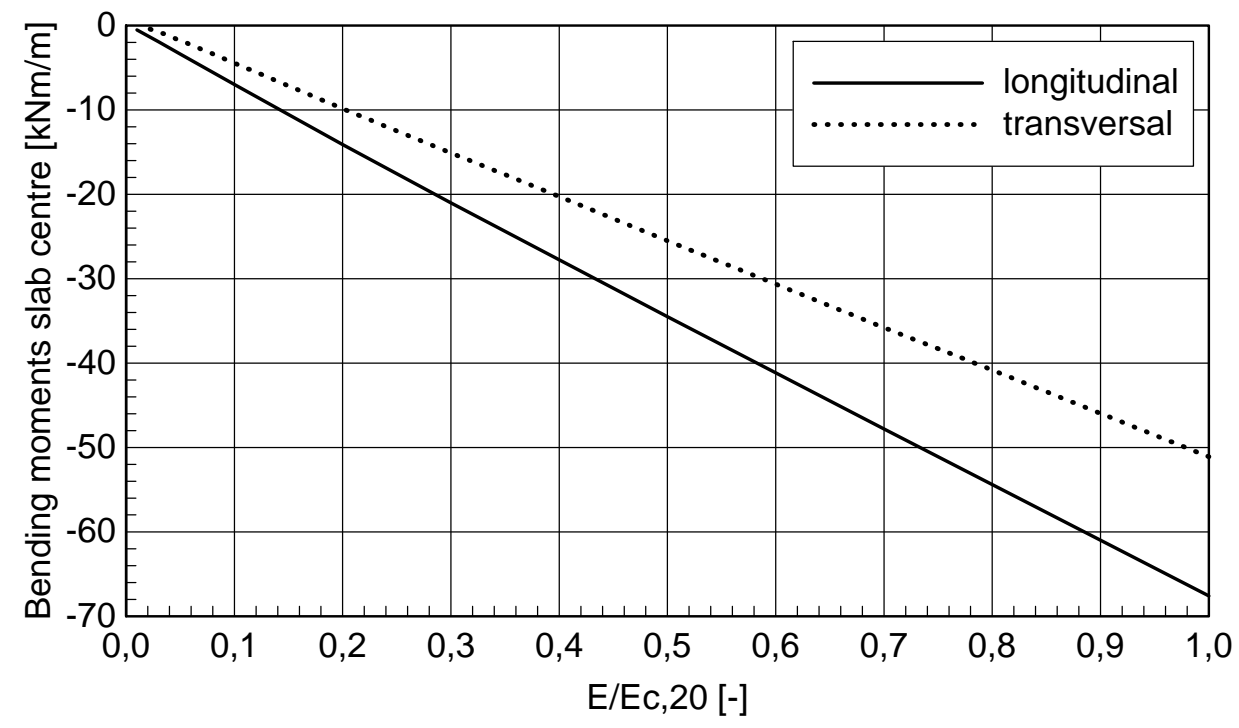

Fig. 5.7: Influence of the stiffness of the slab on bending moments at centre of slab 
It is noticeable that the bending moments have negative values which means that they are hogging moments and induce tension at the top surface of the slab. This can be explained with the following example which was published by the author in [43]. A beam as shown in Figure 5.8 is considered. It is heated non-uniformly with a higher temperature at the bottom than at the top surface. In a first step the beam is simply supported. Due to the thermal curvature the beam bows and the ends move together. In a statically determinate system this deformation causes no internal forces. If in a second step the horizontal deformations are restrained, like in the case of two-dimensional slab systems, the temperature change causes restraint forces. These forces are tensile normal forces and hogging moments. This can be illustrated by considering that the support of the beam in Figure 5.8 above is shifted to its initial position after the beam is heated.

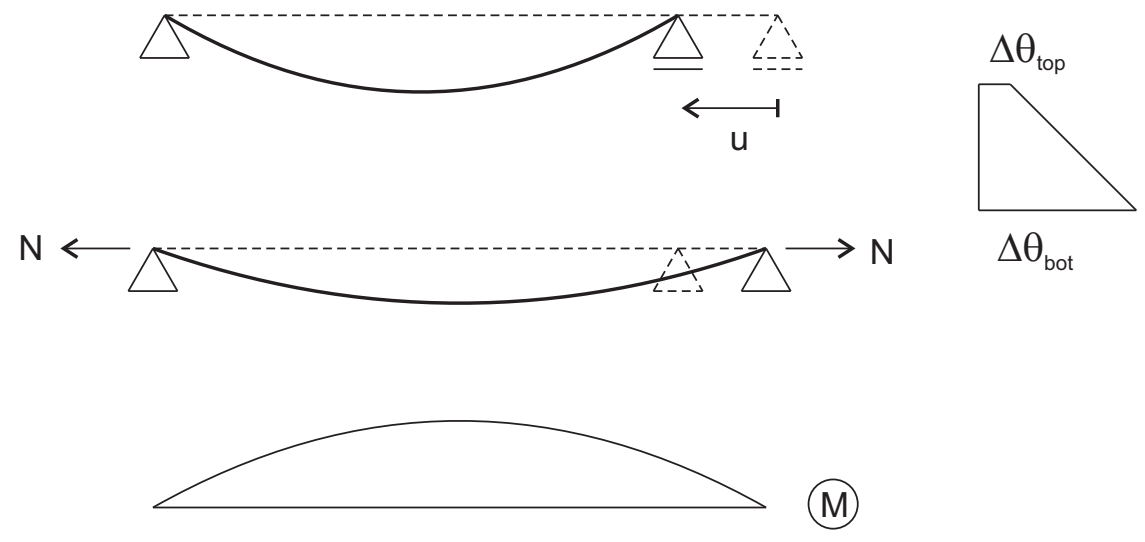

Fig. 5.8: Internal forces in restraint beam with non-uniform heating

The assumption, which is used in most simple calculation models, that the slab acts like an ideal membrane without bending stiffness and therefore the membrane forces are lower, the larger the deformations become, is only valid for a very small stiffness. At a stiffness range of about $5 \%$ to $100 \%$ the membrane forces are lower at a small stiffness although the deformations are higher at a large stiffness. Nevertheless the internal forces and, hence, the required reinforcement amount are always lower, the lower the stiffness of the slab is. The problem here is the limit of the lowest stiffness that is possible at the ultimate limit state. This problem is solved in the following sections.

\subsubsection{Tension stiffening}

Under ambient temperature the stiffness decrease of a reinforced concrete member under tension is caused by cracking of the concrete, however, the concrete cannot crack completely. Some parts of the concrete remain uncracked and increase the stiffness of the slab. This issue is usually called tension stiffening. A detailed description can be found in [55] for example. Here a brief summary is given. A reinforced concrete bar will be considered as shown in Figure 5.9. The bar is tensioned due to restraint forces, which occur for example in a horizontally restrained slab with temperature change. As long as the bar is uncracked, the strain of the concrete and the reinforcing bar (rebar) are similar everywhere. Once the tensile strength $f_{c t}$ and the relating cracking strain $\varepsilon_{c r}$ is reached at any location, the first crack occurs. The whole load has to be taken by the rebar which leads to a strain peak in the rebar in the crack. The strain of the concrete is zero in the crack. Further tensile forces are transferred from the rebar in the crack to the uncracked concrete by the bond between them. As soon as the bond reaches the tensile strength of the concrete, the next crack occurs. The bond needs a certain length to fully develop. This 
length $l_{t}$ is called transfer length. In addition to the tensile strength of the concrete, the transfer length governs the crack spacing. It depends on the one hand on the quality of the bond. Ribbed rebar provide a better bond than plain rebar. On the other hand it depends on the available surface of the rebar. A larger amount of reinforcement with smaller diameters provide a larger surface than a smaller amount with larger diameters. The cracking of the bar is finished when in no further uncracked part the bond can reach the tensile strength of the concrete.

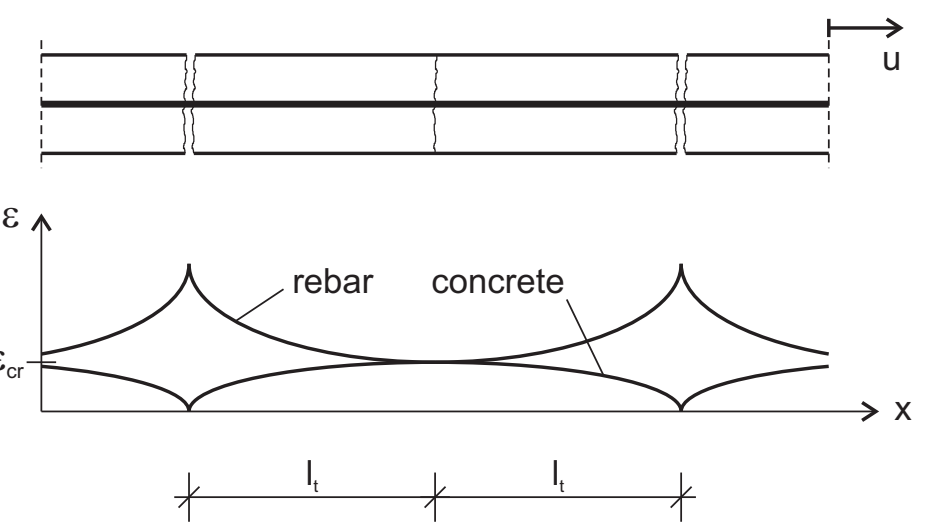

Fig. 5.9: Crack development on the basis of [55]

The cracking behaviour decisively depends on the available reinforcement in the slab. Beside the bond strength as described above, it is mainly influenced by the reinforcement ratio. This can be seen in Figure 5.10. A reinforced concrete bar is again tensioned due to restraint forces. The elongation causes an induced normal force $N_{\text {ind }}$. The diagrams show this force as a function of the total elongation $u$. The dashed lines show how a pure reinforcement bar would behave if no concrete was available. At the upper part of Figure 5.10 the bar has a high reinforcement ratio. The first crack occurs when the tensile strength of the concrete $f_{c t}$ is reached. The bar is further tensioned and all cracks develop as described above. The end of the cracking is marked in the diagram in Figure 5.10 above by the end of the serrated
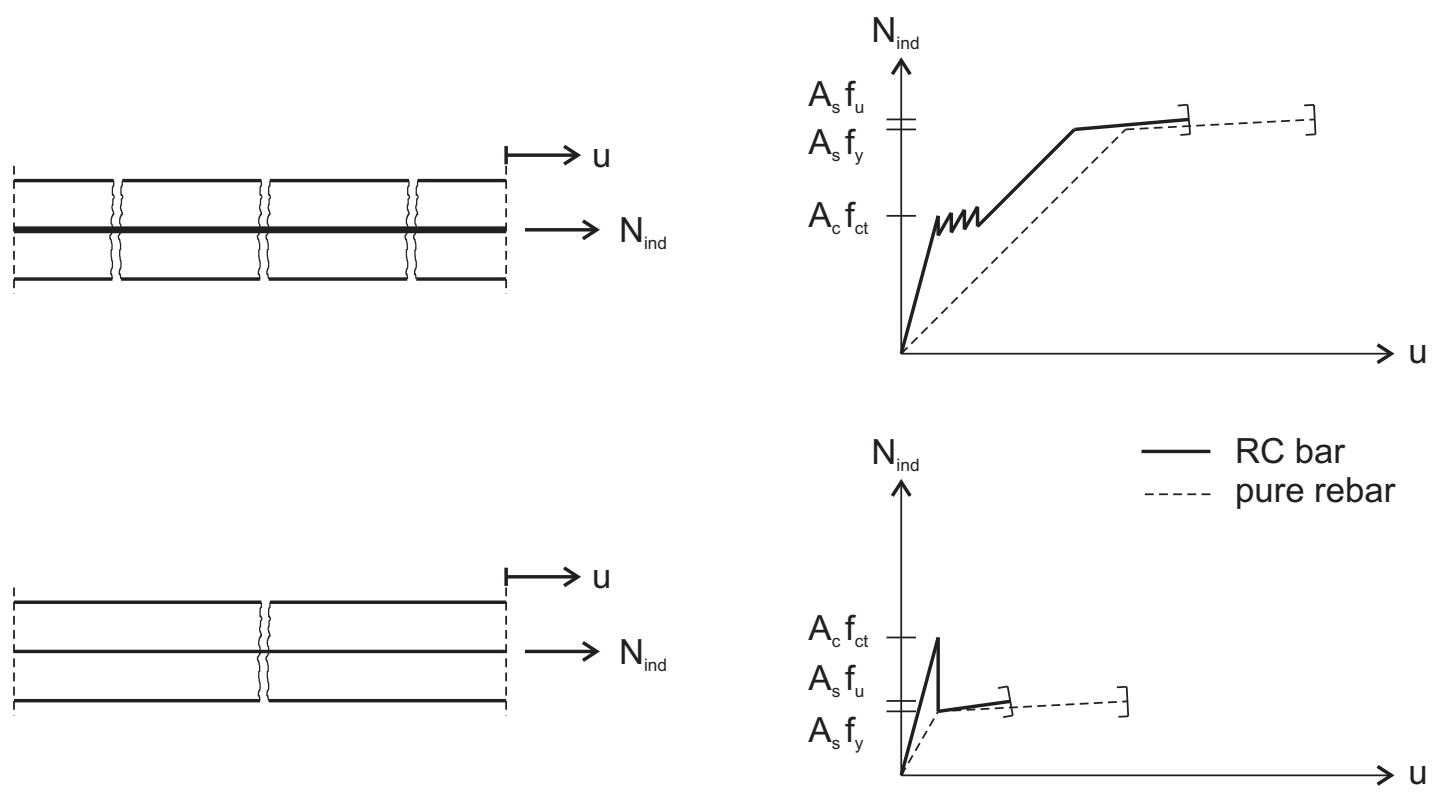

Fig. 5.10: Simplified cracking behaviour of a reinforced concrete bar with high (above) and low (below) reinforcement ratio on the basis of [55] 
line. Now every part of the bar is tensioned further including the rebar in the cracks and the uncracked parts. The stress-strain curve runs parallel to the line of the pure rebar until the yield strength $f_{y}$ of the reinforcement is reached. The total deformation of the whole bar is smaller, it behaves stiffer. This is the origin of the expression tension stiffening. After reaching the yield strength, the reinforcement starts to yield in the cracks until it ruptures at its ultimate strength $f_{u}$. This rupture occurs at a much lower total deformation than at a pure rebar since the strains concentrate in the cracks and the ultimate strain in the cracks is reached sooner.

The lower part of Figure 5.10 shows a slightly reinforced concrete bar. There the first crack also occurs when the tensile strength of the concrete is reached. Again, in the crack the whole force has to be taken by the reinforcement, however, the bearable force of the reinforcement $N_{s}=A_{s} f_{y}$ is smaller than the force that is required to induce the first crack $N_{c r}=A_{c} f_{c t}$. Therefore, the induced force $N_{\text {ind }}$ drops to $N_{s}$. The reinforcement in the crack is not able to transfer enough force into the concrete in order to induce a second crack since $N_{s}$ is smaller than $N_{c r}$. If the bar is tensioned further, the reinforcement in the crack yields and finally ruptures at its ultimate strength $f_{u}$. If the force, that is required to induce the first crack $N_{c r}$, is even higher than the ultimate strength of the rebar $A_{s} f_{u}$, the reinforcement may rupture as soon as the first crack occurs. This can happen not only if the deformation is induced by an external load but also by deformations due to restraint. This kind of brittle failure is usually supposed to be avoided since it happens suddenly and unheralded. All design methods for calculating minimum reinforcement amounts to avoid brittle failure in the Eurocode and other design codes are therefore based on the described assumptions. The same is true for design methods for crack control reinforcement. All these methods require to cover at least the tensile strength of the concrete with reinforcement in order to enable that several cracks can arise and prevent the first crack from gaping.

\subsubsection{Stiffness at the ultimate limit state}

For concrete and composite slabs in case of fire with partly unprotected secondary beams it can be assumed that the stiffness of the slab is governed by the tensile stiffness. The bending stiffness is very small since the depth of the slabs is very small compared to the spans, and the concrete cracks. Furthermore, the large deformations generate a membrane system whose tensile stiffness is much higher than the bending stiffness. In addition, almost the whole slab is under tension due to tensile membrane action at the slab centre and restrained thermal elongation of the edge beams and unprotected secondary beams.
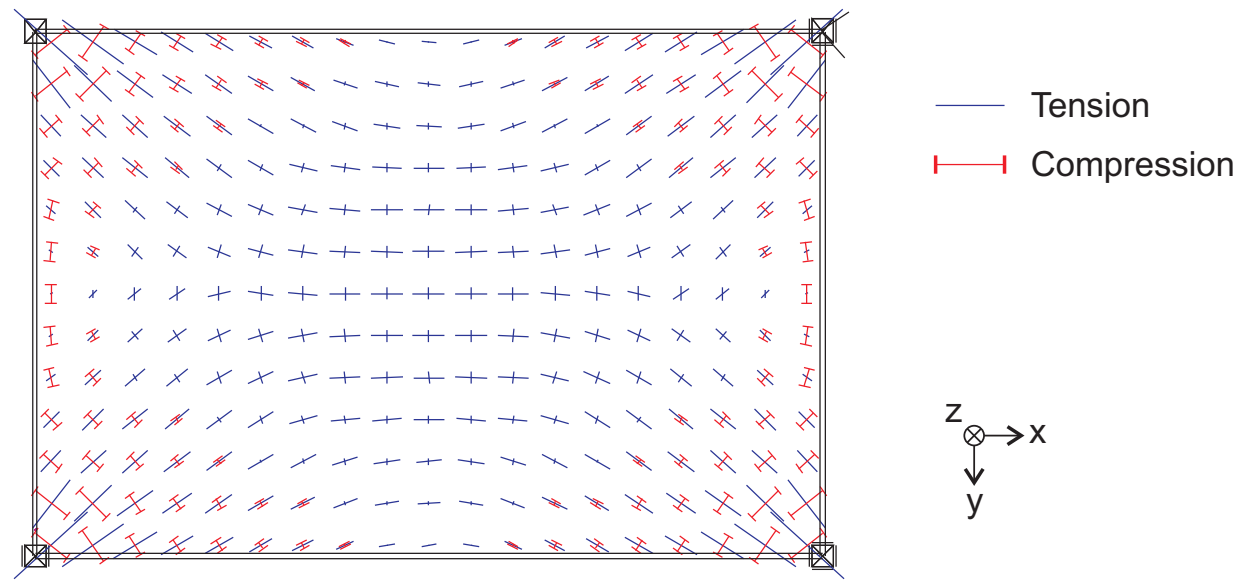

Fig. 5.11: Membrane forces of a single slab panel 
This can be seen in Figure 5.11. It shows a slab of Example 5-1 with a resulting stiffness of $10 \%$ of the stiffness at ambient temperature. Apart from some comressive zones across the corners the whole slab is in tension.

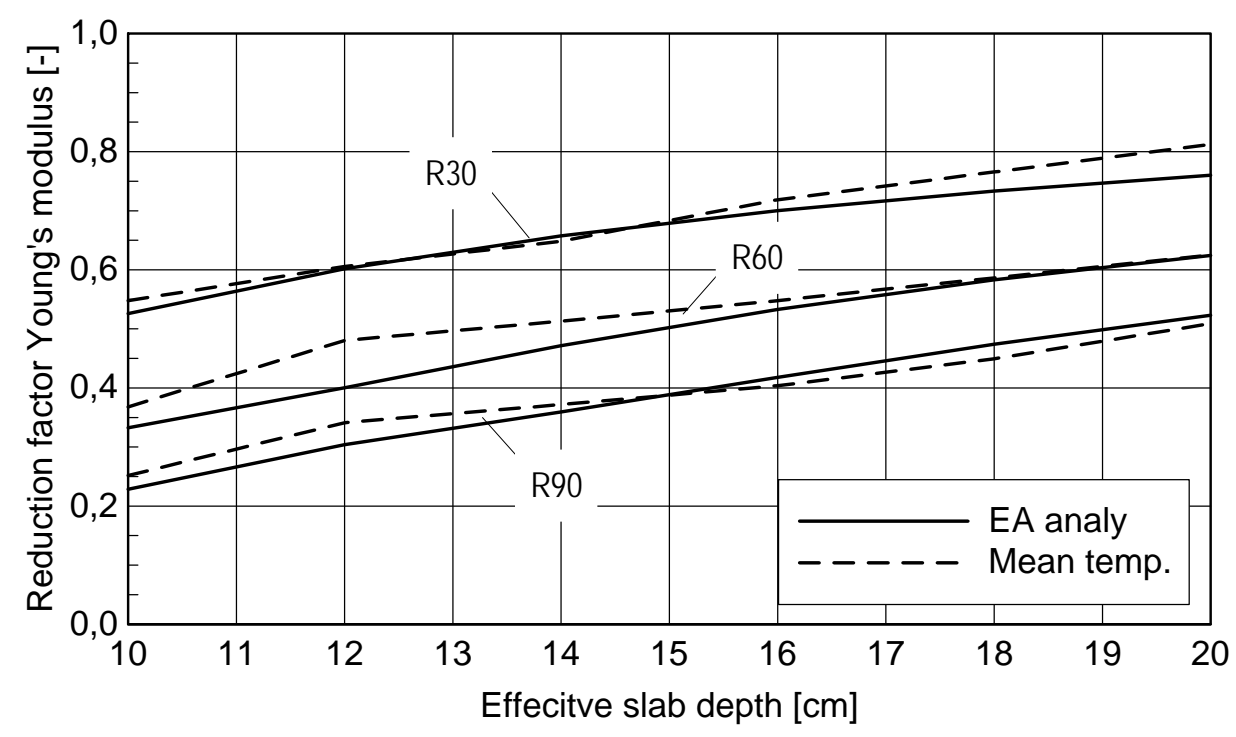

Fig. 5.12: Reduction of stiffness due to softening of concrete

In case of fire the stiffness reduction of a slab is caused by two effects: softening of the concrete due to heating and cracking of the concrete. The softening is quantified for three times of the standard fire R30 to R90 as shown in Figure 5.12. The values of the solid lines are calculated by the resulting tensile stiffness EA of heated full concrete slabs with Equation (4.18) as described in Section 4.2.2 divided by the stiffness at $20^{\circ} \mathrm{C}$. These values show the reduction rate of the uncracked stiffness of a heated cross-section compared to its stiffness under ambient temperature. It can be seen that the higher the temperatures in the cross-section are, the more the stiffness is reduced. Thinner and longer heated slabs become hotter than thicker and shorter heated slabs. The values of the dashed lines are the reduction factors of the Young's modulus $k_{E, \theta}$, derived in Section 4.2.1 Table 4.5, for a concrete temperature at the centre of the cross-section. These temperatures are taken from DIN EN 1994-1-2, Table D.5 [32] where temperature distributions of a $100 \mathrm{~mm}$ thick slab are given for several times of the standard fire curve. It can be seen that the curves show good comparison.

Table 5.1: Reduction factor $k_{E, \theta, \text { mean }}$ for cross-section softening

\begin{tabular}{|c|c|c|c|}
\hline & $\mathrm{R} 30$ & $\mathrm{R} 60$ & $\mathrm{R} 90$ \\
\hline$h$ or $h_{\text {eff }}$ & \multicolumn{3}{|c|}{$k_{E, \theta, \text { mean }}$} \\
\hline$[\mathrm{cm}]$ & \multicolumn{3}{|c|}{$[-]$} \\
\hline 10 & 0.548 & 0.368 & 0.252 \\
\hline 12 & 0.606 & 0.480 & 0.341 \\
\hline 14 & 0.648 & 0.513 & 0.372 \\
\hline 16 & 0.719 & 0.548 & 0.404 \\
\hline 18 & 0.766 & 0.586 & 0.449 \\
\hline 20 & 0.813 & 0.625 & 0.509 \\
\hline
\end{tabular}

The effect of concrete softening on the stiffness reduction can be taken into account simply by a 
reduction factor $k_{E, \theta, \text { mean }}$. No explicit thermal analysis is required to determine this factor since the temperatures of the table in the Eurocode can be used. For composite slabs the slab depth $h$ has to be replaced by the effective depth $h_{\text {eff }}$ calculated according to DIN EN 1994-1-2, Annex D [32]. Some values of $k_{E, \theta \text {,mean }}$ are prepared in Table 5.1. Intermediate values can be interpolated.

Tension stiffening effects are taken into account in the presented method with the following assumptions:

- The whole slab is under tension as described above.

- Only the top reinforcement is considered for the determination of the resulting stiffness. The bottom reinforcement in full concrete slabs becomes very soft since it reaches a very high temperature. The contribution on the stiffness can therefore be neglected.

- The steel sheeting of composite slabs also becomes very soft due to high temperatures and it possibly debonds from the concrete. Its contribution on the stiffness can also be neglected.

- The concrete around the top reinforcement remains very cool. Therefore, tension stiffening can be taken into account with assumptions for ambient temperature.

- The amount of available reinforcement is enough that it does not yield due to the determined internal forces.

- The amount of available reinforcement is enough that the cracking force caused by the tensile strength of the concrete can be transferred and a distributed crack pattern can develop as described in Section 5.2.3.

- For determining the resulting stiffness with tension stiffening it is assumed that the reinforcement just reaches the yield strength.

With these assumptions available tension stiffening approaches for ambient temperature can be used. The following approaches are based on the book of Zilch and Zehetmaier [55], Section 10.3.3 and the DIN-Fachbericht 102 [34], Annex 2 which is a collection of codes and regulations for the design of concrete bridges in Germany on the base of the Eurocodes. In Figure 5.13 the stress-strain curves and the meaning of the used symbols are shown. The dash-dot line shows the stress-strain curve of a pure reinforcement bar. The thin solid line is a plot of the stresses of a reinforcement bar embedded in concrete as a function of its strain. The thick solid line shows the resulting stiffness of the reinforced concrete bar relating to the reinforcement area.

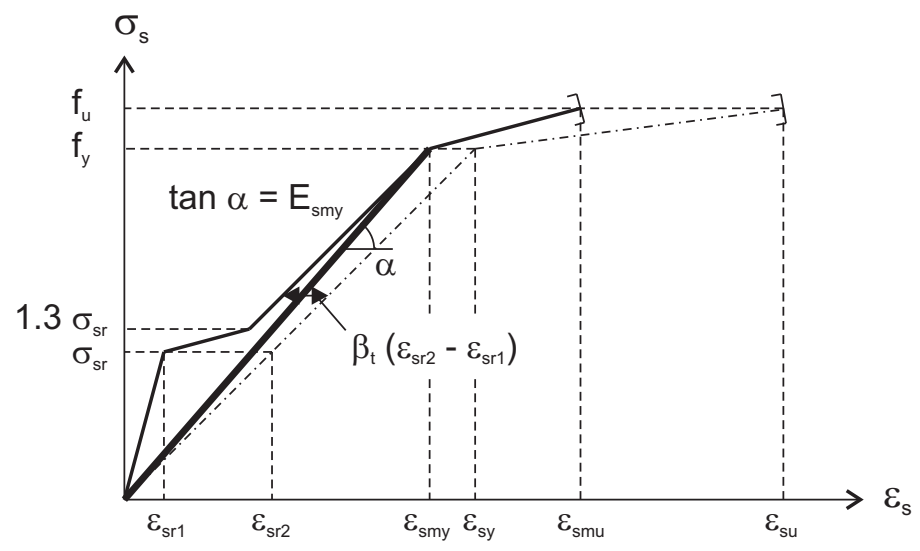

Fig. 5.13: Tension stiffening on the base of [55]

The symbols in Figure 5.13 and in the following equations mean:

$\varepsilon_{s r 1} \quad$ Steel strain in uncracked bar under cracking normal force when $f_{c t, \text { eff }}$ is reached. 
$\varepsilon_{s r 2} \quad$ Steel strain in crack under cracking normal force.

$\varepsilon_{s m y} \quad$ Mean value of steel strain under yielding normal force when $f_{y}$ is reached.

$\varepsilon_{s y} \quad$ Steel strain in cracks under yielding normal force.

$\varepsilon_{s m u} \quad$ Mean value of steel strain under ultimate normal force when $f_{u}$ is reached.

$\varepsilon_{s u} \quad$ Steel strain in cracks under ultimate normal force.

$\sigma_{s r} \quad$ Stress in reinforcement bar in the first crack under cracking normal force.

$f_{y} \quad$ Yield strength of reinforcement bar.

$f_{u} \quad$ Ultimate strength of reinforcement bar.

$f_{c t, e f f} \quad$ Effective tensile strength of the concrete.

$=f_{c t m}$ according to DIN EN 1992-1-1, Table 3.1 [26] but $f_{c t, e f f} \geq 3.0 \mathrm{~N} / \mathrm{mm}^{2}$

$\beta_{t} \quad=0.25$. Coefficient taking into account the duration of loading.

$E_{S} \quad$ Young's modulus of the reinforcement steel.

$E_{s m y} \quad$ Mean value of Young's modulus of steel under yielding normal force.

$E_{c, e f f} \quad$ Effective Young's modulus of concrete.

$=E_{c m}$ according to DIN EN 1992-1-1, Table 3.1 [26] but $E_{c, e f f} \geq 33000 \mathrm{~N} / \mathrm{mm}^{2}$

$E_{c m}^{I I} \quad$ Mean value of Young's modulus of cracked concrete.

$\rho_{c} \quad$ Reinforcement ratio relating to the whole concrete area $A_{c}$

$\rho_{c, e f f} \quad$ Reinforcement ratio relating to the effective tension area $A_{c t, e f f}$.

$h \quad$ Total slab depth

$h_{\text {eff }} \quad$ Effective slab depth, for full concrete slabs $h_{\text {eff }}=h$,

for composite slabs see DIN EN 1994-1-2, Annex D [32].

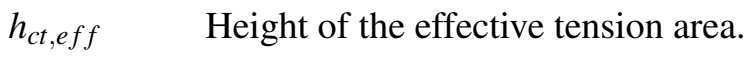

$=2.5 d_{1} \geq h_{\text {eff }} / 2 \leq h_{\text {eff }}$

The tensile strength of concrete varies statistically in a broad range. Therefore, usually a mean value $f_{c t m}$ is used, for example in Eurocode 2 for determining a minimum reinforcement area for brittle failure. In the German national annex of DIN EN 1992-1-1 [28] it is specified that the effective tensile strength $f_{c t, e f f}$ should be at least $3.0 \mathrm{~N} / \mathrm{mm}^{2}$. This value corresponds roughly to a concrete class C30/37 and takes into account that particularly lower concrete classes often have a higher strength in reality than required. This argumentation is adopted in this work. The same is true for the effective Young's modulus of concrete $E_{c, e f f}$. For this a minimum value should also be used. In this work the value of a concrete class $\mathrm{C} 30 / 37$ is proposed. The coefficient $\beta_{t}$, which takes into account the duration of the loading, is set to 0.25 in this work since no values for the case of fire are available. The values explained above can be determined as follows:

$$
\begin{aligned}
& \varepsilon_{s r 1}=\frac{f_{c t, e f f}}{E_{c, e f f}} \\
& \sigma_{s r}=f_{c t, e f f}\left(\frac{1}{\rho_{c, e f f}}+\frac{E_{s}}{E_{c, e f f}}-1\right) \\
& \varepsilon_{s r 2}=\frac{\sigma_{s r}}{E_{s}} \\
& \varepsilon_{s m y}=\varepsilon_{s y}-\beta_{t}\left(\varepsilon_{s r 2}-\varepsilon_{s r 1}\right) \\
& \varepsilon_{s y}=\frac{f_{y}}{E_{s}}
\end{aligned}
$$




$$
\begin{aligned}
& E_{s m y}=\frac{f_{y}}{\varepsilon_{s m y}} \\
& (E A)_{s m}=E_{s m y} A_{s} \\
& E_{c m}^{I I}=\frac{(E A)_{s m}}{A_{c}}
\end{aligned}
$$

With Equations (5.10) to (5.17) the resulting Young's modulus of slabs in case of fire $E_{c m, \theta}^{I I}$ can be determined. In Equation (5.18) the reduction factor for cross-section softening $k_{E, \theta, m e a n}$ of Table 5.1 is included.

$$
E_{c m, \theta}^{I I}=k_{E, \theta, \text { mean }} E_{c m}^{I I}=k_{E, \theta, \text { mean }} \frac{f_{y} \rho_{c}}{\frac{f_{y}}{E_{s}}-\beta_{t}\left[\frac{f_{c t, e f f}}{E_{s}}\left(\frac{1}{\rho_{c, e f f}}+\frac{E_{s}}{E_{c, e f f}}-1\right)-\frac{f_{c t, e f f}}{E_{c, e f f}}\right]}
$$

Where:

$$
\begin{aligned}
& \rho_{c}=\frac{A_{s}}{A_{c}}=\frac{A_{s}}{b \cdot h_{e f f}} \\
& \rho_{c, e f f}=\frac{A_{s}}{A_{c t, e f f}}=\frac{A_{s}}{b \cdot h_{c t, e f f}}
\end{aligned}
$$

The effective tension area $A_{c t, e f f}$ is assumed on the base of Eurocode 2 [26] as shown in Figure 5.14. The origin is explained in detail in [55]. Generally the height of the tension area is 2.5 times the distance of the reinforcement from the tensioned surface $d_{1}$. This value has been adopted in the Eurocode since the experts agree about it. For membrane action it needs to be enhanced. For example if the upper reinforcement is placed very close to the top surface of the slab, the effective tension area would be very small. The larger the effective tension area is, however, the higher are the resulting stiffness of the slab and the resulting internal forces. The worst case is therefore a tension area as large as possible. This area should at least cover half of the slab depth at full concrete slabs or half of the effective depth at composite slabs $h_{c t, e f f} \geq h_{e f f} / 2$, but it cannot be larger than the slab depth or effective depth $h_{c t, e f f} \leq h_{\text {eff }}$.
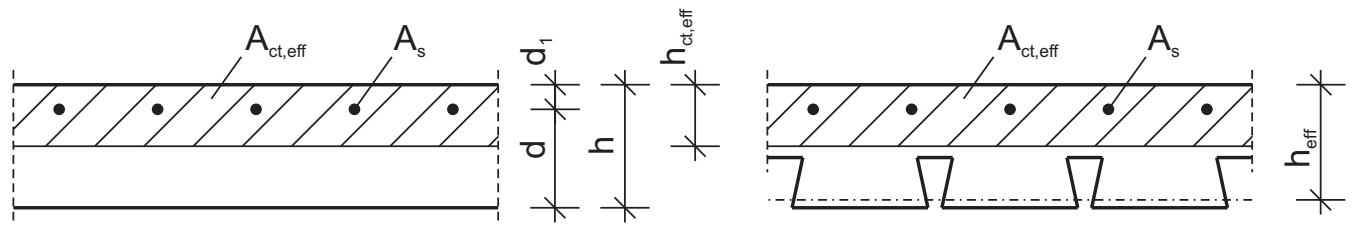

Fig. 5.14: Effective tension area on the base of [26]

The assumptions above for determining the resulting Young's modulus are only valid if the amount of available reinforcement is great enough, that the cracking force caused by the tensile strength of the concrete can be transferred and a distributed crack pattern can develop as described in Section 5.2.3. In order to ensure this, a minimum reinforcement area $A_{s, \min }$ is required. An equation for $A_{s, \min }$ can be determined as follows using Equation (5.11) and the fact that the available reinforcement has to be able to bear at least the cracking force. The steel stress in the crack is limited to the yield strength $f_{y}$.

$$
\begin{aligned}
& \sigma_{s r} \leq f_{y} \\
& f_{c t, e f f}\left(\frac{1}{\rho_{c, e f f}}+\frac{E_{s}}{E_{c, e f f}}-1\right) \leq f_{y}
\end{aligned}
$$




$$
\begin{aligned}
\rho_{c, e f f} & \geq \frac{1}{\frac{f_{y}}{f_{c t, e f f}}-\frac{E_{s}}{E_{c, e f f}}+1} \\
\rho_{c, e f f} & =\frac{A_{s}}{A_{c t, e f f}} \\
A_{s, \text { min }} & =\frac{A_{c t, e f f}}{\frac{f_{y}}{f_{c t, e f f}}-\frac{E_{s}}{E_{c, e f f}}+1}
\end{aligned}
$$

The orthotropy of composite slabs can be neglected during the determination of the resulting stiffness. The different cross-sections in longitudinal and transversal direction mainly influence the bending stiffness. The bending stiffness only plays a minor role at membrane action as described above. The resulting stiffness is governed by the tensile stiffness which is determined on the effective tension area around the upper reinforcement. The largest part of this area is above the troughs of the steel sheeting and is therefore hardly influenced by the troughs. For these reasons a similar stiffness in both directions can be assumed for composite slabs. A different stiffness only has to be taken into account if a different amount of reinforcement is used in longitudinal and transversal directions. This is true for both full concrete and composite slabs.

With Equation (5.18) a very simple method is provided to calculate the minimum possible stiffness at the ultimate limit state. A minimum reinforcement area has to be used which can be determined with Equation (5.25). During the design of concrete and composite slab systems in practice, a calculation should firstly be performed with a stiffness of the slab determined by the minimum reinforcement area. If the required amount of reinforcement for the internal forces is larger than the minimum reinforcement, a second calculation with higher stiffness needs to be performed.

\subsection{Modelling of the beams}

Different options are possible to model the composite beams. The simplest option is to replace the steel beams by rigid supports. This is assumed in most simple calculation models. However, important aspects are neglected with this assumption as discussed later on. At ambient temperature whole composite beams are often modelled with one beam element which includes the whole cross-section with the steel beam and concrete flange. The biggest advantage of this option is that the resulting internal forces in the composite beam can be determined directly. These are not split into parts like if the steel beam and concrete flange are modelled separately. With these resulting forces the cross-section can be designed with simple calculation methods. In the case of fire however the temperature distribution is difficult to take into account. For every combination of steel section, slab system and slab depth a separate substitute thermal loading would be necessary. Furthermore, large deformations and membrane forces perpendicular to the beam span cannot be taken into account. It is therefore useful to model the steel section and the slab separately. The design of the composite beam is more difficult in this case but the modelling is much easier and the behaviour of the whole beam slab system is considered more exactly. This approach is used in Vulcan and SlabFem and also in this work.

The steel beams can be modelled with two types of finite elements: shell elements or beam elements. With shell elements different temperatures in the single flanges and the web can be taken into account. These differences can be neglected as described in Section 4.3. In order to model an I-beam with shell elements, at least five elements are necessary over the cross-section. This can be seen in Figure 5.15 a). 
The effort is therefore relatively high with these elements. Since a uniform temperature distribution in the steel section is exact enough, beam elements are the most useful option.

A further issue is the connection of the beam elements and the slab. In Figure 5.15 three possibilities are shown. The most exact option is b) which is the same as a). Two additional nodes are input at the intersection of the beam and the slab. Each of the two nodes are rigidly coupled with the slab above or the below beam. At the two additional nodes only the displacements are coupled, no rotations. The coupling of the two additional nodes in the direction of the beam axis can be replaced by springs in order to take into account the stiffness of shear studs or slip in the shear connection. In this work the shear studs and connection are assumed to be rigid since it delivers reasonable results compared to test data and no research results are available on this topic for the fire case. Four lines of nodes have to be modelled at every beam with this option. In Figure $5.15 \mathrm{c}$ ) the beam elements are rigidly coupled directly with the slab. With this option only two lines of nodes have to be modelled. The option with the smallest modelling effort is shown in Figure $5.15 \mathrm{~d}$ ). There the same nodes are used for the slab and the beam. This option is only possible if the finite element software includes beam elements which can be connected eccentrically to the nodes.
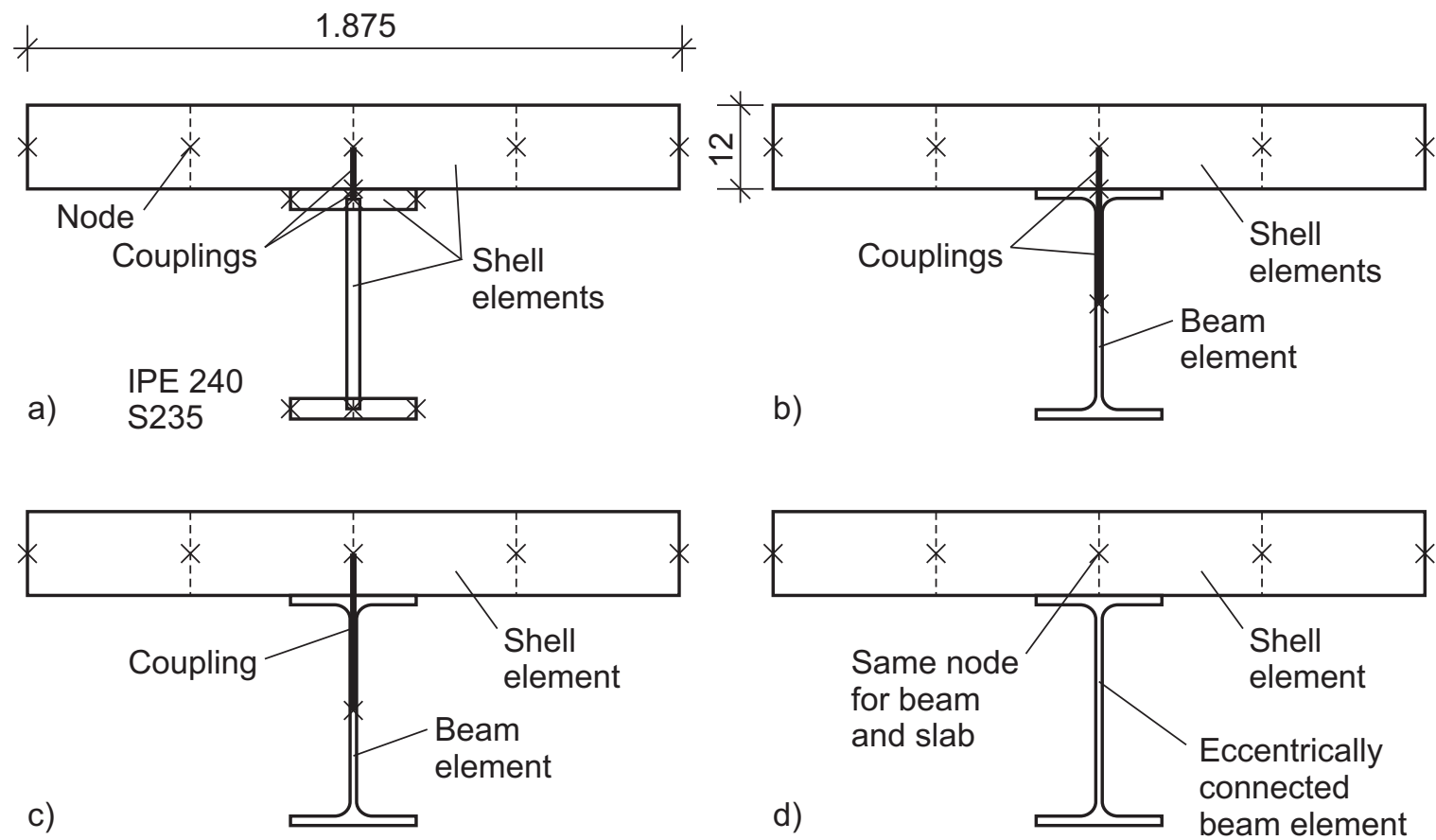

Fig. 5.15: Example 5-2: Options for beam modelling

For concrete and composite slab systems in case of fire all three options can be used which can be proved as follows. A composite beam is considered in Example 5-2 with the same system and crosssection as Example 4-3. The beam is simply supported with a length of $7.5 \mathrm{~m}$ and exposed to the standard fire for thirty minutes from below. The system can be seen in Figure 4.20 and the cross-section in Figure 5.15. The slab is loaded with a substitute thermal loading of $\varepsilon_{\theta, \text { subs }}=0.791 \%$ and $\kappa_{\theta, \text { subs }}=$ $32.65 \mathrm{~km}^{-1}$ from Table 4.6. The steel section is assumed to reach $808^{\circ} \mathrm{C}$ uniformly like in Example 4$3 \mathrm{c}$. The temperature is taken into account with a substitute thermal loading of $\varepsilon_{\theta \text {,subs }}=11.0 \%$ and a reduced Young's modulus of $E_{\theta}=18520 \mathrm{MPa}$, both according to DIN EN 1994-1-2 [32]. The resulting deflection $w$ at midspan and the elongation $u$ of Example 4-3c which corresponds to Example 5-2a and the three examples with beam elements 5-2b to d are compared in Table 5.2. The differences are negligible. 
This confirms that all of the modelling options can be used. Although d) is the simplest option, c) is used further in this work since plastic hinges in the steel beams can be included. With Sofistik option c) leads to numerically more stable systems. The input file of Example 5-2c can be found in Annex B.4.

Table 5.2: Comparison of results of Example 5-2

\begin{tabular}{|c|c|c|}
\hline Example & $w$ & $u$ \\
\hline & {$[\mathrm{mm}]$} & {$[\mathrm{mm}]$} \\
\hline $5-2 \mathrm{a}$ & 247.9 & 6.26 \\
\hline $5-2 \mathrm{~b}$ & 248.7 & 6.30 \\
\hline $5-2 \mathrm{c}$ & 249.1 & 6.37 \\
\hline $5-2 \mathrm{~d}$ & 249.1 & 6.38 \\
\hline
\end{tabular}

The material of the steal beams can be considered to be linear-elastic. Material non-linearities are taken into account with a reduced Young's modulus and plastic hinges. The Young's modulus is reduced due to the steel temperature according to DIN EN 1993-1-2 [29] or DIN EN 1994-1-2 [32]. Bending hinges should be modelled at the ends of every steel beam as shown in Figure 5.16. This assumption is possible if only the ultimate limit state is considered. Due to the large rotations of the beam, a large bending moment occurs at the supports, the cross-section yields and a bending hinge arises. As described in Section 5.1.2 the forces in the plastic hinges can be conservatively neglected and perfect hinges can be assumed. The resulting static system is a simply supported beam. The load bearing capacity of this beam is reached when the loads reach the plastic bending resistance of the hot composite cross-section at midspan. Catenary action due to large deformations is conservatively neglected.
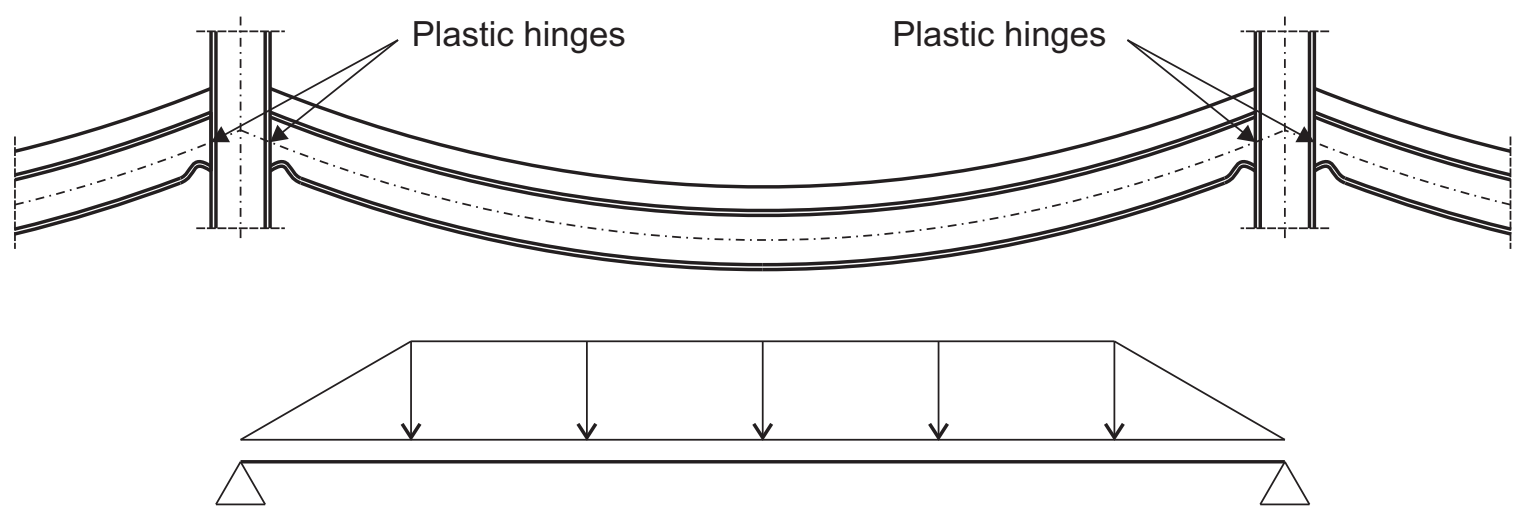

Fig. 5.16: Plastic hinges in steel beams and resulting static system of the edge beams

It is very important to model the steel beams since these highly influence the forces in the slabs above. This shall be discussed in the following example. The edge beams of the slab of Example 5-1 are replaced by rigid supports as assumed in the current available simple calculation models. The distribution of the membrane forces is shown in Figure 5.17. A classical compression ring and tensile forces in the slab centre can be found. If the edge beams are included in the model however, the force distribution changes dramatically as shown in Figure 5.11. No clear compression ring occurs in this model. In fact the tensile forces are in equilibrium with compressive forces in the edge beams. Depending on the thermal elongation and stiffness of the edge beams, there are either tensile forces in the concrete slab around the perimeter or maybe very small compressive forces. In Example 5-1 the edge beams in longitudinal direction are stiffer than in transversal direction since these have a larger cross-section area. Larger 
restraint forces will therefore occur in the longitudinal beams. These restraint forces are compressive forces in the beams which induce tension in the slab. The restraint forces in the transversal edge beams are not large enough to relieve the compression ring in the slab entirely and so small compressive forces still occur in the concrete. In addition, a part of the forces in the slab around the edges is the compressive component of the composite edge beams. These forces are overlaid with the compression ring and the restraint forces. The assumption that a pure compression ring forms in the concrete is only valid if the edge beams are neglected and replaced by rigid supports.

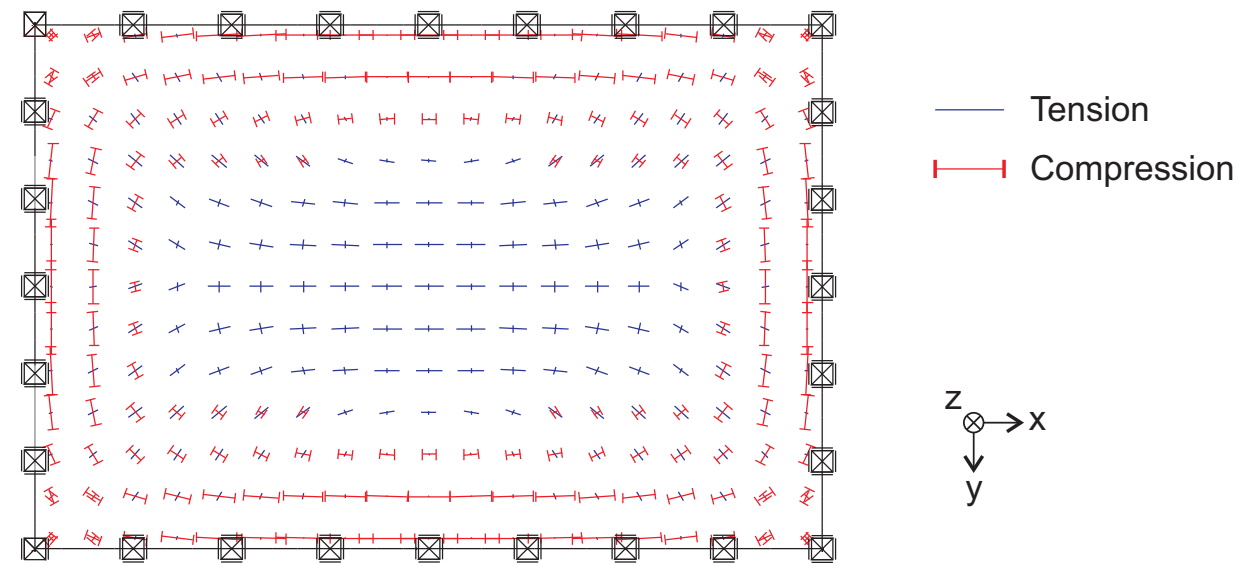

Fig. 5.17: Membrane forces of a single slab panel with rigid supports

In order to quantify the impact of the steel beams, the slab of Example 5-1 is modelled with different beam arrangements. At the centre of the slab, as shown in Figure 5.4, the vertical deformation $w$, the bending moments $m_{x}$ in longitudinal direction and $m_{y}$ in transversal direction as well as the membrane forces $n_{x}$ and $n_{y}$ in both directions are calculated. One slab is modelled with rigid supports, the other three include edge beams (EB). The temperature of the edge beams in the second slab is assumed with $20^{\circ} \mathrm{C}$. These beams are relatively stiff and no thermal elongation occurs. In the third slab the edge beams reach $540^{\circ} \mathrm{C}$. In the fourth slab two unprotected secondary beams (UB) IPE 200 in transversal direction are also modelled with a temperature of $1000^{\circ} \mathrm{C}$. The results are compared in Table 5.3.

Table 5.3: Comparison of results of a single slab panel with different support conditions

\begin{tabular}{|l|c|c|c|c|c|}
\hline & $w$ & $m_{x}$ & $m_{y}$ & $n_{x}$ & $n_{y}$ \\
\hline & {$[\mathrm{mm}]$} & {$[\mathrm{kNm} / \mathrm{m}]$} & {$[\mathrm{kNm} / \mathrm{m}]$} & {$[\mathrm{kN} / \mathrm{m}]$} & {$[\mathrm{kN} / \mathrm{m}]$} \\
\hline rigid supports & 280 & -6.39 & -0.90 & 205 & 37.6 \\
\hline EB 20 ${ }^{\circ} \mathrm{C}$ & 284 & -6.38 & -0.21 & 124 & 46.7 \\
\hline EB 540 ${ }^{\circ} \mathrm{C}$ & 263 & -6.99 & -4.45 & 217 & 83.7 \\
\hline EB $+\mathrm{UB}$ & 263 & -7.34 & -4.07 & 217 & 102 \\
\hline
\end{tabular}

It can be seen that the deformations do not differ very much. With heated beams these are slightly smaller since the thermal elongation in the beams stretches the slab. This can be compared with a stretched canvas which sags less the higher the frame is prestressed. The bending moments are larger if heated beams are taken into account, particularly in transversal direction $m_{y}$. This also can be explained by thermal elongation. As described in Section 5.2.2 the hogging moments develop due to horizontal restraint. If heated beams stretch the slab, the horizontal restraint of the slab becomes stiffer. The largest differences can be seen in the membrane forces which are much higher in the systems with heated steel 
beams. This can again be explained by the thermal elongation of the beams. It is remarkable that the membrane forces in the longitudinal direction $n_{x}$ of the system with $20^{\circ} \mathrm{C}$ edge beams are much smaller than in the system with rigid supports. The reason is that more load is transferred in transversal direction with edge beams, as seen on $n_{y}$, since these not only provide vertical support but also some horizontal restraint. It becomes clear that the force distribution in a slab depends heavily on the vertical deformation of the beams. It could also be seen that the horizontal restraint plays a major role, therefore, considering only single slab panels and neglecting the interaction with adjacent panels, as it is assumed in simple calculation models, clearly leads to wrong force distributions and failure modes. The part with the highest forces is not necessarily the centre of the slab in longitudinal direction. The location depends on the whole system.

This can be further proved by following example. The slab of Example 5-1 is expanded by a second panel connected at the longitudinal edge. All edge beams are assumed to reach $540^{\circ} \mathrm{C}$. The distribution of the membrane forces is shown in Figure 5.18. Again no clear compression ring can be found. The compressive forces above the intermediate beam are mainly the compressive part of the composite beam. In Figure 5.18 a section across the short spans at the slab centre shows the membrane forces in $\mathrm{x}$-direction $n_{x}$. The highest forces do not occur at the slab centre but in the first third of the short span.

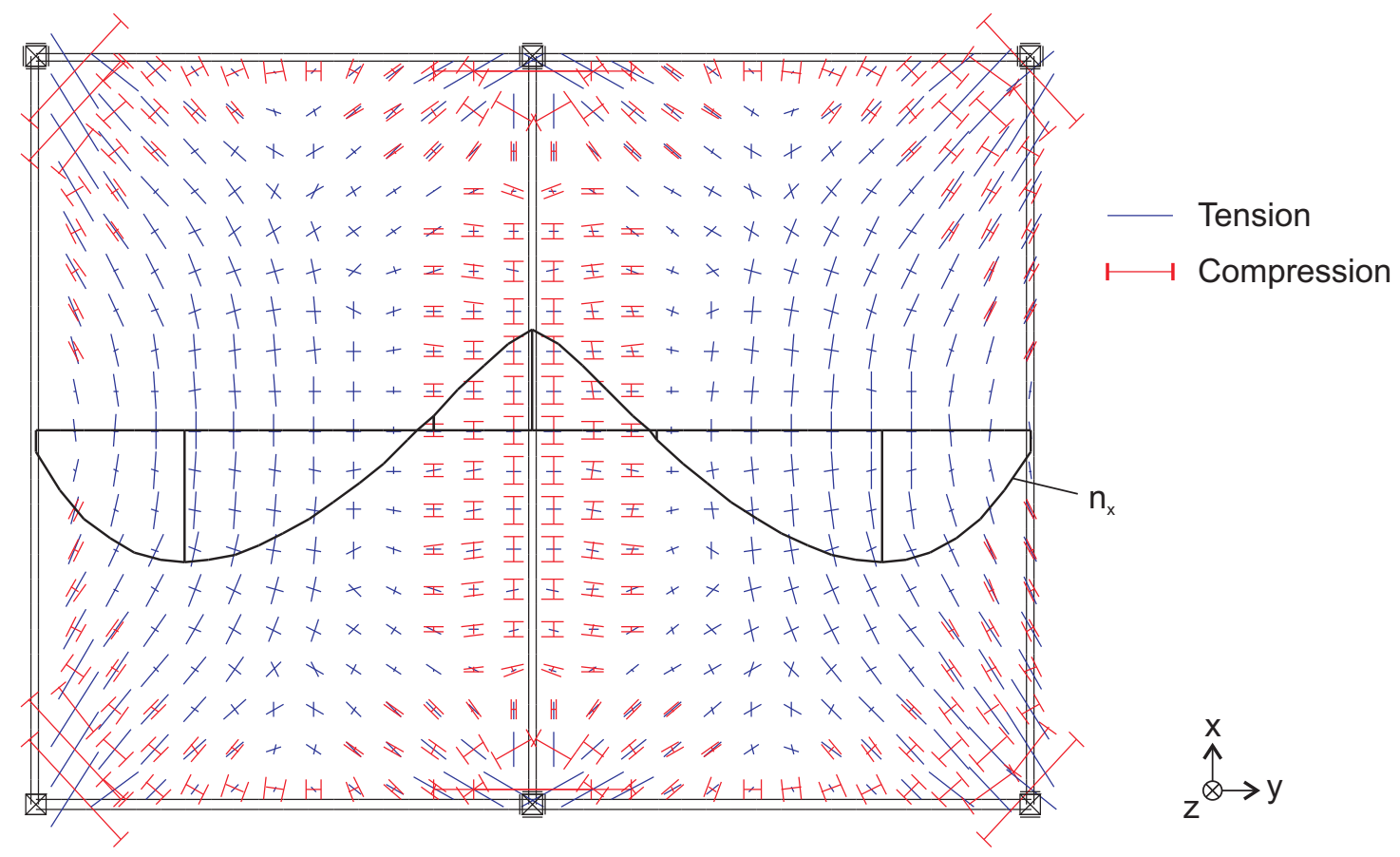

Fig. 5.18: Membrane forces of two adjacent slab panels

The influence of the edge beam stiffness on the slab becomes even clearer with the following example. On the one hand, the edge beams (EB) of the system above are modelled with $540^{\circ} \mathrm{C}$ and on the other hand with $20^{\circ} \mathrm{C}$. The results are assembled in Table 5.4. The deformation at the slab centre is similar with hot or cold edge beams. These are both much smaller than if only a single slab panel is considered. The deformation of the intermediate beam differs dramatically: $177 \mathrm{~mm}$ with a heated beam compared to $0 \mathrm{~mm}$ with rigid supports. The membrane forces at the slab centre are higher if the beams are hotter and softer. On the other hand, the forces above the intermediate beam are higher if the beams are colder and stiffer. The membrane force perpendicular to the intermediate beam $n_{y}$ and the bending moment $m_{y}$ are particularly higher with stiffer beams. 
Table 5.4: Comparison of results of two adjacent slab panels with different support conditions

\begin{tabular}{|l|c|c|c|c|c|c|}
\hline & \multicolumn{3}{|c|}{ Slab centre } & \multicolumn{3}{c|}{ Intermediate beam } \\
\hline & $w$ & $n_{x}$ & $n_{y}$ & $w$ & $n_{y}$ & $m_{y}$ \\
\hline & {$[\mathrm{mm}]$} & {$[\mathrm{kN} / \mathrm{m}]$} & {$[\mathrm{kN} / \mathrm{m}]$} & {$[\mathrm{mm}]$} & {$[\mathrm{kN} / \mathrm{m}]$} & {$[\mathrm{kNm} / \mathrm{m}]$} \\
\hline EB $20^{\circ} \mathrm{C}$ & 240 & 23.6 & 26.1 & 40 & 107 & -30.1 \\
\hline EB $540^{\circ} \mathrm{C}$ & 244 & 151 & 70.8 & 177 & 86.4 & -17.6 \\
\hline
\end{tabular}

To summarise, the edge beams must be included in a numerical model and cannot be neglected or replaced by rigid supports. Beam elements with uniform temperature distribution, thermal elongation and linear elastic material can be used. Bending hinges should be modelled at the ends of every beam. Two limiting cases should be considered: cold edge beams in order to get the highest forces at the intermediate beams and hot beams for the highest forces at the slab centre. 


\section{Design}

Previous chapters explain how concrete and composite slab systems in case of fire can be modelled very simply, taking into account membrane effects. The internal forces in the slabs are determined directly from the finite element model. In this chapter the determination of internal forces in the beams and member design will be explained. Generally, the available cross-section design methods given in the Eurocodes can be used. Full concrete slabs are designed according to DIN EN 1992-1-2 [27] and composite slabs and beams according to DIN EN 1994-1-2 [32]. The relevant sections of the Eurocodes will be explained and the parts of the structure which require special attention will be described. Additionally, some simplifications are made which follow the specifications of the Eurocodes but simplify the design procedure considerably.

\subsection{Design of the slabs}

Generally, each cross-section in the slab has to be designed for its corresponding internal forces. Finite element programs that are used for designing concrete slabs in practice such as Sofistik often include design routines which deliver required reinforcement amounts at each location. These routines mostly work only for ambient temperature design however. The design methods in this chapter are therefore chosen and modified such that ambient temperature design methods can be applied. If the required reinforcement is designed manually, it is sufficient to consider some locations with the highest stresses. These locations are usually the centre of the slab in both directions, above the intermediate beams and above the columns in both directions. They depend on many factors however, such as the geometry of the structure or non-uniform loading, so a general rule cannot be followed for all cross-section designs.

\subsubsection{Design of full concrete slabs}

In Annex B of DIN EN 1992-1-2 [27], two different simplified methods are given for designing reinforced concrete beams and slabs in case of fire. One of the methods, the $500^{\circ} \mathrm{C}$ isotherm method, must not be applied in Germany according to the national annex [28] and is therefore not used in this work. Annex B.2 provides the so called zone method which can be traced back to Kristian Hertz [38]. This method can only be applied for the standard fire curve. A cross-section is divided into several zones as shown in Figure 6.1. For each zone the mean temperature $\theta_{i}$ and the corresponding reduction coefficient for the concrete strength $k_{c}\left(\theta_{i}\right)$ are determined. A mean reduction coefficient $k_{c, m}$ and the depth of a damaged zone $a_{z}$ can be calculated as follows:

$$
\begin{aligned}
& k_{c, m}=\frac{1-0.2 / n}{n} \sum_{i=1}^{n} k_{c}\left(\theta_{i}\right) \\
& a_{z}=w\left[1-\frac{k_{c, m}}{k_{c}\left(\theta_{M}\right)}\right]
\end{aligned}
$$


Where:
$n \quad$ is the number of parallel zones in $w$;
$w \quad$ is in case of a slab the depth $h$;
$k_{c}\left(\theta_{M}\right) \quad$ is the reduction coefficient at point $\mathrm{M}$ which is at the top surface in case of a slab.

A cross-section with a reduced depth $h-a_{z}$ and a reduced compressive strength $k_{c}\left(\theta_{M}\right) \cdot f_{c, 20}$ is assumed and used for design. The strength of the reinforcement has to be reduced according to its temperature. All design methods that are available for ambient temperature can be applied to this reduced cross-section.

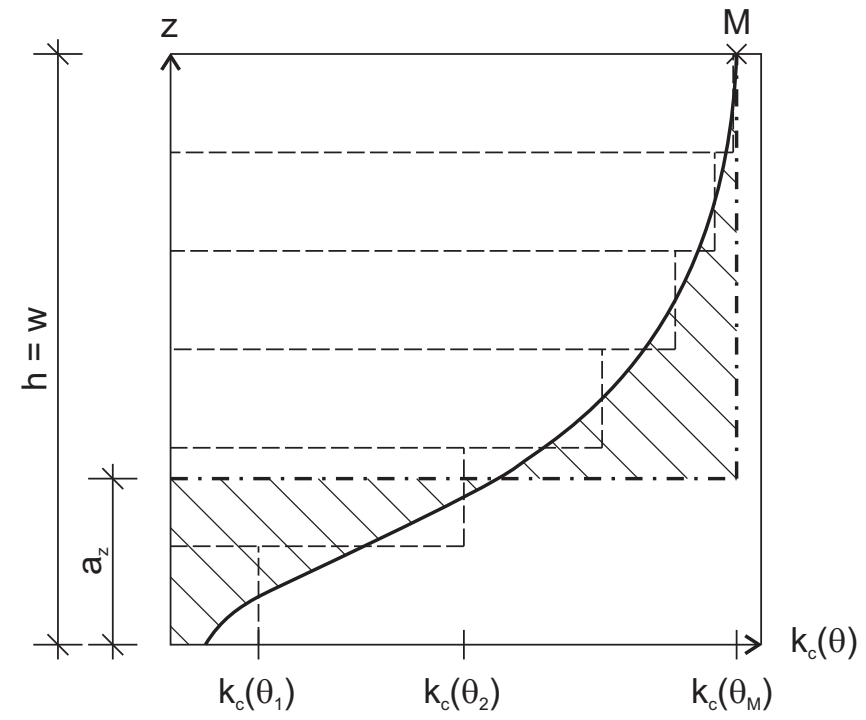

Fig. 6.1: Zone method

It is not clearly explained in the Eurocode [27] which concrete strength for the compression zone has to be used if the compression zone is at the heated surface. In [38] and [39] a more detailed description of the method and examples can be found where it is explained that the concrete strength at point $\mathrm{M}$ at the top surface of the slab as shown in Figure 6.1 must always be used. This becomes clear if $a_{z}$ is not considered as a damaged zone where the concrete cannot bear any stresses. As shown in Figure 6.1, the cross-section with a non-linear strength distribution (bold line) is replaced by an equivalent cross-section with the uniform strength at point $\mathrm{M}$ (dash-dot line). $a_{z}$ is determined such that the bearable stresses, that are missing for a uniform strength in the reduced cross-section, are the same amount as the bearable stresses within the area in $a_{z}$. The shaded areas in Figure 6.1 are equal in size. These assumptions are conservative for cross-sections with hogging moments. The resulting bearable force has a larger lever arm in the area $a_{z}$ than in the equivalent cross-section. The bending resistance of the real cross-section is therefore higher than the resistance of the equivalent cross-section.

In Figure B.5 of DIN EN 1992-1-2 [27] there are diagrams given for $a_{z}$ and $k_{c}\left(\theta_{M}\right)$ for different values of $w$ and times of the standard fire curve. These diagrams do however not agree with the equations given in the Eurocode. It is specified in the Eurocode that the cross-section shall be divided into at least three layers $(n \geq 3)$. Equation (6.2), that corresponds to Equation B.12 in DIN-EN-1992-1-2 [27] however, delivers different results for $a_{z}$ if a different number of layers $n$ in Equation (6.1) is used. As shown in Figure 6.2, the values for $a_{z}$ are higher than the values of Figure B.5 if five layers are used $(n=5)$ and are even more higher with three layers, which is not shown in Figure 6.2. With nine layers $(n=9)$ the values calculated with the equations are lower than the values of Figure B.5. Even if very many layers 


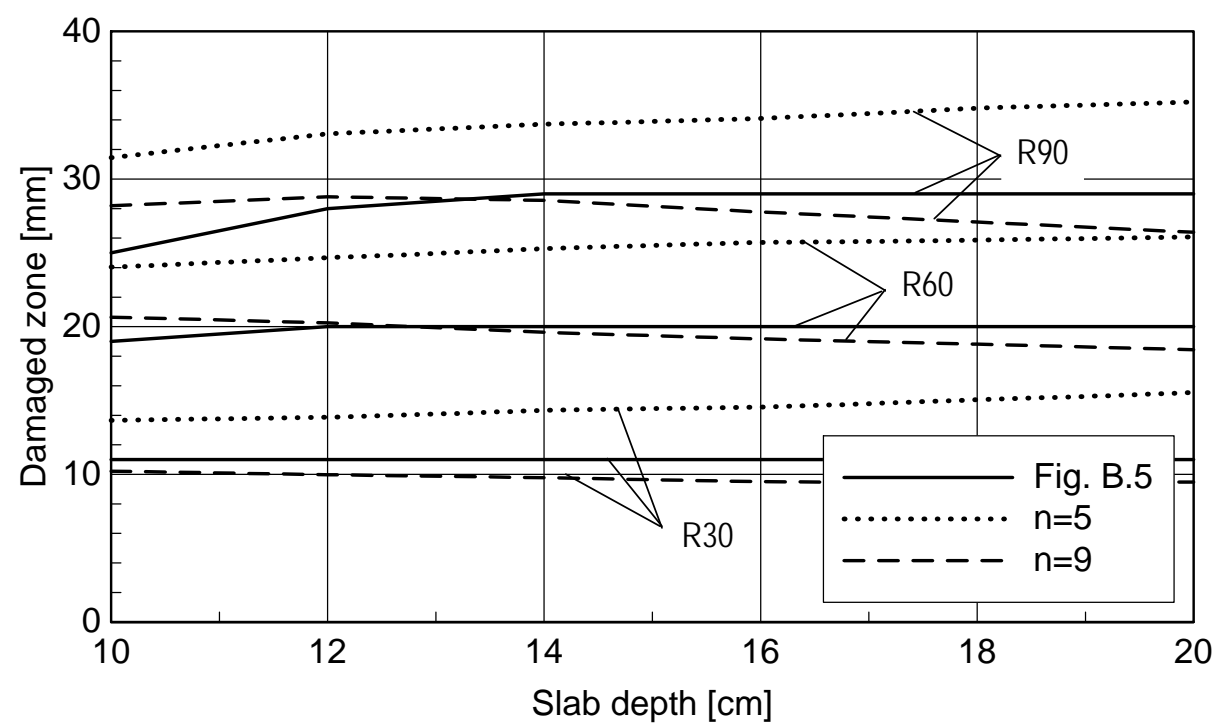

Fig. 6.2: Comparison of damaged zone $a_{z}$ according to DIN EN 1992-1-2, Annex B, Figure B.5 and Equation B.12 [27]

are used $(n=50)$, the values of Figure B.5 cannot be reproduced. The reason is presumably that different temperature profiles were used for creating Figure B.5 than in this work. The profiles used in this work, however, are in agreement with Figure A.2 of DIN-EN-1992-1-2 [27] and are therefore assumed to be correct according to the latest regulations. Possibly the profiles used for Figure B.5 were calculated according to an older version of the Eurocode. Since the values vary in a certain range, simplified the even values shown in Table 6.1 are suggested to be used.

Table 6.1: Damaged zone $a_{z}$ and minimum distance of the top reinforcement from bottom surface $z_{b}$

\begin{tabular}{|c|c|c|}
\hline & $a_{z}$ & $z_{b}$ \\
\hline & {$[\mathrm{mm}]$} & {$[\mathrm{mm}]$} \\
\hline R30 & 10 & 16 \\
\hline R60 & 20 & 31 \\
\hline R90 & 30 & 43 \\
\hline
\end{tabular}

A further simplification is given in this table. If the reinforcement in the slab is placed at a distance of at least $z_{b}$ from the bottom surface of the slab, it remains lower than $400^{\circ} \mathrm{C}$ and its full strength can be assumed. This is mostly the case for the top reinforcement. If a bottom reinforcement is required, which is mostly not the case, the steel temperature can be found for instance with Figure A.2 of DIN-EN-19921-2 [27] or Table D.5 of DIN EN 1994-1-2 [32] and the steel strength has to be reduced by the coefficients given in Table 3.2 of DIN-EN-1992-1-2 [27]. The bottom reinforcement is assumed to be at its original location, even if this is outside of the reduced cross-section. The concrete strength for the cross-section design is in most cases the full strength at $20^{\circ} \mathrm{C} f_{c, 20}$. Only in the cases given in Table 6.2, the top surface of the slab reaches temperatures, which are high enough, that the concrete strength has to be reduced by the factor $k_{c}\left(\theta_{M}\right)$. These values are in good agreement with Figure B.5 of DIN-EN-1992-1-2 [27].

To summarise, the design of full concrete slabs only requires a check that the distance of the reinforcement from the bottom surface is sufficient according to Table 6.1 and if the concrete strength has to be 
Table 6.2: Reduction coefficient $k_{c}\left(\theta_{M}\right)$ for concrete at point M

\begin{tabular}{|c|c|c|}
\hline & $h$ & $k_{c}\left(\theta_{M}\right)$ \\
\hline & {$[\mathrm{cm}]$} & {$[-]$} \\
\hline R60 & 10 & 0.994 \\
\hline R90 & 10 & 0.956 \\
\hline R90 & 12 & 0.990 \\
\hline
\end{tabular}

reduced according to Table 6.2. The cross-section depth is reduced by the values of Table 6.1 and all cross-section design methods for ambient temperature can be used with the remaining cross-section.

\subsubsection{Design of composite slabs}

Methods for calculating the load bearing capacity of composite slabs in case of fire can be found in Annex D of DIN EN 1994-1-2 [32]. These methods are again only valid for the standard fire curve and only for fire resistance classes above R60. They can be traced back to Cornelis Both [10]. Only methods for sagging and hogging moments are given but none for combined loading with axial and shear forces. The method for the hogging moment resistance however is very similar to the zone method described in the previous section and can therefore be adopted for combined loading, particularly against the background that mainly tensile membrane forces and hogging moments occur in the slabs considered in this work. Like for the zone method for full concrete slabs, the cross-section is reduced, as shown in Figure 6.3 with the dashed lines, and design methods for ambient temperatures are used for the remaining parts. The contribution of the steel decking to the load bearing capacity is neglected.

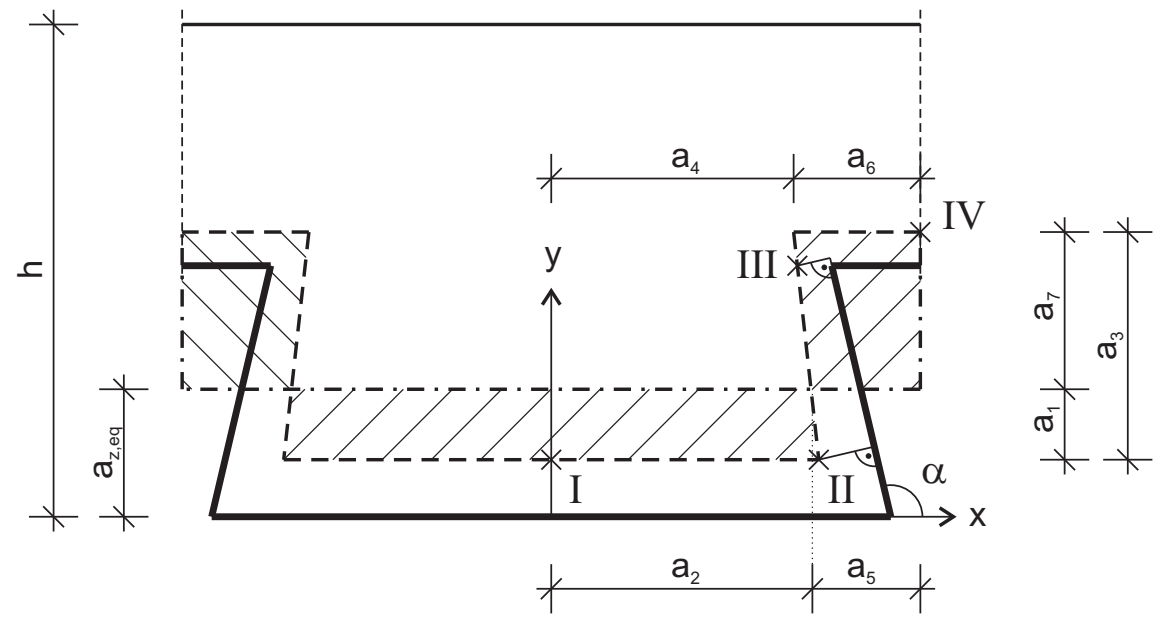

Fig. 6.3: Reduced cross-section according to DIN EN 1994-1-2, Annex D [32] and equivalent rectangular cross-section

The areas that are neglected are specified by the points I to IV. Equations are given in DIN EN 19941-2, Annex D [32] for determining the coordinates $\mathrm{x}$ and $\mathrm{y}$ of these points. It should be remarked that for a re-entrant trough profile steel sheeting the angle $\alpha$ generally must be assumed as shown in Figure 6.3. The angle calculated with Equation (D.10) in the Eurocode leads to the wrong coordinates of the points I to IV for this kind of steel sheeting. The problem with the method in the Eurocode is that the remaining cross-section is not rectangular and therefore cannot be used in design routines that are implemented in ordinary finite element programs such as Sofistik. The non-rectangular cross-section is therefore replaced 
by an equivalent rectangular cross-section as shown in Figure 6.3 with the dash-dot line. The distance $a_{1}$ is chosen such that the areas of the equivalent and the original cross-section are equal. This assumption is conservative since the section modulus is lower at the equivalent cross-section. The distances as shown in Figure $6.3 a_{2}$ to $a_{7}$ can be determined as follows:

$$
\begin{aligned}
& a_{2}=x_{I I}-\frac{a_{1}}{a_{3}}\left(x_{I I}-x_{I I I}\right) \\
& a_{3}=y_{I I I}-y_{I} \\
& a_{4}=x_{I I}-\frac{y_{I V}-y_{I I}}{a_{3}}\left(x_{I I}-x_{I I I}\right) \\
& a_{5}=x_{I V}-a_{2} \\
& a_{6}=x_{I V}-a_{4} \\
& a_{7}=y_{I V}-y_{I I}-a_{1}
\end{aligned}
$$

It will be assumed that the shaded areas in Figure 6.3 are equal in size:

$$
\frac{x_{I I}+a_{2}}{2} a_{1}=\frac{a_{5}+a_{6}}{2} a_{7}
$$

Equation (6.9) leads to a long quadratic equation or can be solved numerically in order to determine $a_{1}$. The total distance from the bottom of the composite slab to the bottom of the equivalent cross-section will be called equivalent damaged zone and denoted by $a_{z, e q}$ like the damaged zone in the zone method for full concrete slabs.

$$
a_{z, e q}=y_{I}+a_{1}
$$

In contrast to the zone method, the negligible parts are not determined by homogenising the bearable stresses as explained in the previous section. The hogging moment resistance of the real cross-section is equal to the resistance of a reduced cross-section of uniform concrete strength. This means that the shape of the reduced composite slab cross-section does not depend on the slab depth but on the available reinforcement. The depth of the damaged zone $a_{z, e q}$ of an equivalent rectangular cross-section is charted in Figure 6.4 with the bold lines as a function of the reinforcement amount on the example of a Holorib HR51 slab. It can be seen that the damaged zone is smaller if less reinforcement is available. This fact complicates the design procedure in practice considerably. It means that the required reinforcement amount has to be determined iteratively. A reinforcement amount has to be estimated, the corresponding reduced cross-section has to be determined and it has to be checked if the estimated cross-section can bear the internal forces determined by the finite element analysis. This procedure has to be repeated until the right reinforcement amount is found. Furthermore, it has to be performed for every cross-section in a slab since the internal forces vary and the required reinforcement amount is higher above intermediate beams and columns than at the slab centre. The method therefore needs to be simplified further in order to achieve a more useful approach.

The slab cross-section is assumed to be rectangular with an effective depth according to DIN EN 19941-2, Annex D.4 [32] as shown in Figure 6.5. This effective depth is reduced by a damaged zone $a_{z}$ for full concrete slabs as derived in the previous section. The effective depth depends only on the geometry of the steel sheeting and the damaged zone depends only on the fire resistance class. This means that for the 


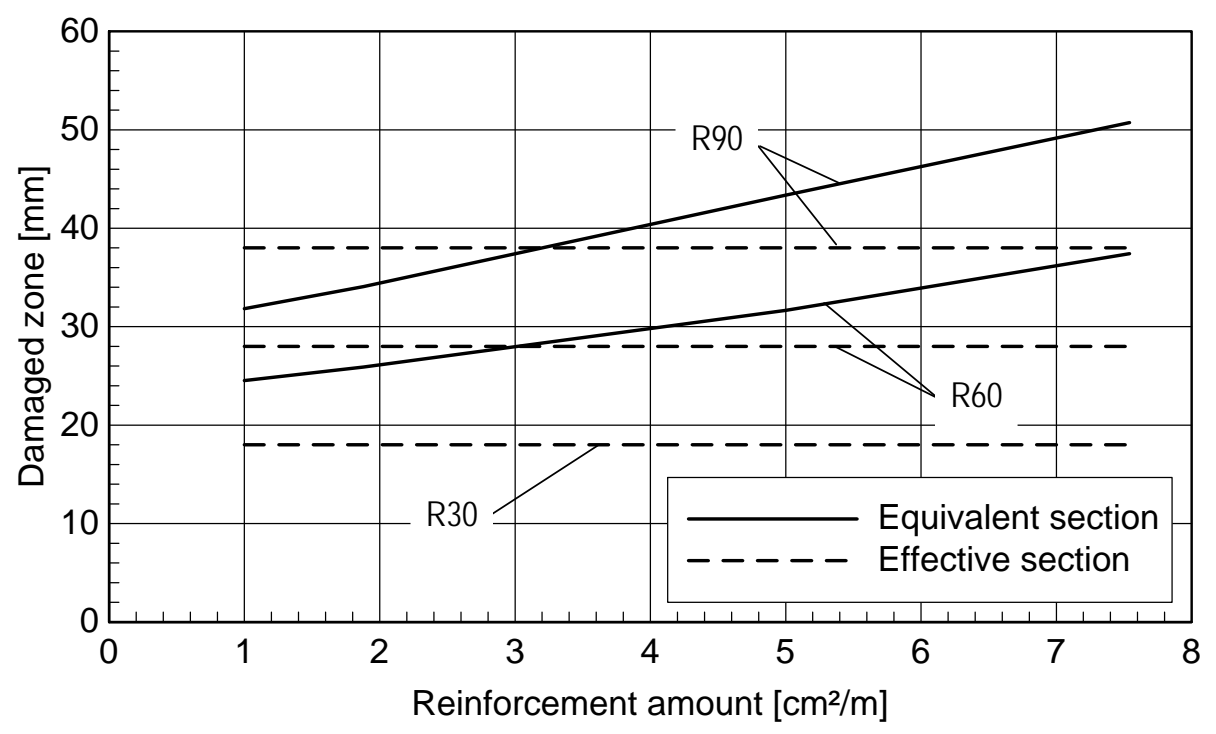

Fig. 6.4: Damaged zone of a Holorib HR51 slab with equivalent cross-section $a_{z, e q}$ and effective depth $\operatorname{method} a_{z, e f f}$

whole slab only one thickness needs to be used and the required reinforcement amount can be designed for the same cross-section at every location. This method can also be applied for a fire resistance class R30. For the Holorib profile the resulting damaged zone $a_{z, e f f}$ for the effective thickness method is shown in Figure 6.4 with the dashed lines. It can be seen that both methods deliver the same results for a reinforcement amount of about $3 \mathrm{~cm}^{2} / \mathrm{m}$. For a Cofraplus 60 profile, this intersection point lies even above $6 \mathrm{~cm}^{2} / \mathrm{m}$. Since the equivalent rectangular cross-section method already contains conservative assumptions and the reinforcement amounts will mostly not be much higher than $3 \mathrm{~cm}^{2} / \mathrm{m}$, the effective thickness method can be safely applied on the slabs considered in this work and does not disagree with the regulations in the Eurocode. The values for the damaged zone $a_{z, \text { eff }}$ of Holorib HR51 and Cofraplus 60 slabs determined with the effective thickness method are shown in Table 6.3.

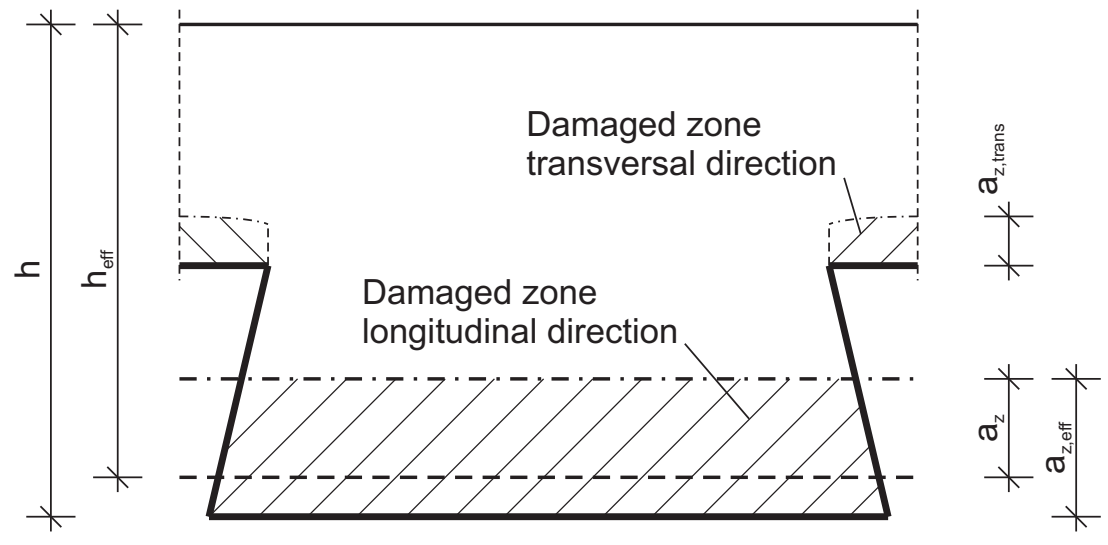

Fig. 6.5: Damaged zone of effective thickness method

If sagging moments occur, the method of DIN EN 1994-1-2, D.2 [32] can generally be used. The contribution of the steel sheeting should not be taken into account since it could debond in a fire. In the German national annex [33] it is only allowed to be taken into account if the bonding can be proved. If any sagging moments should occur, these will be very small and it will presumably be sufficient 
considering a cross-section with the top reinforcement and the concrete above.

In the transversal direction, only the concrete above the steel decking should be taken into account since the weakest cross-section is above the ribs. No approaches to determine the damaged zone in transversal direction $a_{z, \text { trans }}$ are currently available. This zone is very small, as shown in Figure 6.5 since the temperatures in the slab above the top flange of the steel decking are much lower than above the bottom flange. This is true particularly for a re-entrant trough profile steel sheeting. The same values for the damaged zone $a_{z}$ could be used as for full concrete slabs but this assumption is very conservative. A pragmatic approach is suggested that the damaged zone in transversal direction $a_{z, \text { trans }}$ is $50 \%$ of $a_{z}$ for full concrete slabs. In order to be able to provide more scientifically based approaches, further research work including experimental validation is necessary. For the application in this work, the suggested assumtions are sufficient.

Table 6.3: Damaged zone in longitudinal $a_{z, \text { eff }}$ and transversal $a_{z, \text { trans }}$ direction of Holorib HR51 and Cofraplus 60 slabs for the effective thickness method

\begin{tabular}{|c|c|c|c|}
\hline & HR51 & Cof. 60 & Trans. \\
\hline & $a_{z, \text { eff }}$ & $a_{z, \text { eff }}$ & $a_{z, \text { trans }}$ \\
\hline & {$[\mathrm{mm}]$} & {$[\mathrm{mm}]$} & {$[\mathrm{mm}]$} \\
\hline R30 & 18 & 45 & 5 \\
\hline R60 & 28 & 55 & 10 \\
\hline R90 & 38 & 65 & 15 \\
\hline
\end{tabular}

To summarise, for the design of composite slabs in longitudinal direction, a full concrete slab with an effective thickness according to DIN EN 1994-1-2, D.4 [32] shall be considered. With this cross-section the method for full concrete slabs as described in Section 6.1.1 can be used. In transversal direction only the concrete above the ribs should be taken into account. This cross-section is reduced by $50 \%$ of the damaged zone $a_{z}$ of full conrete slabs and again the method of Section 6.1.1 can be used.

\subsection{Design of the beams}

The beams are modelled in this work with a linear-elastic material as described in Section 5.3. This approach was chosen in order to avoid material non-linear analyses which require very long computing times. As a consequence, the model delivers correct internal forces in the slab but not in the beams. These need to be determined separately. The approach, where geometrical non-linearities need to be taken into account but no material non-linearities, can be justified by following Example 6-1. A composite beam is considered as shown in Figure 6.6. The beam offers a length of $7.5 \mathrm{~m}$ and is exposed to the standard fire for sixty minutes from below. It is horizontally fixed at both ends by the nodes of the concrete flange but not at the nodes of the steel beam as explained in Section 5.3. The steel section consists of an IPE 240 profile in S235 and is protected against heating for a fire resistance class R60 which leads to a temperature in the steel of $540^{\circ} \mathrm{C}$. The temperature is assumed to be uniform which reduces the Young's modulus of the steel to $E_{\theta}=101640 \mathrm{MPa}$ and the yield strength to $f_{y, \theta}=154 \mathrm{~N} / \mathrm{mm}^{2}$ and implies a substitute thermal loading of $\varepsilon_{\theta, s u b s}=7.40 \%$. The concrete flange consists of a full concrete slab C20/25 with a depth of $12 \mathrm{~cm}$ and an effective width of $1.875 \mathrm{~m}$. The temperature distribution in the concrete leads to a substitute thermal loading of $\varepsilon_{\theta, s u b s}=1.576 \%$ and $\kappa_{\theta, \text { subs }}=50.58 \mathrm{~km}^{-1}$ from Table 4.6. Since the concrete is under compression in this example, the Young's modulus is only reduced by the 

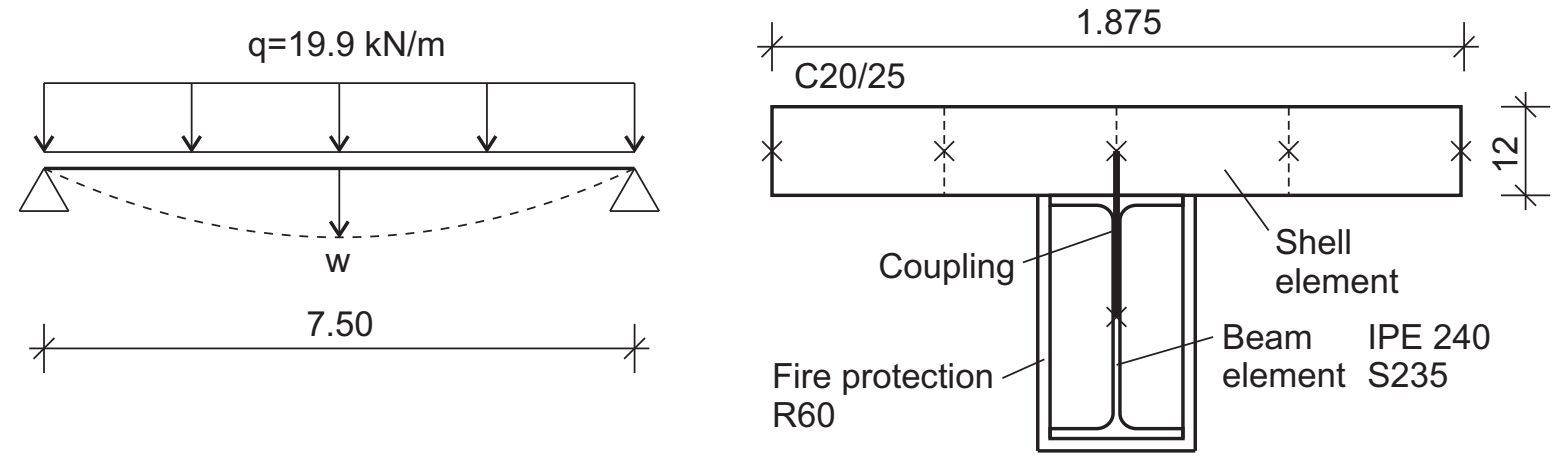

Fig. 6.6: System (left) and cross-section (right) of Example 6-1: load bearing characteristics of composite beam

factor for cross-section softening $k_{E, \theta, \text { mean }}=0.480$ from Table 5.1. The sagging moment resistance of the composite beam can be calculated with the method in DIN EN 1994-1-2, Annex E.1 [32]. The plastic resistance of the steel section $N_{p l, a, R d, f i}=602 \mathrm{kN}$ is simplified by assuming a uniform temperature. This leads to a bending resistance of $M_{f i, R d}^{+}=140 \mathrm{kNm}$ and a maximum bearable uniform load of a simply supported beam of $q_{R d, f i}=19.9 \mathrm{kN} / \mathrm{m}$ which is applied to the system. The input file can be found in Annex B.5.

In Example 6-1a only the mechanical load is applied. The material strength and stiffness are reduced due to heating but no thermal elongation is taken into account. A simplified bi-linear stress-strain relationship is used for the steel section with a horizontal yielding part. In this calculation only material non-linearities are taken into account, no geometrical non-linearities. The load level $\eta$ of the steel section is close to $100 \%$ as shown in Table 6.4. It is calculated by a plastic cross-section design according to DIN EN 1993-1-2 [29]. This example shows that the model delivers correct results for the plastic calculation of composite beams.

Table 6.4: Comparison of results of Example 6-1

\begin{tabular}{|c|c|c|c|c|c|}
\hline & & \multicolumn{2}{|c|}{ non-linear } & $w$ & $\eta$ \\
\hline Example & $\varepsilon_{\theta}+\kappa_{\theta}$ & mat & geo & {$[\mathrm{mm}]$} & {$[-]$} \\
\hline 6-1a & - & + & - & 46.1 & 0.95 \\
\hline 6-1b & + & + & + & 201 & 0.99 \\
\hline 6-1c & + & - & + & 199 & 2.28 \\
\hline
\end{tabular}

In Example 6-1b both mechanical and thermal loading are applied. Material non-linearities are taken into account in addition to geometrical non-linearities. This model best represents the real behaviour of the beam. In Table 6.4 it can be seen that the load level $\eta$ of the steel section is again very close to $100 \%$. The restraint forces, which occur due to the different thermal elongation of the steel beam and the concrete flange, yield out at the ultimate limit state. The load bearing capacity of the composite beam is the same if restraint forces are taken into account or are neglected. For the same reason, shrinkage does not need to be considered when determining the load bearing capacity of composite beams under ambient temperature, although it causes restraint forces like the thermal elongation. It only influences the deformation of the beams. This can also be seen in Example 6-1b. The vertical displacement at midspan $w$ is considerably larger than in Example 6-1a without thermal elongation but the load bearing capacity is the same. 
The model for Example 6-1c is set up as suggested in Section 5.3. Again both mechanical and thermal loadings are taken into account. The material is assumed to be linear-elastic, only geometrical nonlinearities are considered. The load level of the steel section $\eta$ is $228 \%$, much higher than theoretically bearable since no material non-linearities are taken into account and thus no load transfer occurs. The vertical deformation at midspan $w$ is the same as in Example 6-1b however where material non-linearities are included.

This example shows that it is sufficient to consider only geometrical non-linearities. Beam deformations, which largely influence the internal forces in the slabs as discussed in Section 5.3, are taken into account realistically. This approach enables the use of a linear-elastic material which ensures small computing times. The design of the beams at the ultimate limit state has to be performed separately. Since the composite beams can be considered to be simply supported as described in Section 5.3, the design is relatively simple. DIN EN 1994-1-2 [32] provides cross-section design methods for all required cases: bending resistance including partial shear connection, vertical shear resistance, resistance of shear studs etc.

The only question is how the loads are distributed to the beams in case of fire. For two-way spanning slabs under ambient temperature, the load distribution is generally assumed as shown in Figure 6.7. The angles of the assumed areas depend on whether the considered edge can rotate freely or is clamped due to the continuity of the slab. This approach is justified if a slab is rigidly supported at the edges or the edge

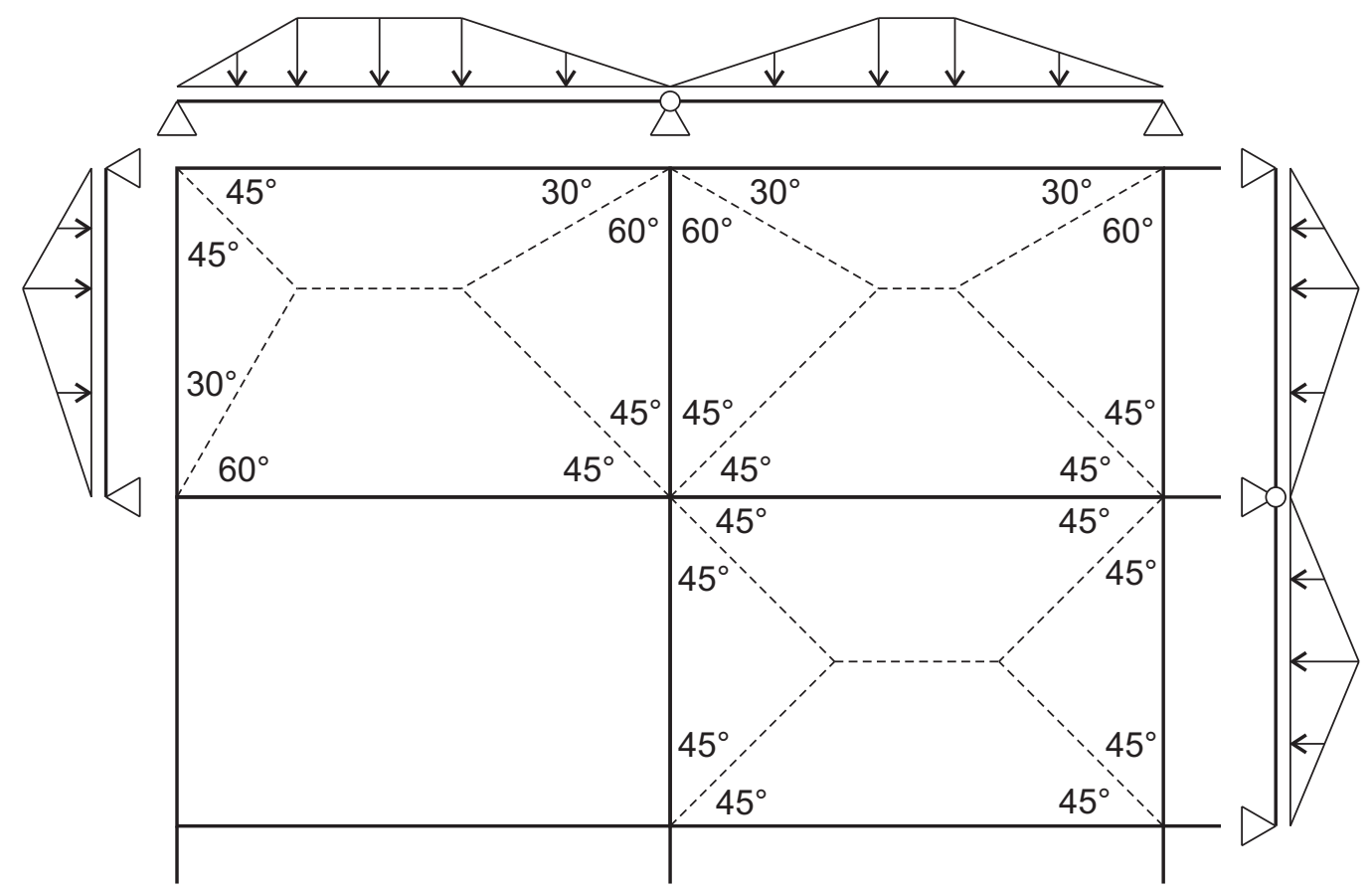

Fig. 6.7: Load distribution to edge beams according to [42]

beams are relatively stiff and only offer small deformations. Figure 6.8 illustrates such load distributions on a beam. The beam is a longitudinal edge beam of a single slab panel like in Example 5-1 as shown in Figure 5.4. The arrows in the upper part of Figure 6.8 show the coupling forces in the vertical direction between the concrete slab and the steel beam. These qualitatively represent the load transferred to the beam. On the left hand side of Figure 6.8 the steel beam has a temperature of $20^{\circ} \mathrm{C}$ and the slab is only loaded with a uniform mechanical load. It can be seen that the load distribution shows the trapezoidal shape according to the assumption in Figure 6.7. 

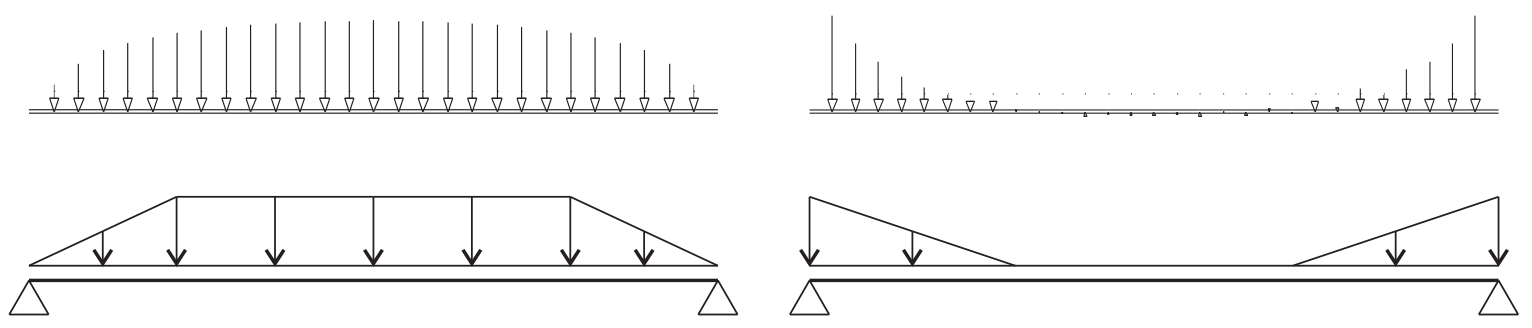

Fig. 6.8: Load distribution on longitudinal edge beam at $20^{\circ} \mathrm{C}$ (left) and $540^{\circ} \mathrm{C}$ (right)

The right hand side of Figure 6.8 shows the load distribution if the slab is exposed to the standard fire from below for ninety minutes and the steel beam reaches $540^{\circ} \mathrm{C}$. It can be seen that the load distribution changes considerably. The forces almost disappear at midspan and concentrate at the ends of the beam. The shape of the load distribution can be assumed triangular in this case. The reason for this load redistribution can be seen in Figure 6.9. It shows the membrane forces in the considered slab. It can be seen that large tensile forces run diagonally from the centre of the slab to the corners. This means that the loads are transferred directly from the slab to the columns which provide a much stiffer support than the beams.
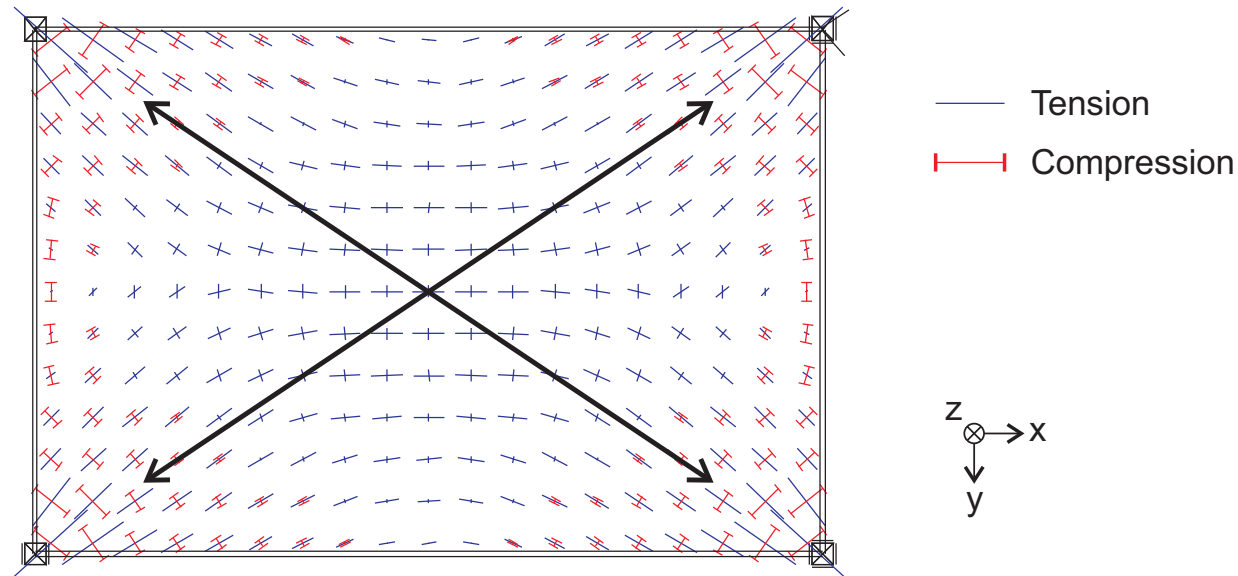

Fig. 6.9: Membrane forces with edge beams $540^{\circ} \mathrm{C}$, load transfer to columns

It has been shown that the trapezoidal load distribution is a good assumption if the supporting edge beams remain cold and stiff. If these become hot and lose stiffness, a triangular load distribution can be assumed. Generally, both cases have to be considered when designing the beams since it is unknown whether the steel beams reach the maximum temperature. The height of the loading for the hot case is difficult to quantify. It changes considerably with the stiffness of the beams. The conservative approach is therefore proposed that the trapezoidal load distribution should be used on the hot beams with reduced strength.

In the report of the DASt project [42] a simplified method for designing the edge beams is provided. The primary edge beams are usually sufficiently strong since the load that these have to carry in case of fire is much smaller than under ambient temperature. On the one hand, the safety factors for the loading are much smaller in case of fire. On the other hand, the load on the slab is transferred more directly to the secondary edge beams in case of fire, contrary to ambient temperature where the loads are transferred over the unprotected secondary beams to the primary edge beams. The secondary edge beams have to carry a larger part of the uniform load on the slab in case of fire than under ambient temperature. The 
load level of the bending moments in case of fire at midspan must be equal to or smaller than $100 \%$ :

$$
\frac{M_{E d, f i}}{M_{R d, f i}} \leq 1.0
$$

The moments in case of fire can be expressed by the moments under ambient temperature [42]:

$$
\begin{aligned}
& M_{E d, f i}=\alpha \eta_{f i} M_{E d} \\
& M_{R d, f i}=\gamma M_{R d}
\end{aligned}
$$

Where:

$$
\begin{aligned}
& \alpha=\frac{M_{E d, f i}}{\eta_{f i} M_{E d}} \approx \frac{b}{b_{1}} \\
& \eta_{f i}=\frac{q_{E d, f i}}{q_{E d}} \leq 0.65 \\
& \gamma=\frac{M_{R d, f i}}{M_{R d}} \approx \frac{\gamma_{M 0}}{\gamma_{M, f i}} k_{y, \theta} \approx 0.656 \text { if } \theta_{a}=540^{\circ} \mathrm{C}
\end{aligned}
$$

With Equation (6.11) to (6.16) the maximum allowable load level for the secondary edge beams under ambient temperature can be found:

$$
\frac{M_{E d}}{M_{R d}} \leq \frac{\gamma}{\alpha \eta_{f i}}
$$

The reduction factor $\eta_{f i}$ is the ratio of the design values of the applied uniform load on the slab under ambient temperature $q_{E d}$ to the load in the fire case $q_{E d, f i}$. A conservative simplified value $\eta_{f i}=0.65$ is proposed in DIN EN 1993-1-2, 2.4.2 [29]. The coefficient $\gamma$ is the ratio of the design values of the bending resistance in case of fire $M_{R d, f i}$ to the bending resistance under ambient temperature $M_{R d}$. If both partial safety factors $\gamma_{M 0}$ and $\gamma_{M, f i}$ are equal to 1.0 and the plastic neutral axis of the composite cross-section lies within the concrete slab, $\gamma$ is approximately equal to the reduction factor for the steel strength $k_{y, \theta}$. The coefficient $\gamma$ equals 0.656 if the steel beam reaches $540^{\circ} \mathrm{C}$. The coefficient $\alpha$ is the ratio of the design values of the bending moments in case of fire $M_{E d, f i}$ to the bending moment under ambient temperature $M_{E d}$ multiplied with the factor $\eta_{f i}$ for the reduced loading in case of fire. It shows the difference between the bending moments in the secondary edge beam with and without unprotected secondary beams if in both cases the loading in case of fire $q_{E d, f i}$ is applied. If it is assumed that the
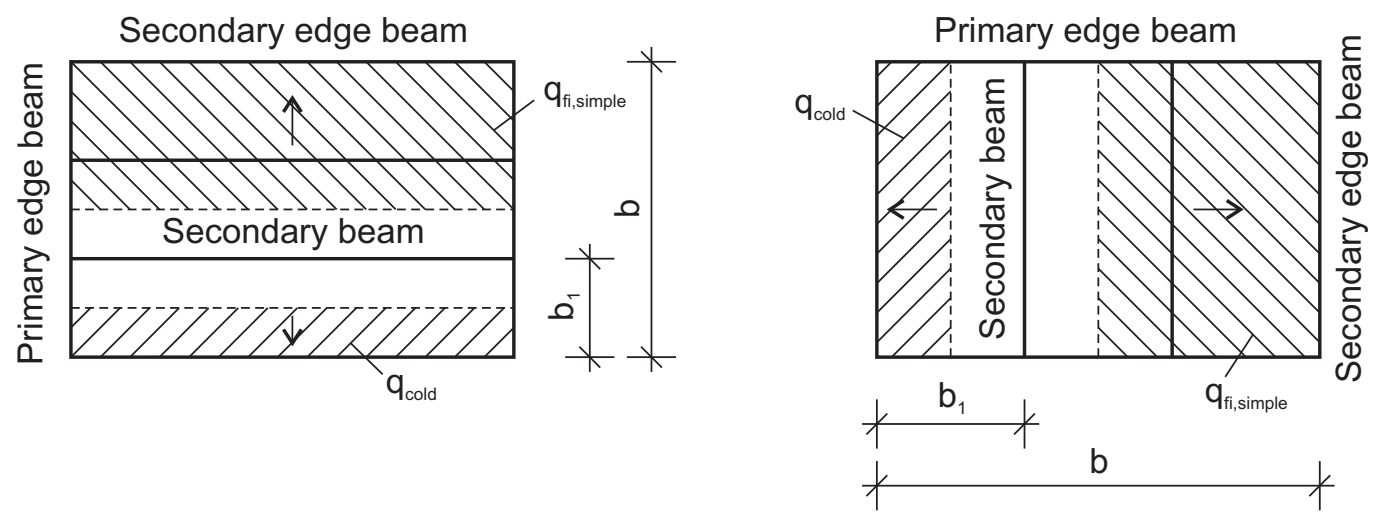

Fig. 6.10: Notation of coefficients $b$ and $b_{1}$ for simplified edge beam design according to [42] 
loading conservatively acts on the beams as a uniform line load, $\alpha$ can be replaced by the ratio of the primary beam length $b$ to the spacing of the secondary beams $b_{1}$ as shown in Figure 6.10.

The advantage of this approach is that the design of the secondary edge beams can be performed simply by considering the load level under ambient temperature. With the simplifications in Equations (6.14) to (6.16), Equation (6.17) leads to very simple values. For one secondary beam inside the slab panel it becomes:

$$
\begin{aligned}
& \alpha=\frac{b}{b / 2}=2 \\
& \frac{M_{E d}}{M_{R d}} \leq \frac{\gamma}{\alpha \eta_{f i}}=\frac{0.656}{2 \cdot 0.65}=0.50
\end{aligned}
$$

And for two secondary beams:

$$
\begin{aligned}
& \alpha=\frac{b}{b / 3}=3 \\
& \frac{M_{E d}}{M_{R d}} \leq \frac{\gamma}{\alpha \eta_{f i}}=\frac{0.656}{3 \cdot 0.65}=0.34
\end{aligned}
$$

These values are very conservative as can be seen in Figure 6.10. The larger shaded areas show the areas of the load which is assumed to be transferred to the secondary edge beams. These areas are much larger than they were in reality as shown in Figure 6.7. The load level of the secondary beams under ambient temperature however is often very small since deformation limits often govern the design. The values in Equation (6.19) and (6.21) can often be satisfied and no further calculation is therefore necessary.

Regulations for the design of connections between protected composite beams and columns are given in DIN EN 1994-1-2, 5.4 [32]. Basically, a connection is sufficiently protected if the same fire protection is applied as that of the member transmitting the actions, and the connection load ratio is less than or equal to that of the beam. Since the protected edge beams of membrane action slabs are ordinary composite beams, no adjustments to the regulations in the Eurocode are necessary. The connections between the unprotected secondary beams and the protected primary beams do not need to transfer loads and can therefore be left unprotected for load bearing reasons. Heat can be conducted from the unprotected into the protected beams however and the temperature in the protected beams can therefore become higher and the load bearing capacity lower than expected. The former fire protection norm in Germany DIN 4102-4 [19] demands in this case that fire protection shall be applied to a length of $30 \mathrm{~cm}$ on the unprotected members. The SCI design guide [44] proposes that the whole connection can be left unprotected. This proposal was adopted in the FRACOF design guide [48]. The investigations on which this proposal was based were not specified. As long as no investigations on this issue are available, it is proposed that the regulation of DIN 4102-4 [19] should be applied.

To summarise, for the design of the edge beams it is sufficient for only the mechanical loading to be taken into account. The restraint forces due to heating can be neglected since these have no effect on the load bearing capacity at the ultimate limit state. The load distribution on the beams can be assumed in the same way as under ambient temperature considering the slab panels without unprotected secondary beams as shown in Figure 6.7. With Equation (6.17) the design can be performed by simply considering the load level under ambient temperature. Conservative values for this allowable load level were provided. 


\section{Validation on fire tests}

In the previous chapters, a method was proposed which allows a very simple design of concrete and composite slab systems for the fire case. In this chapter, the method will be validated on experimental data. Validating the method on fire tests is partly difficult. The proposed method considers the ultimate limit state just before the structure collapses, however, this state is not reached in most large scale tests. Since the available furnaces for fire tests with oil or gas burners are usually too small for testing complete slab systems, temporary furnaces have to be erected and wooden cribs need to be used for the fire loading. The tests stop automatically as soon as the available fire load is exhausted and cannot be extended until the specimen collapses. Only the FRACOF test could be run until the slab failed since the furnace in this test was heated by burners. The mechanical loading also cannot be increased until failure during a large scale test since mostly dead loads such as sand bags have to be used. The test arrangements are usually too large for testing frames with adjustable hydraulic cylinders. Even if structural collapse occurs, such as at the test in Mokrsko (CZ) in 2008, the reason for the failure is difficult to reconstruct when the entire structure is destroyed.

The results of large scale tests furthermore include many uncertainties which complicate the validation of simulations or design methods. Non-uniform combustion of the fire load or uneven foaming of the intumescent coating can lead to non-uniform temperature development in the structural members for instance. This uncertainty can be reduced by evenly distributed thermocouples but the amount of measuring points is limited. Other uncertainties include measuring inaccuracies, statistical distribution of material properties or effects that cannot be measured during a fire test such as cracking of the concrete or slip in the shear connection between the steel beams and the slabs.

These explanations shall clarify that data of large scale fire tests should be used for validating numerical models and design methods with caution. Test results cannot necessarily be reproduced exactly due to many uncertainties in the test data. This cannot be avoided but it is nonetheless indispensable to validate design methods on test data in order to be sure that the assumptions are correct. Three tests are used for validation in this work: the two tests performed in Munich in 2010 and the test within the FRACOF project that was conducted in 2008. The test arrangements and most important results were described in Chapter 3. In this chapter the modelling of the tests is explained and the results of the simulations are compared with the test data.

\subsection{First Munich fire test}

The slab at the first Munich test did not collapse and the ultimate limit state was not reached, therefore, another time needs to be chosen to validate the proposed method. 40 minutes after test start will be considered since the system was most heavily loaded at this time. The gas temperatures in the furnace increased until about 40 minutes and started to fall again afterwards as shown in Figure 3.4. The boiling away of the uncombined water in the concrete was finished at this time and an even temperature distri- 
bution was reached, which can be seen in Figure 3.5. The temperatures in the steel beams reached their maximum after 40 minutes as shown in Figure 3.6 and Figure 3.7. Afterwards these started to cool down and the steel regained strength. For this reason, the system was weakest at this time although the largest deformations occurred later.

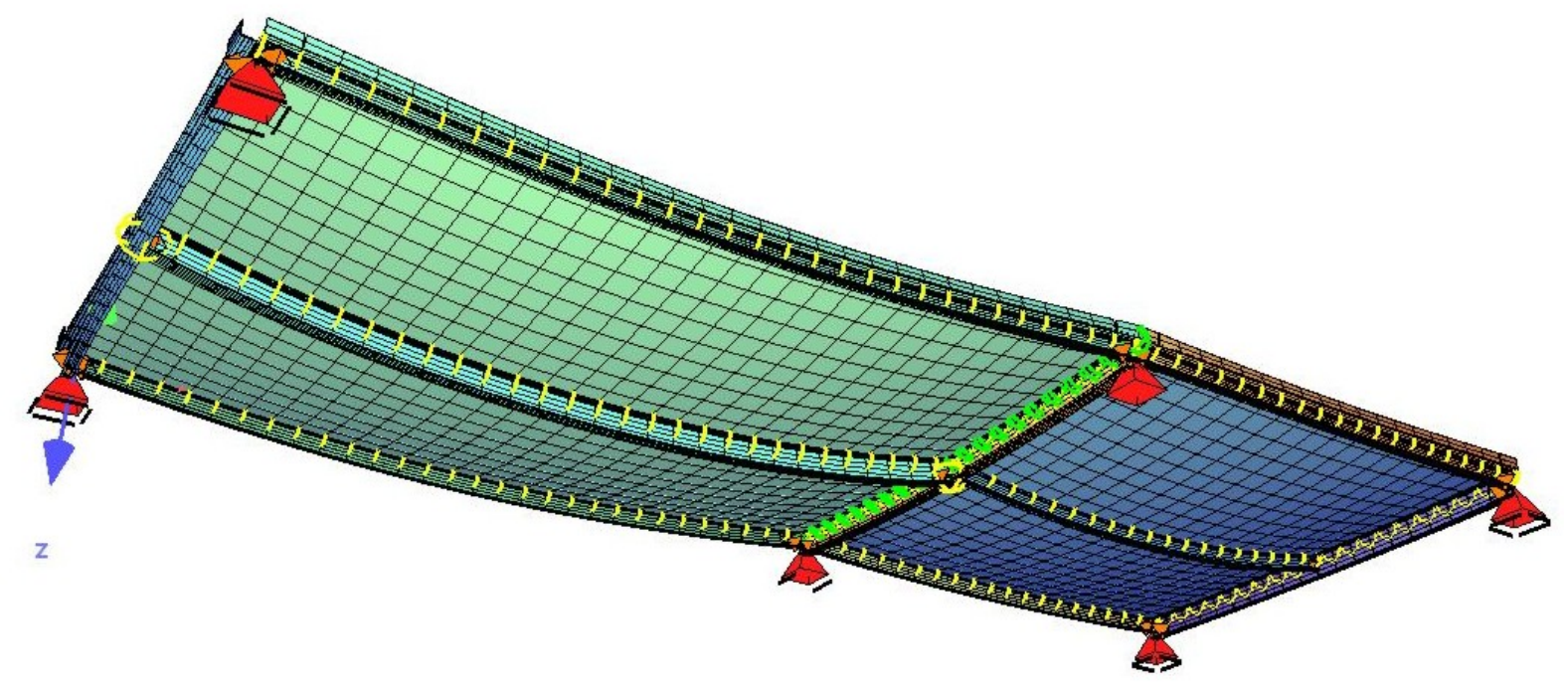

Fig. 7.1: Model of first munich test

The system is generally modelled according to Chapter 5 . The dimensions of the specimen are given in Section 3.1.1. The model is shown in Figure 7.1 and the input file can be found in Appendix B.6. The cross-sections of the edge beams include the angles L 120x80x8, which were used as formwork around the slab edges, since these provided considerable stiffness. A spring element is used at the connection of the unprotected secondary beams to the intermediate beam in order to take into account a certain stiffness of the joints. The assumption made in Section 5.3, that the connections can be modelled as perfectly hinged, is conservative for designing new structures but it is inappropriate for reproducing test results. The cracks above the intermediate beam can be modelled discretely since the actual location is known. Two rows of nodes are modelled where the cracks occurred and the elements are separated at this location. Only the displacements of the nodes are coupled but not the rotations. The yielding of the reinforcement is taken into account by spring elements between the double nodes with a plastic moment per node of:

$$
M_{p l}=a_{s} f_{y} d / n=1.88 \cdot 50.0 \cdot 0.08 / 4=1.9 \mathrm{kNm}
$$

Where $a_{s}$ is the amount of the top reinforcement, $f_{y}$ the yield strength, $d$ the internal lever arm in the hot cross-section and $n$ is the number of nodes per meter. The discrete modelling of the cracks is necessary for modelling the fire test since these have large effects on the stiffness and force distribution. If the method of this work is used for designing new structures, enough reinforcement will be assumed above the intermediate beams that this will not yield and no bending hinge will occur. In this case the slab can be assumed continuously without cracks above the intermediate beams which simplifies the modelling effort considerably.

The temperatures in the beams, measured in the top and bottom flanges and in the webs, are shown in Table 7.1. A mean temperature $\theta_{m}$ is calculated with Equation (4.37). The Young's modulus $E_{\theta}$ of each 
beam is reduced according to DIN EN 1994-1-2, Table 3.2 [32] and the thermal strain $\varepsilon_{\theta}$ is determined according to DIN EN 1994-1-2, 3.3.1 [32].

Table 7.1: Temperatures and material properties of the beams of Munich test 1 after 40 minutes

\begin{tabular}{|l|c|c|c|c|c|c|c|}
\hline Beam & Profile & $\theta_{f l, t o p}$ & $\theta_{\text {web }}$ & $\theta_{f l, b o t}$ & $\theta_{m}$ & $E_{\theta}$ & $\varepsilon_{\theta}$ \\
\hline & & {$\left[{ }^{\circ} \mathrm{C}\right]$} & {$\left[{ }^{\circ} \mathrm{C}\right]$} & {$\left[{ }^{\circ} \mathrm{C}\right]$} & {$\left[{ }^{\circ} \mathrm{C}\right]$} & {$[\mathrm{MPa}]$} & {$[\% o]$} \\
\hline Edge left & IPE240 & 243 & 316 & 503 & 352 & 157000 & 4.479 \\
\hline Intermediate & IPE240 & 307 & 369 & 590 & 419 & 143000 & 5.493 \\
\hline Edge right & IPE240 & 177 & 235 & 220 & 212 & 186500 & 2.481 \\
\hline Edge top left & IPE160 & 239 & 275 & 294 & 270 & 174000 & 3.285 \\
\hline Secondary left & IPE240 & 665 & 819 & 819 & 770 & 21400 & 11.00 \\
\hline Edge bottom left & IPE160 & 194 & 259 & 257 & 238 & 181000 & 2.842 \\
\hline Edge top right & IPE160 & 200 & 270 & 260 & 245 & 180000 & 2.938 \\
\hline Secondary right & IPE160 & 721 & 964 & 926 & 876 & 18900 & 11.00 \\
\hline Edge bottom right & IPE160 & 135 & 181 & 180 & 166 & 196000 & 1.865 \\
\hline
\end{tabular}

Since the temperature development in the furnace was slightly non-homogeneous, the temperatures in the slab were also not homogeneous. Two different temperature profiles are therefore used in the model as shown in Table 7.2. The location of the measuring points can be seen in Figure 3.5. The substitute thermal loadings $\varepsilon_{\theta, \text { subs }}$ and $\kappa_{\theta, \text { subs }}$ in Table 7.2 are determined analytically with Equation (4.21) and Equation (4.22).

Table 7.2: Temperatures in slab and substitute thermal load of Munich test 1 after 40 minutes

\begin{tabular}{|l|c|c|c|c|c|c|c|}
\hline Measuring point & $\mathrm{a}$ & $\mathrm{b}$ & $\mathrm{c}$ & $\mathrm{d}$ & $\mathrm{e}$ & $\varepsilon_{\theta \text {,subs }}$ & $\kappa_{\theta \text {,subs }}$ \\
\hline & {$\left[{ }^{\circ} \mathrm{C}\right]$} & {$\left[{ }^{\circ} \mathrm{C}\right]$} & {$\left[{ }^{\circ} \mathrm{C}\right]$} & {$\left[{ }^{\circ} \mathrm{C}\right]$} & {$\left[{ }^{\circ} \mathrm{C}\right]$} & {$[\%$ o } & {$[1 / \mathrm{km}]$} \\
\hline 11 larger panel & 700 & 302 & 171 & 60 & 38 & 1.161 & 44.20 \\
\hline 14 smaller panel & 722 & 302 & 100 & 74 & 54 & 1.101 & 41.84 \\
\hline
\end{tabular}

The stiffness of the slab at the ultimate limit state $E_{c m, \theta}^{I I}$ is determined as follows. The top reinforcement of $1.88 \mathrm{~cm}^{2} / \mathrm{m}$ in both directions has a distance $d_{1}$ from the top surface of the slab of $2.5 \mathrm{~cm}$. This leads to a height of the effective tension area of:

$$
h_{c t, e f f}=2.5 d_{1}=2.5 \cdot 2.5=6.25 \mathrm{~cm}>h / 2=6 \mathrm{~cm}<h=12 \mathrm{~cm}
$$

The reinforcement ratio $\rho_{c}$ and the effective reinforcement ratio $\rho_{c, e f f}$ can be calculated with Equation (5.19) and Equation (5.20).

$$
\begin{aligned}
& \rho_{c}=\frac{A_{s}}{A_{c}}=\frac{1.88}{100 \cdot 12}=0.001567 \\
& \rho_{c, e f f}=\frac{A_{s}}{A_{c t, e f f}}=\frac{1.88}{100 \cdot 6.25}=0.003008
\end{aligned}
$$

The cross-section centre of the larger slab panel reached a temperature of $\theta_{m}=171^{\circ} \mathrm{C}$ and the smaller panel $\theta_{m}=100^{\circ} \mathrm{C}$. The outcome of this is a reduction factor for cross-section softening of $k_{E, \theta, \text { mean }}=$ 0.488 for the larger panel and $k_{E, \theta, \text { mean }}=0.625$ for the smaller panel which can be found in Table 4.5 . With a measured concrete tensile strength of $f_{c t m}=2.72 \mathrm{~N} / \mathrm{mm}^{2}$ and a measured Young's modulus $E_{c m}=$ 
$32881 \mathrm{~N} / \mathrm{mm}^{2}$ as shown in Table 3.1, the stiffness of the larger slab panel at the ultimate limit state can be calculated with Equation (5.18) as:

$$
\begin{aligned}
E_{c m, \theta}^{I I} & =k_{E, \theta, \text { mean }} E_{c m}^{I I}=k_{E, \theta, \text { mean }} \frac{f_{y} \rho_{c}}{\frac{f_{y}}{E_{s}}-\beta_{t}\left[\frac{f_{c t, e f f}}{E_{s}}\left(\frac{1}{\rho_{c, e f f}}+\frac{E_{s}}{E_{c, e f f}}-1\right)-\frac{f_{c t, e f f}}{E_{c, e f f}}\right]} \\
& =0.488 \frac{500}{\frac{500 \cdot 0.001567}{200000}-0.25\left[\frac{2.72}{200000}\left(\frac{1}{0.003008}+\frac{200000}{32881}-1\right)-\frac{2.72}{32881}\right]}=278 \mathrm{~N} / \mathrm{mm}^{2}
\end{aligned}
$$

The stiffness of the smaller slab panel results in a slightly higher value of $E_{c m, \theta}^{I I}=357 \mathrm{~N} / \mathrm{mm}^{2}$ since the mean temperature remained lower.

The mechanical loading is applied to the model using a uniformly distributed load on the slab. The self weight of the structure and the load of the sand bags result in a total loading of $5.1 \mathrm{kN} / \mathrm{m}^{2}$. The vertical deformations obtained with the finite element model are compared in Figure 7.2 with the deformations measured in the test. The results are in good agreement within the limits of the uncertainties as described above. Only the deformations of the edge beams are smaller in the simulation. The reason is that the shear connection between the I-beams and the L-profiles is modelled rigidly. In fact the L-profiles were only welded at a few spots to the I-beams. The connection was therefore much softer in the test but this is difficult to take into account at the numerical model. Without the L-profiles the deformations of the edge beams are about $20 \%$ larger in the simulation compared to the test.
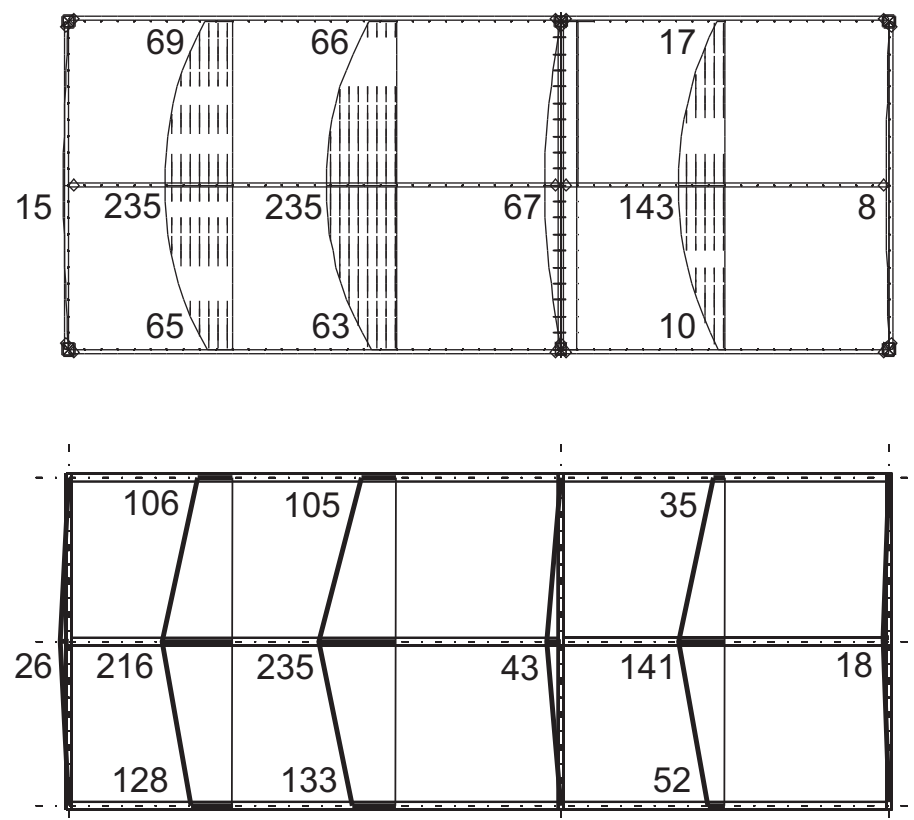

Fig. 7.2: Vertical deformations in $[\mathrm{mm}]$ after 40 minutes, FEA results (above) and test data (below) at Munich test 1

Figure 7.3 shows the principal stresses at the top surface of the slab. It can be seen that these are largely smaller than the tensile strength of the concrete which was measured with $f_{c t m}=2.72 \mathrm{~N} / \mathrm{mm}^{2}$. The tensile strength is only exceeded at the corners. The reason for that is the coarse element mesh and that the supports are modelled by fixing single nodes, which leads to singularities in the numerical model and to stress peaks. Since the tensile strength of the concrete is not reached, the reinforcement is unloaded and the mechanical load is transferred purely by tensile stresses in the concrete. Determining a required reinforcement amount with the design procedure described in Section 6.1 and comparing it 


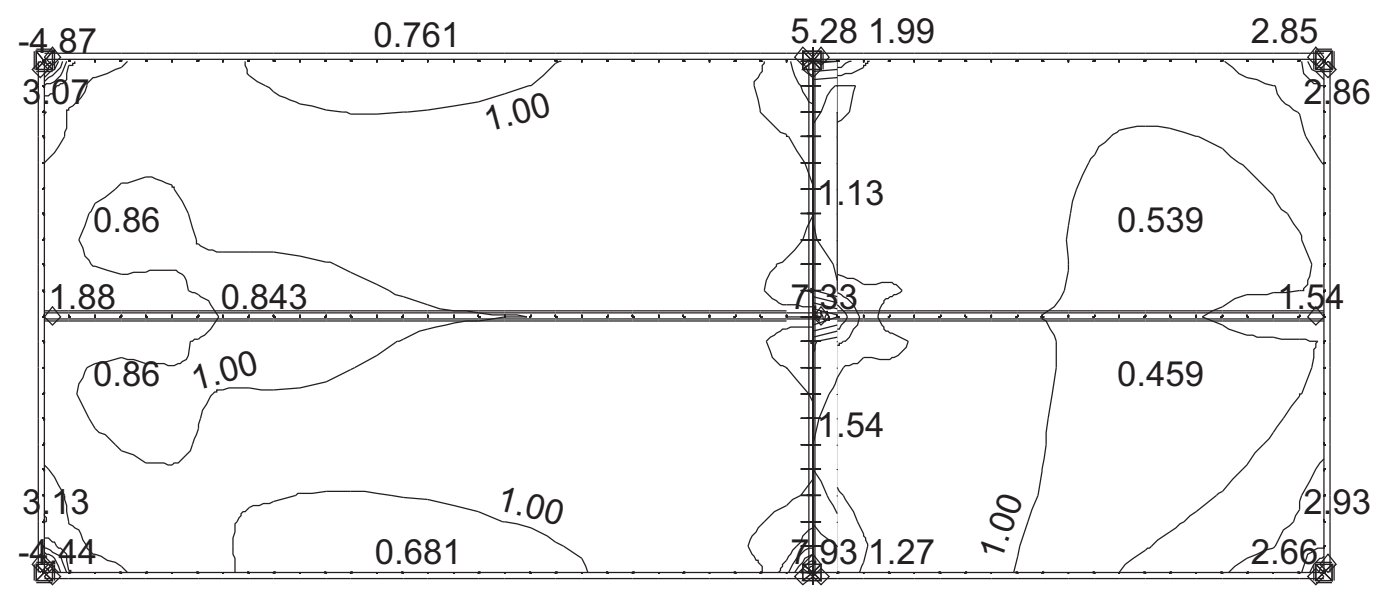

Fig. 7.3: Principal stresses I in $\left[\mathrm{N} / \mathrm{mm}^{2}\right]$ at top surface, Munich test 1

with the available reinforcement in the test is therefore not meaningful.

The design of the beams is illustrated on the most heavily loaded edge beam which is one of the secondary beams of the larger slab panel in longitudinal direction. Only the I-section is taken into account for calculating the bending resistance of the beam. The static system and cross-section is shown in Figure 7.4 .
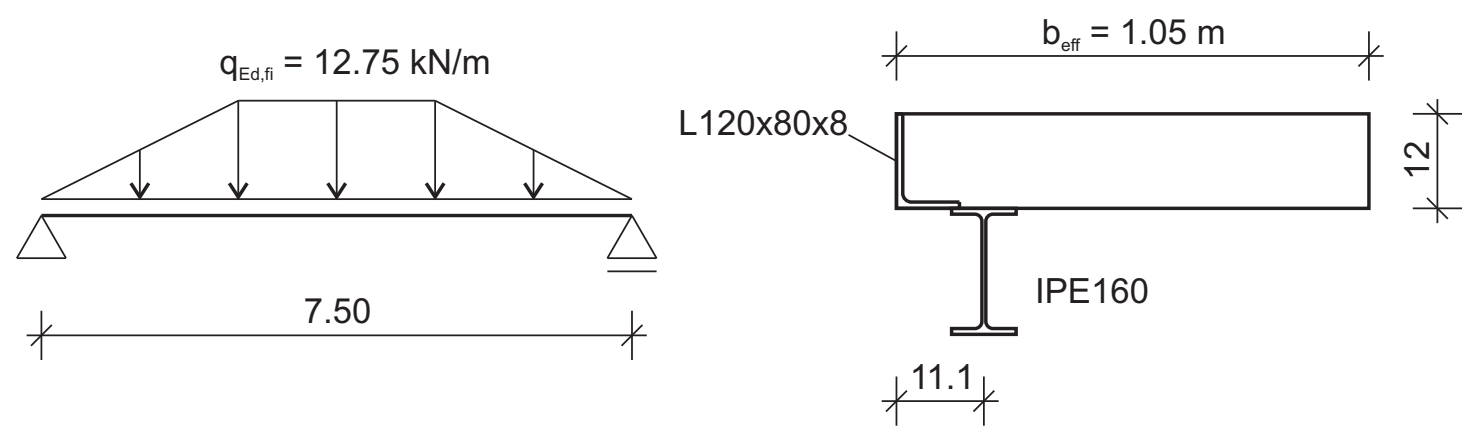

Fig. 7.4: System (left) and cross-section (right) of edge beam Munich test 1

The loading is assumed to be trapezoidal as described in Section 6.2:

$$
q_{E d, f i}=5.1 \mathrm{kN} / \mathrm{m}^{2} \cdot 2.5 \mathrm{~m}=12.75 \mathrm{kN} / \mathrm{m}
$$

This loading leads to a bending moment at midspan of $M_{E d, f i}=76.4 \mathrm{kNm}$. The effective width $b_{\text {eff }}$ is determined according to DIN EN 1994-1-1, 5.4.1.2 [31]:

$$
b_{e f f}=b_{0}+\frac{L_{e}}{8}=0.111+\frac{7.50}{8}=1.05 m
$$

The temperature of the steel beam remained lower than $300^{\circ} \mathrm{C}$ as shown in Figure 3.6 and the steel kept its full yield strength which was measured with $f_{y}=315 \mathrm{~N} / \mathrm{mm}^{2}$. This results in a plastic resistance of the steel section of:

$$
N_{p l, a, R d, f i}=A_{a} f_{y}=20.1 \cdot 31.5=633 k N
$$

With a measured compressive strength of the concrete of $f_{c m}=33.1 \mathrm{~N} / \mathrm{mm}^{2}$, the depth of the compres- 
sion zone can be calculated as follows:

$$
x_{p l}=\frac{N_{p l, a, R d, f i}}{f_{c m} b_{e f f}}=\frac{633}{3.31 \cdot 105}=1.8 \mathrm{~cm}
$$

This results in a bending resistance of:

$$
M_{f i, R d}^{+}=N_{p l, a, R d, f i}\left(\frac{h_{a}}{2}+h_{c}-\frac{x_{p l}}{2}\right)=633\left(\frac{0.16}{2}+0.12-\frac{0.018}{2}\right)=121 \mathrm{kNm}
$$

The bending resistance is larger than the bending moment at midspan in spite of the conservative assumptions in the design method.

It could be shown that the behaviour of the first Munich test can be simulated with the proposed method of this work. The most remarkable result is that the stresses at the slab centres did not reach the tensile strength. No cracks occurred within the slab panels and the loads were transferred purely through tensile stresses in the concrete. If the fire had lasted longer, the tensile strength of the concrete could possibly have been exceeded and large cracks at the slab centre or even collapse of the whole structure could have occurred.

\subsection{Second Munich fire test}

The slab in the second Munich test did also not collapse. The time 40 minutes after test start will be considered as for the first test. The temperatures in the furnace, in the unprotected secondary beams and in the steel sheeting reached their maximum at this time as shown in Figure 3.12 to Figure 3.14. The slab was therefore most heavily loaded after 40 minutes. The dimensions of the specimen are given in Section 3.1.1. The finite element model is shown in Figure 7.5 and the input file can be found in

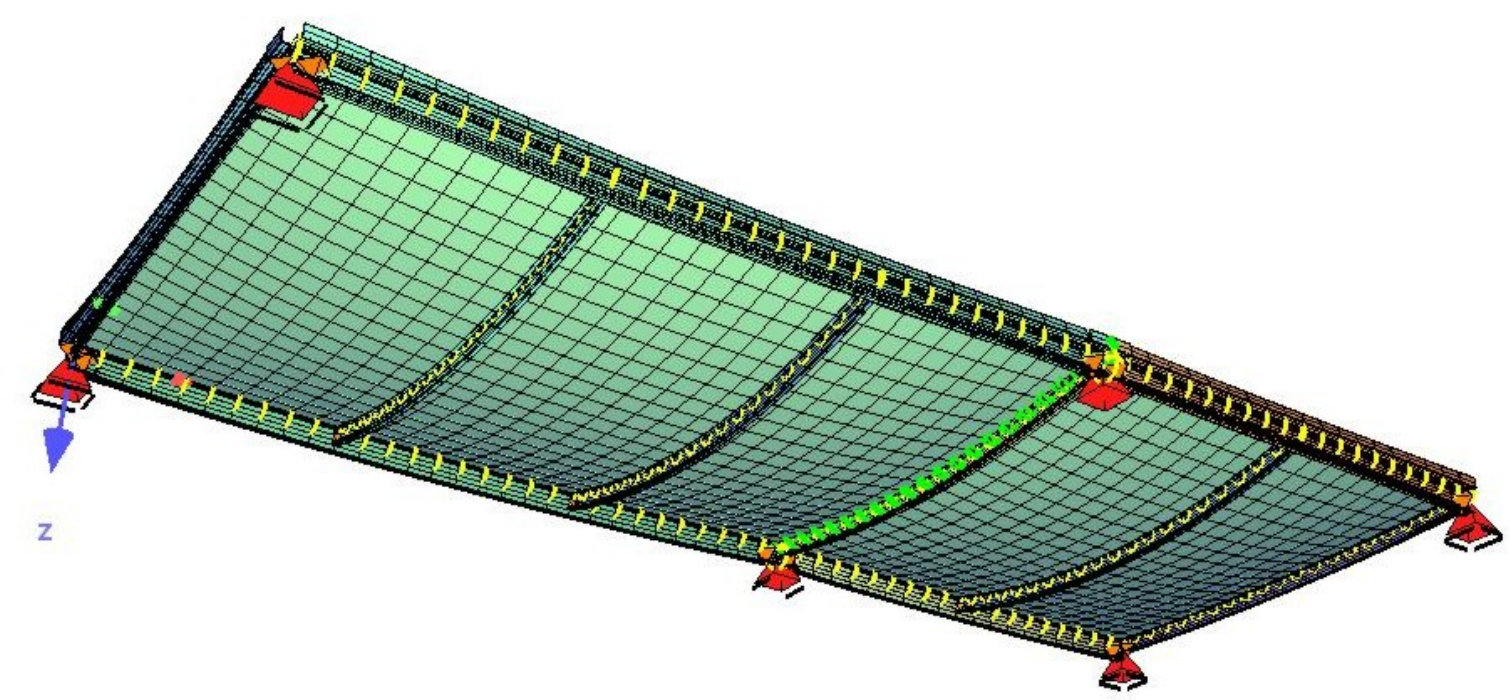

Fig. 7.5: Model of second munich test

Annex B.7. The slab is modelled with an effective thickness of $h_{e f f}=11.2 \mathrm{~cm}$ in both directions as described in Section 5.2. The angles L 120x80x8, that were used as formwork, are taken into account at the edge beams. The crack above the intermediate beam is modelled with two rows of nodes whose displacements are coupled. The rotations are coupled by the use of springs with a plastic moment per 
node of:

$$
M_{p l}=a_{s} f_{y} d / n=1.88 \cdot 50.0 \cdot 0.07 / 4=1.6 \mathrm{kNm}
$$

The temperatures in the flanges and the webs of the steel beams, that were measured after 40 minutes, are assembled in Table 7.3. With the average temperature $\theta_{m}$, the reduced Young's modulus $E_{\theta}$ is calculated according to DIN EN 1994-1-2, Table 3.2 [32] and the thermal strain $\varepsilon_{\theta}$ is determined according to DIN EN 1994-1-2, 3.3.1 [32]. These values are also shown in Table 7.3 for every beam of the model.

Table 7.3: Temperatures and material properties of the beams of Munich test 2 after 40 minutes

\begin{tabular}{|l|c|c|c|c|c|c|c|}
\hline Beam & Profile & $\theta_{f l, t o p}$ & $\theta_{\text {web }}$ & $\theta_{f l, b o t}$ & $\theta_{m}$ & $E_{\theta}$ & $\varepsilon_{\theta}$ \\
\hline & & {$\left[{ }^{\circ} \mathrm{C}\right]$} & {$\left[{ }^{\circ} \mathrm{C}\right]$} & {$\left[{ }^{\circ} \mathrm{C}\right]$} & {$\left[{ }^{\circ} \mathrm{C}\right]$} & {$[\mathrm{MPa}]$} & {$[\%$ o $]$} \\
\hline Edge left & IPE160 & 244 & 284 & 306 & 278 & 172550 & 3.408 \\
\hline Intermediate & IPE160 & 311 & 317 & 389 & 338 & 168000 & 4.265 \\
\hline Edge right & IPE160 & 233 & 270 & 291 & 265 & 175381 & 3.217 \\
\hline Edge top left & IPE240 & 176 & 234 & 257 & 223 & 184175 & 2.633 \\
\hline Unpr. 2nd very left & IPE160 & 670 & 809 & 809 & 766 & 21797 & 11.00 \\
\hline Edge bottom left & IPE240 & 192 & 255 & 280 & 243 & 180000 & 2.908 \\
\hline Edge top right & IPE240 & 234 & 311 & 342 & 296 & 168743 & 3.667 \\
\hline Unpr. 2nd right & IPE160 & 684 & 826 & 826 & 782 & 20453 & 11.00 \\
\hline Edge bottom right & IPE240 & 179 & 237 & 261 & 226 & 183500 & 2.677 \\
\hline Unpr. 2nd left & IPE160 & 683 & 825 & 825 & 780 & 20549 & 11.00 \\
\hline
\end{tabular}

The substitute thermal loading on the slab is determined by finite element models with the method described in Section 4.2.3. The thermal analysis is performed as explained in Section 4.1.2 using the average gas temperature in the furnace for the temperature-time curve and the view factors as shown in Table 4.2. The resulting substitute thermal strain and curvature in the longitudinal and transversal direction are shown in Table 7.4.

Table 7.4: Substitute thermal loading on slab of Munich test 2 after 40 minutes

\begin{tabular}{|l|c|c|}
\hline & $\varepsilon_{\theta, \text { subs }}[\%$ o $]$ & $\kappa_{\theta, \text { subs }}[1 / \mathrm{km}]$ \\
\hline Longitudinal & 1.045 & 43.0 \\
\hline Transversal & 0.655 & 29.6 \\
\hline
\end{tabular}

The effective tension area has the same height as in test 1 since the reinforcement mesh was placed at the same distance $d_{1}$ from the top surface of the slab.

$$
h_{c t, e f f}=2.5 d_{1}=2.5 \cdot 2.5=6.25 \mathrm{~cm}>h_{\text {eff }} / 2=5.6 \mathrm{~cm}<h_{\text {eff }}=11.2 \mathrm{~cm}
$$

With an available reinforcement area of $1.88 \mathrm{~cm}^{2} / \mathrm{m}$ in both directions, the reinforcement ratios become:

$$
\begin{aligned}
& \rho_{c}=\frac{A_{s}}{A_{c}}=\frac{1.88}{100 \cdot 11.2}=0.001679 \\
& \rho_{c, e f f}=\frac{A_{s}}{A_{c t, e f f}}=\frac{1.88}{100 \cdot 6.25}=0.003008
\end{aligned}
$$

The temperature at the centre of the slab cross-section is determined with the finite element model as 
$\theta_{m}=128^{\circ} \mathrm{C}$. This leads to a reduction factor for cross-section softening of $k_{E, \theta, \text { mean }}=0.571$ which can be found in Table 4.5. The actual tensile strength of the concrete was measured to $f_{c t m}=3.59 \mathrm{~N} / \mathrm{mm}^{2}$ and the Young's modulus to $E_{c m}=36845 \mathrm{~N} / \mathrm{mm}^{2}$ as shown in Table 3.1. The stiffness at the ultimate limit state can then be calculated as:

$$
\begin{aligned}
E_{c m, \theta}^{I I} & =k_{E, \theta, \text { mean }} E_{c m}^{I I}=k_{E, \theta, \text { mean }} \frac{f_{y} \rho_{c}}{\frac{f_{y}}{E_{s}}-\beta_{t}\left[\frac{f_{c t, e f f}}{E_{s}}\left(\frac{1}{\rho_{c, e f f}}+\frac{E_{s}}{E_{c, e f f}}-1\right)-\frac{f_{c t, e f f}}{E_{c, e f f}}\right]} \\
& =0.571 \frac{500 \cdot 0.001679}{\frac{500}{200000}-0.25\left[\frac{3.59}{200000}\left(\frac{1}{0.003008}+\frac{200000}{36845}-1\right)-\frac{3.59}{36845}\right]}=473 \mathrm{~N} / \mathrm{mm}^{2}
\end{aligned}
$$

The total mechanical loading is again $5.1 \mathrm{kN} / \mathrm{m}^{2}$ as in test 1 and is applied as a distributed load on the slab. The vertical deformations obtained with the finite element model are compared in Figure 7.6 with the deformations measured in the test. The results are in good agreement. Like in the first test, the deformations of the edge beams are simulated smaller due to the rigid connection of the L-profiles.
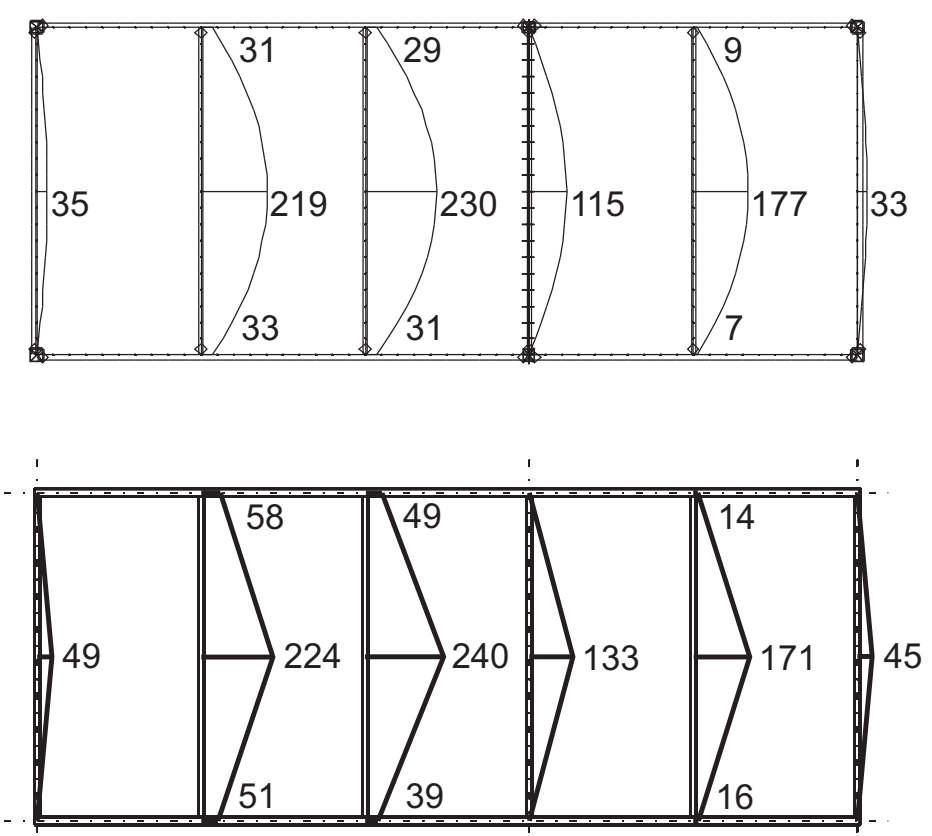

Fig. 7.6: Vertical deformations in $[\mathrm{mm}]$ after 40 minutes, FEA results (above) and test data (below) at Munich test 2

The stresses at the top surface of the slab are again largely smaller than the tensile strength of the concrete like in test 1. This can be seen in Figure 7.7. For this reason no cracks occurred at the centre of the slab panels. This shows that the loads were transferred purely by tensile stresses in the concrete like in test 1 . The design procedure of the beams is similar to test 1 . Since it provides no new information, the design will not be shown here.

To summarise, the simulation results of the second Munich fire test are in good agreement with the test data. This shows that the proposed method of this work includes realistic assumptions and can be used safely for the design of composite slab systems in fire. 


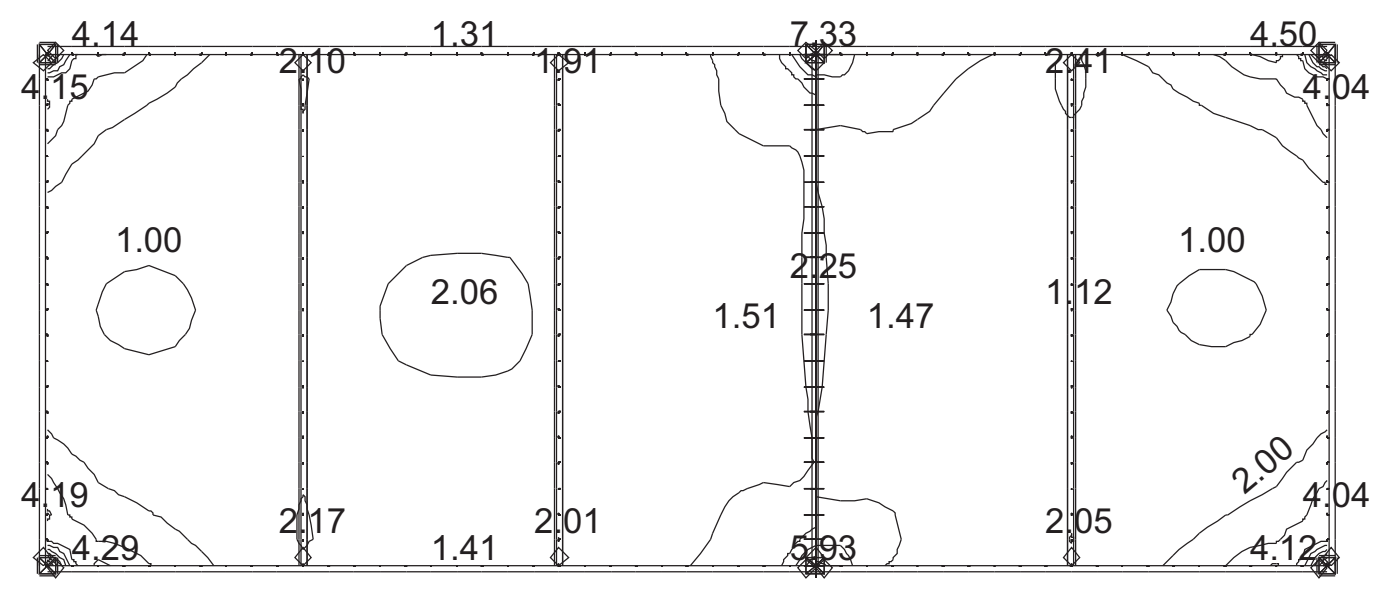

Fig. 7.7: Principal stresses I in $\left[\mathrm{N} / \mathrm{mm}^{2}\right]$ at top surface, Munich test 2

\subsection{FRACOF test}

The time considered in the FRACOF test is 105 minutes after test start. The ultimate limit state was reached as a large crack occurred at this time at the slab centre in the transversal direction. The experiment was stopped shortly after this incident. The dimensions of the specimen are given in Section 3.2.1. The numerical model is shown in Figure 7.8 and the input file can be found in Annex B.8. The slab is modelled with an effective thickness of $h_{e f f}=12.0 \mathrm{~cm}$ in both directions as described in Section 5.2. The

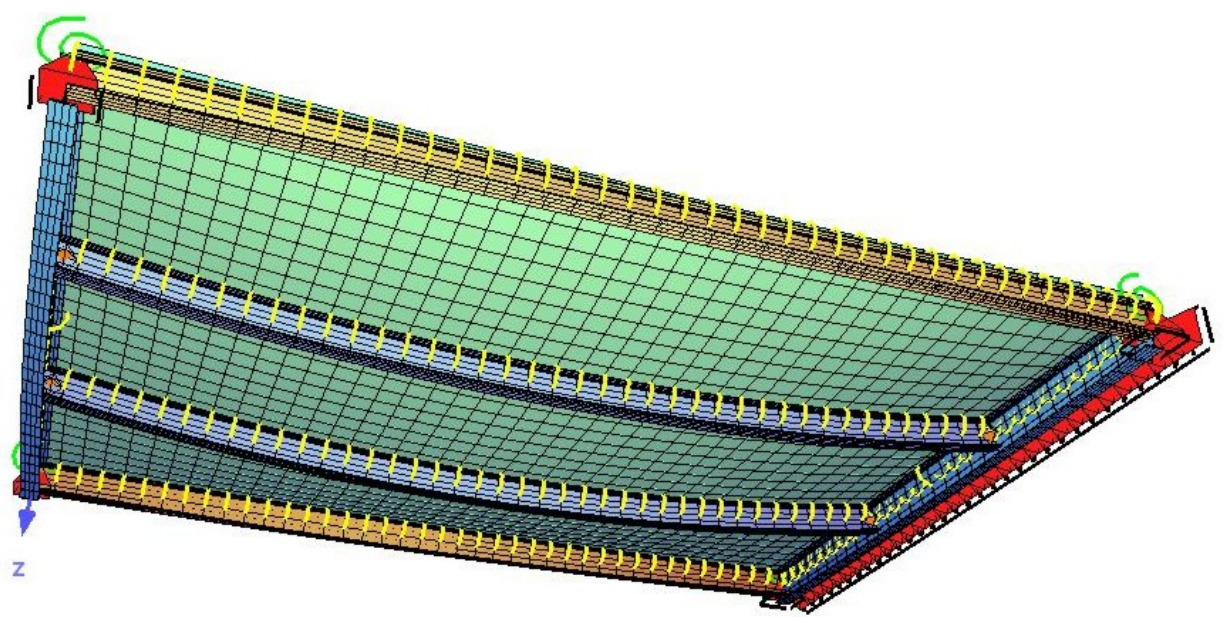

Fig. 7.8: Model of FRACOF test

steel beam at one shorter edge, which was attached in order to simulate continuity, is taken into account in the model by horizontal supports along this edge. The steel beam for continuity at the longer edge is neglected in the model since it had no effect in the test due to buckling of the uncovered reinforcement. A small rotational restraint at the connections between the edge beams and the columns is taken into account with rotational spring elements.

The mechanical loading consists of a self-weight of $g=3.254 \mathrm{kN} / \mathrm{m}^{2}$ and an additional load from the sand bags of $q=3.87 \mathrm{kN} / \mathrm{m}^{2}$. The resulting load of $7.124 \mathrm{kN} / \mathrm{m}^{2}$ is applied to the model as a distributed load. The unprotected beams reached a temperature of about $1000^{\circ} \mathrm{C}$ which leads to a reduced Young's modulus of $E_{\theta}=9450 \mathrm{~N} / \mathrm{mm}^{2}$ according to DIN EN 1994-1-2, Table 3.2 [32] and a thermal strain of $\varepsilon_{\theta}=13.8 \%$ according to DIN EN 1994-1-2, 3.3.1 [32]. The temperatures of the protected edge beams 
are shown in Figure 3.21. After 105 minutes the top flange reached about $\theta_{f l, t o p}=115^{\circ} \mathrm{C}$, the web $\theta_{w e b}=160^{\circ} \mathrm{C}$ and the bottom flange $\theta_{f l, b o t}=240^{\circ} \mathrm{C}$. The $F R A C O F$ project report [49] does not describe whether these temperatures were measured in the primary or secondary edge beams. For this reason, the temperatures are assumed similarly in all edge beams. The average temperature can be calculated as $\theta_{m}=170^{\circ} \mathrm{C}$ which leads to a reduced Young's modulus of $E_{\theta}=195300 \mathrm{~N} / \mathrm{mm}^{2}$ and a thermal strain of $\varepsilon_{\theta}=1.914 \%$.

The substitute thermal loading on the slab is determined by finite element models with the method described in Section 4.2.3. The thermal analysis is performed as explained in Section 4.1.2 using the standard fire curve for the temperature development of the gas in the furnace and the view factors as shown in Table 4.2. The resulting substitute thermal strain and curvature in the longitudinal and transversal direction are shown in Table 7.5. The directions in Table 7.5 refer to the direction of the steel sheeting, not to the direction of the slab panel.

Table 7.5: Substitute thermal loading on slab of FRACOF test after 105 minutes

\begin{tabular}{|l|c|c|}
\hline Direction of sheeting & $\varepsilon_{\theta, \text { subs }}[\% o]$ & $\kappa_{\theta, \text { subs }}[1 / \mathrm{km}]$ \\
\hline Longitudinal & 1.20 & 77.3 \\
\hline Transversal & 2.11 & 73.0 \\
\hline
\end{tabular}

A top reinforcement with an area of $2.57 \mathrm{~cm}^{2} / \mathrm{m}$ in both directions was placed in the specimen with a distance from the top surface of the slab of $d_{1}=5.0 \mathrm{~cm}$. The effective tension area is calculated as follows:

$$
\begin{aligned}
& h_{c t, e f f}=2.5 d_{1} \geq h_{e f f} / 2 \leq h_{e f f} \\
& h_{c t, e f f}=h_{e f f}=12 \mathrm{~cm}
\end{aligned}
$$

The reinforcement ratio $\rho_{c}$ and the effective reinforcement ratio $\rho_{c, \text { eff }}$ offer the same value in this case since the effective tension area covers the entire cross-section.

$$
\begin{aligned}
& \rho_{c}=\frac{A_{s}}{A_{c}}=\frac{2.57}{100 \cdot 12}=0.002142 \\
& \rho_{c, \text { eff }}=\rho_{c}=0.002142
\end{aligned}
$$

The temperature at the centre of the slab cross-section is determined with the finite element model that was used for determining the substitute thermal loading of the slab. With $\theta_{m}=340^{\circ} \mathrm{C}$ the reduction factor for cross-section softening becomes $k_{E, \theta, \text { mean }}=0.258$ which can be found in Table 4.5. The compressive strength of the concrete was measured as $f_{c m}=36.7 \mathrm{~N} / \mathrm{mm}^{2}$. The tensile strength and the Young's modulus were not measured. These will be interpolated in Table 3.1 of DIN EN 1992-1-1 [26] to $f_{c t m}=2.82 \mathrm{~N} / \mathrm{mm}^{2}$ and $E_{c m}=32480 \mathrm{~N} / \mathrm{mm}^{2}$. The stiffness at the ultimate limit state can then be calculated as:

$$
\begin{aligned}
E_{c m, \theta}^{I I} & =k_{E, \theta, \text { mean }} E_{c m}^{I I}=k_{E, \theta, \text { mean }} \frac{f_{y} \rho_{c}}{\frac{f_{y}}{E_{s}}-\beta_{t}\left[\frac{f_{c t, e f f}}{E_{s}}\left(\frac{1}{\rho_{c, e f f}}+\frac{E_{s}}{E_{c, e f f}}-1\right)-\frac{f_{c t, e f f}}{E_{c, e f f}}\right]} \\
& =0.258 \frac{500 \cdot 0.002142}{\frac{500}{200000}-0.25\left[\frac{2.82}{200000}\left(\frac{1}{0.002142}+\frac{200000}{32480}-1\right)-\frac{2.82}{32480}\right]}=322 \mathrm{~N} / \mathrm{mm}^{2}
\end{aligned}
$$


A comparison between the vertical deformations is shown in Figure 7.9. The values of the test data are read from Figure 3.22. Two values are given for the beams since two curves are available in Figure 3.22 and it is not clear exactly which curve belongs to which beam. The simulation results are shown in brackets. Only one value is obtained for every beam since the model is symmetric. It can be seen that the results are in good agreement.

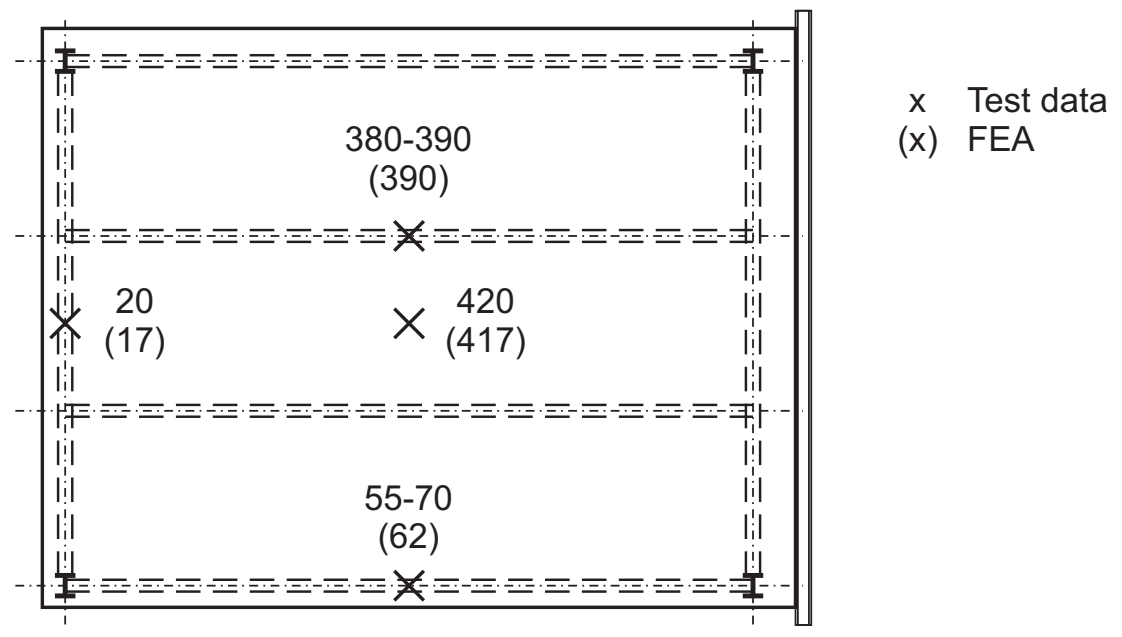

Fig. 7.9: Comparison of vertical deformations in $[\mathrm{mm}]$ at FRACOF test after 105 minutes

At the considered time of 105 minutes after test start a large crack occurred at the slab centre and the reinforcement in longitudinal direction ruptured in the crack. This can be explained as follows. A membrane force in longitudinal direction of $n_{x}=142 \mathrm{kN} / \mathrm{m}$ and a corresponding bending moment of $m_{x x}=-1.52 \mathrm{kNm} / \mathrm{m}$ at the centre of the slab can be determined with the numerical model. The design procedure with the effective thickness method, which was explained in Section 6.1.2, is applied on a cross-section as shown in Figure 7.10. The depth of the damaged zone in transversal direction is interpolated for a time of 105 minutes on the standard fire curve to $a_{z, \text { trans }}=17 \mathrm{~mm}$. The remaining rectangular cross-section above the ribs offers a depth of $80 \mathrm{~mm}$.

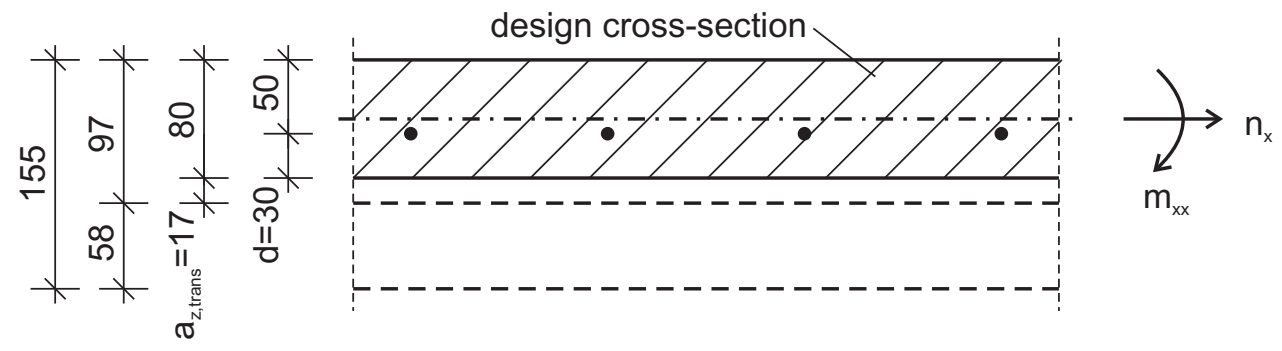

Fig. 7.10: Cross-section for design at slab centre

The internal forces cause a tensile stress at the top surface of the slab of:

$$
\sigma_{\text {top }}=\frac{n_{x}}{A_{c}}+\frac{m_{x x}}{W}=\frac{142}{100 \cdot 8.0}+\frac{152}{\frac{100 \cdot 8.0^{2}}{6}}=0.32 \mathrm{kN} / \mathrm{cm}^{2} \hat{=} 3.2 \mathrm{~N} / \mathrm{mm}^{2}
$$

This stress is larger than the average tensile strength of the concrete of $f_{c t m}=2.82 \mathrm{~N} / \mathrm{mm}^{2}$ and a first crack occurred. The entire load was transferred to the reinforcement which could not bear it as explained in the following.

The required reinforcement amount will be designed here with the method of non-dimensional moment 
parameters according to [55]. It should be noticed that both the compressive zone and the reinforcement lie below the neutral axis since the bending moment causes tension at the top surface of the slab and the reinforcement was placed at a relatively large distance from the top surface. The bending moment about the axis of the tensioned reinforcement is:

$$
m_{E d, s}=m_{E d}-n_{E d} z_{s 1}=-1.52-142(0.050-0.080 / 2)=-2.94 \mathrm{kNm} / \mathrm{m}
$$

The non-dimensional moment parameter can then be calculated as:

$$
\mu_{E d, s}=\frac{m_{E d, s}}{b d^{2} f_{c d}}=\frac{0.00294}{1.0 \cdot 0.030^{2} \cdot 36.7}=0.089
$$

The value for the compressive strength, that was measured in the test, is used and the safety factor is set to 1.0 for the fire case. A mechanical reinforcement ratio of $\omega=0.0935$ can be obtained with Table 6.3 of [55]. The required reinforcement amount with an actual yield strength of $f_{y}=594 \mathrm{~N} / \mathrm{mm}^{2}$ is then calculated as:

$$
a_{s, r e q}=\frac{1}{f_{y}}\left(\omega b d f_{c d}+n_{E d}\right)=\frac{1}{59.4}(0.0935 \cdot 100 \cdot 3.0 \cdot 3.67+142)=4.12 \mathrm{~cm}^{2} / \mathrm{m}
$$

The required amount is larger than the available amount of $a_{s, \text { available }}=2.57 \mathrm{~cm}^{2} / \mathrm{m}$. This shows that the available reinforcement could not bear the forces in the slab as soon as the crack occurred. As a result it ruptured and the crack could open unhindered.

To summarise, the results of the fire tests can be simulated in good agreement with the proposed method of this work. The method is thus validated and can be safely used for designing concrete and composite slab systems in case of fire. 


\section{Worked example}

In this chapter the way in which the proposed method can be applied for designing new structures will be summarised and illustrated. For this purpose, the worked example from the report of the DASt project [42] will be calculated with the method of this work. Figure 8.1 shows the plan view and cross-section of the considered slab system. The slab is part of a small office building. The cross-sections were dimensioned for ambient temperature which is not shown here. The structure shall fulfil a fire resistance class of R90.

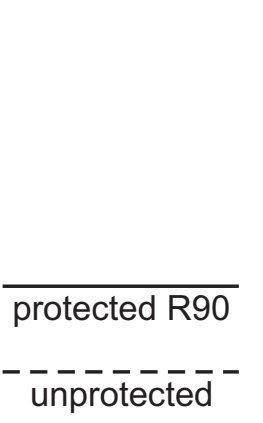

(ற)
(1)

(2)

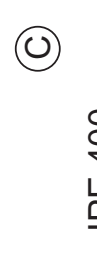

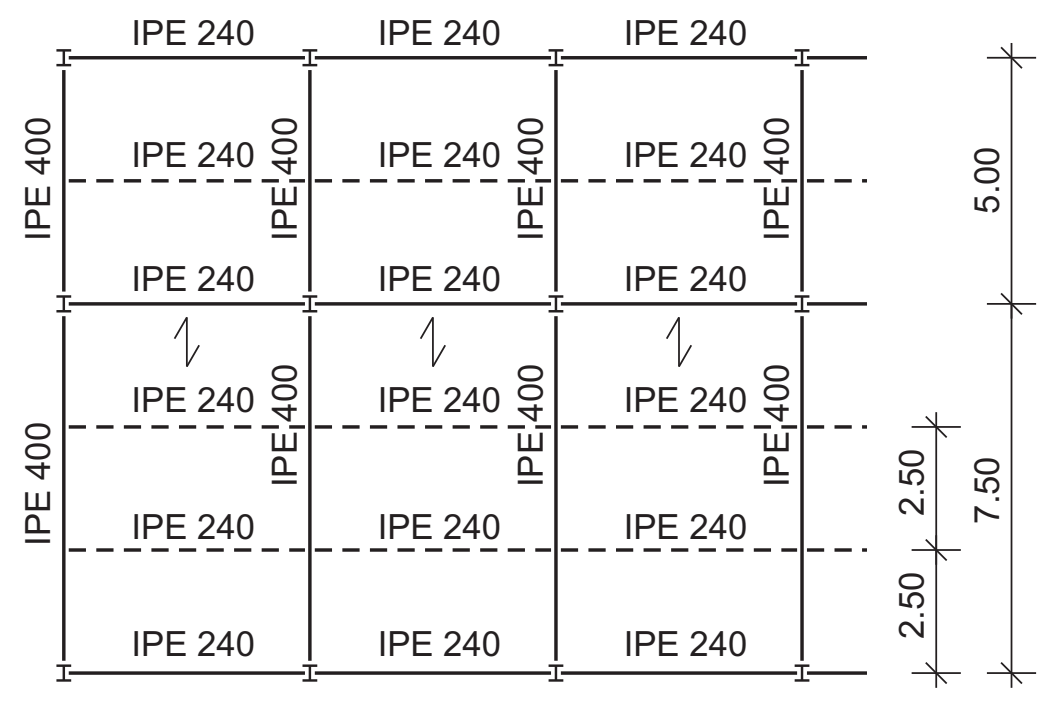
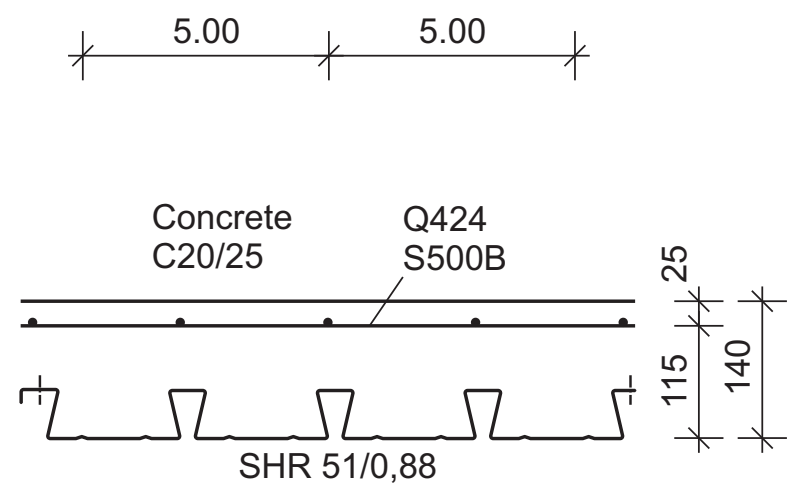

Fig. 8.1: Plan view (above) and cross-section (below) of worked example on the base of [42]

The slab system consists of a grid of primary beams IPE 400 and secondary beams IPE 240 in steel grade S235. It is supported by steel columns and horizontally braced. Both the columns and the bracing are protected against the fire and will be not further considered. The slab can be divided into several panels with a column at every corner. The larger panels have dimensions of $5.0 \mathrm{~m}$ by $7.5 \mathrm{~m}$ and the smaller panels of $5.0 \mathrm{~m}$ by $5.0 \mathrm{~m}$. A composite slab system type Superholorib SHR51 is used with a 
thickness of the steel sheeting of $0.88 \mathrm{~mm}$. It is finished to a total depth of $140 \mathrm{~mm}$ with concrete class C20/25. A top reinforcement, steel grade S500, is placed at a distance of $25 \mathrm{~mm}$ from the top surface of the slab. The required amount of this reinforcement is determined by the method of this work. The edge beams around the perimeter of each slab panel, that are shown in Figure 8.1 with bold lines, are protected against the fire. The protection system ensures that the maximum temperature in the beams does not exceed $540^{\circ} \mathrm{C}$. The secondary beams within the slab panels are left unprotected. These can be seen in Figure 8.1 on the dashed lines. The mechanical loading on the slab consists of following parts:

$$
\begin{array}{ll}
\text { self-weight } & g_{k}=3.5 \mathrm{kN} / \mathrm{m}^{2} \\
\text { additional dead load } & g_{k}=2.0 \mathrm{kN} / \mathrm{m}^{2} \\
\text { live load category B1 } & q_{k}=2.0 \mathrm{kN} / \mathrm{m}^{2} \\
\text { movable partitions } & q_{k}=0.8 \mathrm{kN} / \mathrm{m}^{2}
\end{array}
$$

In case of fire, these loads are combined for the accidental design situation. The quasi-permanent value $\psi_{2}$ is used for the combination factor as it is proposed in DIN EN 1991-1-2, 4.3.1 [24] and specified in the German national annex [25].

$$
q_{E d, f i}=g_{k}+\psi_{2} q_{k}=3.5+2.0+0.3(2.0+0.8)=6.34 k N / m^{2}
$$

\subsection{Modelling and results}

The structure is modelled according to Chapter 5. The static system is shown in Figure 8.2 and the input file can be found in Annex B.9. Only a part of the structure will be modelled since the single sections of the slab are similar. Two adjacent slab sections are considered in order to take into account the effects due to continuity. The composite slab is modelled with shell elements with a size of $0.25 \mathrm{~m}$ in both

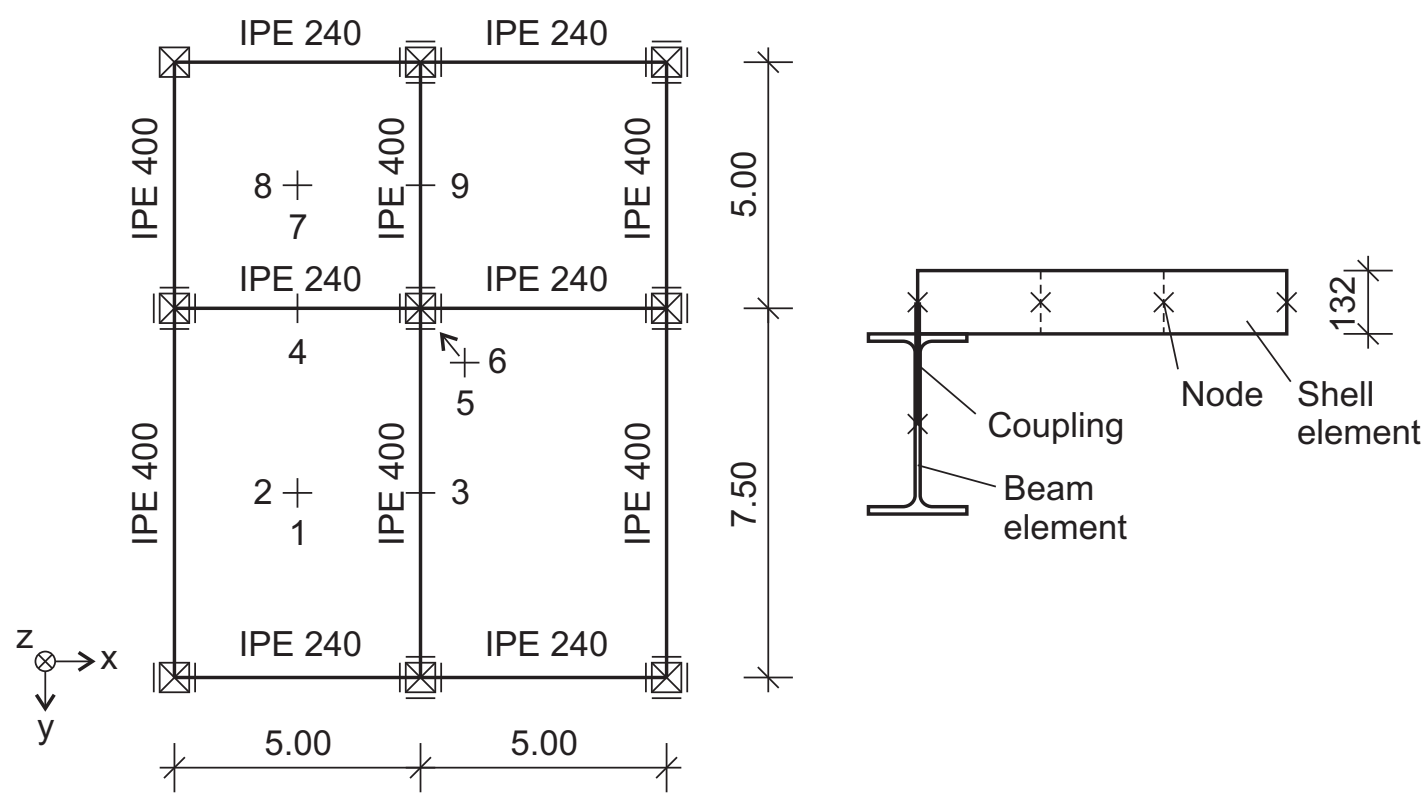

Fig. 8.2: Static system and considered points for design

directions and an effective thickness according to DIN EN 1994-1-2, Annex D.4 [32]:

$$
h_{2} / h_{1}=51 / 89=0.57<1.5
$$




$$
\begin{aligned}
& h_{1}=89 \mathrm{~mm}>40 \mathrm{~mm} \\
& h_{e f f}=h_{1}+0.5 h_{2}\left(\frac{l_{1}+l_{2}}{l_{1}+l_{3}}\right)=89+0.5 \cdot 51\left(\frac{114+138}{114+36}\right)=132 \mathrm{~mm}
\end{aligned}
$$

The protected steel beams are modelled with beam elements with an additional row of nodes that are coupled rigidly to the nodes of the slab. A bending hinge in all directions is included at both ends of each beam. The unprotected beams are neglected since these only have a minor effect but could be included if necessary. The columns are replaced by rigid supports in the vertical direction. In the horizontal direction, the system is simply supported.

The stiffness of the slab is determined according to Section 5.2.4. The reduction factor for crosssection softening is interpolated in Table 5.1 to $k_{E, \theta \text {,mean }}=0.360$. The top reinforcement is placed at a distance from the top surface of the slab of $d_{1}=2.5 \mathrm{~cm}$. The effective tension area can then be calculated as:

$$
\begin{aligned}
& h_{c t, e f f}=2.5 d_{1} \geq h_{e f f} / 2 \leq h_{e f f} \\
& h_{c t, e f f}=h_{e f f} / 2=6.6 \mathrm{~cm}
\end{aligned}
$$

The concrete class C20/25 provides an average Young's modulus of $E_{c m}=30000 \mathrm{~N} / \mathrm{mm}^{2}$ and tensile strength of $f_{c t m}=2.2 \mathrm{~N} / \mathrm{mm}^{2}$, both according to DIN EN 1992-1-1, Table 3.1 [26]. Both values are smaller than the minimum values that should be used for lower concrete classes that often have a higher strength in reality than required. The effective Young's modulus and tensile strength are therefore assumed as:

$$
\begin{aligned}
E_{c, e f f} & =33000 \mathrm{~N} / \mathrm{mm}^{2} \\
f_{c t, e f f} & =3.0 \mathrm{~N} / \mathrm{mm}^{2}
\end{aligned}
$$

To ensure that a distributed crack pattern can develop and to avoid only a single crack occurring, reinforcement rupture in this single crack and slab failure in terms of integrity, a minimum reinforcement should be used. The required amount can be calculated with Equation (5.25):

$$
A_{s, \min }=\frac{A_{c t, e f f}}{\frac{f_{y}}{f_{c t, e f f}}-\frac{E_{s}}{E_{c, e f f}}+1}=\frac{100 \cdot 6.6}{\frac{500}{3.0}-\frac{200000}{33000}+1}=4.08 \mathrm{~cm}^{2} / \mathrm{m}
$$

A reinforcement mesh Q424 is chosen that provides a steel area of $A_{s}=4.24 \mathrm{~cm}^{2} / \mathrm{m}$ in both directions. With this value, the reinforcement ratio $\rho_{c}$ and the effective reinforcement ratio $\rho_{c, e f f}$ can be calculated using Equation (5.19) and Equation (5.20):

$$
\begin{aligned}
& \rho_{c}=\frac{A_{s}}{A_{c}}=\frac{4.24}{100 \cdot 13.2}=0.003212 \\
& \rho_{c, e f f}=\frac{A_{s}}{A_{c t, e f f}}=\frac{4.24}{100 \cdot 6.6}=0.006424
\end{aligned}
$$

The stiffness at the ultimate limit state can then be determined with Equation (5.18) as:

$$
E_{c m, \theta}^{I I}=k_{E, \theta, \text { mean }} E_{c m}^{I I}=k_{E, \theta, \text { mean }} \frac{f_{y} \rho_{c}}{\frac{f_{y}}{E_{s}}-\beta_{t}\left[\frac{f_{c t, e f f}}{E_{s}}\left(\frac{1}{\rho_{c, e f f}}+\frac{E_{s}}{E_{c, e f f}}-1\right)-\frac{f_{c t, e f f}}{E_{c, e f f}}\right]}
$$




$$
=0.360 \frac{500 \cdot 0.003212}{\frac{500}{200000}-0.25\left[\frac{3.0}{200000}\left(\frac{1}{0.006424}+\frac{200000}{33000}-1\right)-\frac{3.0}{33000}\right]}=301 \mathrm{~N} / \mathrm{mm}^{2}
$$

This value is used for the Young's modulus of the slab in the finite element model. Two limiting values are considered for the stiffness of the steel beams. In load case 1, the beams reach their maximum temperature of $540^{\circ} \mathrm{C}$. This leads to a Young's modulus of $E_{\theta}=101640 \mathrm{~N} / \mathrm{mm}^{2}$ according to DIN EN 1994$1-2$, Table 3.2 [32] and a substitute thermal loading on the steel beams of $\varepsilon_{\theta}=7.405 \%$ according to DIN EN 1994-1-2, 3.3.1 [32]. In load case 2, the beams remain cold with $20^{\circ} \mathrm{C}$, the full stiffness of $E_{a}=210000 \mathrm{~N} / \mathrm{mm}^{2}$ is used and no thermal loading is applied on the steel profiles. The substitute thermal loading on the slab can be found in Section 4.2.3, Table 4.8. The values for this example are assembled in Table 8.1.

Table 8.1: Substitute thermal loading on slab of worked example

\begin{tabular}{|l|c|c|}
\hline Direction of sheeting & $\varepsilon_{\theta, \text { subs }}[\%$ o $]$ & $\kappa_{\theta, \text { subs }}[1 / \mathrm{km}]$ \\
\hline Longitudinal & 1.916 & 55.50 \\
\hline Transversal & 1.649 & 44.08 \\
\hline
\end{tabular}

The numerical system is solved as geometrically non-linear to take into account membrane forces that arise due to large deformations. Material non-linearities are already included in the system and do not need to be considered further in the solving procedure. Basically, every cross-section in the slab system has to be designed for its internal forces. Specialised finite element software packages for structural engineering, such as Sofistik, often include routines that perform the designing automatically and the required reinforcement amount is determined at every point in the structure. In order to demonstrate the design procedure and to illustrate the results of this example, the results at the points marked in Figure 8.2 are determined. The vertical deformations $w$, bending moments $m$, membrane forces $n$, shear forces $v$ and the resulting required reinforcement amount $a_{s, r e q}$ are assembled for load case 1 in Table 8.2 and for load case 2 in Table 8.3.

Table 8.2: Vertical deformations, internal forces and required top reinforcement, LC1 hot beams

\begin{tabular}{|c|c|c|c|c|c|c|}
\hline Point & Direction & $\mathrm{w}$ & $\mathrm{m}$ & $\mathrm{n}$ & $\mathrm{v}$ & $a_{s, \text { req }}$ \\
\hline & & {$[\mathrm{mm}]$} & {$[\mathrm{kNm} / \mathrm{m}]$} & {$[\mathrm{kN} / \mathrm{m}]$} & {$[\mathrm{kN} / \mathrm{m}]$} & {$\left[\mathrm{cm}^{2} / \mathrm{m}\right]$} \\
\hline 1 & $\mathrm{y}$ & 154 & -2.42 & 175 & - & $\mathbf{2 . 7 7}$ \\
\hline 2 & $\mathrm{x}$ & 154 & -0.71 & 90.4 & - & $\mathbf{1 . 2 9}$ \\
\hline 3 & $\mathrm{x}$ & 49 & -10.7 & 101 & 10.3 & 6.08 \\
\hline 4 & $\mathrm{y}$ & 43 & -9.26 & 171 & 8.36 & $\mathbf{4 . 8 2}$ \\
\hline 5 & $\mathrm{y}$ & - & -5.47 & 633 & 3.58 & $\mathbf{8 . 0 9}$ \\
\hline 6 & $\mathrm{x}$ & - & -9.10 & 702 & 12.3 & $\mathbf{1 2 . 7}$ \\
\hline 7 & $\mathrm{y}$ & 93 & -2.54 & 144 & - & $\mathbf{2 . 5 3}$ \\
\hline 8 & $\mathrm{x}$ & 93 & -1.21 & 141 & - & $\mathbf{2 . 1 0}$ \\
\hline 9 & $\mathrm{x}$ & 11 & -10.1 & 144 & 10.0 & 6.35 \\
\hline
\end{tabular}

The decisive results are marked in bold. It can be seen that it is not obvious which load case leads to the largest forces. It is notable that the deformations at the slab centres are larger if the beams remain cold. The reason for this is that the slab is stretched by the restrained thermal elongation of the beams. 
Table 8.3: Vertical deformations, internal forces and required top reinforcement, LC2 cold beams

\begin{tabular}{|c|c|c|c|c|c|c|}
\hline Point & Direction & $\mathrm{w}$ & $\mathrm{m}$ & $\mathrm{n}$ & $\mathrm{v}$ & $a_{s, \text { req }}$ \\
\hline & & {$[\mathrm{mm}]$} & {$[\mathrm{kNm} / \mathrm{m}]$} & {$[\mathrm{kN} / \mathrm{m}]$} & {$[\mathrm{kN} / \mathrm{m}]$} & {$\left[\mathrm{cm}^{2} / \mathrm{m}\right]$} \\
\hline 1 & $\mathrm{y}$ & 225 & -2.14 & 25.7 & - & 0.82 \\
\hline 2 & $\mathrm{x}$ & 225 & 3.36 & 40.4 & - & 0.23 \\
\hline 3 & $\mathrm{x}$ & 22 & -17.0 & 52.3 & 17.4 & $\mathbf{9 . 4 6}$ \\
\hline 4 & $\mathrm{y}$ & 17 & -14.7 & -29.6 & 14.5 & 4.05 \\
\hline 5 & $\mathrm{y}$ & - & -5.60 & 132 & 1.71 & 2.74 \\
\hline 6 & $\mathrm{x}$ & - & -6.19 & 188 & 4.05 & 5.05 \\
\hline 7 & $\mathrm{y}$ & 150 & -0.73 & -19.6 & - & 0.39 \\
\hline 8 & $\mathrm{x}$ & 150 & 1.52 & 19.4 & - & 0.21 \\
\hline 9 & $\mathrm{x}$ & 3 & -14.2 & -7.90 & 14.9 & $\mathbf{7 . 1 9}$ \\
\hline
\end{tabular}

\subsection{Design of the slab}

The design procedure shall be demonstrated at two points: point 4 for a cross-section in longitudinal direction and point 3 in transversal direction. The used cross-section at point 4 is shown in Figure 8.3.

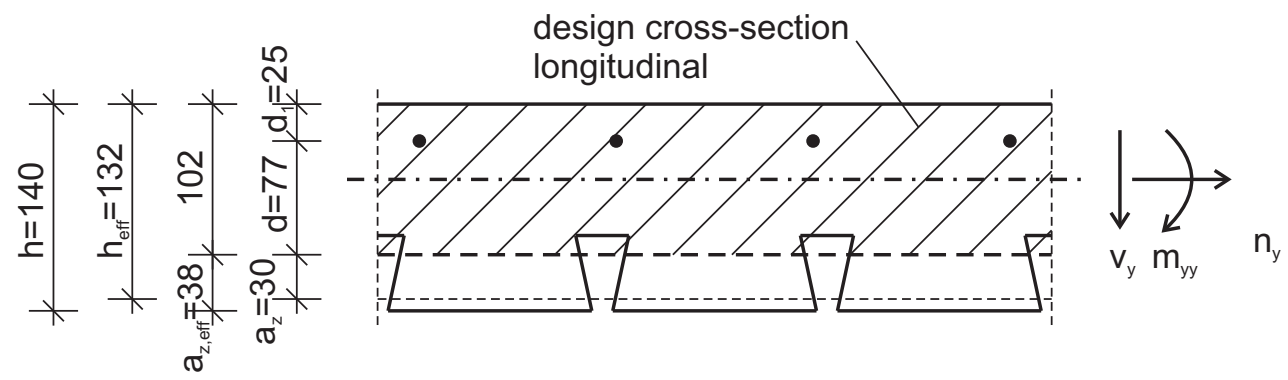

Fig. 8.3: Cross-section for design in longitudinal direction of steel sheeting

As described in Section 6.1.2, a rectangular cross-section is considered with a depth of:

$$
h_{\text {desi }}=h-a_{z, e f f}=140-38=102 \mathrm{~mm}
$$

The thickness of the damaged zone $a_{z, \text { eff }}$ can be found in Table 6.3. The required reinforcement amount will be designed with the method of non-dimensional moment parameters according to [55]. The bending moment about the axis of the tensioned reinforcement is:

$$
m_{E d, s}=m_{E d}-n_{E d} z_{s 1}=9.26-171(0.102 / 2-0.025)=4.81 \mathrm{kNm} / \mathrm{m}
$$

Since the reinforcement lies on the tensioned side of the neutral axis, the bending moment is assumed positively. The non-dimensional moment parameter is calculated as follows:

$$
\mu_{E d, s}=\frac{m_{E d, s}}{b d^{2} f_{c d}}=\frac{0.00481}{1.0 \cdot 0.077^{2} \cdot 20}=0.0406
$$

A mechanical reinforcement ratio of $\omega=0.0416$ can be obtained with Table 6.3 of [55]. The required reinforcement amount is then calculated as:

$$
a_{s, r e q}=\frac{1}{f_{y}}\left(\omega b d f_{c d}+n_{E d}\right)=\frac{1}{50.0}(0.0416 \cdot 100 \cdot 7.7 \cdot 2.0+171)=4.70 \mathrm{~cm}^{2} / \mathrm{m}
$$


This value differs slightly from the value shown in Table 8.2 due to rounding differences.

The shear resistance is determined according to DIN EN 1992-1-1, 6.2.2 [26]. The values $C_{R d, c}$ and $k_{1}$ are used as specified in the German national annex to DIN EN 1992-1-1.

$$
\begin{aligned}
d & <200 \mathrm{~mm} \Rightarrow k=2.0 \\
\rho_{l} & =\frac{A_{s l}}{b_{w} d}=\frac{4.70}{100 \cdot 7.7}=0.00610 \\
\sigma_{c p} & =\frac{N_{E d}}{A_{c}}=\frac{-0.171}{1.0 \cdot 0.102}=-1.676 \mathrm{~N} / \mathrm{mm}^{2} \\
& <0.2 f_{c d}=0.2 \cdot 20=4.0 \mathrm{~N} / \mathrm{mm}^{2} \\
v_{R d, c} & =\left[C_{R d, c} \mathrm{k}\left(100 \rho_{l} f_{c k}\right)^{\frac{1}{3}}+k_{1} \sigma_{c p}\right] b_{w} d= \\
& =\left[0.15 \cdot 2.0(100 \cdot 0.00610 \cdot 20)^{\frac{1}{3}}+0.12 \cdot(-1.676)\right] \cdot 1.0 \cdot 0.077=0.0377 \mathrm{MN} / \mathrm{m} \\
& \hat{=} 37.7 \mathrm{kN} / \mathrm{m}>v_{E d}=8.36 \mathrm{kN} / \mathrm{m}
\end{aligned}
$$

The shear resistance is larger than the available shear force.

In the transversal direction, the weakest part of the slab is above the ribs. The cross-section shown in Figure 8.4 is used for design. The thickness of the damaged zone $a_{z, \text { trans }}$ is given in Table 6.3. The resulting rectangular cross-section has a remaining depth of $h_{\text {desi }}=74 \mathrm{~mm}$.

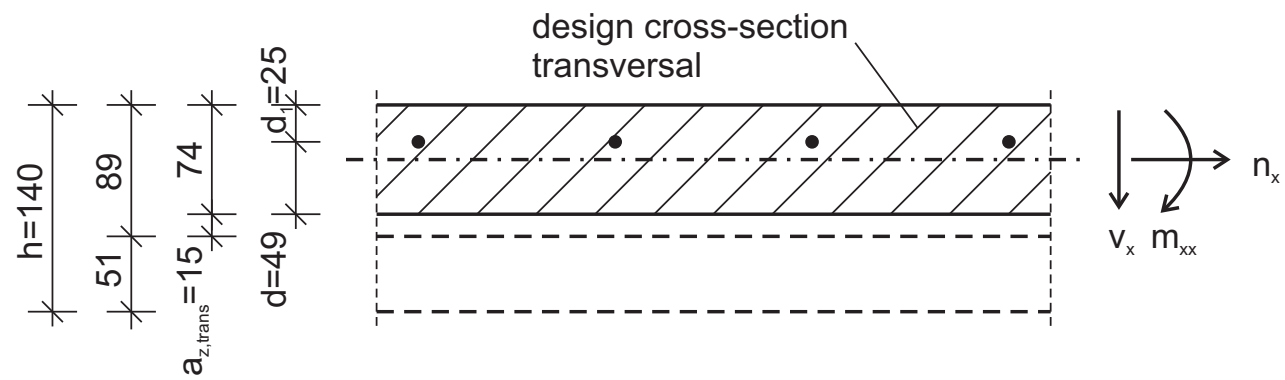

Fig. 8.4: Cross-section for design in transversal direction of steel sheeting

The required amount of reinforcement due to tension and bending at point 3 in Figure 8.2 with the internal forces shown in Table 8.3 is determined as follows:

$$
\begin{aligned}
& m_{E d, s}=m_{E d}-n_{E d} z_{s 1}=17.0-52.3(0.074 / 2-0.025)=16.4 \mathrm{kNm} / \mathrm{m} \\
& \mu_{E d, s}=\frac{m_{E d, s}}{b d^{2} f_{c d}}=\frac{0.0164}{1.0 \cdot 0.049^{2} \cdot 20}=0.3415 \\
& \Rightarrow \omega=0.442 \\
& a_{s, r e q}=\frac{1}{f_{y}}\left(\omega b d f_{c d}+n_{E d}\right)=\frac{1}{50.0}(0.442 \cdot 100 \cdot 4.9 \cdot 2.0+52.3)=9.71 \mathrm{~cm}^{2} / \mathrm{m}
\end{aligned}
$$

The difference from the value in Table 8.3 is again due to rounding differences. The shear resistance is again sufficient with the available reinforcement from the design for bending:

$$
\begin{aligned}
& d<200 \mathrm{~mm} \Rightarrow k=2.0 \\
& \rho_{l}=\frac{A_{s l}}{b_{w} d}=\frac{9.71}{100 \cdot 4.9}=0.0198
\end{aligned}
$$




$$
\begin{aligned}
\sigma_{c p} & =\frac{N_{E d}}{A_{c}}=\frac{-0.0523}{1.0 \cdot 0.074}=-0.707 \mathrm{~N} / \mathrm{mm}^{2} \\
& <0.2 f_{c d}=0.2 \cdot 20=4.0 \mathrm{~N} / \mathrm{mm}^{2} \\
v_{R d, c} & =\left[C_{R d, c} k\left(100 \rho_{l} f_{c k}\right)^{\frac{1}{3}}+k_{1} \sigma_{c p}\right] b_{w} d= \\
& =\left[0.15 \cdot 2.0(100 \cdot 0.0198 \cdot 20)^{\frac{1}{3}}+0.12 \cdot(-0.707)\right] \cdot 1.0 \cdot 0.049=0.0459 \mathrm{MN} / \mathrm{m} \\
& \hat{=} 45.9 \mathrm{kN} / \mathrm{m}>v_{E d}=17.4 \mathrm{kN} / \mathrm{m}
\end{aligned}
$$

It should be noted that the points, which are considered in this example, are not necessarily decisive for determining the required reinforcement amount. The points with the largest forces can lie away from the slab centre or midspan of the intermediate beams.

\subsection{Design of the beams}

The beams are designed according to Section 6.2. Only the protected secondary beams need to be considered since the loading on the primary beams is much smaller in case of fire than under ambient temperature. These are usually sufficiently dimensioned. The intermediate beam at axis B/1-2 will be considered since it suffers the largest loading which can be seen in Figure 8.5.

(1)

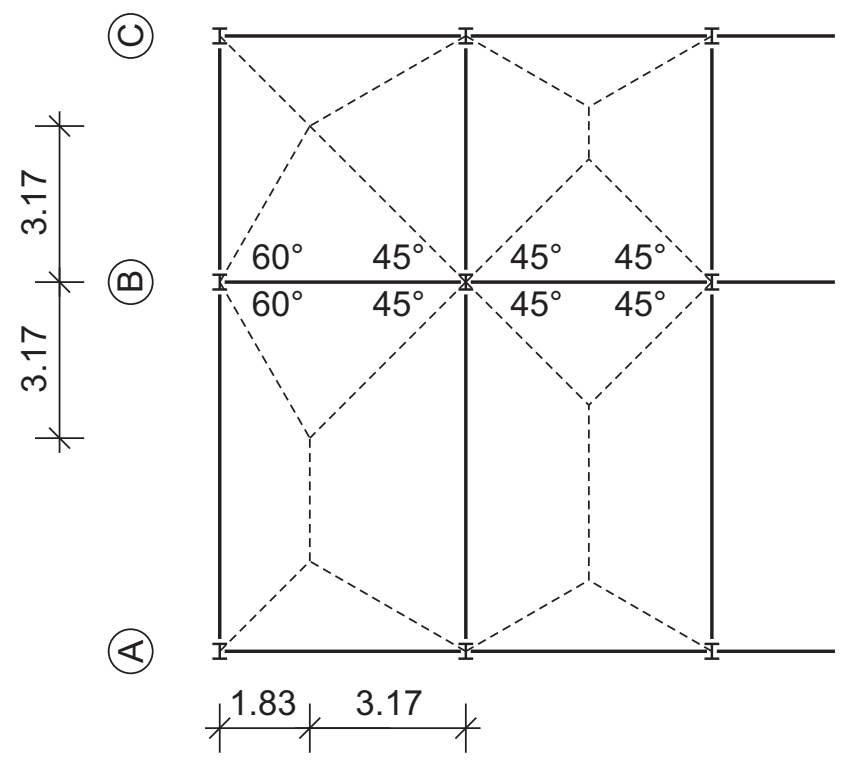

(3)

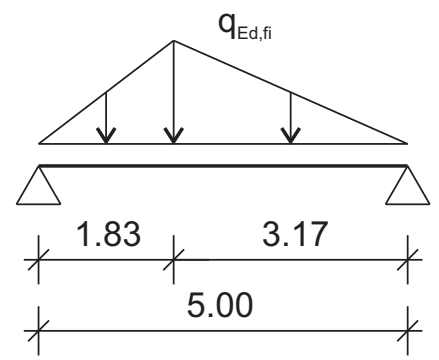

Fig. 8.5: Load distributions on edge beams according to [42]

The bending resistance can be verified through the load ratio under ambient temperature with Equation (6.17):

$$
\frac{M_{E d}}{M_{R d}} \leq \frac{\gamma}{\alpha \eta_{f i}}
$$

This equation is fulfilled for two secondary beams within one slab panel, as is the case in this example, if the load ratio does not exceed 0.34 . The bending resistance under ambient temperature is determined according to DIN EN 1994-1-1 [31]. With a partial shear connection of 50\%, the bending resistance 
becomes:

$$
M_{R d}=159 \mathrm{kNm}
$$

The detailed calculation is not shown here. The distributed load on the slab under ambient temperature is determined as follows:

$$
q_{E d}=\gamma_{g} g_{k}+\gamma_{q} q_{k}=1.35(3.5+2.0)+1.5(2.0+0.8)=11.625 \mathrm{kN} / \mathrm{m}^{2}
$$

The bending moment at midspan and the load ratio is then:

$$
\begin{aligned}
& M_{E d}=\frac{q_{E d} l^{2}}{8}=\frac{2.5 \cdot 11.625 \cdot 5.0^{2}}{8}=90.8 \mathrm{kNm} \\
& \frac{M_{E d}}{M_{R d}}=\frac{90.8}{159}=0.57
\end{aligned}
$$

The load ratio is higher than the simplified limiting value of 0.34 , therefore, the verification needs to be performed more exactly in this case. The bending moment in case of fire at midspan with the triangular load as shown in Figure 8.5 can be calculated as:

$$
\begin{aligned}
q_{E d, f i} & =2 \cdot 6.34 \cdot 3.17=40.2 \mathrm{kN} / \mathrm{m} \\
\Rightarrow M_{E d, f i} & =81.9 \mathrm{kNm}
\end{aligned}
$$

The bending resistance in case of fire is approximately the resistance under ambient temperature multiplied by the reduction factor for the material strength of the steel section according to DIN EN 1993-1-2, Table 3.2 [32]. For a steel temperature of $540^{\circ} \mathrm{C}$ follows:

$$
M_{R d, f i} \approx k_{y, \theta} M_{R d}=0.656 \cdot 159=104 \mathrm{kNm}>M_{E d, f i}=81.9 \mathrm{kNm}
$$

The bending resistance is larger than the bending moment. The beam can bear the load. All other beams have to carry less load and do not need to be verified further. This detailed example has shown that the proposed method of this work can be applied easily for designing new structures in case of fire. 


\section{Summary and recommendations}

In this thesis, a new method has been developed for designing concrete and composite slab systems with partially unprotected secondary beams in case of fire using membrane action. The method is positioned in the research field of membrane action between advanced and simple calculation models. It is based on finite element analyses in order to provide a broad application spectrum but includes radical simplifications for fast and efficient application. The simplifications enable the method to be used with ordinary finite element programs. The amount of reinforcement is designed in the same way as at ambient temperature with internal forces and cross-section design procedures. This thesis makes contribution to the safe and efficient use of membrane action for the design of concrete and composite slab systems in case of fire.

Two full scale fire tests were performed by the author within a research project which formed the basis of this thesis. The main objective of the tests was to generate data to validate design methods and investigating the behaviour of intermediate beams between two slab panels. Both tests confirmed that membrane action enhances the load bearing capacity of concrete and composite slab systems in fire considerably if the secondary beams within a slab panel are unprotected against heating. The first of the two tests revealed an entirely new aspect in the research field of membrane action. A large gaping crack arose nearby the intermediate beam and the slab failed in terms of integrity. The results of another fire test, conducted within the FRACOF project, are used in this thesis in order to cover further construction systems. Like the two tests performed by the author, this slab did not collapse but it also failed in terms of integrity since a large gaping crack occurred at the slab centre. The method proposed in this thesis prevents gaping cracks and this kind of integrity failure by providing sufficient reinforcement.

The thermal analysis is replaced by a substitute thermal loading which can be applied to a simple finite element model. Ordinary shell elements can be used for the slab and simple beam elements for the steel sections. The heating during a fire has two effects in a structure: thermal elongation and material weakening. The substitute thermal loading causes the same thermal elongation as the real temperature distribution in a structure. The material weakening is taken into account by reducing the stiffness of the structure. Thermal analyses on full concrete and composite slabs were performed, compared with test data and simple calculation methods from the literature and used later on for determining the substitute thermal loading. It was shown that the results are in good agreement for full concrete slabs. For composite slabs conservative assumptions were made since the results differ considerably depending on the calculation method and assumptions in the simulation. Two methods were developed for determining the substitute thermal loading: an analytical method that can be used for full concrete slabs and a solution using finite element simulations that can be used generally. Both methods lead to the same results and were validated with an example. Tables were worked out for full concrete slabs and two common composite slab systems that allow a simple application. Cases which are not covered by the tables can be calculated by the proposed simple functions. The substitute thermal loading on the steel beams can be determined with the specifications in the Eurocodes. 
The effects of the heating on the material behaviour are modelled by assuming a reduced stiffness of the finite element model. The stiffness at the ultimate limit state is determined before simulation and a linear elastic material is used. This procedure enables non-linear material calculations to be avoided which are the main reason for excessive computing times and numerical instabilities. The stiffness of the slabs is reduced in two ways: cross-section softening due to reduction of the Young's modulus of the concrete and stiffness reduction due to concrete cracking. It could be shown that the cross-section softening can be taken into account with a simple reduction factor depending on the temperature at the slab centre. The concrete cracking is considered with tension stiffening assumptions which were adopted from ambient temperature approaches. The stiffness reduction of the beams can, like the thermal elongation, be determined with specifications in the Eurocodes.

The finite element model with the reduced stiffness and applied substitute thermal loading is solved as geometrically non-linear but with linear material laws. Internal forces can be determined with this model that are further used to design the required reinforcement amount in the slabs. Design procedures from the Eurocodes were discussed in this thesis and simplified for the requirements of the new method. The cross-section design methods for concrete slabs under ambient temperature can be used to determine the required reinforcement amount for a force combination of bending, axial and shear forces. A very simple method was adopted from the DASt project for verifying the load bearing capacity of the edge beams considering the load level under ambient temperature.

The new method was validated on fire tests. Both the tests performed by the author and the test conducted in the FRACOF project were used. The modelled vertical displacements of the slab were in good agreement for all three tests. At the FRACOF test the simulated displacements also matched the test data for the edge beams. At the two Munich fire tests these displacements are slightly smaller since the stiffness of the shear connections between the edge beams and the L-profiles used as formwork around the edges were difficult to model exactly. Such additional parts as the L-profiles would be conservatively neglected when designing new structures. It is therefore not important to reproduce these results exactly and the correctness of the new method could be confirmed.

A worked example was included to illustrate the simple application of the new method. It was shown that particularly above intermediate beams and around columns considerably more reinforcement is required than predicted with the design methods currently available. This confirms that not enough reinforcement was available in the first Munich fire test which caused the large crack at the intermediate beam to occur. If the reinforcement amount is placed in a slab which is determined by the method of this thesis, the occurrence of such cracks is prevented.

Some assumptions were made in this thesis which require further research. Tension stiffening effects were taken into account using the approaches for ambient temperature since no research results are available for elevated temperatures. The validation of the proposed method on the fire tests indicates that the tension stiffening assumptions are correct but this should be confirmed further. Furthermore, the cross-sections above intermediate beams with the large amount of reinforcement must be proven with experiments to provide enough rotational capacity to perform the large rotations. Finally, the bending resistance of composite slabs in the transversal direction was assumed conservatively in this thesis which should be revised. Despite these conservative assumptions, the presented method enables a simple and efficient design of slab systems in case of fire. 


\section{A Worked example for the substitute thermal loading of a full concrete slab}

$\mathrm{h}=12 \mathrm{~cm}, \mathrm{R} 30$, Indication and equations see Section 4.2.2.

\begin{tabular}{|c|c|c|c|c|c|c|c|c|}
\hline$z_{b}$ & $\theta$ & $\frac{E_{\theta}}{E_{c, 20}}$ & $\frac{E_{\theta}}{E_{c, 20}} \cdot z_{b}$ & $z_{n}$ & $\frac{E_{\theta}}{E_{c, 20}} \cdot z_{n}^{2}$ & $\varepsilon_{\theta}$ & $\frac{\sigma_{\text {nonl }, \theta}}{E_{c, 20}}$ & $\frac{\sigma_{\text {nonl }, \theta}, \theta}{E_{c, 20}} z_{n}$ \\
\hline$[\mathrm{m}]$ & {$\left[{ }^{\circ} \mathrm{C}\right]$} & {$[-]$} & {$[\mathrm{m}]$} & {$[\mathrm{m}]$} & {$\left[\mathrm{m}^{2}\right]$} & {$[-]$} & {$[-]$} & {$[\mathrm{m}]$} \\
\hline 0.0000 & 733.6 & 0.0250 & 0.0000 & 0.0763 & $1.45 \mathrm{E}-4$ & 0.01400 & $3.49 \mathrm{E}-4$ & 2.67E-5 \\
\hline 0.0024 & 669.0 & 0.0347 & 0.0001 & 0.0739 & $1.89 \mathrm{E}-4$ & 0.01273 & $4.41 \mathrm{E}-4$ & $3.26 \mathrm{E}-5$ \\
\hline 0.0048 & 606.9 & 0.0440 & 0.0002 & 0.0715 & $2.25 \mathrm{E}-4$ & 0.01042 & $4.58 \mathrm{E}-4$ & $3.28 \mathrm{E}-5$ \\
\hline 0.0072 & 554.9 & 0.0698 & 0.0005 & 0.0691 & 3.33E-4 & 0.00874 & $6.10 \mathrm{E}-4$ & 4.22E-5 \\
\hline 0.0096 & 505.8 & 0.0968 & 0.0009 & 0.0667 & $4.30 \mathrm{E}-4$ & 0.00735 & $7.11 \mathrm{E}-4$ & $4.74 \mathrm{E}-5$ \\
\hline 0.0120 & 463.5 & 0.1322 & 0.0016 & 0.0643 & $5.46 \mathrm{E}-4$ & 0.00628 & $8.30 \mathrm{E}-4$ & $5.33 \mathrm{E}-5$ \\
\hline 0.0144 & 424.0 & 0.1669 & 0.0024 & 0.0619 & $6.39 \mathrm{E}-4$ & 0.00539 & $8.99 \mathrm{E}-4$ & $5.56 \mathrm{E}-5$ \\
\hline 0.0168 & 389.1 & 0.2006 & 0.0034 & 0.0595 & $7.10 \mathrm{E}-4$ & 0.00468 & $9.38 \mathrm{E}-4$ & $5.58 \mathrm{E}-5$ \\
\hline 0.0192 & 356.8 & 0.2381 & 0.0046 & 0.0571 & $7.75 \mathrm{E}-4$ & 0.00408 & $9.71 \mathrm{E}-4$ & $5.54 \mathrm{E}-5$ \\
\hline 0.0216 & 327.8 & 0.2717 & 0.0059 & 0.0547 & $8.12 \mathrm{E}-4$ & 0.00358 & $9.73 \mathrm{E}-4$ & $5.32 \mathrm{E}-5$ \\
\hline 0.0240 & 301.1 & 0.3028 & 0.0073 & 0.0523 & $8.27 \mathrm{E}-4$ & 0.00316 & $9.56 \mathrm{E}-4$ & $5.00 \mathrm{E}-5$ \\
\hline \multicolumn{9}{|c|}{$\vdots$} \\
\hline 0.0960 & 42.3 & 0.8956 & 0.0860 & -0.0197 & $3.49 \mathrm{E}-4$ & 0.00020 & $1.81 \mathrm{E}-4$ & $-3.6 \mathrm{E}-6$ \\
\hline 0.0984 & 40.8 & 0.9027 & 0.0888 & -0.0221 & $4.42 \mathrm{E}-4$ & 0.00019 & $1.70 \mathrm{E}-4$ & $-3.8 \mathrm{E}-6$ \\
\hline 0.1008 & 39.4 & 0.9090 & 0.0916 & -0.0245 & $5.47 \mathrm{E}-4$ & 0.00018 & $1.60 \mathrm{E}-4$ & $-3.9 \mathrm{E}-6$ \\
\hline 0.1032 & 38.2 & 0.9146 & 0.0944 & -0.0269 & $6.63 \mathrm{E}-4$ & 0.00017 & $1.51 \mathrm{E}-4$ & $-4.1 \mathrm{E}-6$ \\
\hline 0.1056 & 37.2 & 0.9194 & 0.0971 & -0.0293 & 7.91E-4 & 0.00016 & $1.43 \mathrm{E}-4$ & $-4.2 \mathrm{E}-6$ \\
\hline 0.1080 & 36.3 & 0.9236 & 0.0997 & -0.0317 & $9.30 \mathrm{E}-4$ & 0.00015 & $1.36 \mathrm{E}-4$ & $-4.3 \mathrm{E}-6$ \\
\hline 0.1104 & 35.6 & 0.9271 & 0.1024 & -0.0341 & $1.08 \mathrm{E}-3$ & 0.00014 & $1.31 \mathrm{E}-4$ & $-4.5 \mathrm{E}-6$ \\
\hline 0.1128 & 34.9 & 0.9300 & 0.1049 & -0.0365 & $1.24 \mathrm{E}-3$ & 0.00014 & $1.26 \mathrm{E}-4$ & $-4.6 \mathrm{E}-6$ \\
\hline 0.1152 & 34.5 & 0.9322 & 0.1074 & -0.0389 & $1.41 \mathrm{E}-3$ & 0.00013 & $1.22 \mathrm{E}-4$ & $-4.8 \mathrm{E}-6$ \\
\hline 0.1176 & 34.1 & 0.9338 & 0.1098 & -0.0413 & $1.59 \mathrm{E}-3$ & 0.00013 & $1.20 \mathrm{E}-4$ & $-4.9 \mathrm{E}-6$ \\
\hline \multirow[t]{7}{*}{0.1200} & 33.9 & 0.9348 & 0.1122 & -0.0437 & $1.79 \mathrm{E}-3$ & 0.00013 & $1.18 \mathrm{E}-4$ & $-5.2 \mathrm{E}-6$ \\
\hline & & $\frac{E A}{E_{c, 20}}$ & $\frac{E S_{y}}{E_{c, 20}}$ & $a_{z n}$ & $\frac{E I_{y}}{E_{c, 20}}$ & & $\frac{N_{\theta}}{E_{c, 20}}$ & $\frac{M_{\theta}}{E_{c, 20}}$ \\
\hline & & {$\left[\mathrm{m}^{2}\right]$} & {$\left[\mathrm{m}^{3}\right]$} & {$[\mathrm{m}]$} & {$\left[\mathrm{m}^{4}\right]$} & & {$\left[\mathrm{m}^{2}\right]$} & {$\left[\mathrm{m}^{3}\right]$} \\
\hline & & 0.0722 & 0.00551 & 0.0763 & $5.94 \mathrm{E}-5$ & & $5.71 \mathrm{E}-5$ & $1.94 \mathrm{E}-6$ \\
\hline & & & & & & & $\varepsilon_{\theta, \text { subst }}$ & $\kappa_{\theta, \text { subst }}$ \\
\hline & & & & & & & {$[\% o]$} & {$[1 / \mathrm{km}]$} \\
\hline & & & & & & & 0.0791 & 32.65 \\
\hline
\end{tabular}




\section{B Input files}

\section{B.1 Example 4-2: Short beam}

See Section 4.2.4.

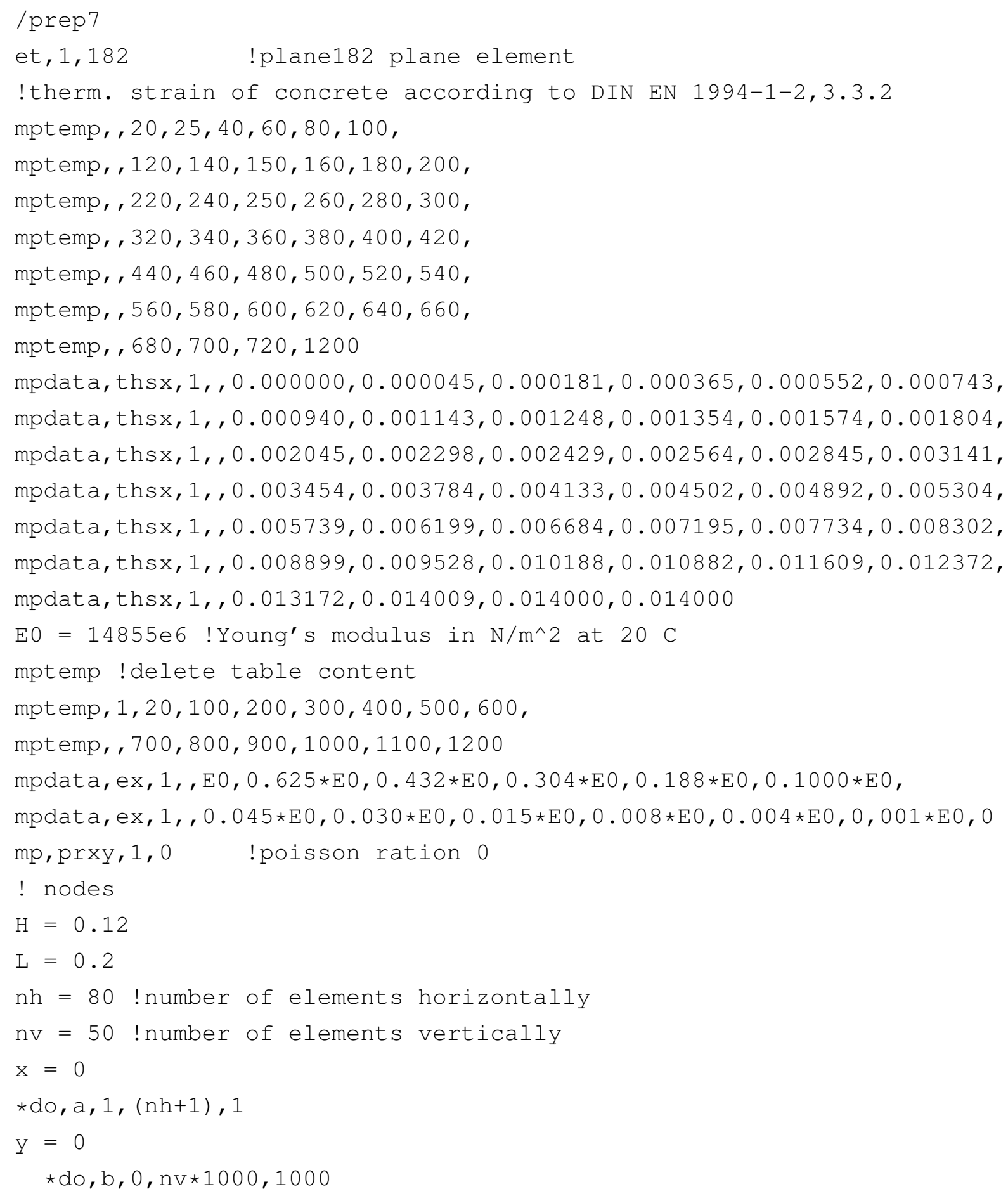




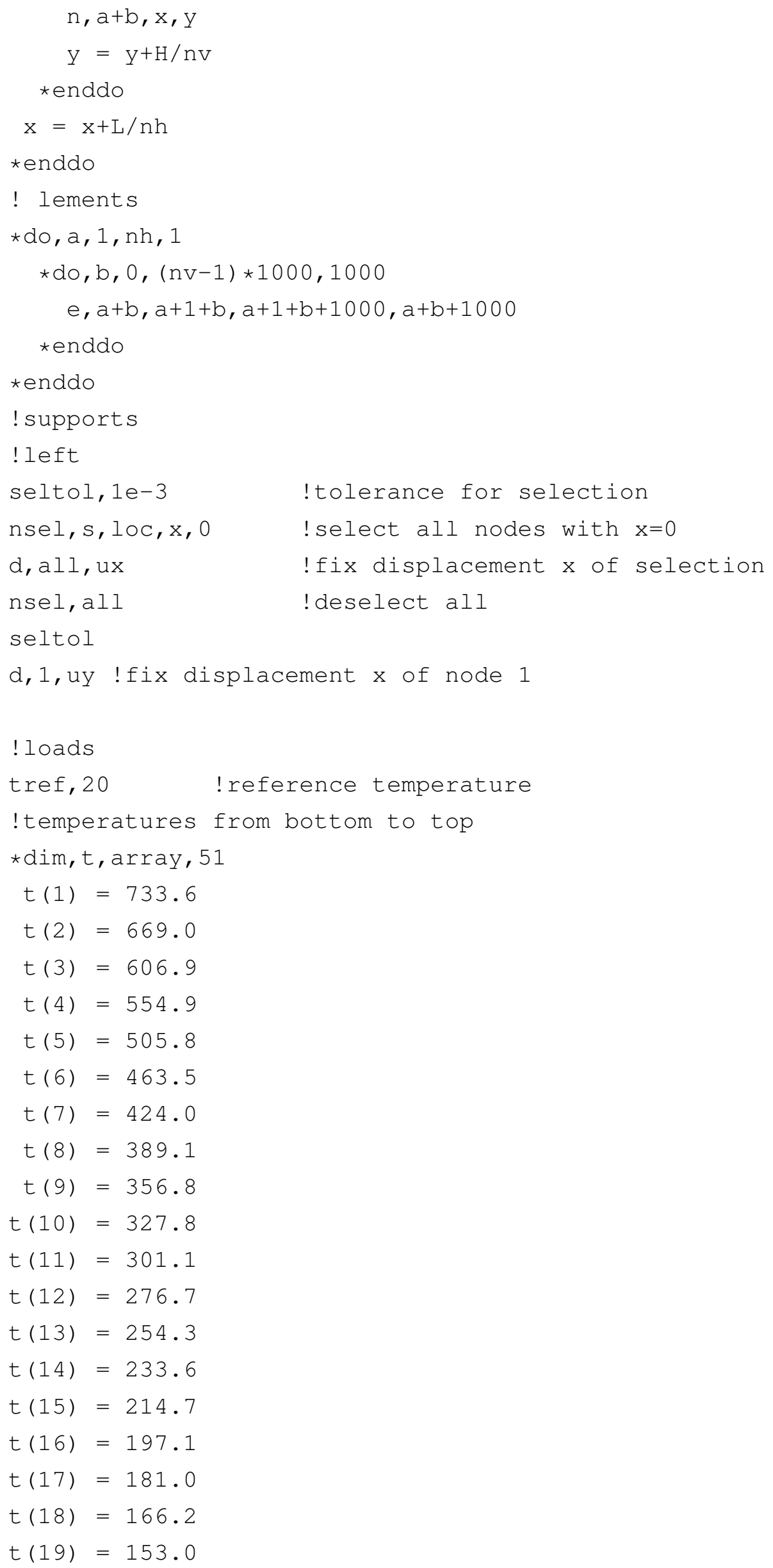


$t(20)=140.9$

$t(21)=130.4$

$t(22)=120.9$

$t(23)=112.7$

$t(24)=105.5$

$t(25)=99.1$

$t(26)=93.0$

$t(27)=87.5$

$t(28)=82.3$

$t(29)=77.5$

$t(30)=73.1$

$t(31)=69.0$

$t(32)=65.1$

$t(33)=61.6$

$t(34)=58.4$

$t(35)=55.4$

$t(36)=52.7$

$t(37)=50.2$

$t(38)=47.9$

$t(39)=45.8$

$t(40)=44.0$

$t(41)=42.3$

$t(42)=40.8$

$t(43)=39.4$

$t(44)=38.2$

$t(45)=37.2$

$t(46)=36.3$

$t(47)=35.6$

$t(48)=34.9$

$t(49)=34.5$

$t(50)=34.1$

$t(51)=33.9$

*do, ny , 0, 50, 1

nsel, s, node, $n y * 1000+1, n y * 1000+1+n h, 1$

bf, all, temp, t (ny+1)

nsel,all

*enddo

finish !finish pre-processor

/ solu

! nonlinear

antype,static ! static analysis

nlgeom, off 
solve

finish

/post 1

! set stress distribution in middle of beam on path

path,schnitt, 2, 50 !define path called "schnitt"

ppath, 1, L/2,0 !define points of path

ppath, $2, \mathrm{~L} / 2, \mathrm{H}$

PDEF, $\mathrm{S}, \mathrm{x}, \mathrm{NOAVG}$ !put stress $\mathrm{SX}$ on path

PDEF, , epel, $x, N O A V G$ !put elastic strain on path

\section{B.2 Example 4-3: Composite beam with shell elements}

See Section 4.3.

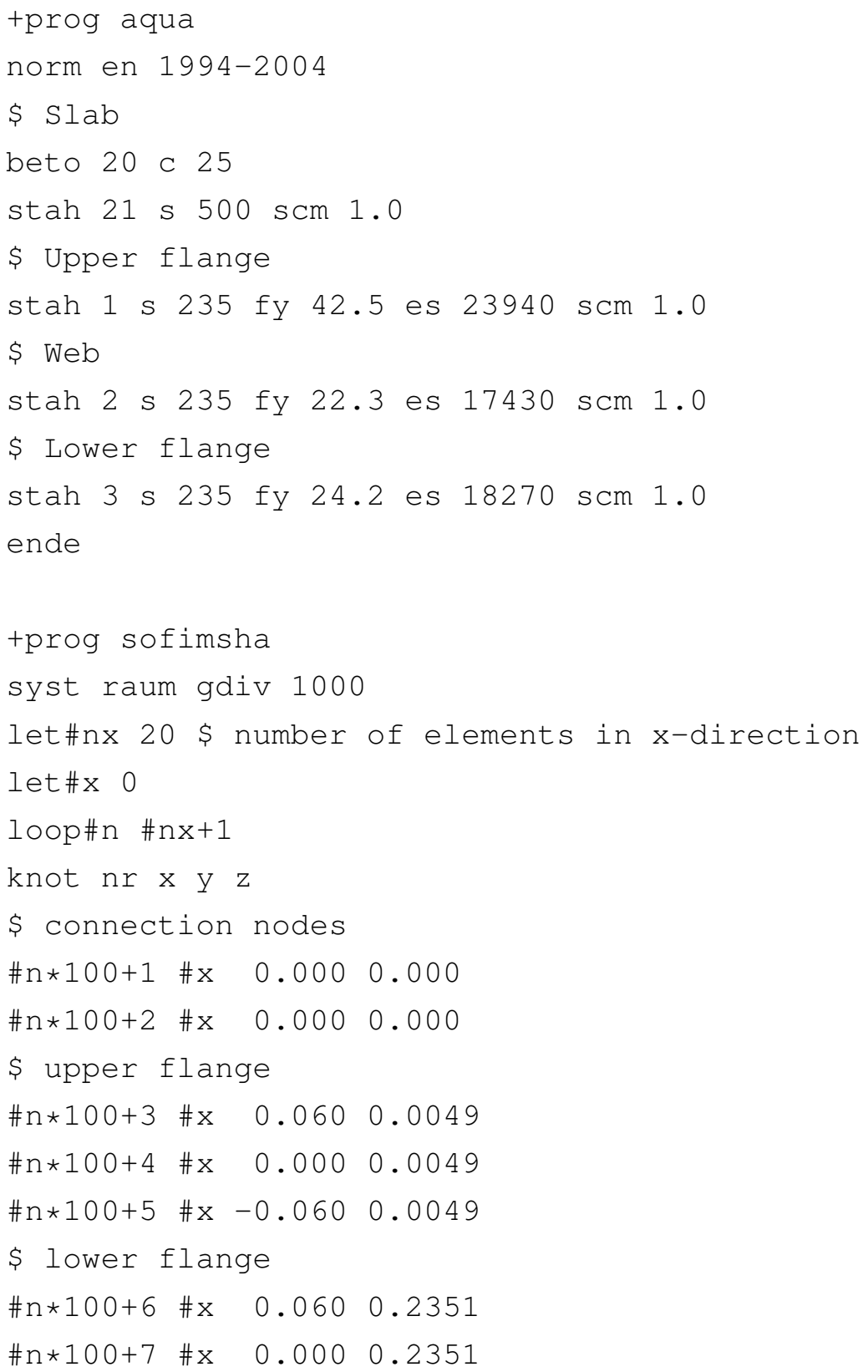




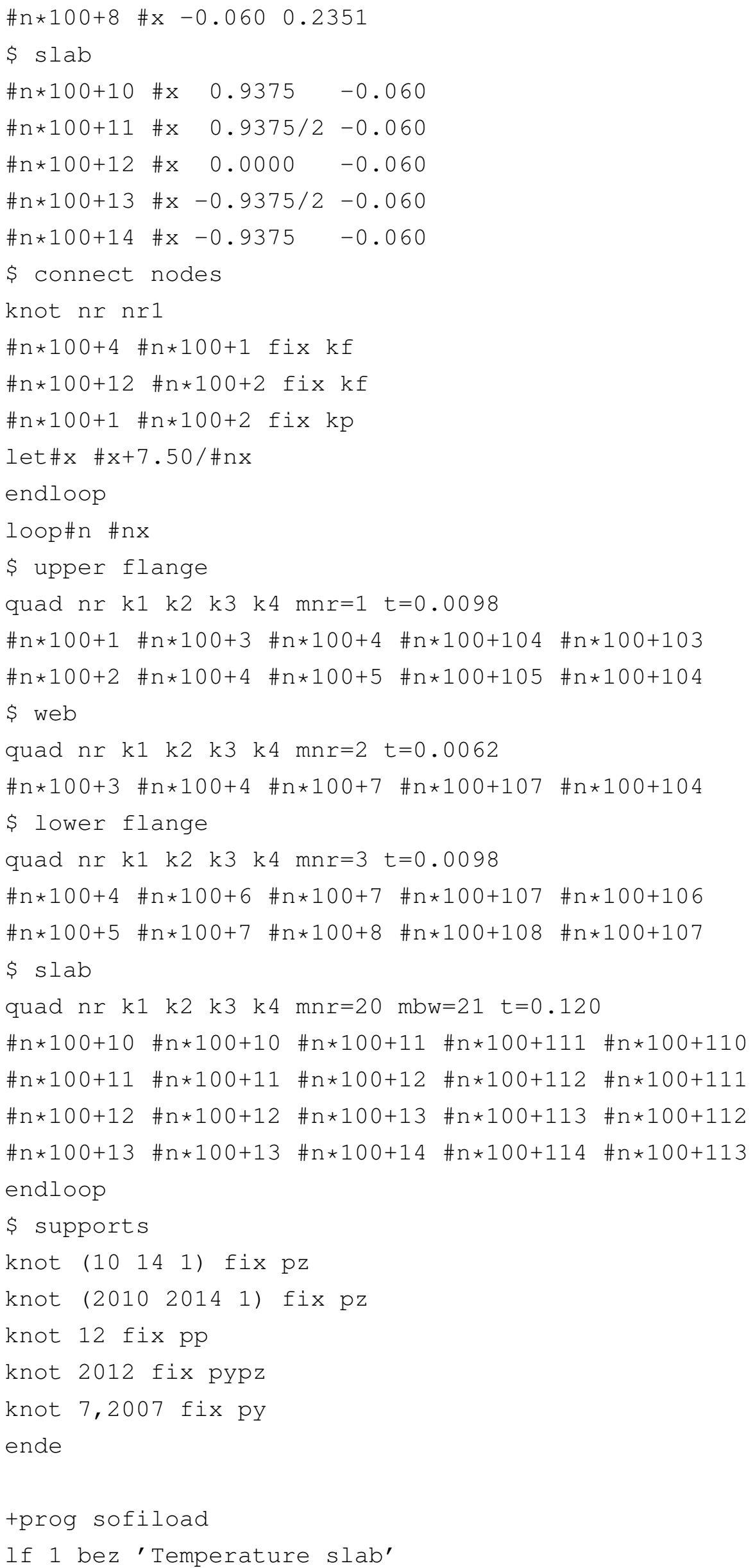




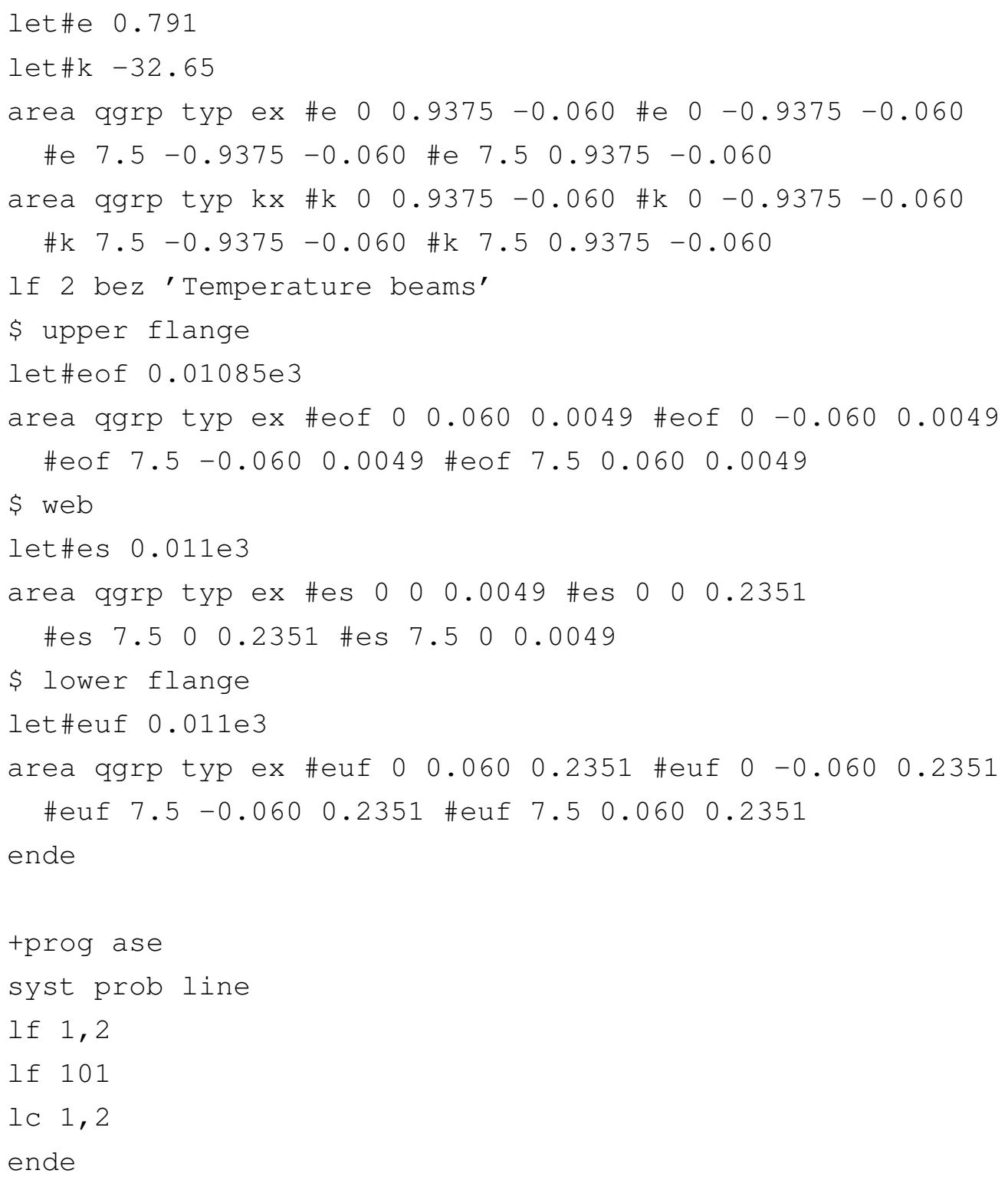

\section{B.3 Example 5-1: Influence of slab stiffness}

See Section 5.2.2.

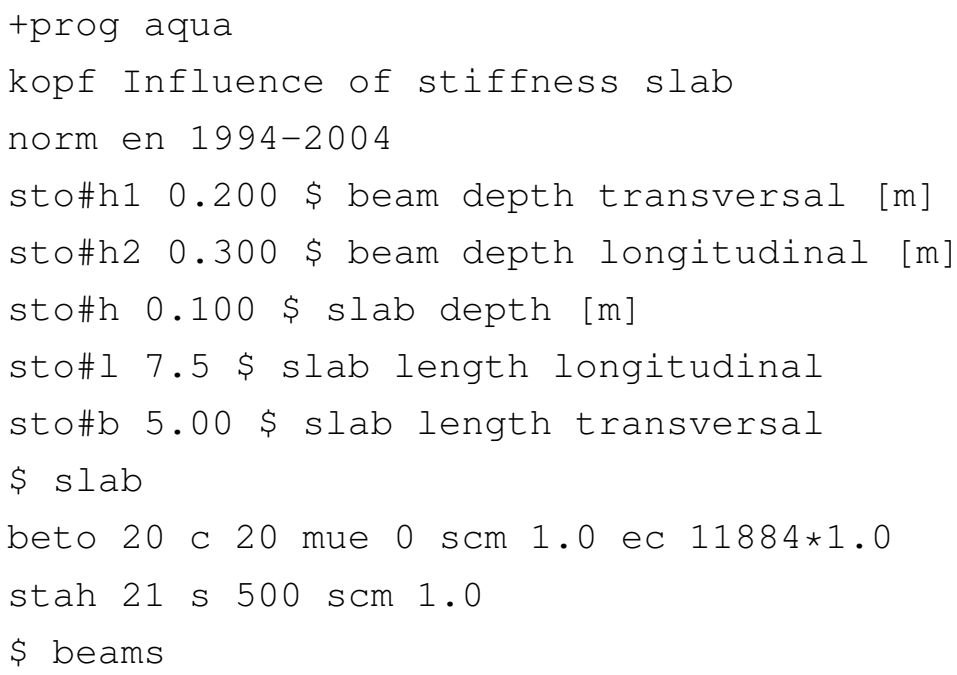




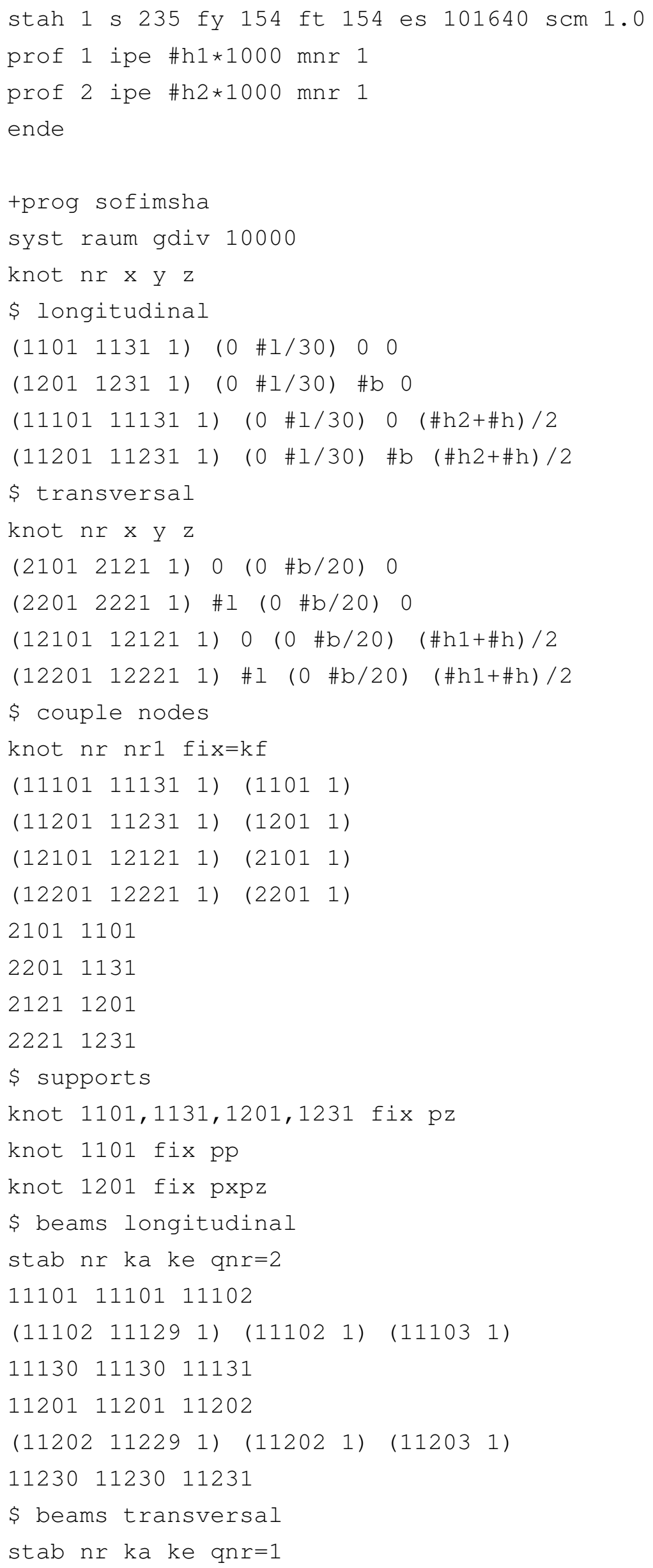


$12101 \quad 12101 \quad 12102$

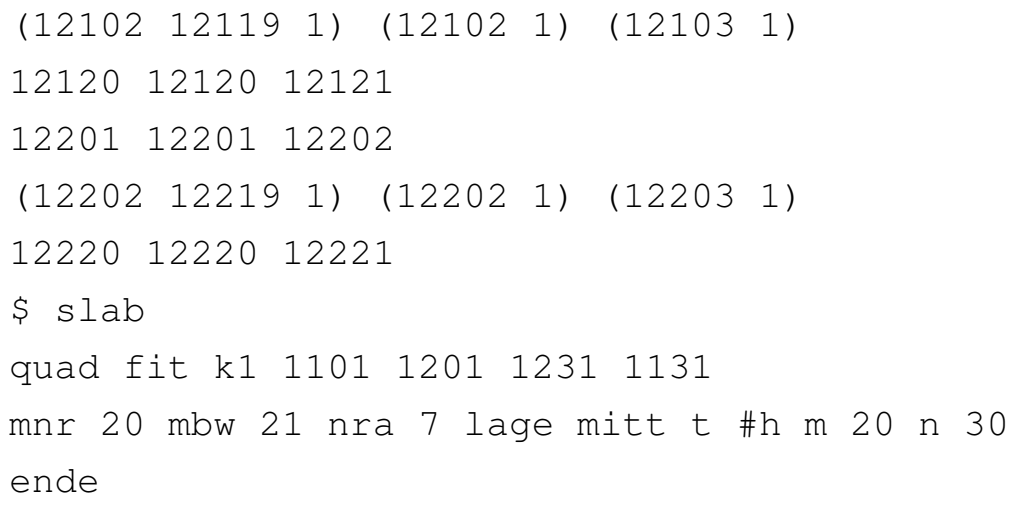




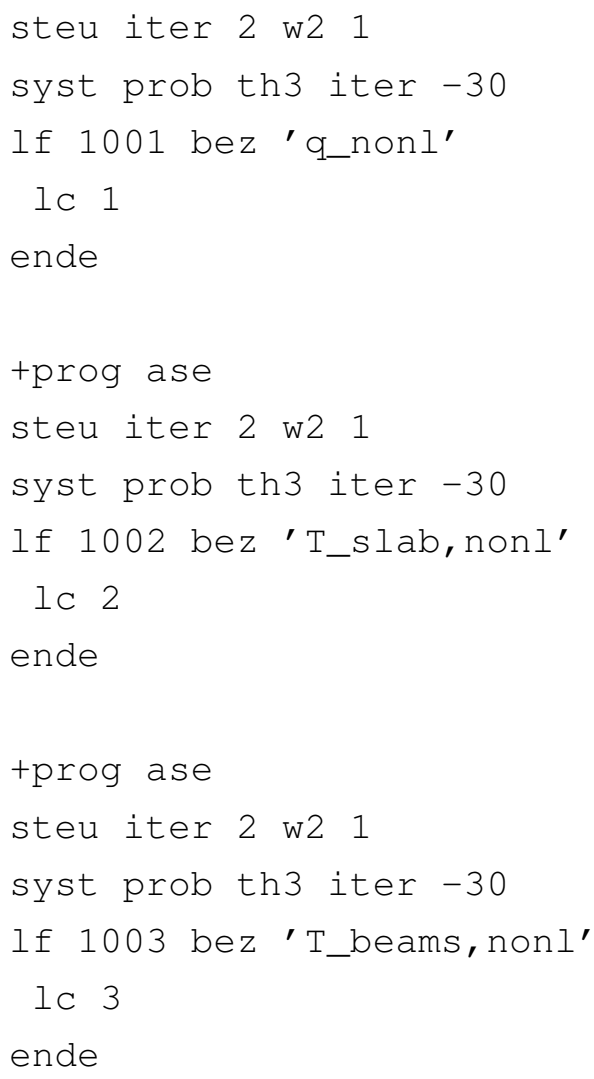

\section{B.4 Example 5-2: Composite beam with beam elements}

See Section 5.3.

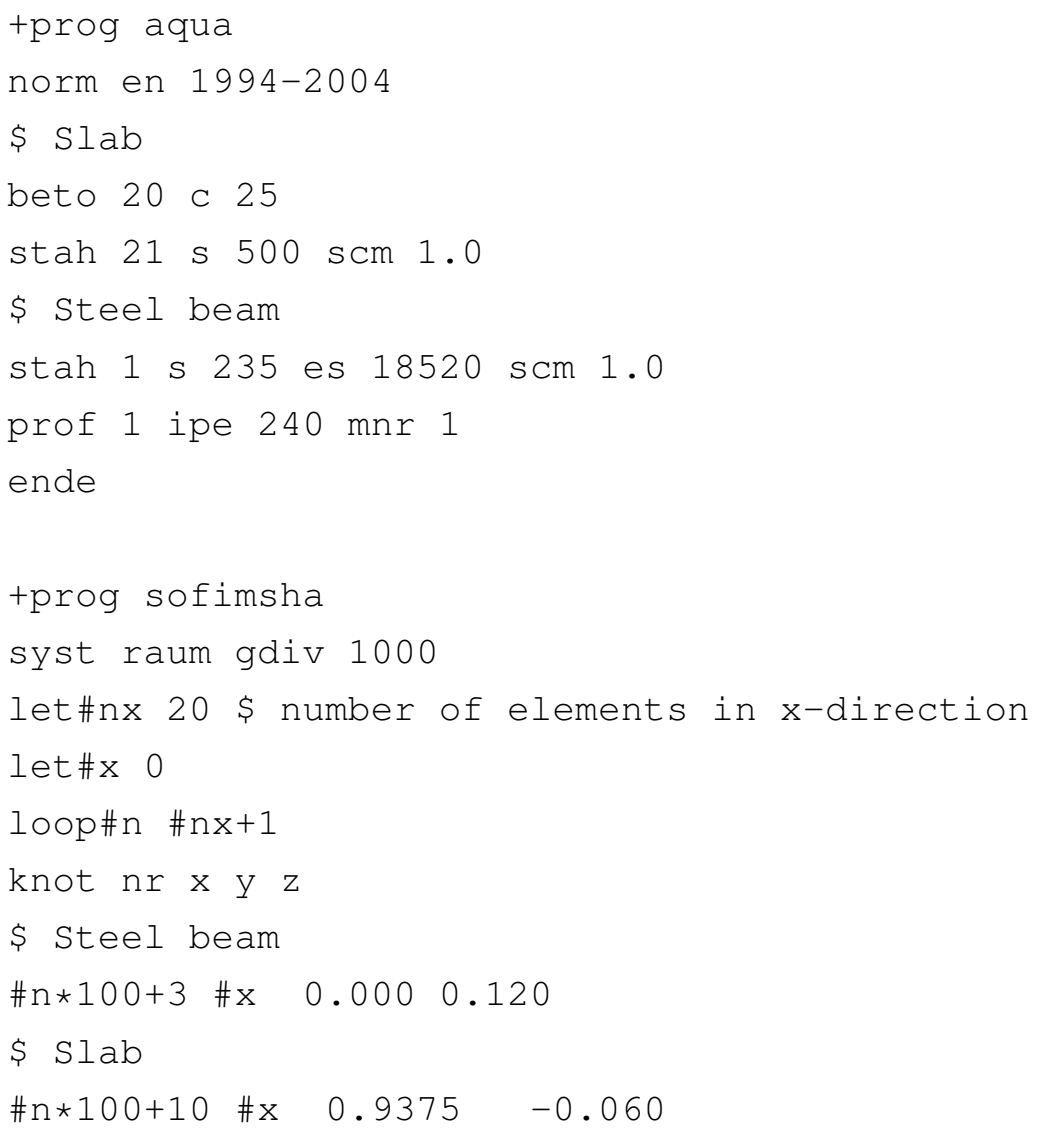




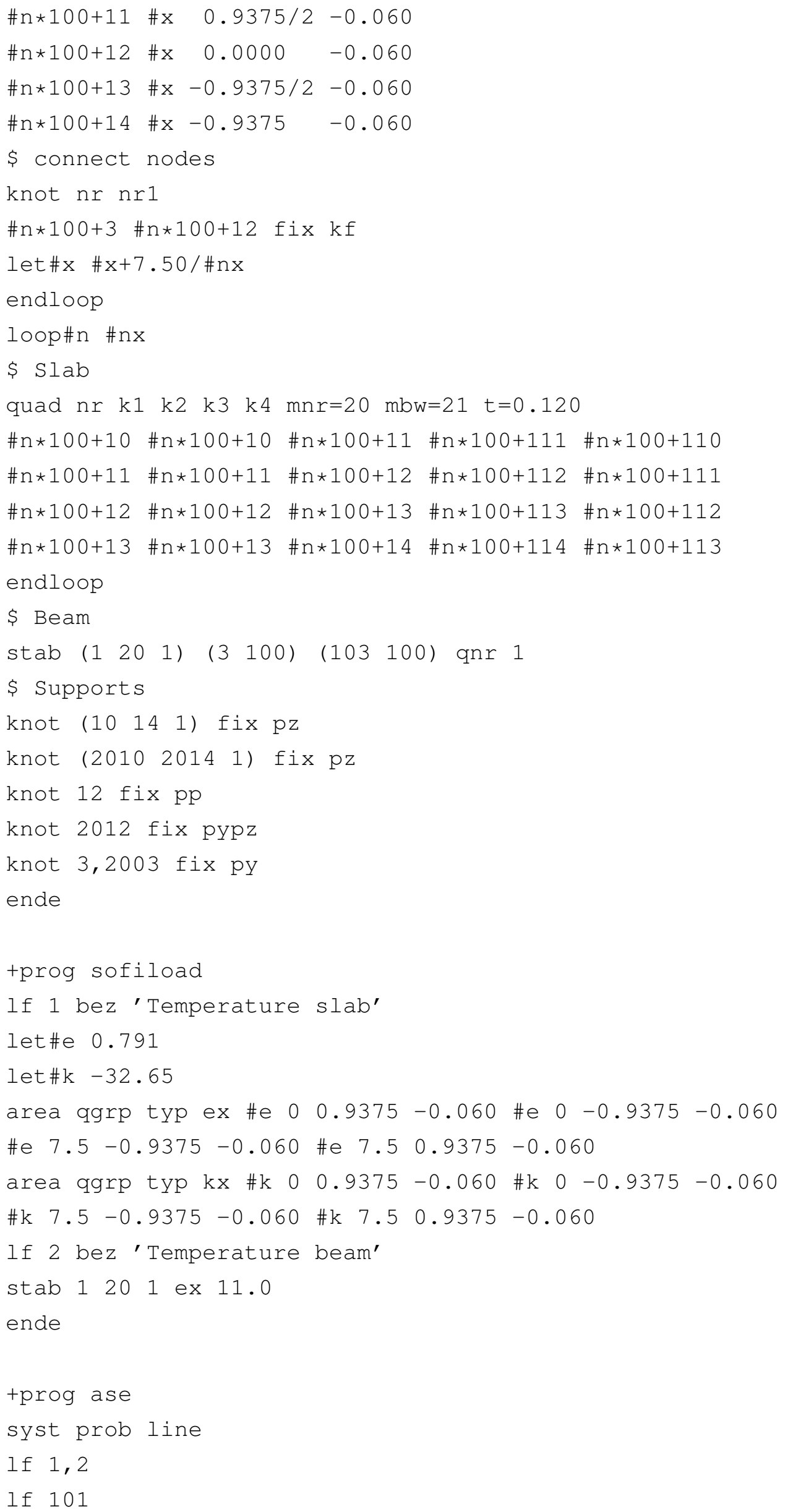


lc 1,2

ende

\section{B.5 Example 6-1: Load bearing characteristics of composite beam}

See Section 6.2.

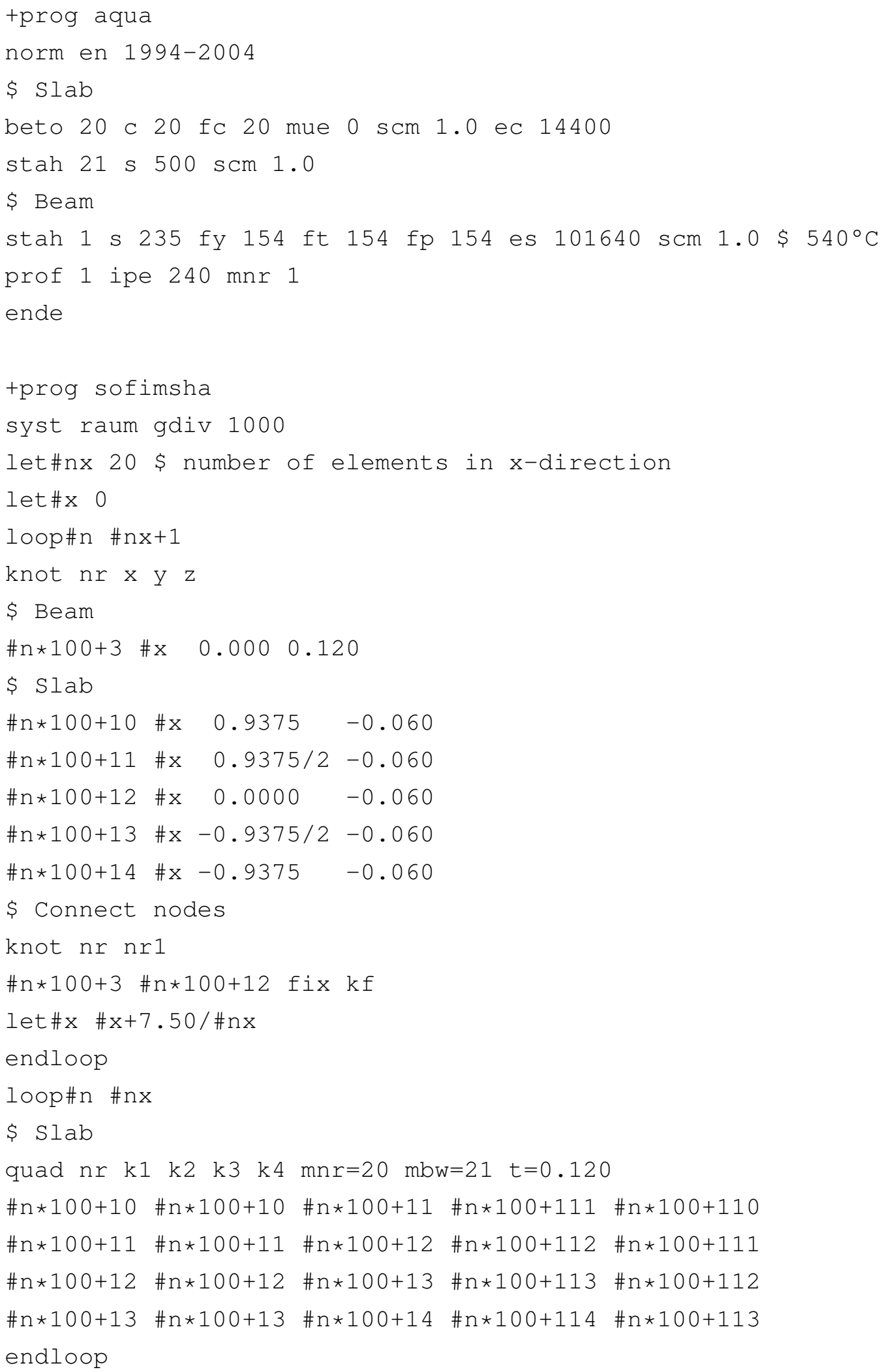




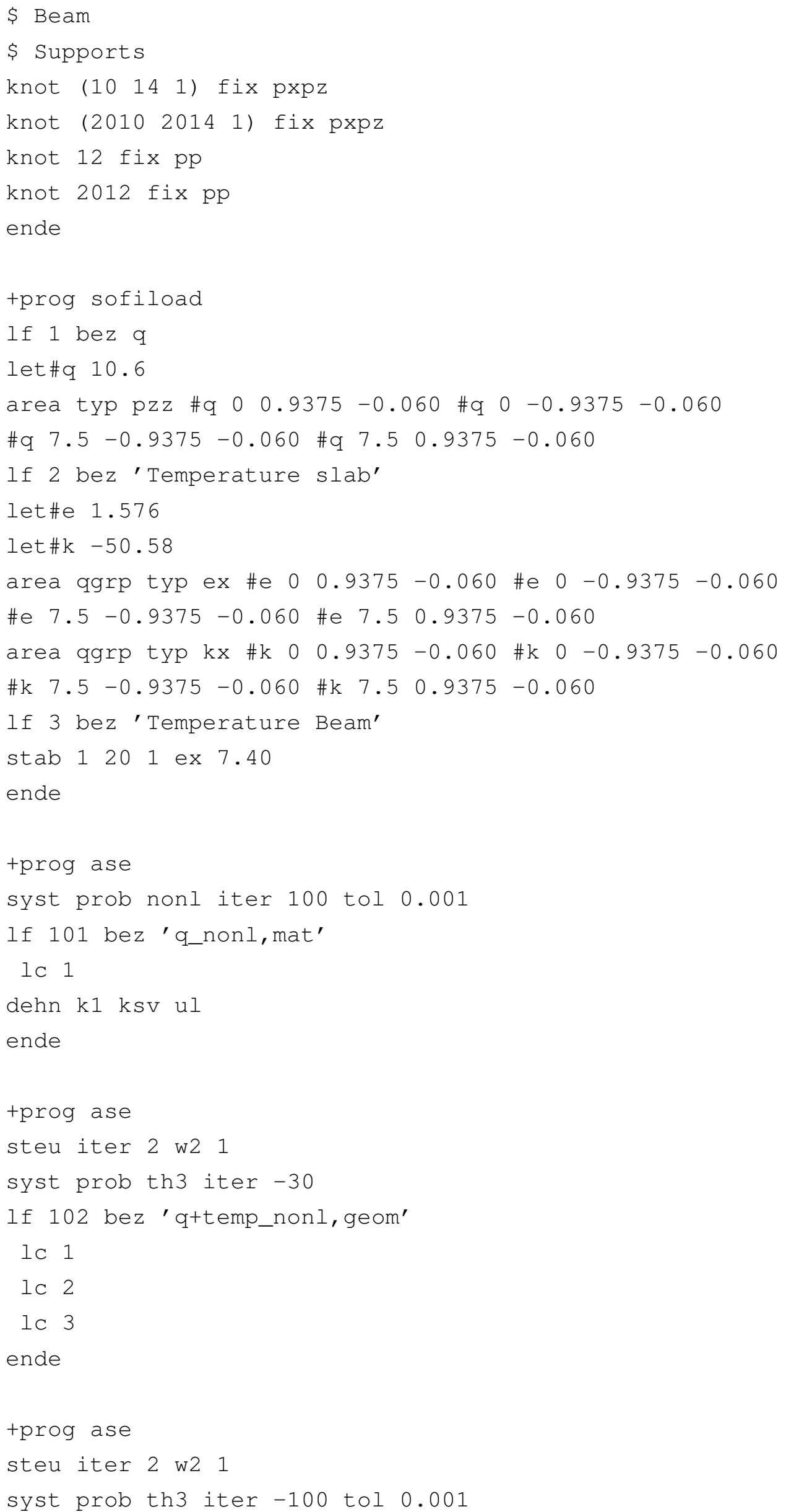




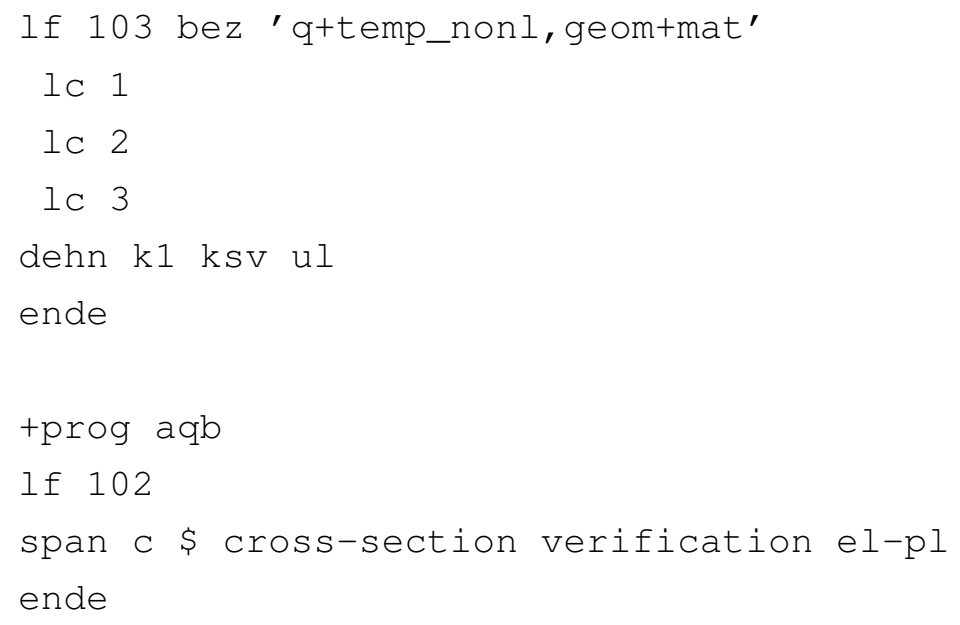

\section{B.6 Validation on first Munich test}

See Section 7.1.

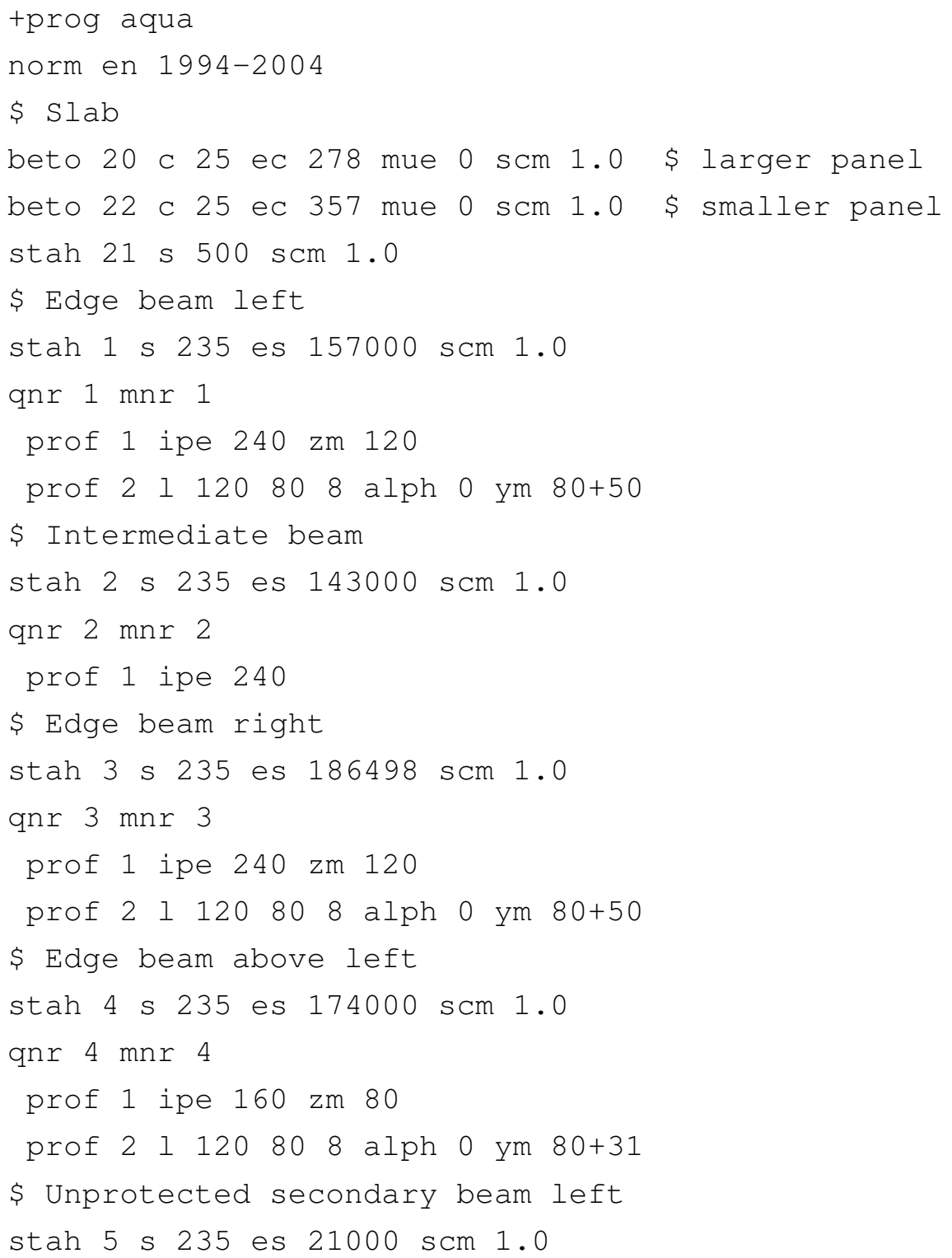




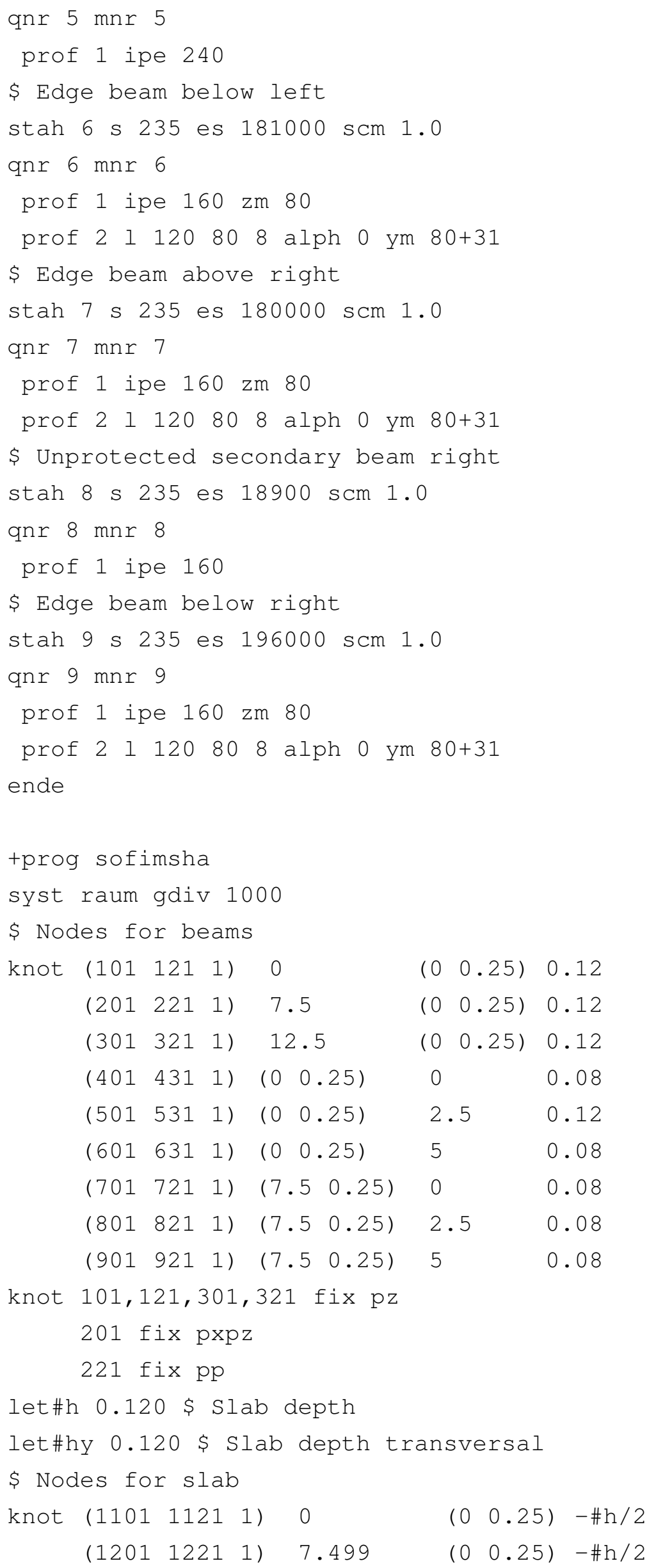


\$ Additional nodes for crack above intermediate beam

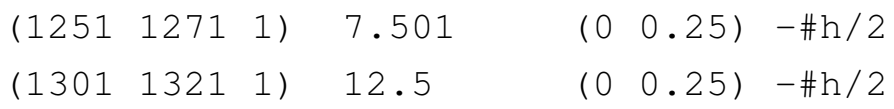

\$ Additional nodes for crack aside intermediate beam

\begin{tabular}{|c|c|c|c|c|c|c|c|}
\hline 331 & 351 & 1) & 7.7 & 49 & 10 & $0.25)$ & $-\# \mathrm{~h} / 2$ \\
\hline 51 & 1381 & 1) & 7.7 & 751 & $(0$ & $0.25)$ & $-\# \mathrm{~h} / 2$ \\
\hline 101 & 1431 & 1) & 10 & $0.25)$ & 0 & & $-\# \mathrm{~h} / 2$ \\
\hline & 1531 & 1) & 10 & $0.25)$ & 2 . & & $-\# \mathrm{~h} / 2$ \\
\hline 501 & 1631 & 1) & 10 & $0.25)$ & 5 & & $-\# \mathrm{~h} / 2$ \\
\hline 701 & 1721 & 1) & $(7$. & $5 \quad 0.25)$ & 0 & & $-\# \mathrm{~h} / 2$ \\
\hline & & 1) & $(7$. & $5 \quad 0.25)$ & 2 . & 5 & $-\# \mathrm{~h} / 2$ \\
\hline & & 1) & ( 7. & $50.25)$ & 5 & & $-\# \mathrm{~h} / 2$ \\
\hline
\end{tabular}

\$ Connect beams

knot nr nrl fix=kf

401101

501111

601121

431201

631221

701201

801211

801211

901221

721301

821311

921321

$\$$ Connect slab to beams

knot nr nrl fix=kf

(1101 1121 1) (101 1 )

$\left(\begin{array}{lll}1301 & 1321 & 1\end{array}\right) \quad(301 \quad 1)$

$\left(\begin{array}{lll}1401 & 1431 & 1\end{array}\right) \quad\left(\begin{array}{lll}401 & 1\end{array}\right)$

$\left(\begin{array}{lll}1501 & 1531 & 1\end{array}\right) \quad(501 \quad 1)$

$\left(\begin{array}{lll}1601 & 1631 & 1\end{array}\right) \quad(6011)$

$\left(\begin{array}{lll}1701 & 1721 & 1\end{array}\right) \quad\left(\begin{array}{ll}701 & 1\end{array}\right)$

$\left(\begin{array}{lll}1801 & 1821 & 1\end{array}\right) \quad(801 \quad 1)$

$\left(\begin{array}{lll}1901 & 1921 & 1\end{array}\right) \quad(901 \quad 1)$

\$ Connect left slab to intermediate beam

knot nr nrl fix=kp

(1202 12201 1) (202 1)

fede (1201 1221 1) (1201 1) (201 1) dy 1 cm lel flie 1.9

knot 1201 fix kf 201

knot 1221 fix kf 221

\$ Connect right slab to intermediate beam 
knot $\mathrm{nr}$ nrl fix $=\mathrm{kp}$

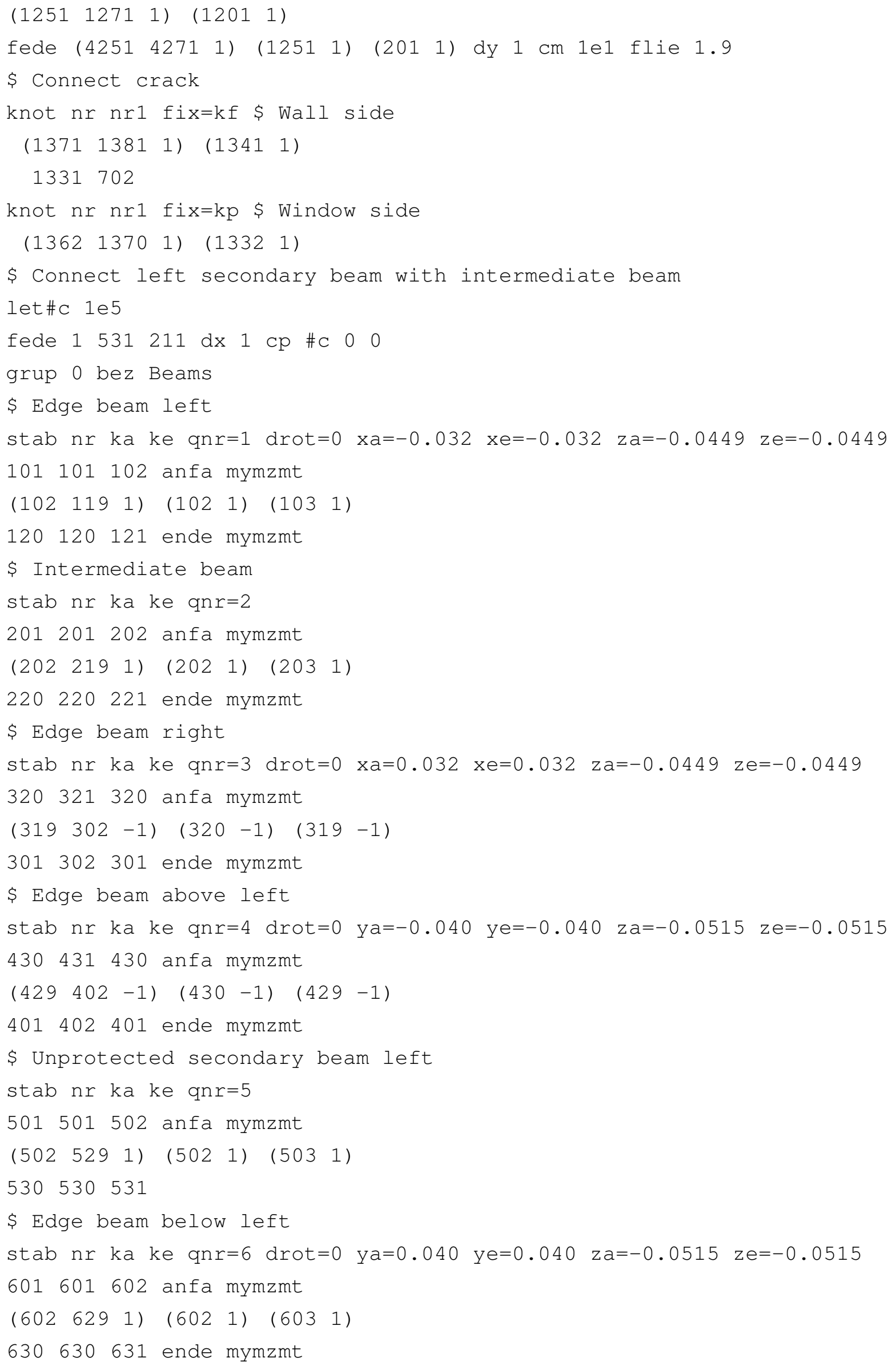


\$ Edge beam above right

stab nr ka ke qn r=7 drot=0 ya=-0.040 ye=-0.040 za=-0.0515 ze=-0.0515

720721720 anfa mymzmt

$\left(\begin{array}{lllll}719 & 702 & -1\end{array}\right)(720-1) \quad(719-1)$

701702701 ende mymzmt

\$ Unprotected secondary beam right

stab nr ka ke qnr $=8$

801801802 anfa mymzmt

$(8028191) \quad(8021) \quad(803 \quad 1)$

820820821 ende mymzmt

\$ Edge beam below right

stab nr ka ke qnr=9 drot=0 ya=0.040 ye=0.040 za=-0.0515 ze=-0.0515

901901902 anfa mymzmt

$\left(\begin{array}{lllll}902 & 919 & 1\end{array}\right)(902 \quad 1) \quad(9031)$

920920921 ende mymzmt

\$ Slab

steu gtol 0.001

grup 1 bez 'Slab left'

quad fit k1 1101112112211201 mnr 20 mbw 21

nra 7 lage mitt $t$ \#h ty \#hy m 20 n 30

grup 2 bez 'Smaller panel left of crack'

quad fit k1 1251127113511331 mnr 22 mbw 21

nra 7 lage mitt $t$ \#h ty \#hy m 20 n 1

grup 3 bez 'Smaller panel right of crack'

quad fit k1 1361138113211301 mnr 22 mbw 21

nra 7 lage mitt $t$ \#h ty \#hy m 20 n 19

ende

+prog sofiload

lf 1 bez $q$

let\#q 5.1

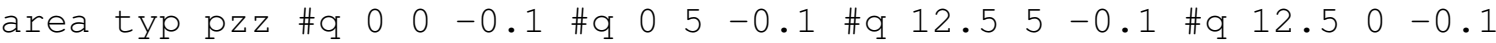

lf 2 bez 'Temperature Slab'

\$ Larger slab

let\#e 1.161 \$ from test data $40 \mathrm{~min}$

let\#k 44.202

area qgrp typ ex \#e 000 \# 0 e 050 \#e 7.550 \#e 7.500

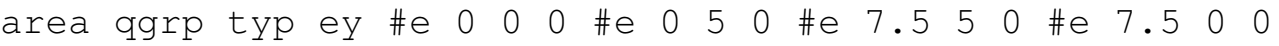

area ggrp typ kx \#k $0 \begin{array}{lllllllllllllllll} & 0 & 0 & \# k & 0 & 5 & 0 & \# k & 7.5 & 5 & 0 & \# k & 7.5 & 0 & 0\end{array}$

area qgrp typ ky \#k $0 \begin{array}{lllllllllllllll} & 0 & \# k & 0 & 5 & 0 & \# k & 7.5 & 5 & 0 & \# k & 7.5 & 0 & 0\end{array}$

\$ Smaller slab

let\#e 1.101 \$ from test data $40 \mathrm{~min}$

let\#k 41.838 


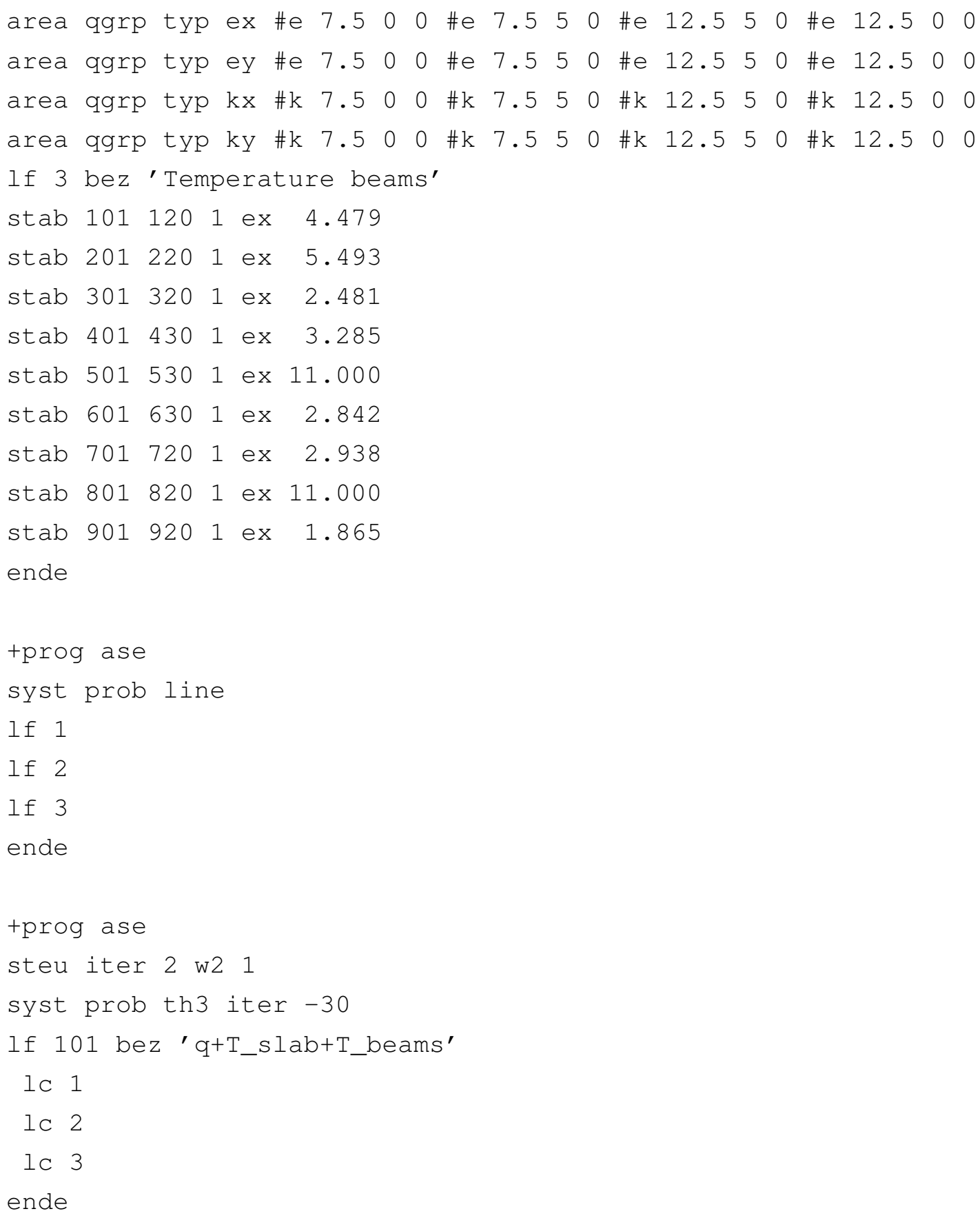

\section{B.7 Validation on second Munich test}

See Section 7.2.

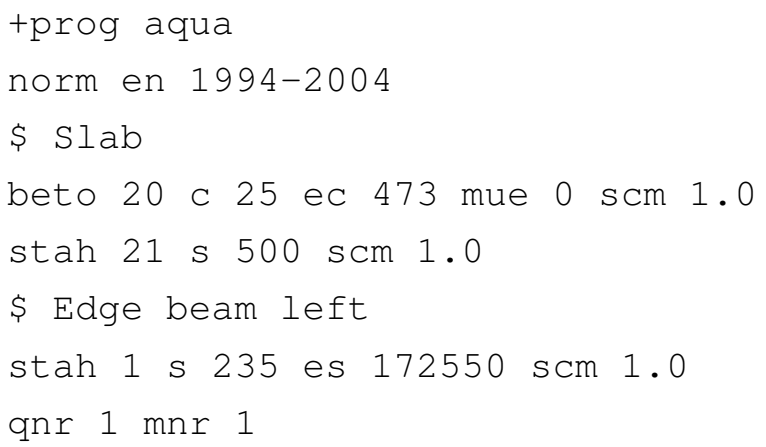




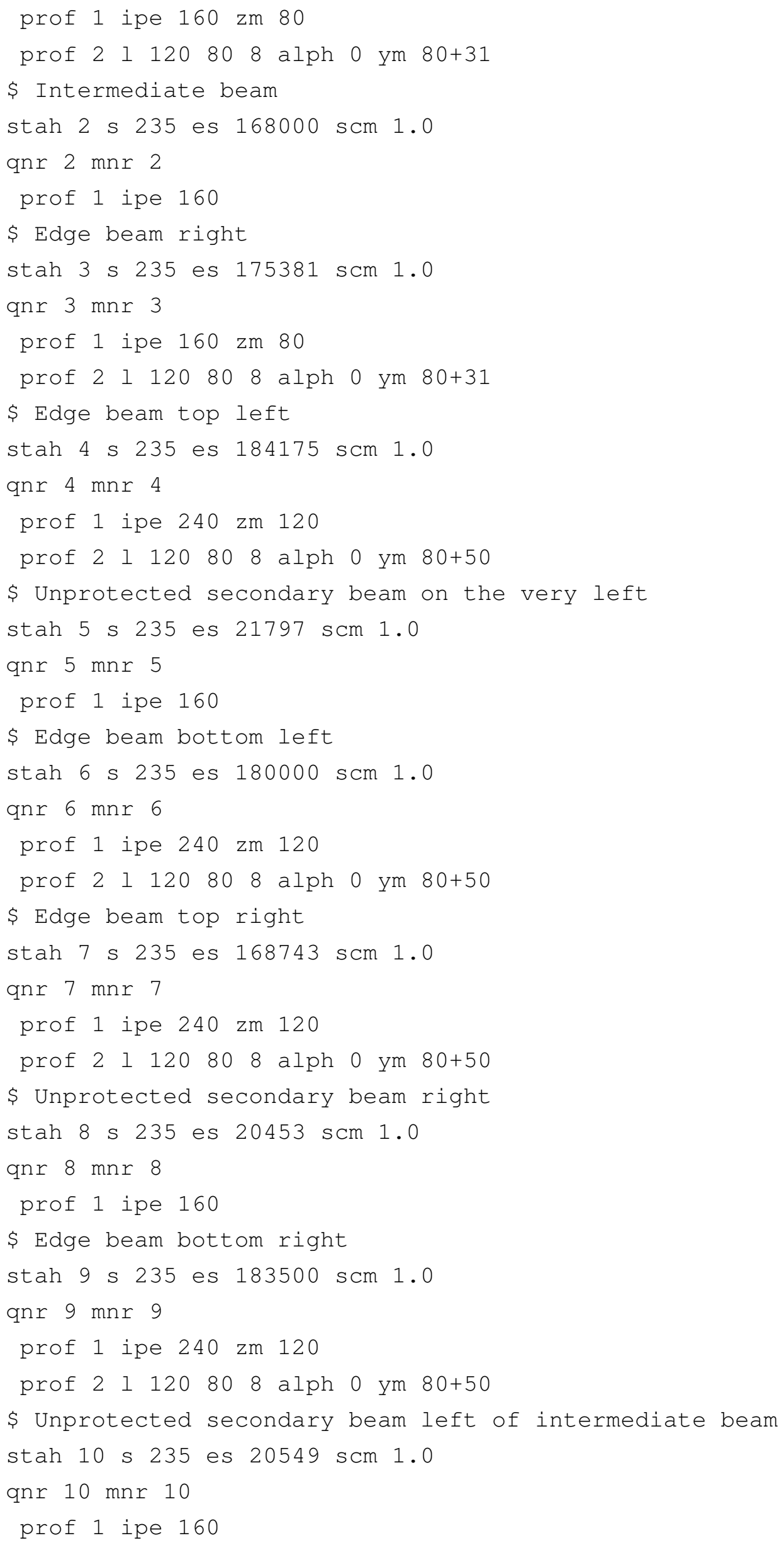


ende

+prog sofimsha

syst raum gdiv 1000

$\$$ Nodes for beams

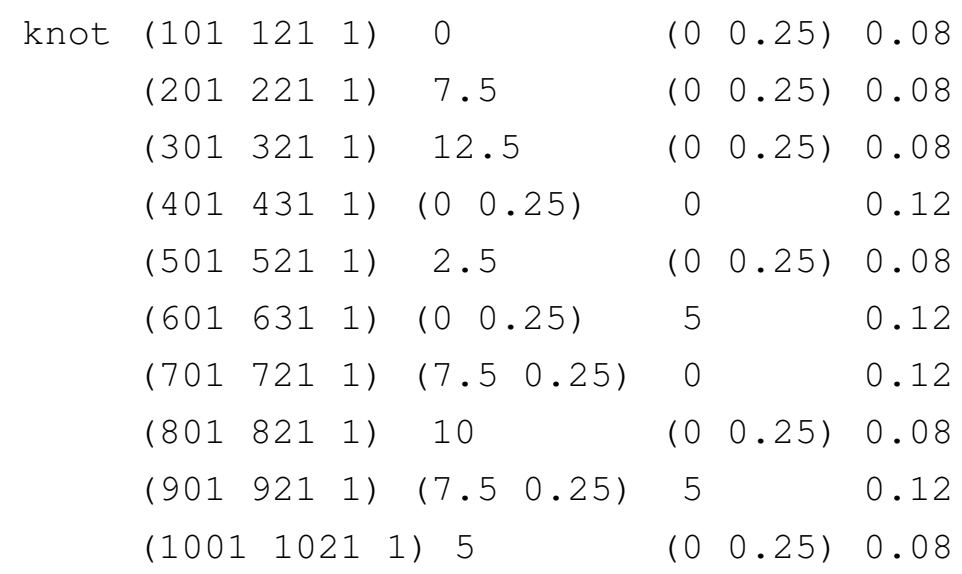

knot 101,121,301,321 fix pz

201 fix pxpz

221 fix pp

\$ Effective slab thickness according to DIN EN 1994-1-2,D.4

let\#h 0.112

\$ Slab depth transversal

let\#hy 0.112

$\$$ Nodes for slab

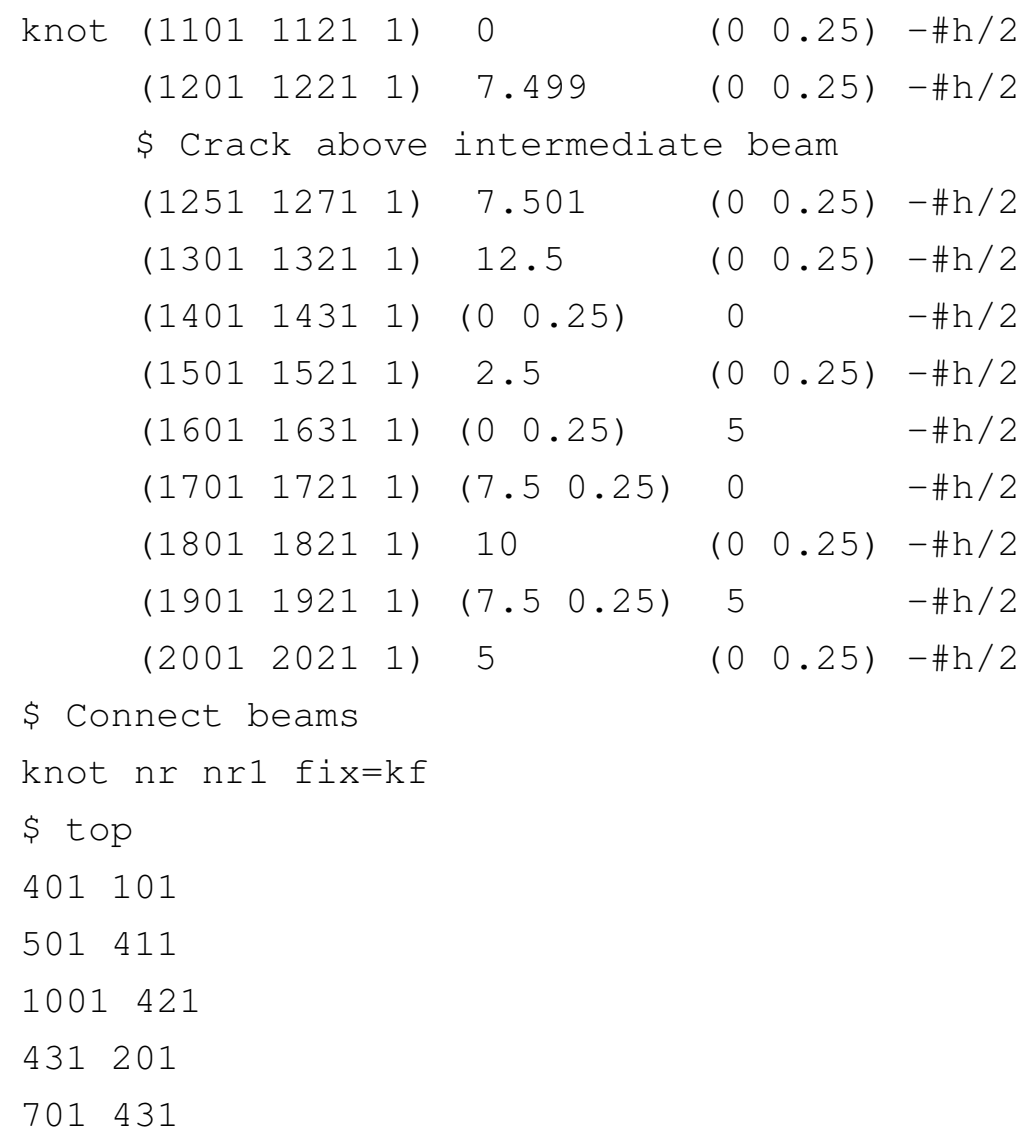


801711

721301

$\$$ bottom

601121

521611

1021621

631221

901631

821911

921321

$\$$ Connect slab to beams

knot nr nrl fix=kf

$\left(\begin{array}{lll}1101 & 1121 & 1\end{array}\right) \quad\left(\begin{array}{ll}101 & 1\end{array}\right)$

$\left(\begin{array}{lll}1301 & 1321 & 1\end{array}\right) \quad(301 \quad 1)$

$\left(\begin{array}{lll}1401 & 1431 & 1\end{array}\right) \quad(401$ 1)

$\left(\begin{array}{lll}1501 & 1521 & 1\end{array}\right) \quad(501 \quad 1)$

$\left(\begin{array}{lll}1601 & 1631 & 1\end{array}\right) \quad(601 \quad 1)$

$\left(\begin{array}{lll}1701 & 1721 & 1\end{array}\right) \quad\left(\begin{array}{ll}701 & 1\end{array}\right)$

$\left(\begin{array}{lll}1801 & 1821 & 1\end{array}\right) \quad(801 \quad 1)$

$\left(\begin{array}{lll}1901 & 1921 & 1\end{array}\right) \quad(901 \quad 1)$

$(20012021$ 1) (1001 1)

$\$$ Connect left slab to intermediate beam

knot $\mathrm{nr}$ nrl fix $=\mathrm{kp}$

$\left(\begin{array}{lll}1202 & 1220 & 1\end{array}\right) \quad(2021)$

fede (1201 1221 1) (1201 1) (201 1) dy $1 \mathrm{~cm}$ lel flie 1.6

knot 1201 fix kf 201

knot 1221 fix $\mathrm{kf} 221$

\$ Connect right slab to intermediate beam

knot nr nrl fix=kp

$\left(\begin{array}{lll}1251 & 1271 & 1\end{array}\right) \quad(1201$ 1)

fede (4251 4271 1) (1251 1) (201 1) dy $1 \mathrm{~cm}$ lel flie 1.6

grup 0 bez Beams

\$ Edge beam left

stab nr ka ke qnr=1 drot=0 $\mathrm{xa}=-0.040 \mathrm{xe}=-0.040 \mathrm{za}=-0.0515 \mathrm{ze}=-0.0515$

101101102 anfa mymzmt

$\left(\begin{array}{lll}102 & 119 & 1\end{array}\right) \quad(1021) \quad(1031)$

120120121 ende mymzmt

\$ Intermediate beam

stab nr ka ke qnr=2

201201202 anfa mymzmt

$\left(\begin{array}{lll}202 & 219 & 1\end{array}\right) \quad(2021) \quad(2031)$

220220221 ende mymzmt

\$ Edge beam right 
stab hr ka ke qnr=3 drot $=0$ xa=0.040 xe=0.040 za=-0.0515 ze=-0.0515

320321320 anfa mymzmt

$\left(\begin{array}{lll}319 & 302 & -1\end{array}\right) \quad(320-1) \quad(319-1)$

301302301 ende mymzmt

\$ Edge beam top left

stab nr ka ke qnr=4 drot=0 ya=-0.032 ye=-0.032 za=-0.0449 ze=-0.0449

430431430 anfa mymzmt

$\left(\begin{array}{lll}429 & 402 & -1\end{array}\right)\left(\begin{array}{lll}430 & -1\end{array}\right)\left(\begin{array}{ll}429 & -1\end{array}\right)$

401402401 ende mymzmt

\$ Unprotected secondary beam on the very left

stab nr ka ke qnr=5

501501502 anfa mymzmt

$\left(\begin{array}{lllll}502 & 519 & 1\end{array}\right) \quad(502 \quad 1) \quad(503 \quad 1)$

520520521 ende mymzmt

\$ Edge beam bottom left

stab nr ka ke qnr=6 drot=0 ya=0.032 ye=0.032 za=-0.0449 ze=-0.0449

601601602 anfa mymzmt

$\left(\begin{array}{lll}602 & 629 & 1\end{array}\right) \quad\left(\begin{array}{lll}602 & 1\end{array}\right)\left(\begin{array}{lll}603 & 1\end{array}\right)$

630630631 ende mymzmt

\$ Edge beam top right

stab nr ka ke qnr=7 drot=0 ya=-0.032 ye=-0.032 za=-0.0449 ze=-0.0449

720721720 anfa mymzmt

$\left(\begin{array}{lll}719 & 702 & -1\end{array}\right) \quad(720-1) \quad(719-1)$

701702701 ende mymzmt

\$ Unprotected secondary beam right

stab nr ka ke qnr=8

801801802 anfa mymzmt

$(8028191) \quad(8021) \quad(803 \quad 1)$

820820821 ende mymzmt

\$ Edge beam bottom right

stab nr ka ke qnr=9 drot=0 ya=0.032 ye=0.032 za=-0.0449 ze=-0.0449

901901902 anfa mymzmt

$(9029191) \quad(902 \quad 1) \quad(9031)$

920920921 ende mymzmt

\$ Unprotected secondary beam left of intermediate beam

stab nr ka ke qur=8

110011002 anfa mymzmt

$\left(\begin{array}{lll}2 & 19 & 1\end{array}\right) \quad(1002$ 1) $(1003$ 1)

2010201021 ende mymzmt

\$ slab

steu gtol 0.001

grup 1 bez 'Larger slab'

quad fit k1 1101112112211201 mnr 20 mbw 21 nra 7 


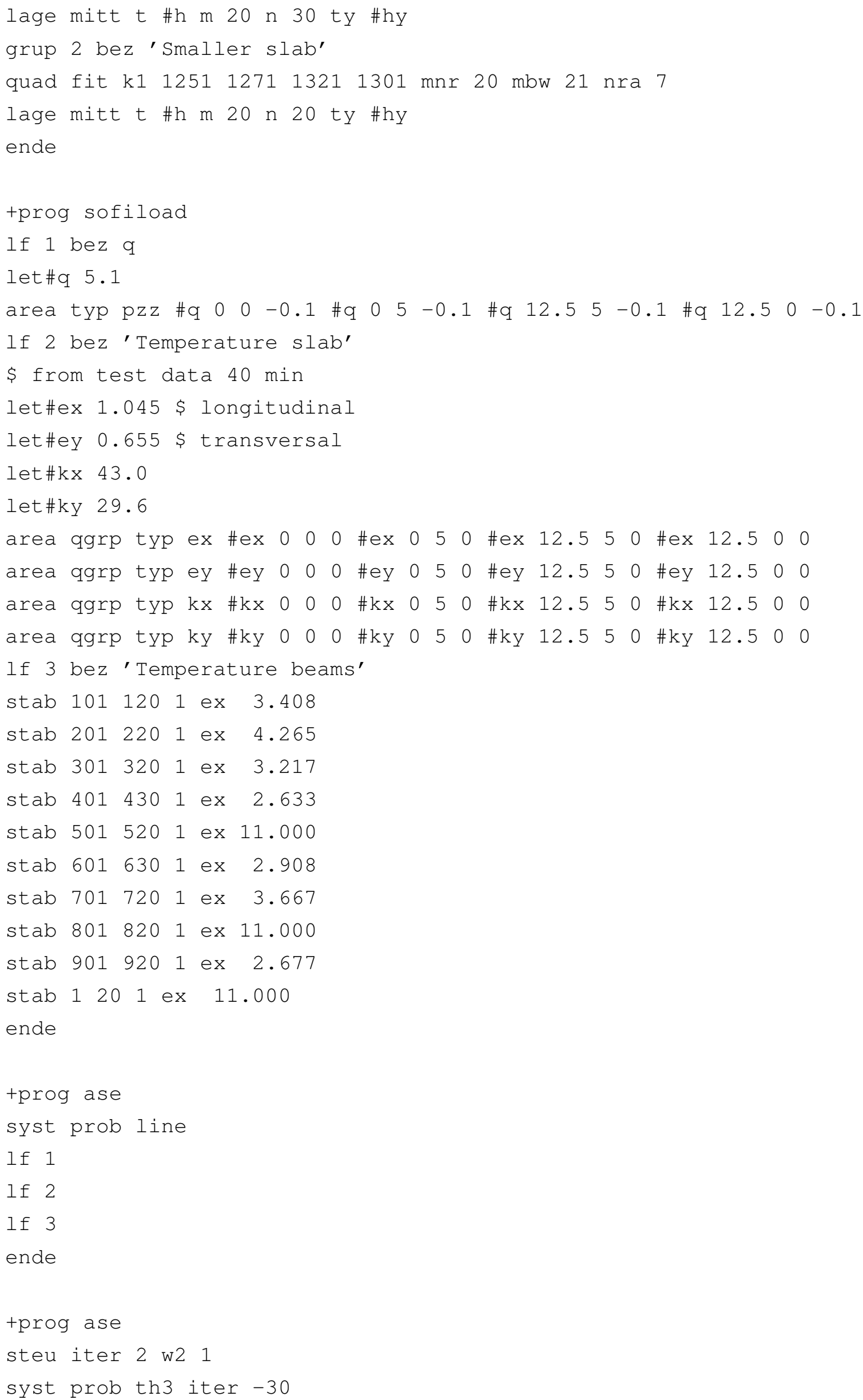




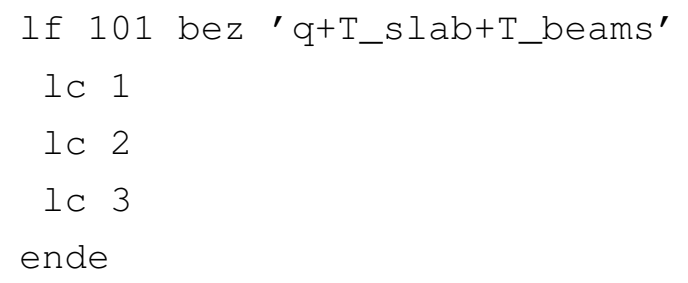

\section{B.8 Validation on FRACOF test}

See Section 7.3.

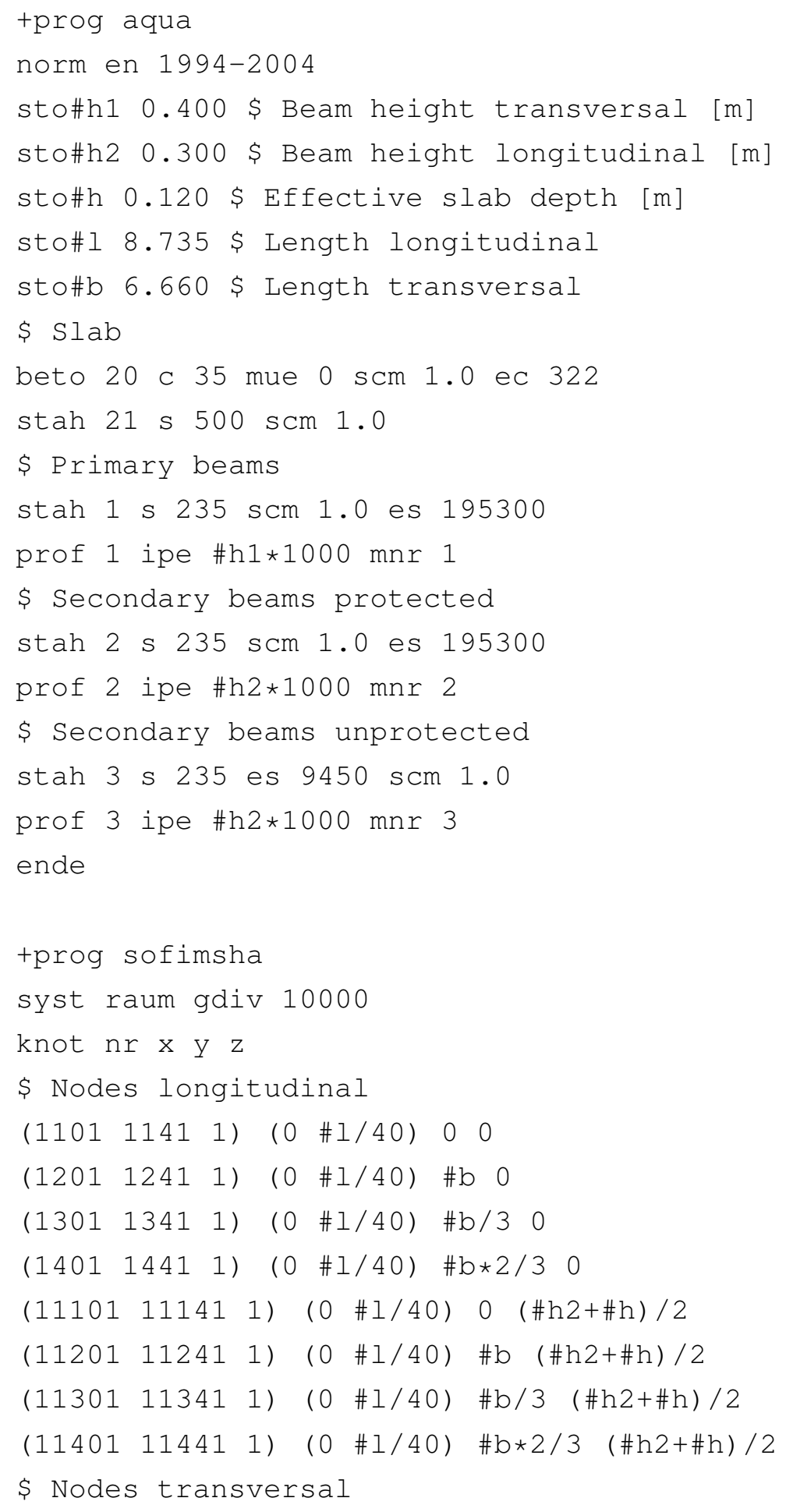




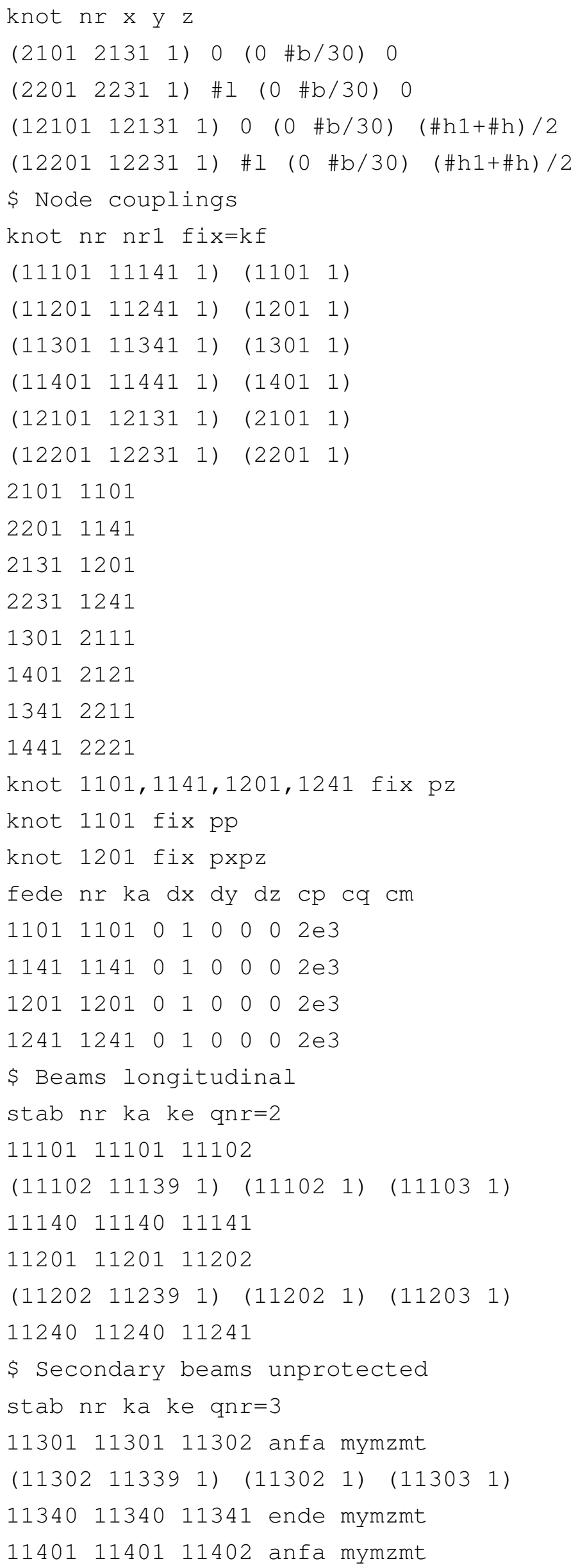




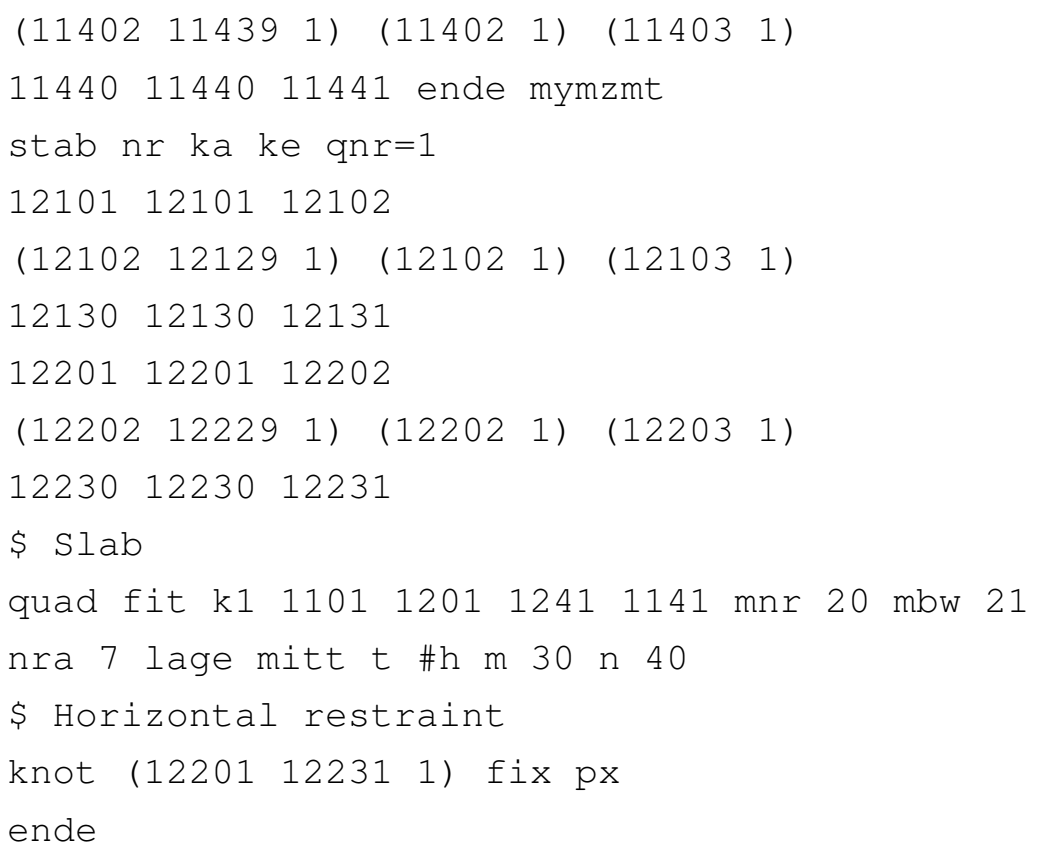




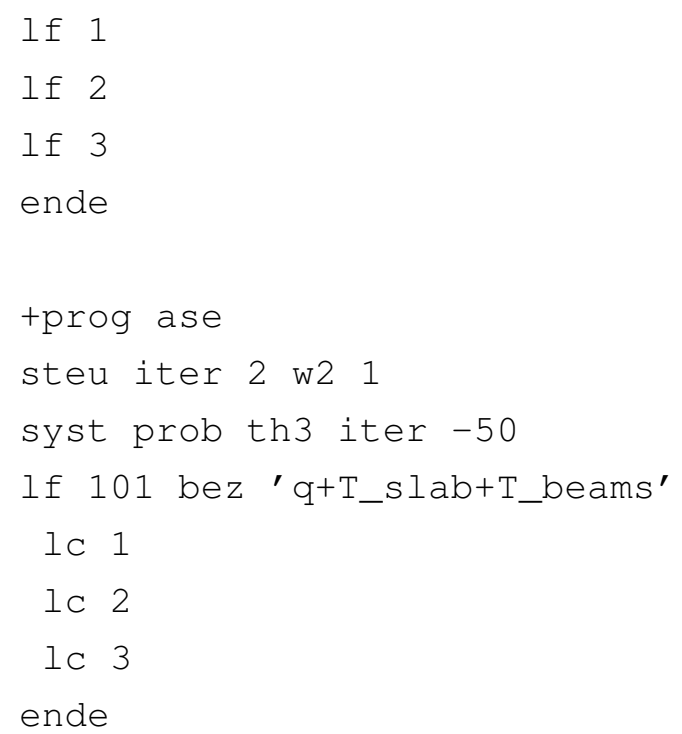

\section{B.9 Worked example}

See Chapter 8.

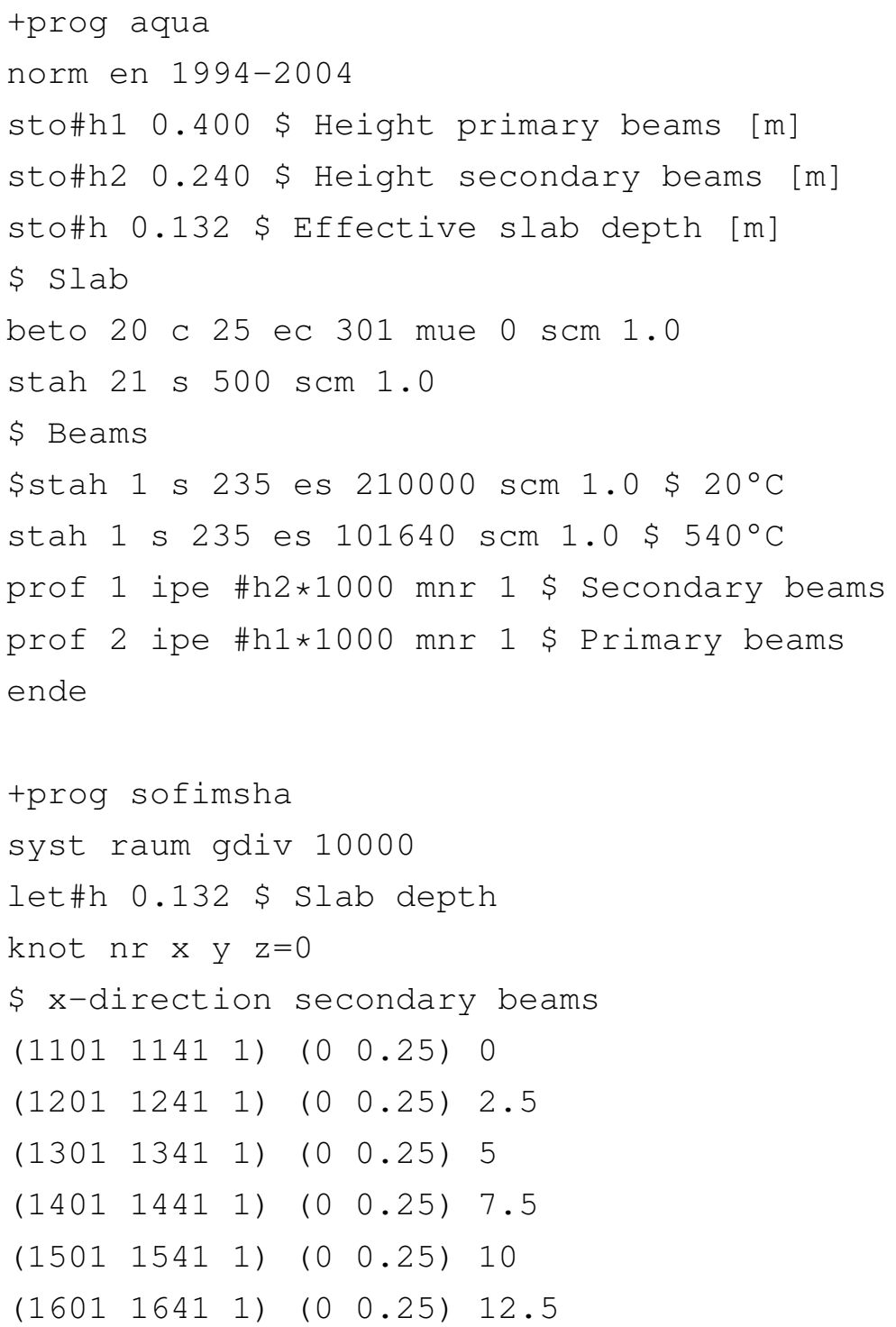




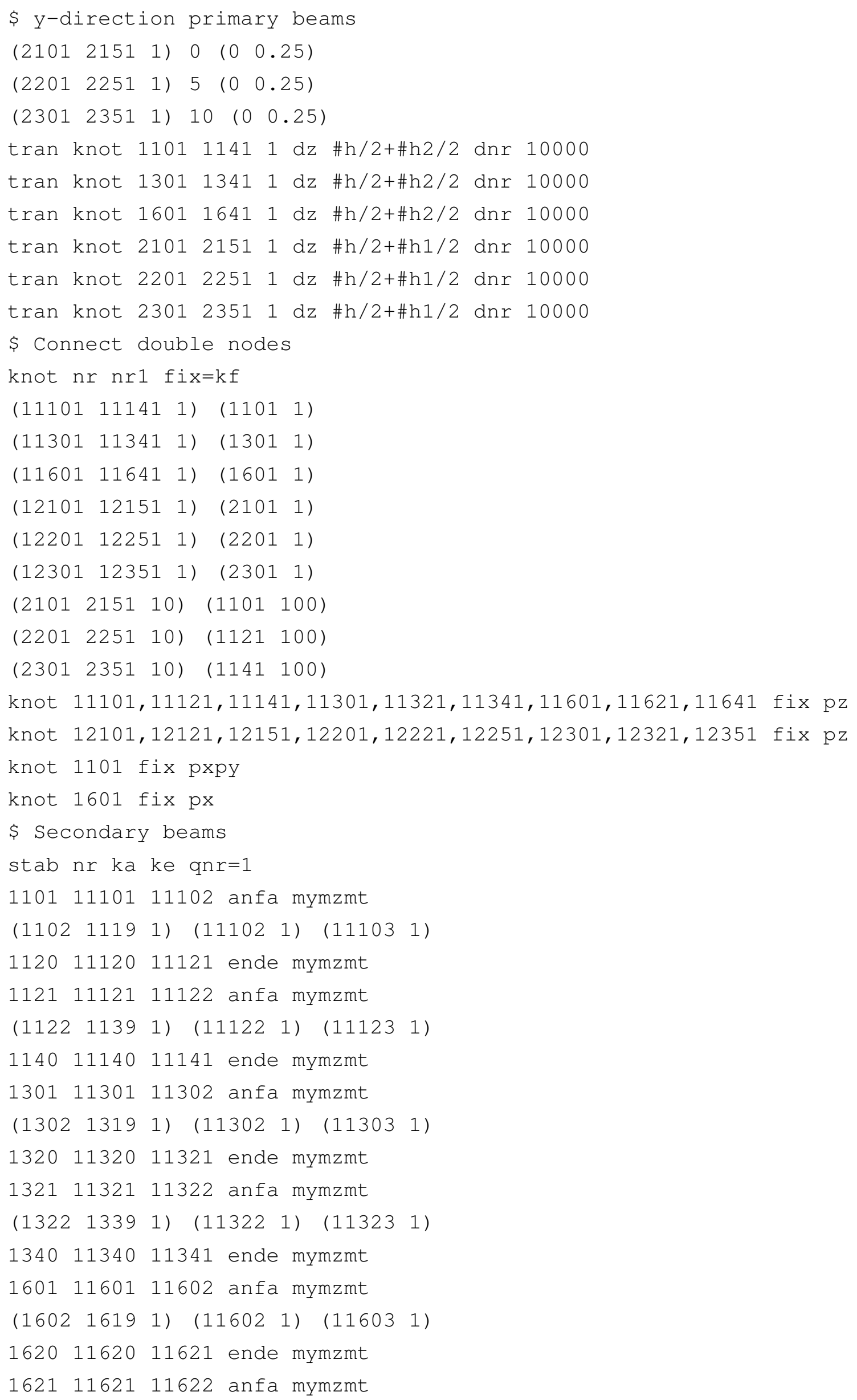




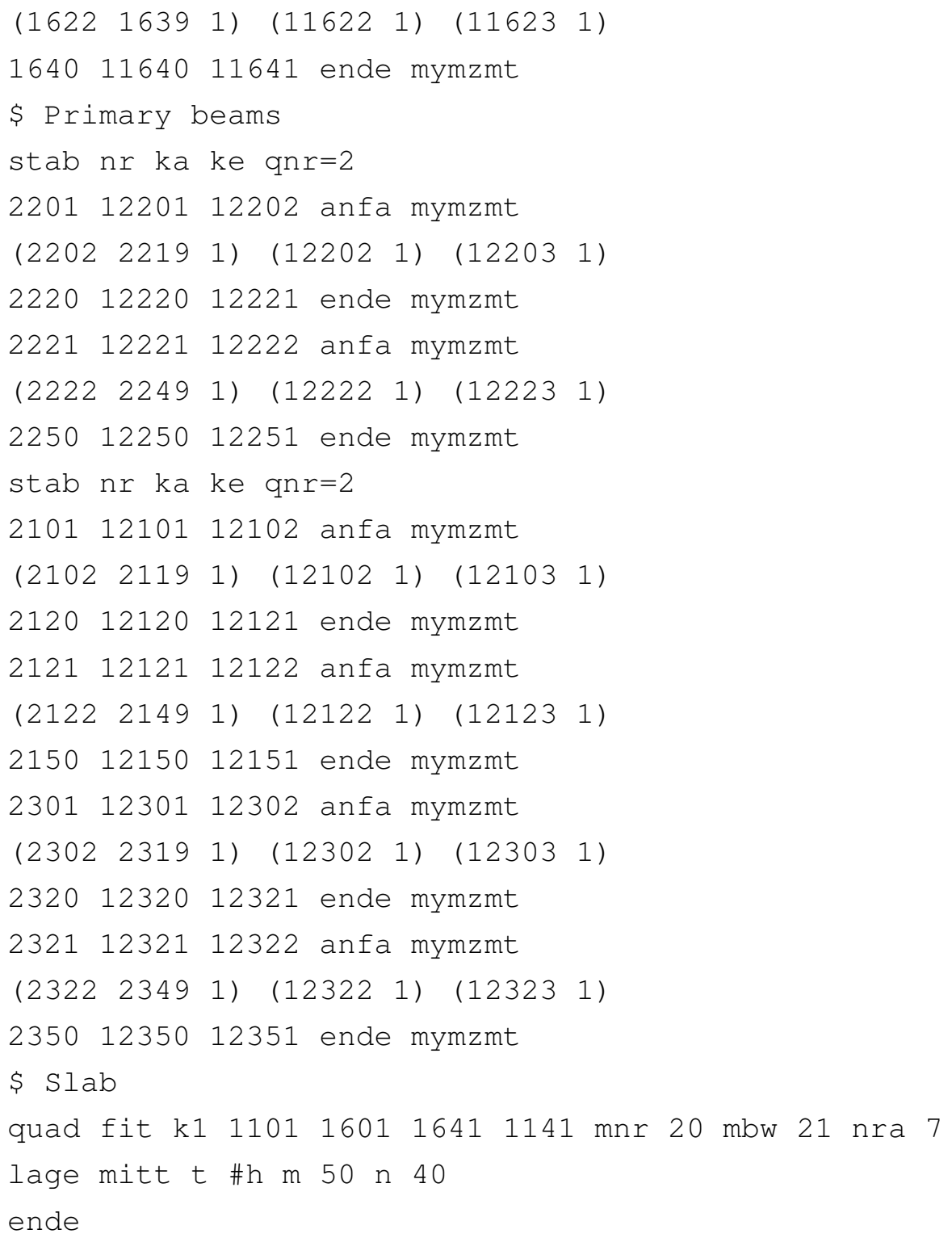




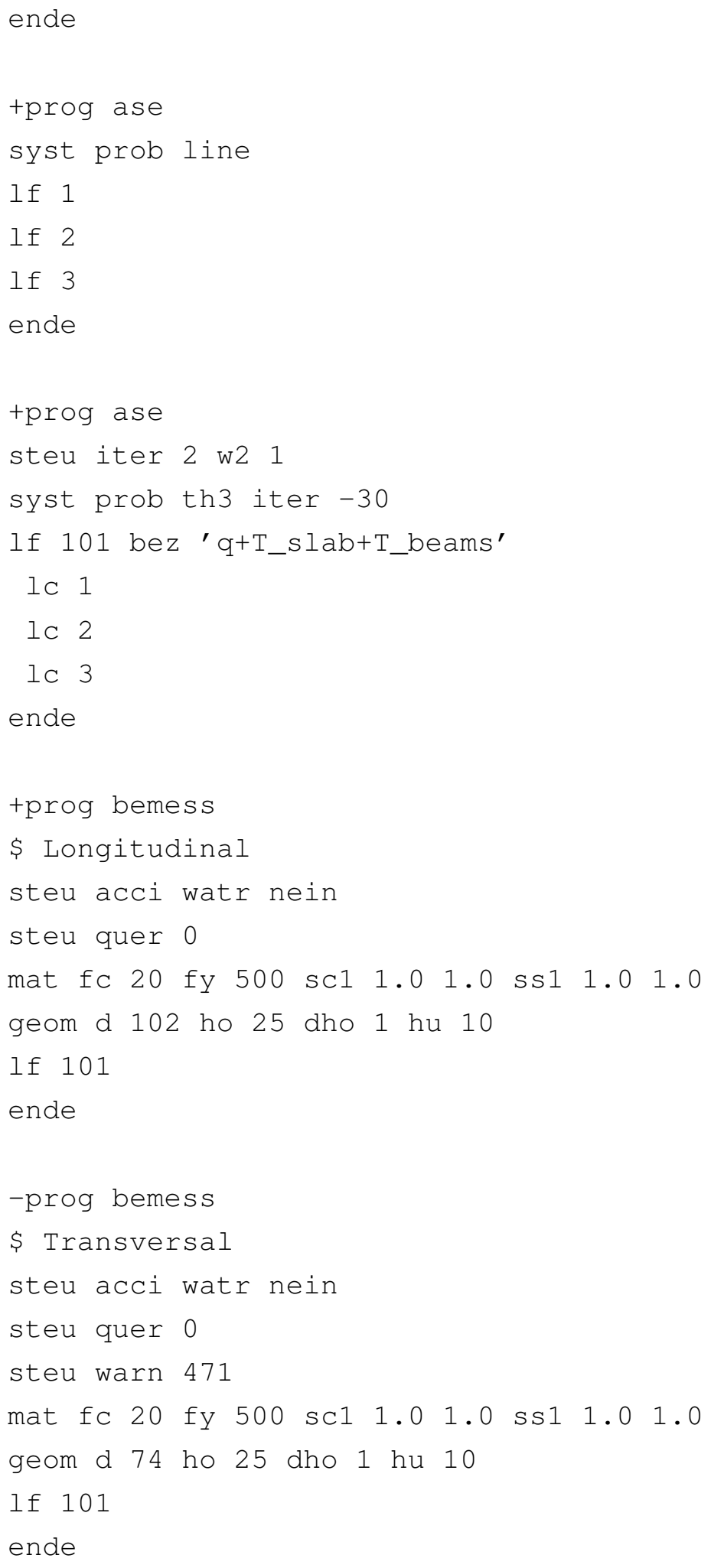




\section{Bibliography}

[1] ABU, A. K.: Behaviour of Composite Floor Systems in Fire, University of Sheffield, Diss., 2009

[2] Abu, A. K. ; Ramanitrarivo, V. ; Burgess, I. W.: Collapse Mechanisms of Composite Slab Panels in Fire. In: Journal of Structural Fire Engineering 2 (2011), Nr. 3, S. 205-216

[3] Altenbach, H. ; Altenbach, J. ; Naumenko, K.: Ebene Flächentragwerke: Grundlagen der Modellierung und Berechnung von Scheiben und Platten. Berlin : Springer, 1998

[4] BAILEY, C.G.: Membrane action of unrestrained lightly reinforced concrete slabs at large displacements. In: Engineering Structures 23 (2001), S. 470-483

[5] BAILEY, C.G.: Efficient arrangement of reinforcement for membrane behaviour of composite floor slabs in fire conditions. In: Journal of Constructional Research 59 (2003), S. 931-949

[6] BAILEY, C.G.: Membrane action of slab / beam composite floor systems in fire. In: Engineering Structures 26 (2004), Nr. 12, S. 1691-1703

[7] BAILeY, C.G. ; Moore, D.B.: The structural behaviour of steel frames with composite floorslabs subject to fire: Part 1: Theory. In: The Structural Engineer 78 (2000), Nr. 11, S. 19-27

[8] Bailey, C.G. ; Moore, D.B.: The structural behaviour of steel frames with composite floorslabs subject to fire: Part 2: Design. In: The Structural Engineer 78 (2000), Nr. 11, S. 28-33

[9] BAILey, C.G. ; ToH, W.S.: Small-scale concrete slab tests at ambient and elevated temperatures. In: Engineering Structures 29 (2007), S. 2775-2791

[10] Both, C.: The Fire Resistance of Composite Steel-Concrete Slabs, TU Delft, Diss., 1998

[11] BRitish STEEL PLC.: The Behaviour of Multi-Storey Steel Framed Buildings in Fire: A European Joint Research Programme. 1999

[12] Building Research Establishment LtD: Results and observations from full-scale fire test at BRE Cardington, 16 January 2003. 2004

[13] CAdorin, J.-F. ; Franssen, J.-M.: A tool to design steel elements submitted to compartment fires - OZone V2. Part 1: pre- and post-flashover compartment fire model. In: Fire Safety Journal 38 (2003), S. 395-427

[14] Cadorin, J.-F. ; Pintea, D. ; Dotreppe, J.-C. ; Franssen, J.-M.: A tool to design steel elements submitted to compartment fires - OZone V2. Part 2: Methodology and application. In: Fire Safety Journal 38 (2003), S. 429-451 
[15] Cameron, N. J. K.: The Behaviour and Design of Composite Floor Systems in Fire, University of Edinburgh, Diss., 2003

[16] Cameron, N. J. K. ; Usmani, A. S.: New design method to determine the membrane capacity of laterally restrained composite floor slabs in fire Part 1: Theory and method. In: The Structural Engineer 83 (2005), Nr. 19

[17] Cameron, N. J. K. ; Usmani, A. S.: New design method to determine the membrane capacity of laterally restrained composite floor slabs in fire Part 2: Validation. In: The Structural Engineer 83 (2005), Nr. 19

[18] DIN 18230-3: Baulicher Brandschutz im Industriebau - Teil 3: Rechenwerte. August 2002

[19] DIN 4102-4: Brandverhalten von Baustoffen und Bauteilen - Zusammenstellung und Anwendung klassifizierter Baustoffe, Bauteile und Sonderbauteile. March 1994

[20] DIN EN 13381-8: Prüfverfahren zur Bestimmung des Beitrages zum Feuerwiderstand von tragenden Bauteilen - Teil 8: Reaktive Ummantelung von Stahlbauteilen. September 2010

[21] DIN EN 1990: Eurocode: Grundlagen der Tragwerksplanung. December 2010

[22] DIN EN 1990/NA: Nationaler Anhang - National festgelegte Parameter - Eurocode: Grundlagen der Tragwerksplanung. December 2010

[23] DIN EN 1991-1-1: Eurocode 1: Einwirkungen auf Tragwerke - Teil 1-1: Allgemeine Einwirkungen auf Tragwerke - Wichten, Eigengewicht und Nutzlasten im Hochbau. December 2010

[24] DIN EN 1991-1-2: Eurocode 1: Einwirkungen auf Tragwerke - Teil 1-2: Allgemeine Einwirkungen - Brandeinwirkungen auf Tragwerke. December 2010

[25] DIN EN 1991-1-2/NA: Nationaler Anhang - National festgelegte Parameter - Eurocode 1: Einwirkungen auf Tragwerke - Teil 1-2: Allgemeine Einwirkungen - Brandeinwirkungen auf Tragwerke. December 2010

[26] DIN EN 1992-1-1: Eurocode 2: Bemessung und Konstruktion von Stahlbeton- und Spannbetontragwerken - Teil 1-1: Allgemeine Bemessungsregeln und Regeln für den Hochbau. January 2011

[27] DIN EN 1992-1-2: Eurocode 2: Bemessung und Konstruktion von Stahlbeton- und Spannbetontragwerken - Teil 1-2: Allgemeine Regeln - Tragwerksbemessung für den Brandfall. December 2010

[28] DIN EN 1992-1-2/NA: Nationaler Anhang - National festgelegte Parameter - Eurocode 2: Bemessung und Konstruktion von Stahlbeton- und Spannbetontragwerken - Teil 1-2: Allgemeine Regeln - Tragwerksbemessung für den Brandfall. December 2010

[29] DIN EN 1993-1-2: Eurocode 3: Bemessung und Konstruktion von Stahlbauten - Teil 1-2: Allgemeine Regeln - Tragwerksbemessung für den Brandfall. December 2010 
[30] DIN EN 1993-1-2/NA: Nationaler Anhang - National festgelegte Parameter - Eurocode 3: Bemessung und Konstruktion von Stahlbauten - Teil 1-2: Allgemeine Regeln - Tragwerksbemessung für den Brandfall. December 2010

[31] DIN EN 1994-1-1: Eurocode 4: Bemessung und Konstruktion von Verbundtragwerken aus Stahl und Beton - Teil 1-1: Allgemeine Bemessungsregeln und Anwendungsregeln für den Hochbau. December 2010

[32] DIN EN 1994-1-2: Eurocode 4: Bemessung und Konstruktion von Verbundtragwerken aus Stahl und Beton - Teil 1-2: Allgemeine Regeln - Tragwerksbemessung für den Brandfall. December 2010

[33] DIN EN 1994-1-2/NA: Nationaler Anhang - National festgelegte Parameter - Eurocode 4: Bemessung und Konstruktion von Verbundtragwerken aus Stahl und Beton - Teil 1-2: Allgemeine Regeln - Tragwerksbemessung für den Brandfall. December 2010

[34] DIN-FACHBERICHT 102: Betonbrücken. March 2009

[35] Foster, S.J. ; BAiley, C.G. ; Burgess, I.W. ; Plank, R.J.: Experimental behaviour of concrete floor slabs at large displacements. In: Engineering Structures 26 (2004), Nr. 9, S. 1231-1247

[36] Franssen, J. M. ; Kodur, V. K. R. ; Mason, J.: Elements of Theory for Safir 2002: A Computer Program for Analysis of Structures Submitted to the Fire. University of Liege, Department Mécanique des matériaux \& Structures, 2002

[37] Franssen, J.-M. ; Zahira, R.: Design of Steel Structures subjected to Fire. Liège, 2006

[38] Hertz, K.: Simplified calculation method for fire exposed concrete structures: Supporting document for CEN PR-ENV 1992-1-2. Background documents to EN 1992-1-2, Eurocode 2: Design of concrete structures - Part 1-2: General rules - Structural fire design, Amended 5 July 2004, 1993

[39] Hertz, K.: Analyses of Concrete Structures Exposed to Fire: Part 2. Structures. Technical University of Denmark, 2007 (Lecture notes)

[40] Huang, Z. ; Burgess, I. W. ; Plank, R. J.: Modeling Membrane Action of Concrete Slabs in Composite Buildings in Fire. I: Theoretical Development. In: Journal of Structural Engineering 129 (2003), Nr. 8, S. 1093-1102

[41] Huang, Z. ; Burgess, I. W. ; Plank, R. J.: Modeling Membrane Action of Concrete Slabs in Composite Buildings in Fire. II: Validations. In: Journal of Structural Engineering 129 (2003), Nr. 8, S. 1103-1112

[42] Mensinger, M. ; Schaumann, P. ; Stadler, M. ; Sothmann, J.: Nutzung der Membranwirkung von Verbundträger-Decken-Systemen im Brandfall. DASt-Forschungsbericht 2012 (forthcoming)

[43] Mensinger, M. ; Schaumann, P. ; Stadler, M. ; Sothmann, J.: Membranwirkung von Verbunddecken bei Brand - Stand der Technik. In: Stahlbau 79 (2010), Nr. 4, S. 298-305

[44] Newman, G. M. ; Robinson, J. T. ; Bailey, C. G.: Fire safe design: A new approach to multi-storey steel-framed buildings. Ascot : Steel Construction Institute, 2000 (SCI publication P288) 
[45] PolifKe, W. ; Kopitz, J.: Wärmeübertragung: Grundlagen, analytische und numerische Methoden. München : Pearson Studium, 2009

[46] SCHNEIDER, U.: Grundlagen der Ingenieurmethoden im Brandschutz. Düsseldorf : Werner Verlag, 2002

[47] Siegmund, O.: Implementierung der X-FEM in ANSYS. Fachhochschule Hannover, Fachbereich Maschinenbau, Diplomarbeit, 2008

[48] Simms, W. I. ; Zhao, B.: Fire Resistance Assessment of Partially Protected Composite Floors (FRACOF) Design Guide. The Steel Construction Institute (SCI) \& Centre Technique Industriel de la Construction Métallique (CTICM), 2009

[49] Simms, W. I. ; ZhaO, B.: Fire Resistance Assessment of Partially Protected Composite Floors (FRACOF): Engineering Background. The Steel Construction Institute (SCI) \& Centre Technique Industriel de la Construction Métallique (CTICM), 2009

[50] Stadler, M. ; Mensinger, M. ; Schaumann, P. ; Sothmann, J.: Munich Fire Tests on Membrane Action of Composite Slabs in Fire: Test Results and Recent Findings. In: Proceedings of International Conference, Applications of Structural Fire Engineering. Prag, 2011, S. 177-182

[51] TeSAR, C. N.: Zum Tragverhalten von Verbunddeckensystemen im Brandfall, ETH Zürich, Diss., 2008

[52] Vassart, O. ; Bailey, C. G. ; Hawes, M. ; Nadjai, A. ; Simms, W. I. ; ZhaO, B. ; Gernay, T. ; Franssen, J.-M.: Large-Scale Fire Test of Unprotected Cellular Beam Acting in Membrane Action. In: Structures in Fire: Proceedings of the Sixth International Conference. Michigan State University, 2010, S. 398-406

[53] WAld, F. ; JÁNA, T. ; HoROVÁ, K.: Design of joints to composite columns for improved fire robustness: To demonstration fire tests. Czech Technical University in Prague, 2011

[54] Wald, F. ; Kallerová, P. ; Chlouba, P. ; Sokol, Z. ; Strejcek, M. ; Pospisil, J. ; Stroner, M. ; Kremen, T. ; Smitka, V.: Fire Test on an Administrative Building in Mokrsko: Summary of the Results from the Fire Experiment. Prag : Czech Technical University, 2010

[55] Zilch, K. ; Zehetmaier, G.: Bemessung im konstruktiven Betonbau: Nach DIN 1045-1 (Fassung 2008) und EN 1992-1-1 (Eurocode 2). Springer Verlag, 2010 